\title{
ESTIMATION OF SEA SURFACE WAVE SPECTRA USING ACOUSTIC TOMOGRAPHY
}

\author{
by
}

\author{
James Henry Miller
}

B.S. Electrical Engineering, Worcester Polytechnic Institute, 1979

M.S. Electrical Engineering, Stanford University, 1981

submitted in partial fulfillment of

the requirements for the degree of

DOCTOR OF SCIENCE

at the

MASSACHUSETTS INSTITUTE OF TECHNOLOGY

and the

WOODS HOLE OCEANOGRAPHIC INSTITUTION

August, 1987

(C) James Henry Miller 1987

The author hereby grants to MIT permission to reproduce and distribute copies of this thesis in whole or in part.

Signature of Author

Department of Ocean Engineering, MIT and the

MIT-WHOI Joint Program in Oceanographic Engineering

Certified by

Dr. James F Lynch

Thesis Supervisor

Accepted by

Dr. George V. Frisk

Chairman, Joint Committee for Oceanographic Engineering, Massachusetts Institute of Technology-Woods Hole Oceanographic Institution 
ESTIMATION OF SEA SURFACE WAVE SPECTRA USING ACOUSTIC TOMOGRAPHY

by

\author{
James Henry Miller \\ Submitted in partial fulfillment of the requirements for the degree of \\ Doctor of Science at the Massachusetts Institute of Technology \\ and the Woods Hole Oceanographic Institution
}

August 3, 1987

\begin{abstract}
This thesis develops a new technique for estimating quasi-homogeneous and quasi-stationary sea surface wave frequency-direction spectra using acoustic tomography. The analysis of acoustic (mode and ray) phase and travel time perturbations due to a rough sea surface is presented. Two canonical waveguides (ideal shallow water and linear squared index of refraction) are used as examples for the mode perturbation. The analysis is used to explain high mode coherence measured in the FRAM IV experiment. The forward problem of computing the acoustic phase and travel time perturbation spectra given the surface wave spectrum is solved to first order. An application of the technique to ray phase data taken during the MIZEX ' 84 experiment is shown. The inverse problems for the homogeneous and quasi-homogeneous frequency-direction spectrum are introduced. The theory is applied to synthetic data which simulate a fetch-dependent sea. The estimates made agree well with the "actual" (synthetic data) spectrum. The effect of noise in the travel time estimates is studied. The sensitivity of the technique to the number of rays used in the inversion is investigated and the resolution and variance of the inverse method are addressed.
\end{abstract}

Thesis Supervisor:

Dr. James F. Lynch, Associate Scientist

Woods Hole Oceanographic Institution 


\section{Acknowledgements}

I would like to thank my thesis advisor, Dr. James F. Lynch, for his guidance, inspiration, and support. He provided motivation when I needed it, freedom when I was ready to use it, and friendship through it all.

I am also grateful to my thesis committee for their efforts: Arthur B. Baggeroer, Eugene A. Terray, Michael J. Buckingham, Robert C. Spindel, and ChingSang Chiu. Ching-Sang helped me for four years, first as fellow student, then as post-doc, and now as scientist. Thanks also to Sandy Williams for serving as the chairman of the defense, to Greg Duckworth, now at BBN, for reviewing my thesis proposal, and to Yves Desaubies, now at IFREMER, for his advice early on.

I especially thank Hans Graber for discussions on surface waves, James Riley and Subramanium Rajan on inverse theory, Josko Catapovic on array theory, and Kevin Casey on asymptotic analysis. For technical discussions, I thank George Frisk, Jules Jaffe, Harry Jenter, John Wilkin, Ferd Diemer, Paul Bushong, Andy Trivett, and Peter Dahl. A special thanks goes to Mike Wengrovitz, now at Atlantic Aerospace Electronics, for his advice and encouragement. I received much help on computers from Art Newhall, Chris Dunn, Jim Doutt, and Chris Lynch. I also thank Betsy Hirschel for help with the artwork, and Stanley Rosenblad for technical support. I gratefully acknowledge the financial support of the Office of Naval Research, the General Electric Foundation, and the Ford Foundation.

Finally, I thank my wife, Linda, for her love and patience through it all. Her advice, from the very beginning, greatly contributed to this work. Thanks to my daughters, Mary Beth and Eileen, for being patient with their dad. I am also grateful to Linda's parents, Mr. and Mrs. John H. Murray, and her whole family, for their emotional and financial support. 
To Linda, Mary Beth, and Eileen. 


\section{Contents}

Abstract 2

Acknowledgements $\quad 3$

List of Symbols $\quad 14$

1 Introduction $\quad 20$

1.1 Background ...................... 20

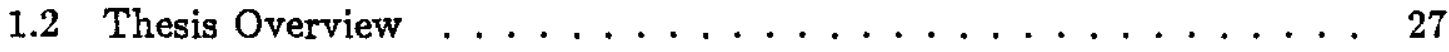

2 Acoustic Scattering by a Rough Sea Surface 29

2.1 Normal Modes ...................... 30

2.1.1 Normal Modes in a Stratified Ocean ............ 31

2.1.2 Normal Modes in a Range Dependent Ocean . . . . . . . 33

2.1.3 Phase and Travel Time Variations ............ 37

2.1.4 Canonical Waveguide Fluctuation Parameters . . . . . . . 41

2.1.5 Cross-Coherence of Modes in the Arctic . . . . . . . . . 51

2.1.6 Coupled Mode Effects . . . . . . . . . . . . 55

2.1.7 Coupled Mode Bragg Scattering . . . . . . . . . . . . 59

2.1.8 Coupling Strength ................. 73

2.1.9 WKB Approximation Breakdown . . . . . . . . . . 75 
2.2 Rays . . . . . . . . . . . . . . . . . 76

2.2.1 Eikonal and Transport Equations . . . . . . . . . . 77

2.2.2 Scattering of Rays from a Rough Surface . . . . . . . 78

2.3 Summary of Scattering Results . . . . . . . . . . . . . 79

3 Surface Wave Spectra - The Forward Problem 81

3.1 Surface Wave Spectra Review . . . . . . . . . . . . . 82

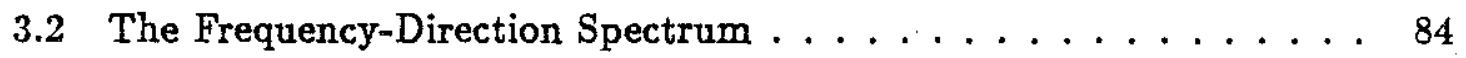

3.2.1 Spectrum Features . . . . . . . . . . . . 85

3.2.2 A Model Spectrum . . . . . . . . . . . . . . 86

3.3 Mode Phase and Travel Time Spectra $\ldots \ldots \ldots \ldots \ldots$. . . 87

3.4 Ray Perturbation Spectra . . . . . . . . . . . . . . 92

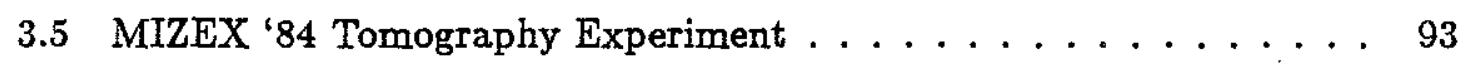

3.6 The Frozen Approximation $\ldots \ldots \ldots \ldots \ldots \ldots \ldots$

4 Wave Spectra Estimation with Tomography 104

4.1 Inversion of Modes . . . . . . . . . . . 105

4.1.1 Posing the mode inverse problem . . . . . . . . . 106

4.1.2 Model Discretization . . . . . . . . . . . . . . . 109

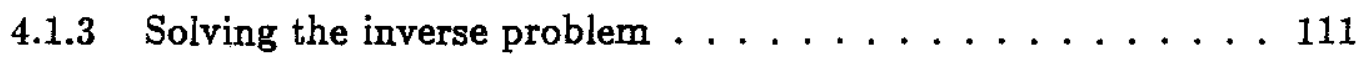

4.1.4 Smoothing Matrices . . . . . . . . . . . . . 113

4.1.5 Resolution and Variance Tradeoff . . . . . . . . . . . 114

4.1.6 Mode Inversion Results: No Noise Case . . . . . . . . 116

4.2 Inversion of Rays . . . . . . . . . . . . . . . 124

4.2.1 Posing the Ray Inverse Problem . . . . . . . . . . 125

4.2.2 Six Ray Inversion Results: No Noise Case . . . . . . . 126

4.2.3 Six Ray Inversion Results: Noisy Data . . . . . . . . . 126

4.2.4 Six Ray Inversion Results: Very Noisy Data . . . . . . . . 139 
4.2.5 Integration with Directional Wave Buoy . . . . . . . . 139

4.2.6 Three Ray Inversion: Noisy Data . . . . . . . . . . . 145

4.2.7 Nine Ray Inversion: Noisy Data . . . . . . . . . . 145

4.3 Resolution and Variance ................... 150

5 Conclusions $\quad 158$

5.1 Summary . . . . . . . . . . . . . . . . . 158

5.2 Comparison with Other Methods . . . . . . . . . . . . 160

5.3 Original Contributions ..................... 161

5.4 Significance of the Thesis . . . . . . . . . . . . 162

5.5 Future Directions . . . . . . . . . . . . . . 163

Biography

172 


\section{List of Figures}

2.1 Horizontally stratified waveguide. . . . . . . . . . . . 31

2.2 Adiabatic mode adapting to changes in sea surface height. . . . . . . 38

2.3 Travel time wander and bias due to surface waves. . . . . . . . 40

2.4 Ideal hard bottom shallow water waveguide . . . . . . . . . . . 42

2.5 FRAM IV sound speed profile and bi-linear approximation . . . . 54

2.6 Schematic of coupled mode scattering . . . . . . . . . 59

2.7 Coupled mode pressure amplitude for resonant Bragg scattering. . . 62

2.8 Ray reflected from rough surface . . . . . . . . . . . . 79

3.1 Typical frequency-direction spectrum. . . . . . . . . . . 87

3.2 A realization of a sea surface from a typical frequency-direction spectrum. ......................... 88

3.3 Typical variation in directional dependence and kernal. . . . . . . . . 90

3.4 Geometry for the MIZEX '84 tomography experiment . . . . . . . 9 95

3.5 Sound velocity profile at the WHOI tomography source. . . . . . . . 96

3.6 Sound velocity profile near the NUSC reciever. . . . . . . . . . . 97

3.7 Range dependent ray trace between the WHOI source and the NUSC receiver. . . . . . . . . . . . . . . . . . 98

3.8 Phase of the prominent arrival at NUSC receiver on day 168 with mean subtracted out. . . . . . . . . . . . . . . 99 
3.9 Spectrum of the phase of prominent arrival at NUSC receiver on day $168 \ldots \ldots \ldots \ldots$. . . . . . . . . . . . 100

3.10 Phase of the prominent arrival at NUSC receiver on day 169 with mean subtracted out. . . . . . . . . . . . . 100

3.11 Spectrum of the phase of prominent arrival at NUSC receiver on day $169 . \ldots \ldots \ldots$. . . . . . . . . . . . . . 101

3.12 Surface wave spectra obtained for days 168 and 169 during MIZEX' 84 . 101

3.13 Frequency-dependent bearing error due to the frozen approximation. 103

4.1 Geometry for discretizing the relation between travel time spectra and surface wave frequency-direction spectra. . . . . . . . 107

4.2 Frequency-direction spectrum angular and spatial discretization. . . 110

4.3 Tomographic array and acoustic mode paths. . . . . . . 110

4.4 Tomographic array location. . . . . . . . . . . . . . 111

4.5 Graphical representation of the kernel G. . . . . . . . . 112

4.6 Tradeoff curve between resolution and variance. . . . . . . . . 116

4.7 Contour plot grey level key. . . . . . . . . . . . . . 117

4.8 Contour plot of inversions of modes with no noise for boxes 1,4 , and

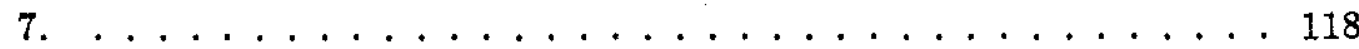

4.9 Contour plot of inversions of modes with no noise for boxes 2,5 , and 8. . . . . . . . . . . . . . . . . . . . . . 119

4.10 Contour plot of inversions of modes with no noise for boxes 3,6 , and $9 . \ldots \ldots \ldots \ldots \ldots \ldots \ldots \ldots \ldots \ldots \ldots$

4.11 Inversion of modes with no noise versus frequency at direction of 90 deg. . . . . . . . . . . . . . . . . . 122 
4.12 Inversion of modes with no noise versus direction at a frequency of $0.2 \mathrm{~Hz} \ldots \ldots \ldots \ldots \ldots \ldots$

4.13 Ocean waveguide for inversions. . . . . . . . . . . . . 124

4.14 Contour plot of inversions of six rays with no noise for boxes 1,4 ,

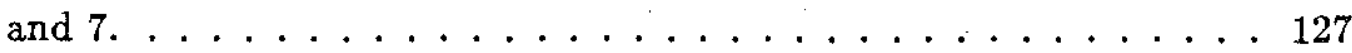

4.15 Contour plot of inversions of six rays with no noise for boxes 2,5 , and $8 . \ldots \ldots \ldots \ldots \ldots \ldots \ldots$

4.16 Contour plot of inversions of six rays with no noise for boxes 3,6 ,

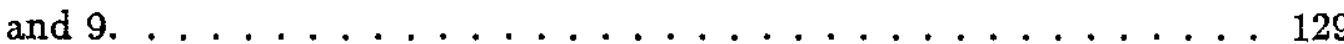

4.17 Inversion of six rays with no noise versus frequency at direction of 90

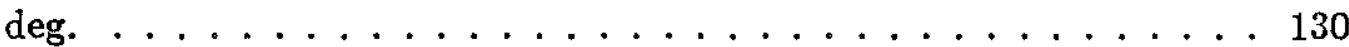

4.18 Inversion of six rays with no noise versus direction at a frequency of

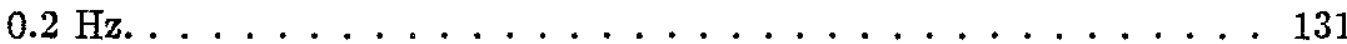

4.19 Contour plot of inversions of six rays with noise for boxes 1,4 , and 7.134

4.20 Contour plot of inversions of six rays with noise for boxes 2,5 , and 8.135

4.21 Contour plot of inversions of six rays with noise for boxes 3,6 , and 9.136

4.22 Inversion of six rays with noise versus frequency at direction of $90 \mathrm{deg} .137$

4.23 Inversion of six rays with noise versus direction at a frequency of 0.2 $\mathrm{Hz} \ldots \ldots \ldots \ldots \ldots \ldots$

4.24 Contour plot of inversions of six rays with more noise for boxes 1,4 , and $7 \ldots \ldots \ldots \ldots \ldots \ldots \ldots$

4.25 Contour plot of inversions of six rays with more noise for boxes 2,5 , and $8 \ldots \ldots \ldots \ldots \ldots \ldots \ldots \ldots \ldots \ldots$

4.26 Contour plot of inversions of six rays with more noise for boxes 3,6 ,

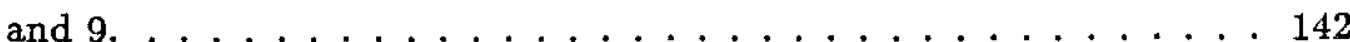

4.27 Inversion of six rays with more noise versus frequency at direction of

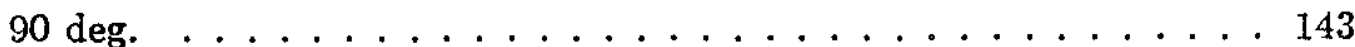


4.28 Inversion of six rays with more noise versus direction at a frequency

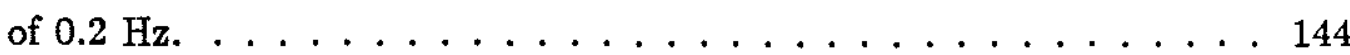

4.29 Inversion of six rays and directional wave buoy versus frequency at direction of $90 \mathrm{deg} \ldots \ldots \ldots \ldots \ldots \ldots$

4.30 Inversion of six rays and directional wave buoy versus direction at a frequency of $0.2 \mathrm{~Hz} . \ldots \ldots \ldots \ldots \ldots \ldots \ldots$

4.31 Inversion of three rays with noise versus frequency at direction of 90 deg. . . . . . . . . . . . . . . . . . . . 148

4.32 Inversion of three rays with noise versus direction at a frequency of $0.2 \mathrm{~Hz} . \ldots \ldots \ldots \ldots$. . . . . . . . . . . . . . . 149

4.33 Inversion of nine rays with noise versus frequency at direction of 90

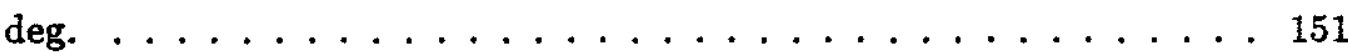

4.34 Inversion of nine rays with noise versus direction at a frequency of

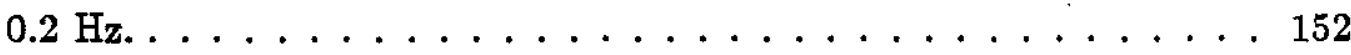

4.35 Variance of the ray inversion solution. . . . . . . . . . . 153

4.36 Variance of the ray inversion solution using a surface wave directional buoy in box 1. . . . . . . . . . . . . . . . . 154

4.37 Normalized resolution of the frequency-direction spectrum in box 2

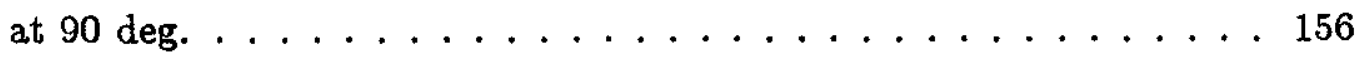

4.38 Normalized resolution of the frequency-direction spectrum in box 5 at $90 \mathrm{deg} \ldots \ldots \ldots \ldots \ldots \ldots \ldots \ldots \ldots \ldots$ 


\section{List of Tables}

2.1 Shallow water waveguide wander and bias. . . . . . . . . . 45

2.2 Shallow water waveguide ray angles and bounces . . . . . . . 45

2.3 Shallow water waveguide wander and bias. . . . . . . . . . 46

2.4 Shallow water ray angles and bounces. . . . . . . . . . . . . 46

2.5 Arctic waveguide wander and bias, $b=.1$ and $c_{0}=1440 \mathrm{~m} / \mathrm{s}$. . . . 51

2.6 Arctic waveguide equivalent surface ray angles and number of bounces, $b=.1$ and $c_{0}=1440 \mathrm{~m} / \mathrm{s} . \ldots \ldots 51$

2.7 Arctic waveguide wander and bias, $b=.016$ and $c_{0}=1449.2 \mathrm{~m} / \mathrm{s} . \ldots 52$

2.8 Arctic waveguide equivalent surface ray angles and number of bounces, $b=.016$ and $c_{0}=1449.2 \mathrm{~m} / \mathrm{s} \ldots \ldots \ldots 52$

2.9 Mode eigenvalues for $180 \mathrm{~m}$ ideal waveguide at $50 \mathrm{~Hz}$. . . . . . 61

2.10 Coupled pressure magnitude, phase and arrival time for modes 1-3. . 64

2.11 Coupled pressure magnitude, phase and arrival time for modes 4-6. . 65

2.12 Coupled pressure magnitude, phase and arrival time for modes 7-9. . 66

2.13 Coupled pressure magnitude, phase and arrival time for modes 10-12. 68

2.14 Coupled pressure magnitude, phase and arrival time for modes 1-3. . 70

2.15 Coupled pressure magnitude, phase and arrival time for modes 4-6. . 71

2.16 Coupled pressure magnitude, phase and arrival time for modes 7-9. . 72

2.17 Coupled pressure magnitude, phase and arrival time for modes 10-12. 74 
2.18 Coupling strengths into mode 1 for $180 \mathrm{~m}$ waveguide, frequency of $50 \mathrm{~Hz}$, and range of $50 \mathrm{~km} . \ldots \ldots . \ldots 75$ 


\section{List of Symbols}

$A$ acoustic ray amplitude

$A_{m} \quad$ complex amplitude of mode m

$A_{m n}$ first coupling coefficient from mode n to $\mathrm{m}$

$A \quad n^{2}$-linear waveguide gradient

$a_{n}$ acoustic mode normalizaton factor

$B_{m n}$ second coupling coefficient from mode $\mathrm{n}$ to $\mathrm{m}$

$b$ sound speed gradient.

$c$ sound speed

$c_{s}$ sound speed at source

$c_{0}$ sound speed at surface

$c_{p} \quad$ surface wave phase velocity at spectral peak

$c_{j n}$ jth coefficient in power series of $\xi$ for $\epsilon_{n}$

$\mathrm{C}_{2}$ correlation matrix for boxes

$\mathbf{D}_{1}$ finite difference matrix for direction

$d_{j n}$ jth coefficient in power series of $\xi$ for $\Delta \tau_{n}$

$F$ sea surface wave frequency-direction spectrum

$F_{m} \quad$ reduced acoustic radial solution for mode $\mathrm{m}$

f vector of frequency-direction spectra 
G kernal matrix

$\mathrm{G}_{g}^{-1}$ generalized inverse operator

$\tilde{G}_{n}^{o}$ nth mode adiabatic Green's function

$\tilde{G}_{n} \quad$ nth mode Green's function

$g$ acceleration due to gravity at sea level

$H$ waveguide depth

H characteristic Airy waveguide depth

$H_{0}$ background waveguide depth

$h$ sea surface wave spectrum directional model

$\tilde{H}^{o}$ adiabatic Hamiltonian

$I_{n m} \quad$ single scattering coupling integral

$K$ sea surface wavenumber

$k$ acoustic wavenumber

$k_{0}$ acoustic wavenumber at surface

$L$ quadratic functional for model error and smoothness

$M$ number of ray bounces between source and receiver

$n$ acoustic index of refraction

$p$ acoustic pressure

$p_{n}^{\text {adiab }}$ mode $\mathrm{n}$ adiabatic acoustic pressure

$p_{n m}^{\text {coupl }}$ mode $\mathrm{n}$ coupled acoustic pressure from mode $\mathrm{m}$

$R$ range from source to receiver

$\mathrm{R}$ resolution matrix

$r$ range

$\mathrm{S}_{1}$ smoothing matrix for direction 
$\mathrm{S}_{2} \quad$ smoothing matrix for boxes

SL acoustic source power level in $\mathrm{dB}$

$\bar{s}_{n}^{g r} \quad$ background acoustic group slowness of mode $\mathrm{n}$

$\mathrm{TL}_{1}$ acoustic transmission loss in $\mathrm{db}$ due to spreading

$\mathrm{TL}_{2}$ acoustic transmission loss in $\mathrm{db}$ due to scattering

$T_{i j}$ cross coherence matrix entry for acoustic mode $i$ and $j$

$t$ time

$\mathrm{t}$ vector of travel time spectra

$U$ separated acoustic range solution

$U_{n}$ acoustic range dependence of mode $n$

$U_{c}$ wind velocity in direction of surface waves at $10 \mathrm{~m}$ height

$V_{n m}$ coupling potential from mode $\mathrm{n}$ to $\mathrm{m}$

$v_{p}$ phase velocity of surface wave

$v_{p n}^{\text {mode }}$ acoustic phase velocity of mode $\mathrm{n}$

$v_{g n}^{\text {mode }}$ acoustic group velocity of mode $\mathrm{n}$

$v_{g n}^{r a y}$ acoustic group velocity of ray $\mathrm{n}$

W estimation error weighting matrix

$W$ acoustic ray eikonal

$w$ transformed Airy depth

$w_{0}$ transformed Airy depth at surface

$X$ sea surface wave spectrum

$y_{n} \cdot$ nth zero of Airy function $\mathrm{Ai}$

$Z$ separated acoustic depth solution

$Z_{n}$ acoustic eigenfunction of mode $\mathrm{n}$

$\tilde{z}$ complex energy (wavenumber squared) 
$z$ depth

$z_{0}$ depth of acoustic source

$Z$ space-time covariance of sea surface height

$\alpha$ angle measured from perpendicular to acoustic path

$\alpha_{n}$ grating angle locations

$\beta$ sea surface wave directional width parameter

$\eta$ sea surface wave spectrum equilibrium or rear face parameter

$\Delta f$ acoustic signal bandwidth

$\Delta r$ ray skip distance

$\Delta r_{i j k}$ distance from source $\mathrm{i}$ to receiver $\mathrm{j}$ in box. $\mathrm{k}$

$\Delta s_{n}^{g r} \quad$ acoustic group slowness fluctuation of mode $n$

$\Delta \tau_{n}$ acoustic travel time fluctuation of mode $\mathrm{n}$

$\Delta \varphi_{n}$ acoustic spatial phase fluctuation of mode $n$

$\delta_{n, m}$ Kronecker delta function

$\delta$ Dirac delta function

$\epsilon_{n}$ acoustic radial eigenvalue fluctuation

$\hat{\varepsilon}$ error due to frequency-direction component dependence

$\Phi$ sea surface wave frequency spectrum

$\hat{\Phi}$ sea surface wave cross spectrum

$\varphi_{n}$ acoustic spatial phase of mode $n$

$\varphi_{n}^{\text {ray }}$ acoustic spatial phase of ray $\mathrm{n}$

$\bar{\varphi}_{n}$. mean acoustic spatial phase of mode $n$

$\varphi_{n}^{(0)}$ WKB spatial phase of mode $\mathrm{n}$

$\varphi_{n}^{(2)}$ coupled acoustic spatial phase of mode $n$

$\Gamma$ sea surface spectral peak enhancement exponent 
$\gamma \quad$ acoustic vertical wavenumber

$\gamma_{n}$ acoustic vertical eigenvalue of mode $\mathrm{n}$

$\bar{\gamma}_{n}$ background vertical eigenvalue of mode $n$

$\kappa$ acoustic radial wavenumber

$\kappa_{n} \quad$ acoustic radial eigenvalue of mode $\mathrm{n}$

$\bar{\kappa}_{n}$ background acoustic radial eigenvalue of mode $\mathrm{n}$

$\Lambda_{\mathrm{nm}}$ cycle distance for modes $\mathrm{m}$ and $\mathrm{n}$

$\lambda$ acoustic wavelength

$\lambda^{s}$ sea surface wavelength

$\lambda_{1}$ Lagrange multiplier for direction smoothing

$\lambda_{2}$ Lagrange multiplier for box smoothing

II acoustic source power

$\Theta_{n}^{r a y} n$ nth ray phase fluctuation covariance

$\Theta_{n}^{\text {mode }}$ nth mode phase fluctuation covariance

$\theta_{n} \quad$ nth ray angle of incidence

$\vartheta_{n}^{\text {mode }}$ covariance of acoustic mode $n$ phase fluctuations

$\vartheta_{n}^{r a y}$ covariance of acoustic ray n phase fluctuations

$\rho$ density

$\rho_{s}$ density at source

$\varrho \quad$ sea surface frequency spectrum peak enhancement factor

$\sigma \quad$ surface wave spectral peak width parameter

$\sigma_{\phi}$ rms phase error

$\sigma_{\tau} \quad$ rms travel time error

$\sigma_{\Delta \varphi} \quad$ rms acoustic phase wander

$\sigma_{\Delta \tau}$ rms acoustic travel time wander 


$\begin{aligned} \sigma_{\xi} & \text { rms sea surface height } \\ \tau_{n} & \text { acoustic travel time of mode } \mathrm{n} \\ \tau_{n}^{\text {ray }} & \text { acoustic travel time of mode } \mathrm{n} \\ \Upsilon_{n}^{\text {ray }} & \text { spectrum of travel time fluctuation of ray } \mathrm{n} \\ \Upsilon_{n}^{\text {mode }} & \text { spectrum of travel time fluctuation of mode } \mathrm{n} \\ v_{n}^{\text {ray }} & \text { covariance of travel time fluctuation of ray } \mathrm{n} \\ v_{n}^{\text {mode }} & \text { covariance of travel time fluctuation of mode } \mathrm{n} \\ \Omega & \text { sea surface radian frequency } \\ \Omega_{p} & \text { sea surface radian frequency of spectral peak } \\ \omega & \text { acoustic radian frequency } \\ \xi & \text { sea surface height } \\ \psi & \text { particle displacement potential } \\ \zeta & \text { correlation length of sea surface height }\end{aligned}$




\section{Chapter 1}

\section{Introduction}

\subsection{Background}

Ocean acoustic tomography is fast becoming an established tool for remotely sensing the ocean interior $[1,2,3]$. The basis of tomography is the measurement of acoustic signal travel time perturbations due to the sound speed variations that are caused by oceanographic phenomena along the various acoustic paths[4]. Linear inverse techniques are applied to the measurements to estimate these sound speed variations. Acoustic tomography has been successfully employed to study a variety of ocean features such as mesoscale eddies[5,6], currents[7], internal waves[8], and barotropic motions[9].

Until recently, using acoustic tomography to remotely sense the ocean surface has been an unexplored possibility. This thesis introduces a technique that estimates the quasi-stationary and quasi-homogeneous sea surface wave frequency-direction spectrum from the spectra of acoustic travel time or phase fluctuations measured at a number of receivers or transceivers. For acoustic transmissions which interact with the sea surface, information on the surface height is included in the signal 
along with the information on the other internal ocean features. ${ }^{1}$ The time scale of the wind-generated sea surface gravity waves (on the order of $10 \mathrm{sec}$ ) is much shorter than that of most other oceanographic processes (the fastest of which are internal waves whose time scale is on the order of many minutes). Therefore, a spectrum of the fluctuations naturally separates the surface wave effects from the other oceanographic processes.

The motion of the sea surface is a function of space and time. This surface presents the appearance of a series of irregular moving crests and troughs that gradually grow and decay with time. If we neglect spray, air bubbles in the water, and breaking waves, this surface is then a single-valued function of $x, y$, and $t$, which we will denote as $\xi(x, y, t)$. The water at and below $\xi(x, y, t)$ is set into motion by wind. The water motion extracts energy from wind and stores it in the form of kinetic and potential energy. This energy is in turn dissipated by the turbulence below the surface and by wave breaking above. However, the dissipative effects are weaker than the generative effects of the wind. A considerable portion of the wave energy can continue for a number of hours and travel hundreds of kilometers away from the area with strong wind to areas of relative calm.[10]

To a good approximation, the sea surface is Gaussian, locally homogeneous, and short term stationary $[11,12,13,14,15]$. The Gaussian assumption implies that the amplitudes of various spectral (sinusoidal) components are independent. Given a large number of independent wave components, the Gaussian property follows from the Central Limit Theorem[16]. The Gaussian property allows us to describe the sea surface with only the second moment, with all higher moments being derivable from the second. This second moment is called the time-space covariance function and the various spectra are Fourier transforms over the different space and time

\footnotetext{
${ }^{1}$ Of course, this technique is not applicable to acoustic signals which have no interaction with the sea surface, e.g. acoustic rays or modes trapped in the deep sound channel.
} 
variables.[17] The locally homogeneous assumption implies the changing sea state has decorrelation space scales much larger than a surface wavelength. The short term stationary assumption implies that the sea state has decorrelation time scales much longer than a wave period.

Sea surface waves are usually characterized by various spectral descriptors, among which are the frequency spectrum, the spectrum of the time series of sea surface height measured at one spot, the wavenumber spectrum, the two dimensional spatial spectrum of sea height measured at one time, the wave number-frequency spectrum, the three dimensional spectrum of sea height at all time and space, and various spectra in between. Of interest to this thesis is one particular spectrum: the frequency-direction spectrum, a two dimensional spectrum which sorts the wave energy by temporal frequency and by the direction the waves are travelling[18].

Prior to the early 1950's, there were few reliable and consistent methods for observing the sea surface wave field. Since then, many techniques have been developed to measure the various spectra. The measurement techniques for the onedimensional frequncy spectrum can be broken down into two categories: those which directly measure sea surface elevation and those which are based on other properties of the wave field as described in a review of the study of wind waves by Barnett and Kenyon in 1975[17].

In the direct methods, a common technique that is used is to insert a rigid wave staff into the water and measure an electrical property of the staff that is influenced by the amount of the staff immersed in the water. All three basic electrical properties (resistance, capacitance, and inductance) have been utilized but the most successfull has been capacitance wave staffs[19]. The wave staff method is restricted primarily to the laboratory and relatively shallow water because it requires a fixed platform from which to operate.

A second class of devices measure some other property of the wave field be- 
sides height and deduce the height spectrum. Of these, the two most common are pressure tranducers and accelerometers. Pressure tranducers consist of a wire attached to a rigid diaphragm. Changes in pressure due to wave motion act on the diaphragm, changing the tension on the wire[20]. The pressure fluctuations are then transformed into sea height estimates through linear wave theory. Again, this method is restricted to shallow water. Accelerometers, configured in a buoy, measure the vertical acceleration in the wave field. The sea height is related to the acceleration by linear theory. These buoys, sometimes referred to as wave rider buoys, can be deployed in any depth water and have been widely used.

The measurement of two dimensional spectra has advanced in the past few years. In 1960, Cote, et al. obtained the first two dimensional spectrum of the sea surface using aerial photogrammetry[21]. Since, then a number of techniques have been developed which can be broken down into four categories: pitch-roll buoys, wave staff arrays, acoustic backscatter methods, and aircraft-and satellite-based radar.

The pitch-roll buoy and the related cloverleaf buoy measure vertical acceleration, pitch, roll, and heading $[22,12,23,24]$. From these, directional spectra estimates can be obtained, however, the resolution is limited by the fact that only the first few Fourier coefficients of the angular distribution of spectral energy can be determined. Much success has been achieved in measuring two dimensional spectra with wave staff arrays. The work was pioneered by Barber in the early 1950's[25] and was extended by Munk in the early 1960's[26]. An array of wave staffs measure the sea surface elevation at a number of locations to give estimates of the time-space covariance function. The Fourier transform of this covariance function yields an estimate of the two dimensional spectrum. This technique has been used successfully by Donelan and his co-workers to measure directional spectra in Lake Ontario[27]. However, as previously mentioned, this technique is restricted to shallow waters like Lake Ontario because of the need for a fixed platform. Acoustic backscatter 
techniques rely on the transmission of an acoustic pulse underwater and the reflection of that pulse from the underside of the sea surface wave field. The measured Doppler shifts can be used to infer the velocity field near the surface and hence the directional spectrum. $[28,29,30]$ Hill and Farmer [28] have developed a buoy which samples the backscattered acoustic Doppler shifts in azimuth and estimates the local frequency-direction spectrum. These methods have been used successfully but are restricted to measuring the spectrum in one location.

Labianca [31] has developed a technique for estimating the frequency-direction spectrum using a "full wave" inversion method. Labianca proposes measuring the forward-scattered field from a continuous wave (CW) source at a number of receivers in a circular array around the source. The spectrum of scattered waves have sidebands introduced by the interaction with the moving sea surface. The acoustic spectra are then inverted using linear inverse techniques. The Labianca technique is, in some respects, similiar to the method we introduce in this thesis. Both are based on acoustic forward scattering by the sea surface and both use a similiar inversion technique. However, there are important differences. 1) Labianca's technique is restricted to relatively short ranges and a single interaction with the surface, while our technique, as we will show, is useful at much longer ranges and assumes many acoustic interactions with the sea surface. 2) His technique assumes, correctly for his short ranges, that the sea surface is homogeneous while we are able to invert for the inhomogeneous spectra. 3) The Labianca technique depends on frequency effects of the sea surface waves on the acoustic transmissions while we use the phase and travel time effects.

There are a number of methods by which directional wave spectra may be obtained using microwave radar techniques. First, there are those techniques which directly image the surface elevation and take a two-dimensional tranform later. These direct imaging methods are Real Aperture Radar (RAR), Surface Contour 
Radar (SCR), and Synthetic Aperture Radar (SAR)[32]. RAR's map the power backscattered from the ocean surface to fixed side-looking antennas with fine resolution in angle. Range resolution is obtained using very short pulses. The relationship between power received and wave height must be known to map the surface. This relationship is not well known at present. Also, RAR is not usable from satellites because of the need for fine angular resolution. SCR uses a narrow, downward-looking microwave beam scanned perpendicularly to the direction of aircraft travel. Horizontal resolution is obtained by a small illuminated footprint while range resolution is obtained by short pulses. $\mathrm{SCR}$ has been very successfully used to measure sea spectra from aircraft[33]. It is also not usable on satellites because of the need for a small footprint. SAR maps the ocean surface to fine resolution with a fixed, side-looking antenna that simulates a large antenna by continuously scanning while it travels above the ocean surface[34]. Range resolution is obtained with short pulses while azimuthal resolution relies on mapping Doppler shifts into positions on the sea surface. For a moving ocean surface, azimuthal resolution is a complicated function of the surface wave velocities and scattering intensity. However, SAR's from aircraft and satellites have produced spectra that agree with other methods $[35,36,34]$.

A microwave measurement that images in range while it integrates in azimuth is the Remote Ocean-Wave Spectrometer (ROWS). The technique performs a one dimensional transform in the direction the airplane is traveling. ROWS has been developed using both frequency modulation (FM) and amplitude modulation (AM). The FM technique involves illuminating areas of the ocean surface that are small in range extent but large in azimuth and measuring the mean Doppler shift from the surface using the FM part of the coherent return. The large azimuthal extent averages out waves not traveling in the look direction, thus producing directional discrimination. Linear wave theory is used to map the measured velocities into wave 
spectral densities. This technique has been used from aircraft but theoretically can be used from satellites[32]. All of the radar based methods have an inherent $180 \mathrm{deg}$ ambiguity, i.e. they cannot differentiate between.waves traveling in opposite directions. We show in this thesis that our technique suffers the same 180 deg ambiguity. Of course, all the radar based methods need a platform such as an aircraft or satellite to operate from and this can limit the time spent over any one area of the ocean.

With all of the above described techniques, one might ask why bother developing a new method to study surface waves. First, the capabilities of acoustic tomography are now enhanced. Tomography experiments which transmit acoustic signals at fast enough rates (greater than $.25 \mathrm{~Hz}$ ) can now study surface waves besides the other oceanographic phenomena. Second, tomographic experiments can last many weeks and cover hundreds of kilometers of ocean, depending on battery limitations, number of transceivers, etc. This enables the continuous study of waves in a large area of the ocean for that time. Third, tomography can resolve spatial changes in spectra in the covered area. For instance, tomography can estimate surface wave spectra in areas which are fetch-dependent. Fourth, we can integrate other wave spectra measuring systems (such as buoys or satellites) into the tomographic inversion producing a better overall estimate. Fifth, and most important we feel, tomography enables the simultaneous measurement of surface wave spectra and other oceanographic features such as currents and internal waves. Surface wave/current interactions and surface wave/internal wave interactions[37,38] are being studied and we hope that tomography might be able to assist in those investigations. 


\subsection{Thesis Overview}

This thesis is divided into five chapters. Chapter 2 describes the effect of the rough sea surface on acoustic modes and rays. Modes and rays are the standard descriptors of acoustic propagation in the ocean. Fluctuations in the local height of the ocean cause fluctuations in the arrival times and phase of modes and rays. The relationship between sea height and travel time fluctuation is developed. Besides this shifting (or "wandering") in time, the shape of the acoustic pulse can be distorted (or "spread"). Coupled mode theory is used to assess the accuracy of the wander-only or adiabatic approximation, upon which our inversion scheme depends.

Chapter 3 first gives an overview of surface wave spectral theory. There, the spectrum of the acoustic travel time and phase fluctuations is shown to be related to the frequency-direction spectrum of the sea surface when the wave spectrum is homogeneous. Chapters 2 and 3 together solve the forward problem. In fact, in the homogeneous case described in Chapter 3, the frequency-directional spectrum is shown to be an algebraic transformation of the measured acoustic phase and travel time spectra. We show that, like radar-based methods, this technique also suffers from a $180 \mathrm{deg}$ ambiguity problem. The theory developed in this chapter is applied to data measured on two days in the 1984 Marginal Ice Zone Experiment (MIZEX '84) in the Greenland Sea off Spitzbergen. Frequency spectra were estimated that are related to wind force data measured on the two days.

Chapter 4 addresses the tomographic inverse problem for the frequency-direction spectrum when the spectrum is spatially inhomogeneous. We solve the inverse problem using a variant of the damped least-squares technique. This technique involves the physically justified assumption of smoothness constraints on the frequencydirection spectrum spatially and in direction. It is shown that only one inverse operation need be carried out in these two dimensions, and the resulting general- 
ized inverse operator applied at each frequency of interest. The effectiveness of this technique is investigated using synthetic surface wave spectra based on a model by Donelan[27]. The synthetic acoustic spectra are measured at a number of tomographic tranceivers and then the generalized inverse operator is applied to the measured acoustic spectra to estimate the spatially dependent frequency-direction spectrum. Results are described for both modes and rays. The effect of noisy measurements is quantified. The results using noisy data are shown to agree with theoretical variance estimates from inverse theory. Resolution issues associated with the inverse operator are also discussed. Finally, Chapter 5 summarizes the findings of this thesis.

This thesis is multi-disciplinary and, as such, deals with different quantities which are traditionally represented in the literature by the same variables. An example is radial frequency of acoustic waves and radial frequency of surface waves, usually represented by $\omega$. To prevent confusion, we have redefined a number of variables in this thesis, e.g. $\omega$ remains acoustic frequency while we have chosen $\Omega$ to represent surface wave frequency. A List of Symbols is provided on page 14 that might be useful to the reader. 


\section{Chapter 2}

\section{Acoustic Scattering by a Rough Sea Surface}

This thesis is concerned with tomographic inversion of the travel times or phases of acoustic signals for sea surface wave spectra. Hence, we will mainly concentrate on rough surface effects on signal travel times and phases. To a lesser degree, the effects on amplitude will be also touched upon, but only in the context of predicting signal-to-noise ratios and pulse spreading effects which might degrade the time or phase measurement.

In this chapter, we will describe the effect of the rough sea surface on acoustic normal modes (Section 2.1) and rays (Section 2.2), which are standard descriptors of the acoustic field in the ocean.

Normal modes constitute a "full wave" solution to the wave equation in the ocean waveguide. Fluctuations in the local height of the ocean waveguide cause fluctuations in the arrival times and phases of the normal modes which have a turning point at the surface. If the slopes of the surface waves are small, the coupling between modes can be taken to be negligible. Conditions for the validity of this approximation (called the adiabatic approximation [39]) are presented. The adiabatic approximation implies that, at the receiver, the mode arrival only shifts (or "wanders") in time but that the shape of the arriving pulse is not distorted (or "spread") by energy coupled from other modes. Our inversions for sea surface 
spectra depend on the adiabatic approximation where arrival time fluctuations are uncontaminated by pulse distortion effects.

Rays are the paths of acoustic energy obtained when the frequency of the transmitted sound is assumed infinite[40]. We also relate the fluctuations in ray arrival times and phases to the sea surface roughness. We assume the ray pulse wanders, but is undistorted by scattering with the surface in regions where the adiabatic mode approximation holds valid.

Finally, in Section 2.3, we summarize the results of this chapter that we need to use in Chapters 3 and 4.

\subsection{Normal Modes}

Here, we concentrate on rough surface effects on the propagation of acoustic normal modes. We start with a overview of mode theory in a horizontally stratified ocean in Section 2.1.1, followed by the generalization to a range dependent ocean in Section 2.1.2. We concentrate on the phase and travel time variations due to the rough surface in Section 2.1.3 where we assume the adiabatic and WKB approximations. The variations are dependent on the background waveguide characteristics such as depth, sound speed profile, etc. We use two canonical examples, an ideal waveguide and an $n^{2}$-linear profile, for illustration of the rough surface effects in Section 2.1.4. The analysis is used to explain the high mode coherence measured in the FRAM IV experiment in Section 2.1.5

Coupled mode effects are addressed in Section 2.1.6 where we give a solution for coupled mode pressure based on a single scattering approximation. Bragg scattering is discussed in Section 2.1.7 that involves the matching of the surface wavelength and and mode cycle distance. The validity of the adiabatic approximation is discussed. Also, in Section 2.1.9, the validity of the WKB approximation is quantified. 

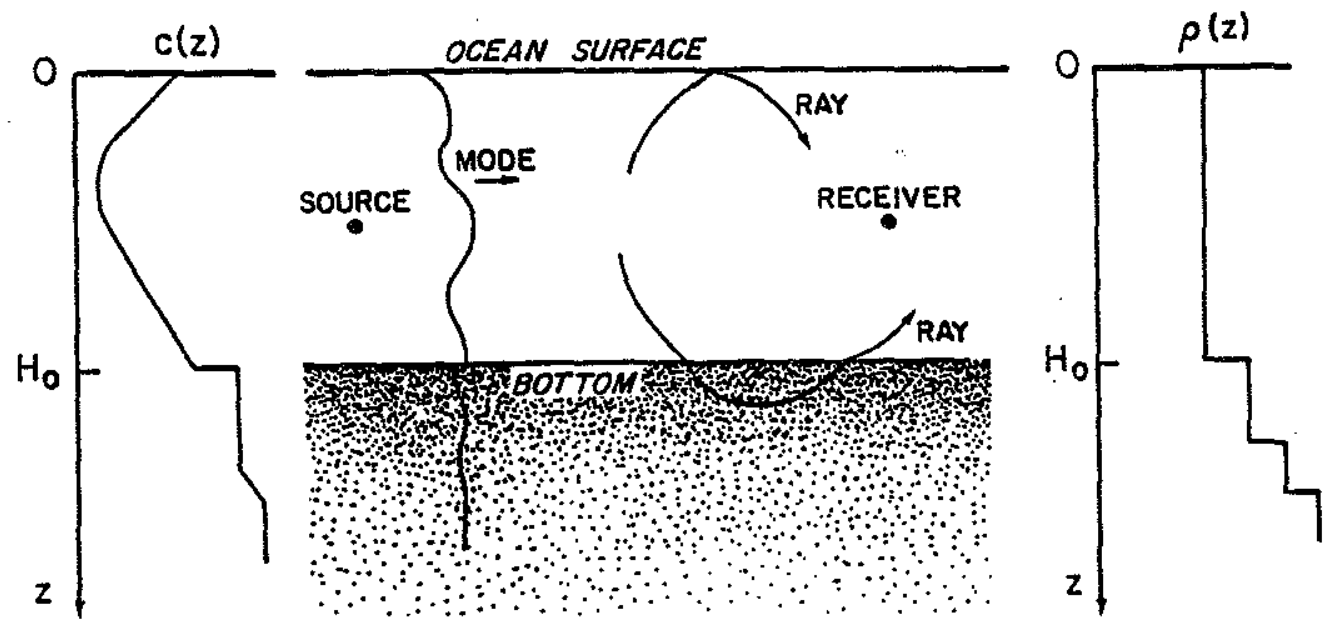

Figure 2.1: Horizontally stratified waveguide.

\subsubsection{Normal Modes in a Stratified Ocean .}

The general horizontally stratified problem in underwater acoustics is the one illustrated in Fig. 2.1. The ocean waveguide is of a constant depth $H$ with a sound speed $c(z)$ and density $\rho(z)$, constant in range but not in depth. The top boundary is a pressure release surface, i.e. $p(r, z=0)=0$. The bottom is also a horizontally stratified medium. The source is located at $z=z_{0}$ and $r=0$ with an acoustic power of $\Pi$. Our goal is to find the pressure field due to this source at all $z$ and $r$.

We describe the acoustic pressure as a sum of normal modes. It is convenient to introduce a particle displacement potential $\psi$, in terms of which the pressure is given by

$$
p=-\rho \frac{\partial^{2} \psi}{\partial t^{2}}
$$

The three-dimensional wave equation (see $[41]$ for derivation) for the displacement 
potential is

$$
\nabla^{2} \psi=\frac{1}{c^{2}} \frac{\partial^{2} \psi}{\partial t^{2}}
$$

For a harmonic source $e^{-i \omega t}, p=\omega^{2} \rho \psi$, and the wave equation becomes the Helmholtz equation

$$
\nabla^{2} \psi+\frac{\omega^{2}}{c^{2}} \psi=0
$$

For sources on the $z$ axis, the field is symmetrical about the $z$ axis and the wave equation in cylindrical coordinates becomes

$$
\frac{\partial^{2} \psi}{\partial r^{2}}+\frac{1}{r} \frac{\partial \psi}{\partial r}+\frac{\partial^{2} \psi}{\partial z^{2}}+\frac{\omega^{2}}{c^{2}} \psi=0
$$

To solve Eq. (2.4), we assume a separable solution. $\psi \sim U(r) Z(z)$. Equation (2.4) then becomes

$$
\frac{U^{\prime \prime}(r)+\frac{1}{r} U^{\prime}(r)}{U(r)}+\frac{Z^{\prime \prime}(z)}{Z(z)}-\frac{\omega^{2}}{c^{2}}=0
$$

where the prime denotes differentiation with respect to the variable in the parentheses. The separated equations become

$$
\begin{gathered}
U^{\prime \prime}(r)+\frac{U^{\prime}(r)}{r}+\kappa^{2} U(r)=0 \\
Z^{\prime \prime}(z)+\gamma^{2} Z(z)=0
\end{gathered}
$$

where we define the wavenumber as

$$
k^{2} \equiv \frac{\omega^{2}}{c^{2}}
$$

and the separation constants are related by

$$
\kappa^{2}+\gamma^{2}=k^{2}
$$

Equation (2.6) is Bessel's equation of the zeroth order. Its solution is the cylindrical Bessel function of zeroth order $J_{0}(\kappa r)$. For the far field, where $\kappa r \gg 1$, we can expand the Bessel function taking only the outgoing wave, so that

$$
U(r) \simeq \frac{1}{\sqrt{2 \pi \kappa r}} e^{i(\kappa r-\pi / 4)}
$$


The amplitude of $U(r)$ and therefore $\psi$ decrease as $r^{1 / 2}$ and the phase dependence is the same for all depths at a constant range.

If the harmonic source is located at a depth $z_{0}$, we can write the pressure in the waveguide as a product of the $Z_{m}, U_{m}$, and $e^{-i \omega t}[41]$

$$
p(r, z, t)=e^{-i\left(\omega t+\frac{\pi}{4}\right)} \sum_{m} \frac{q_{m}}{\sqrt{\kappa_{m} r}} Z_{m}\left(z_{0}\right) Z_{m}(z) e^{i \kappa_{m} r}
$$

where

$$
\begin{aligned}
& q_{m}=\frac{2 \pi \rho}{\nu_{m}} \sqrt{\rho_{s} c_{s} \Pi} \\
& \nu_{m}=\int_{0}^{\infty} \rho_{s} Z_{m}^{2}(z) d z
\end{aligned}
$$

where $c_{s}$ and $\rho_{s}$ is the sound speed and density at the source depth, and $\rho$ is the density at the receiver. Equation (2.11) is valid for any horizontally stratified waveguide without attenuation. However, the evaluation of the eigenvalues is, in general, not analytic except for a few cases such as the hard bottom, constant sound speed wavguide or the $n^{2}$-linear waveguide; both will be discussed further on.

\subsubsection{Normal Modes in a Range Dependent Ocean}

When the ocean is not horizontally stratified, the Helmholtz equation (Eq. (2.3)) is no longer separable, and we have to turn to coupled mode theory to solve for the pressure field. Coupled normal mode theory for a range dependent ocean was developed independently by Pierce[42] in 1965 and Milder[43] in 1969. An excellent account of coupled mode theory can be found in a $\mathrm{PhD}$ thesis by Rutherford[39].

Rewriting Eq. (2.3) for a range dependent waveguide,

$$
\nabla^{2} \psi(r, z)+k^{2}(r, z) \psi(r, z)=0
$$

with the appropriate boundary conditions. The range dependence can occur in two different ways. The sound speed, and therefore the wavenumber, can be a function 
of range. But even with otherwise stratified sound speed profiles, the boundary conditions on $\psi$ may be a function of range as it is with a rough sea surface. The boundary and wavenumber conditions on $\psi$ might also involve the range-dependence of density[39], but we will not discuss density variations further.

Pierce[42] postulated a solution to Eq. (2.14) that was partially separated. i.e.

$$
\psi(r, z)=\sum_{n} U_{n}(r) Z_{n}(z ; r)
$$

where now the depth functions $Z_{n}$ are range-dependent as well as depth-dependent. Equation (2.7) then becomes

$$
\frac{\partial^{2} Z_{n}(z ; r)}{\partial z^{2}}+\left[k^{2}(r, z)-\kappa_{n}^{2}(r)\right] Z_{n}(z ; r)=0
$$

The boundary conditions which the $Z_{n}$ must satisfy are that $\rho Z_{n}$ and the normal component of particle velocity, $\nabla_{\perp} Z_{n}$, be continuous across a boundary. For a rough boundary defined by $z=\xi(r), \nabla_{\perp}$ is given by

$$
\begin{aligned}
\nabla_{\perp} & =\frac{1}{\sqrt{1+\dot{\xi}}}\left(\frac{\partial}{\partial z}-\dot{\xi} \frac{\partial}{\partial r}\right) \\
\dot{\xi} & =\frac{d \xi(r)}{d r} .
\end{aligned}
$$

The first step in solving this coupled mode problem is to substitute the trial solution Eq. (2.15) into the Helmholtz equation (Eq. (2.14)) which gives

$$
\sum_{n}\left[\dot{U}_{n} Z_{n}+2 \dot{U}_{n} \dot{Z}_{n}+\frac{U_{n} \dot{Z}_{n}}{r}+\frac{\dot{U}_{n} Z_{n}}{r}+U_{n} \bar{Z}_{n}+U_{n}\left(\frac{\partial^{2} Z_{n}}{\partial z^{2}}+k^{2}(r, z) Z_{n}\right)\right]=0
$$

where the dot denotes differentiation with respect to $r$. The depth functions $Z_{n}(z ; r)$ form an orthonormal set at each range $r$ such that

$$
\int_{0}^{\infty} \rho Z_{n}(z ; r) Z_{m}(z ; r) d z=\delta_{n, m}
$$


If Eq. (2.18) is multiplied by $\rho Z_{m}(z ; r)$ and integrated over depth, we get

$$
\ddot{U}_{m}(r)+\frac{1}{r} \dot{U}_{m}(r)+\kappa_{m}^{2}(r) U_{m}(r)=-\sum_{n \neq m}\left[A_{m n} U_{n}+B_{m n}\left(\frac{U_{n}}{r}+2 \dot{U}_{n}\right)\right]
$$

where the coupling coefficients $A_{m n}$ and $B_{m n}$ are given by

$$
\begin{aligned}
& A_{m n}(r)=\int_{0}^{\infty} \rho(z) Z_{m}(z ; r) \ddot{Z}_{n}(z ; r) d z \\
& B_{m n}(r)=\int_{0}^{\infty} \rho(z) Z_{m}(z ; r) \dot{Z}_{n}(z ; r) d z .
\end{aligned}
$$

Often, a more convenient "reduced" differential equation is obtained by the substitution of

$$
U_{m}(r)=\frac{F_{m}(r)}{\sqrt{r}}
$$

into Eq. (2.20). The reduced radial wave equation then becomes

$$
\ddot{F}_{m}+\left(\kappa_{m}(r)+\frac{1}{4 r^{2}}\right) F_{m}(r)=-\sum_{n \neq m}\left(A_{m n} F_{n}+2 B_{m n} \dot{F}_{n}\right) .
$$

The $\frac{1}{4 r^{2}}$ term can be neglected except within a few wavelengths of the source.

The adiabatic approximation consists of setting $A_{m n}$ and $B_{m n}$ to zero in Eq. (2.24), i.e.

$$
\bar{F}_{m}(r)+\kappa_{m}^{2}(r) F_{m}(r)=0 .
$$

The difference between the range-dependent Eq. (2.25) and the range-independent Eq. (2.6) is that the horizontal wavenumber $\kappa_{m}$ is now a function of position. The spatial phase term from Eq. (2.11), $\kappa_{m} r$, must be modified to account for the range dependence. A standard approximation to make here is to assume that the spatial phase has the form $\int_{0}^{R} \kappa_{m}\left(r^{\prime}\right) d r^{\prime}$. This approximation is known as the WKB (Wentzel-Kramers-Brillouin) approximation, and is valid when the variation in the properties of the medium per wavelength is small[40].

In the depth equation boundary conditions, dropping $\dot{\xi}$ terms in Eq. (2.18) gives

$$
\nabla_{\perp} \simeq \frac{\partial}{\partial z}
$$


making the depth equation (2.16) as easy to solve as the range independent case except we have to solve it at all ranges of interest to get the horizontal wavenumber $\kappa_{n}(r)$; moreover, we need the eigenfunction $Z_{n}(z ; r)$ at source and receiver. The range-varying horizontal wavenumber also appears inside the square root portion of Eq. (2.11). This amplitude change is much less important than the phase change and an average or background value for the wavenumber (denoted by $\bar{\kappa}_{n}$ ) can be used there. From here on in, the time dependence, the $\pi / 4$ phase shift, and the normalization term $q_{m}$ will be taken as implicit, so that the acoustic pressure becomes

$$
p(r, z)=\sum_{n} \frac{1}{\sqrt{\kappa_{n} r}} Z_{n}\left(z_{0} ; 0\right) Z_{n}(z ; r) e^{i \int_{0}^{r} \kappa_{n}\left(r^{\prime}\right) d r^{\prime}} .
$$

In this form, one sees explicitly that the modal eigenvalues are a function of range, and it is through the $\kappa_{n}\left(r^{\prime}\right)$, as well as through the mode functions $Z_{n}$ at the source and receiver, that one introduces the effects of roughness. For a particular realization of a rough surface, which we will consider throughout this thesis to be "frozen" during the time of transmission of a single pulse, the adiabatic approximation to the pressure field can be expressed as

$$
p(r, z)=\sum_{n} \frac{1}{\sqrt{\kappa_{n} r}} Z_{n}\left(z_{o} ; 0\right) Z_{n}(z, r) e^{i R_{n} r+i \int_{0}^{r} \epsilon_{n}\left(r^{\prime}\right) d r^{\prime}}
$$

where we have broken $\kappa_{n}\left(r^{\prime}\right)$ into the sum of an average, range independent background component $\bar{\kappa}_{n}$, and a range dependent wavenumber fluctation component, $\epsilon_{n}\left(r^{\prime}\right)$. The amplitude, phase, and group velocity fluctations of the pressure due to boundary roughness can be determined in this adiabatic context by specifying a surface height distribution function and then taking the appropriate moments of Eq. (2.28). 


\subsubsection{Phase and Travel Time Variations}

The data used in acoustic tomography are the travel time variations of an acoustic arrival. In this thesis, we are concerned with using acoustic tomography to deduce information on the ocean surface height distribution In this section, we show the connection between the surface height depth variations and modal phase and travel time variations.

Phase The phase of an acoustic normal mode, under the WKB approximation, from Eq. (2.28), is

$$
\varphi_{n}=\bar{\kappa}_{n} R+\int_{0}^{R} \epsilon_{n}(r) d r
$$

We define

$$
\Delta \varphi_{n} \equiv \int_{0}^{R} \epsilon_{n}(r) d r
$$

as the phase variation. The range dependence of the eigenvalue is assumed to be constant in time for the time-of-flight of the mode, i.e. we assume the "frozen" approximation. The implications of the frozen approximation are discussed later in Section 3.6. Fig. 2.2 illustrates an adiabatic mode adapting to the change in sea surface height.

The covariance of the phase variation at times $t_{1}$ and $t_{2}$ corresponding to two different transmissions is given by

$$
\vartheta_{n}^{\text {mode }}\left(t_{1}, t_{2}\right)=<\Delta \varphi_{n}\left(t_{1}\right) \Delta \varphi_{n}\left(t_{2}\right)>
$$

where $\langle f\rangle$ denotes the expected value of $f$.

We can write the eigenvalue variation as a power series in the sea surface height

$$
\epsilon_{n}\left(r_{1}, t_{1}\right)=\sum_{j} c_{j n} \xi^{j}\left(r_{1}, t_{1}\right)
$$

where the coefficients $c_{j n}$ are dependent on the background waveguide sound speed profile. The $c_{j n}$ are explicitly calculated for $j=1,2$ in Section 2.1.4. Convergence 


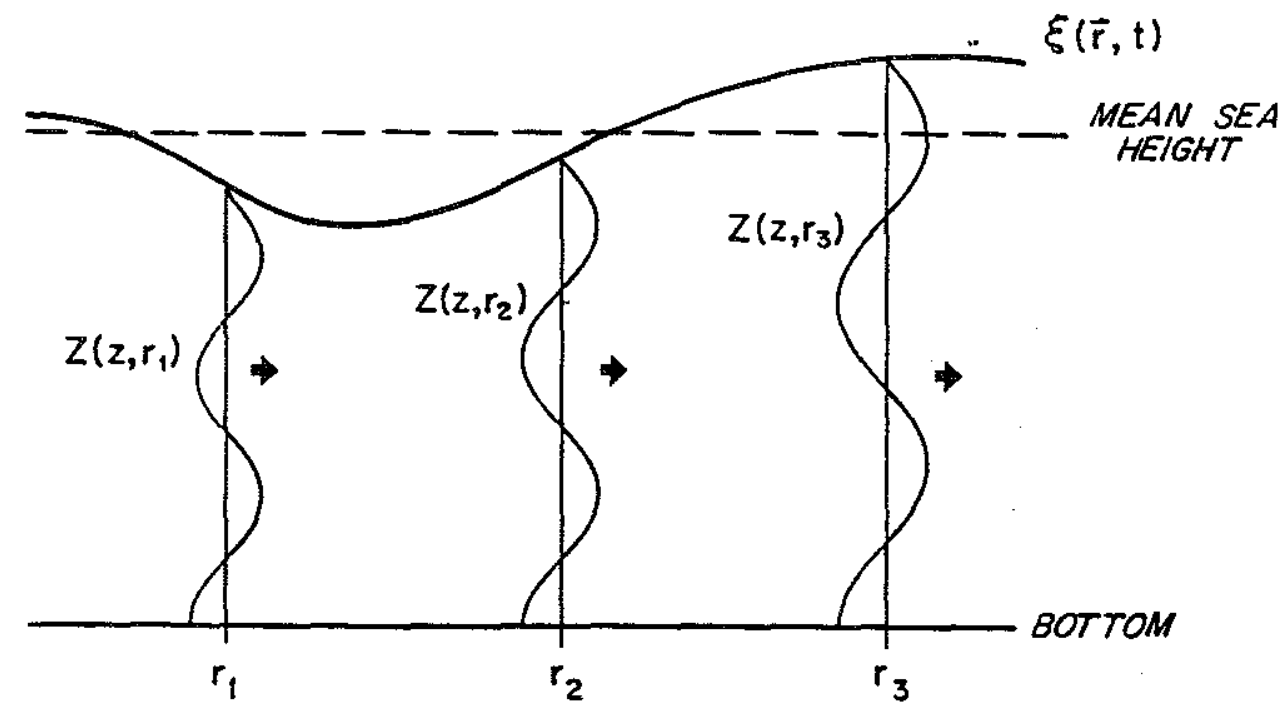

Figure 2.2: Adiabatic mode adapting to changes in sea surface height.

issues for this series will not be discussed except to note that $c_{1 n} \xi \gg c_{\text {in }} \xi^{j}$ for $j \geq 2$ is necessary to be able to write

$$
\Delta \varphi_{n}\left(t_{1}\right) \simeq c_{1 n} \int \xi\left(r_{1}, t_{1}\right) d r_{1}
$$

Note that we also have assumed that the background waveguide is range independent. If the background waveguide is range dependent and the adiabatic approximation still holds, the problem is more complicated but solvable. For example, the $c_{1 n}$ become $c_{1 n}\left(r_{1}\right)$ and must remain within the integral in Eq. (2.33). If the background waveguide is very range dependent, mode coupling can make the problem very difficult. Using Eq. (2.33) in Eq. (2.31), the phase variation covariance to first order becomes

$$
\vartheta_{n}^{\text {mode }}\left(t_{1}, t_{2}\right)=c_{1 n}^{2}<\int_{0}^{R} \xi\left(r_{1}, t_{1}\right) d r_{1} \int_{0}^{R} \xi\left(r_{2}, t_{2}\right) d r_{2}>
$$

If the sea surface statistics are stationary in time and homogeneous in space, Eq. 
(2.34) can be written as

$$
\vartheta_{n}^{\text {mode }}(t)=c_{1 n}^{2} \int_{0}^{R} \int_{0}^{R} Z(r, t) d r_{1} d r_{2}
$$

where now $t=t_{1}-t_{2}, r=r_{1}-r_{2}$, and $Z(r, t)$ is the space-time covariance of the sea surface. The variance of the surface displacement is $\sigma_{\xi}^{2}=Z(0,0)$. Since $Z(r, t)$ is dependent only on the difference of $r_{1}$ and $r_{2}$, the double integral in Eq. (2.35) can be rewritten as [16]

$$
\vartheta_{n}^{\text {mode }}(t)=c_{1 n}^{2} \int_{-R}^{+R}(R-|r|) Z(r, t) d r .
$$

We can approximate Eq. (2.36) at $t=0$ to get an estimate of the rms "wander" of the phase variation, i.e.

$$
\sigma_{\Delta \varphi_{n}} \simeq c_{1 n} \sigma_{\xi} \sqrt{2 R_{\zeta}}
$$

where $\zeta$ is the correlation length of the zero mean sea surface height. If we write the phase variation to second order, we get

$$
\Delta \varphi_{n}=\int_{0}^{R}\left(c_{1 n} \xi+c_{2 n} \xi^{2}\right) d r
$$

Taking the expected value of Equation(2.38) and assuming a zero mean surface,

$$
\left\langle\Delta \varphi_{n}\right\rangle \simeq R c_{2 n} \sigma_{\xi}^{2}
$$

Therefore, to second order in surface height, phase variation is not zero-mean but has a bias given by Eq. (2.39). Figure 2.3 illustrates the effects of wander and bias schematically. These are similiar to the effects described by Flatte[8] due to internal waves.

Travel Time We now examine the mode travel time fluctuations due to the rough surface. The travel time $\tau_{n}$ of a normal mode at frequency $\omega_{0}$ is given by stationary phase arguments as

$$
\left.\frac{\partial}{\partial \omega}\left[\varphi_{n}(r, \omega)-\omega \tau_{n}\right)\right]_{\omega_{0}}=0
$$




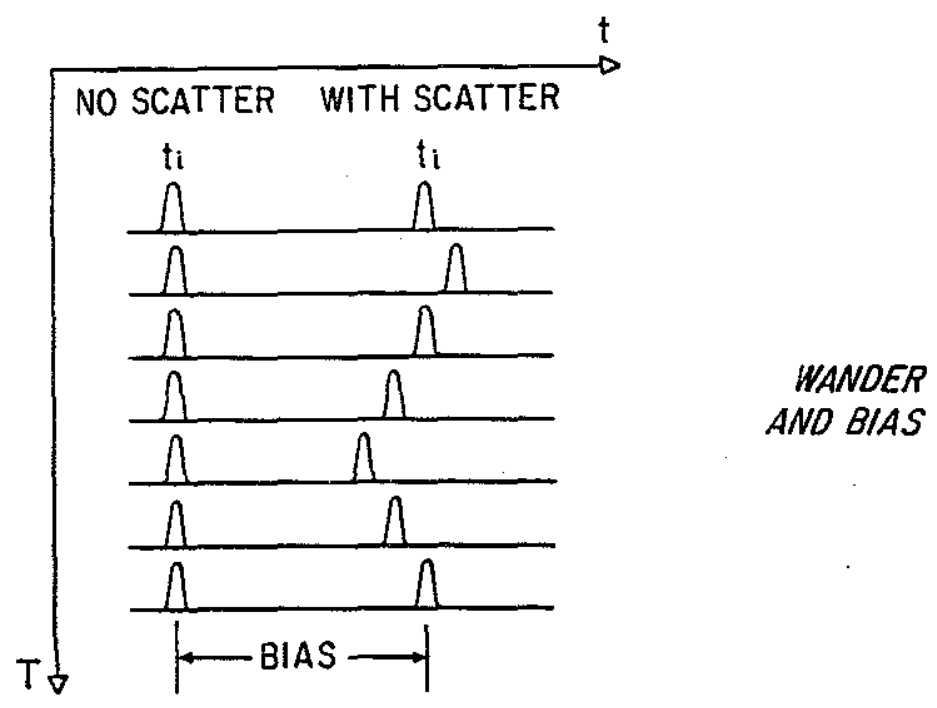

Figure 2.3: Travel time wander and bias due to surface waves.

or

$$
\tau_{n}=\left.\frac{\partial \varphi_{n}}{\partial \omega}\right|_{\omega_{0}}
$$

If we now include a fluctuation component in the phase, as discussed before, we obtain

$$
\Delta \tau_{n}=\frac{\partial}{\partial \omega} \Delta \varphi_{n}=\int_{0}^{R} \frac{\partial \epsilon_{n}}{\partial \omega} d r
$$

where $\frac{\partial \epsilon_{n}}{\partial \omega} \equiv \Delta s_{n}^{g r}$ is the group slowness (inverse of the group velocity) variation. Similiar to the modal phase, the travel time variation can be written as a variation in the surface height $\xi$, i.e.

$$
\Delta \tau_{n}\left(t_{1}\right)=\int_{0}^{R} d_{1 n} \xi\left(r_{1}, t_{1}\right) d r+O\left(\frac{\xi^{2}}{H^{2}}\right)
$$

where $H$ is the characteristic waveguide depth and $d_{1 n}=\partial c_{1 n} / \partial \omega$ (which is derived for two canonical waveguides in Section 2.1.4). We can write the relationship between the travel time covariance and the space-time covariance function of the sea surface as

$$
v_{n}^{\text {mode }}(t)=<\Delta \tau_{n}\left(t_{1}\right) \Delta \tau_{n}\left(t_{2}\right)>
$$


or, following the arguments used for the phase,

$$
v_{n}^{\text {mode }}(t)=d_{1 n}^{2} \int_{-R}^{+R}(R-|r|) Z(r, t) d r
$$

As above, we can approximate Eq. (2.45) at $t=0$ to get an estimate of the rms "wander" of the travel time variation, i.e.

$$
\sigma_{\Delta \tau_{n}} \simeq d_{1 n} \sigma_{\xi} \sqrt{2 R \delta}
$$

where $\delta$ is the correlation length of the zero mean sea surface. If we write the travel time variation to second order, we get

$$
\Delta \tau_{n}=\int_{0}^{R}\left(d_{1 n} \xi+d_{2 n} \xi^{2}\right) d r
$$

Taking the expected value of Eq. (2.47) and assuming a zero mean surface displacement, we get the "bias" of the travel time variations

$$
\left\langle\Delta \tau_{n}\right\rangle \simeq R d_{2 n} \sigma_{\xi}^{2}
$$

\subsubsection{Canonical Waveguide Fluctuation Parameters}

This section describes the phase and travel time fluctuations due to a rough surface for two canonical waveguides: the ideal hard bottom shallow water waveguide and an $n^{2}$-linear waveguide which emulates the upward refracting conditions found in the Arctic.

Ideal Hard Bottom Shallow Water Waveguide The ideal waveguide we will consider here is the so called "hard-bottom" waveguide [40] shown in Fig. 2.4 in which the compressional wave speed and density in the bottom halfspace go to infinity. The water column is isovelocity, and the waveguide is range independent 


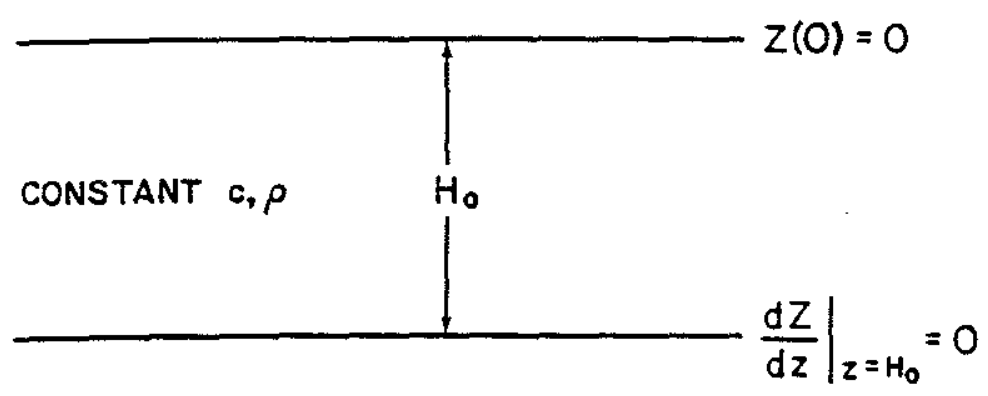

Figure 2.4: Ideal hard bottom shallow water waveguide

except for the air-water interface roughness. For the background waveguide (no surface roughness effects included), the vertical mode functions $Z_{n}(z)$ are given by

$$
Z_{n}(z)=a_{n} \sin \bar{\gamma}_{n} z
$$

where the vertical wavenumber $\bar{\gamma}_{n}$ and the horizontal wavenumber $\bar{\kappa}_{n}$ are given by

$$
\bar{\gamma}_{n}=\frac{\left(n-\frac{1}{2}\right) \pi}{H_{0}}
$$

and

$$
\vec{\kappa}_{n}=\sqrt{\kappa^{2}-\bar{\gamma}_{n}^{2}}=\sqrt{\left(\frac{\omega}{c}\right)^{2}-\bar{\gamma}_{n}^{2}}
$$

where $H_{0}$ is the depth of the water column. The normalization factor $a_{n}$ can be found by substituting Eq. (2.49) into the orthonormality condition given by Eq. (2.19) which gives $a_{n}=\sqrt{\frac{2}{\rho H_{0}}}$. To include the effects of surface roughness, we add a variation $\xi\left(r_{1}\right)$ to the water depth $H_{0}$ in Eq. (2.51) to obtain

$$
\kappa_{n}\left(r_{1}\right)=\sqrt{\left(\frac{\omega}{c}\right)^{2}-\frac{\left(n-\frac{1}{2}\right)^{2} \pi^{2}}{\left(H_{0}+\xi\left(r_{1}\right)\right)^{2}}} .
$$


If we assume that $H_{0} \gg \xi\left(r_{1}\right)$ for all $r_{1}$, then we can expand the $\left(H_{0}+\xi\left(r_{1}\right)\right)^{-2}$ term and the square root to get

$$
\kappa_{n}=\bar{\kappa}_{n}\left[1+\frac{\bar{\gamma}_{n}^{2} \xi}{\bar{\kappa}_{n}^{2} H_{0}}-\left(\frac{3 \bar{\gamma}_{n}^{2}}{2 \bar{\kappa}_{n}^{2}}+\frac{\bar{\gamma}_{n}^{4}}{2 \bar{\kappa}_{n}^{4}}\right)\left(\frac{\xi}{H_{0}}\right)^{2}+\ldots\right] .
$$

The variation in the horizontal wavenumber,

$$
\epsilon_{n}\left(r_{1}\right) \equiv \kappa_{n}\left(r_{1}\right)-\vec{\kappa}_{n}
$$

which from Eq. (2.53) gives

$$
\epsilon_{n}=\frac{\bar{\gamma}_{n}^{2}}{\bar{\kappa}_{n}}\left[\frac{\xi}{H_{0}}-\left(\frac{3}{2}+\frac{\bar{\gamma}_{n}^{2}}{2 \bar{\kappa}_{n}^{2}}\right)\left(\frac{\xi}{H_{0}}\right)^{2}+\ldots\right] .
$$

We saw that to second order, $\epsilon_{n}=c_{1 n} \xi+c_{2 n} \xi^{2}$ so that for the ideal shallow water waveguide

$$
c_{1 n}=\frac{\bar{\gamma}_{n}^{2}}{\bar{\kappa}_{n} H_{0}}
$$

and

$$
c_{2 n}=-\frac{\bar{\gamma}_{n}^{2}}{\bar{\kappa}_{n} H_{0}^{2}}\left(\frac{3}{2}+\frac{\bar{\gamma}_{n}^{2}}{2 \bar{\kappa}_{n}^{2}}\right) .
$$

The relative importance of the wander and bias of the phase variation can be assessed by looking at the ratio of the first two terms in the series expansion for the eigenvalue variation

$$
\left|\frac{c_{2 n} \xi^{2}}{c_{1 n} \xi}\right|=\left(\frac{3}{2}+\frac{\gamma_{n}^{2}}{2 \kappa_{n}^{2}}\right) \frac{\xi}{H_{0}} .
$$

The ratio in Eq. (2.58) tells us that there are two ways for the bias to become important. First, for any mode, a large changes in the surface height can increase the bias. Second, for any mode with a large vertical wavenumber, (i.e. with $\gamma_{n} \gg \kappa_{n}$ ) very small changes in the surface height can have a drastic impact on the bias. It will be shown later that the coupling also becomes very important for modes with large $\gamma_{n}$ or when the changes in the surface height become large, so the adiabatic approximation is not adequate to describe the propagation. But when the adiabatic 
approximation does hold, the bias is much less than the wander for reasonable acoustic ranges.

The group slowness variation is to second order $\Delta s_{n}^{g r}=d_{1 n} \xi+d_{2 n} \xi^{2}$. For the shallow water waveguide

$$
d_{1 n}=\frac{\partial c_{1 n}}{\partial \omega}=-\frac{\bar{s}_{n}^{g r} \bar{\gamma}_{n}^{2}}{H_{0} \bar{\kappa}_{n}^{2}}
$$

and

$$
d_{2 n}=\frac{3 \vec{\gamma}_{n}^{2} \bar{s}_{n}^{g r}}{2 \bar{\kappa}_{n}^{2} H_{0}^{2}}\left(1+\frac{\bar{\gamma}_{n}^{2}}{\bar{\kappa}_{n}^{2}}\right)
$$

where $\bar{s}_{n}^{g r}=\frac{\omega}{\pi_{n} c^{2}}$ is the unperturbed group slowness for this waveguide. We can calculate a ratio of bias to wander terms for group slowness in the same manner as we did for the phase, i.e.

$$
\left|\frac{d_{2 n} \xi^{2}}{d_{1 n} \xi}\right|=\frac{3}{2}\left(1+\frac{\bar{\gamma}_{n}^{2}}{\bar{\kappa}_{n}^{2}}\right) \frac{\xi}{H_{0}}
$$

Similiar comments can be made about the importance of the travel time bias as for the phase.

At this point, it would be useful to consider two examples. The rms wander and bias of the phase and travel time can be calculated for simple example waveguides using Eqs. (2.37), (2.39), (2.46) and (2.48). For a frequency of $220 \mathrm{~Hz}$, surface correlation length of $100 \mathrm{~m}$, rms surface height of $1 \mathrm{~m}$ and sound speed of $1500 \mathrm{~m} / \mathrm{s}$, Tables 2.1 and 2.3 lists the wander and bias estimates of the phase and travel time for 5 modes. Table 2.1 pertains to a $30 \mathrm{~m}$ waveguide and range of $5 \mathrm{~km}$, while Table 2.3 is for a waveguide depth of $180 \mathrm{~m}$ and a range of $50 \mathrm{~km}$. Tables 2.2 and 2.4 list the equivalent ray angles $\theta_{m}$ and number of equivalent ray surface bounces $M$ for the modes[44]. The equivalent ray angles and the number of surface bounces are higher for higher modes. Thus the wander and bias are expected to increase with higher mode number because of the increased $\frac{\gamma_{n}}{\kappa_{n}}$ as the results in Tables 2.1 to 2.4 show. Also, modes in the $180 \mathrm{~m}$ waveguide are affected less than the modes in 


\begin{tabular}{||c|c|c|c|c||}
\hline $\mathrm{n}$ & $\sigma_{\Delta \varphi_{n}}(\mathrm{rad})$ & $\left\langle\Delta \varphi_{n}\right\rangle(\mathrm{rad})$ & $\sigma_{\Delta \tau_{n}}(\mathrm{~ms})$ & $\left\langle\Delta \tau_{n}\right\rangle(\mathrm{ms})$ \\
\hline 1 & .10 & -.02 & .07 & .02 \\
\hline 2 & .91 & -.23 & .68 & .17 \\
\hline 3 & 2.6 & -.67 & 2.0 & .55 \\
\hline 4 & 5.3 & -1.4 & 4.6 & 1.4 \\
\hline 5 & 9.4 & -2.6 & 9.2 & 3.1 \\
\hline
\end{tabular}

Table 2.1: Shallow water waveguide wander and bias, depth of $30 \mathrm{~m}$, range of $5 \mathrm{~km}$.

\begin{tabular}{||c|c|r||}
\hline $\mathrm{n}$ & $\theta_{m}(\mathrm{deg})$ & $M$ \\
\hline 1 & 3 & 5 \\
\hline 2 & 10 & 14 \\
\hline 3 & 17 & 25 \\
\hline 4 & 23 & 36 \\
\hline 5 & 31 & 50 \\
\hline
\end{tabular}

Table 2.2: Shallow water waveguide equivalent ray angle and number of bounces, depth of $30 \mathrm{~m}$, range of $5 \mathrm{~km}$.

the $30 \mathrm{~m}$ waveguide. Another interesting result from the Tables is the fact that the phase and travel time bias are indeed much less than the wander. In addition, the travel time bias is positive, i.e. the arrival times are longer than we would expect from a smooth surface. The magnitude of the phase wander in Tables 2.1 and 2.3 indicate that only modes 1 and 2 for the $30 \mathrm{~m}$ waveguide and modes numbers less than about 17 for the $180 \mathrm{~m}$ waveguide could be coherently averaged[4] for SNR gain (as is commonly done in tomography). Coherent averaging needs rms wander much less than $90 \mathrm{deg}$ and only the modes mentioned meet that condition.

$n^{2}$-Linear (Arctic) Waveguide Here we follow Brekovskikh [40], who derives analytic expressions for the mode eigenvectors and eigenvalues for an " $n^{2}$-linear" waveguide. If we note that, to first approximation, the Arctic can be modeled as 


\begin{tabular}{||c|c|c|c|c||}
\hline $\mathrm{n}$ & $\sigma_{\Delta \varphi_{n}}(\mathrm{rad})$ & $\left\langle\Delta \varphi_{n}\right\rangle(\mathrm{rad})$ & $\sigma_{\Delta r_{n}}(\mathrm{~ms})$ & $\left\langle\Delta \tau_{n}\right\rangle(\mathrm{ms})$ \\
\hline 1 & .001 & -.0002 & .001 & .0001 \\
\hline 5 & .12 & -.02 & .09 & .01 \\
\hline 9 & .43 & -.06 & .32 & .04 \\
\hline 13 & .93 & -.13 & .72 & .10 \\
\hline 17 & 1.7 & -.23 & 1.3 & .20 \\
\hline
\end{tabular}

Table 2.3: Shallow water waveguide wander and bias, depth of $180 \mathrm{~m}$, range of $50 \mathrm{~km}$.

\begin{tabular}{||l|c|c|}
\hline $\mathrm{n}$ & $\theta_{m}(\mathrm{deg})$ & $M$ \\
\hline 1 & .5 & 1 \\
\hline 5 & 5 & 12 \\
\hline 9 & 9 & 23 \\
\hline 13 & 14 & 34 \\
\hline 17 & 18 & 46 \\
\hline
\end{tabular}

Table 2.4: Shallow water waveguide equivalent ray angles and number of bounces, depth of $180 \mathrm{~m}$, range of $50 \mathrm{~km}$. 
isothermal $\left(0^{\circ} \mathrm{C}\right)$ and isohaline (35 $\left.\frac{\circ}{\circ o}\right)$, then the sound speed is linear with depth $z$,

$$
c(z)=c_{0}+b z
$$

where $c_{0}=1449.2 \mathrm{~m} / \mathrm{s}$ and $b=0.016 \mathrm{~m} / \mathrm{s} / \mathrm{m}$ is the sound speed gradient. For the “ $n^{2}$-linear" waveguide, we write

$$
c(z)=\frac{c_{0}}{\sqrt{1-a z}}
$$

which, for $a z \ll 1$, can be approximated by

$$
c(z)=c_{0}\left(1+\frac{1}{2} a z+\ldots\right)
$$

To match the approximate psuedolinear profile in Eq. (2.64) and the linear profile in Eq. (2.62), we set $a=\frac{2 b}{c_{0}}=2.21 \times 10^{-5} m^{-1}$. Due to the smallness of $a$, the expression of Eq. (2.63) can approximate the linear Arctic model for thousands of meters of depth.

Turning to the modal solution of the wave equation, we first write the vertical Helmholtz equation,

$$
\frac{d^{2} Z(z)}{d z^{2}}+\left[\kappa_{0}^{2}(1-a z)-\kappa_{n}^{2}\right] Z(z)=0
$$

where $\kappa_{0}=\frac{\omega}{c_{0}}$ and $\kappa_{n}$ is the horizontal wavenumber. By introducing the change of variables

$$
\begin{aligned}
H & =\frac{1}{\sqrt[3]{a \kappa_{0}^{2}}} \\
w_{0} & =\mathcal{H}^{2}\left(\kappa_{n}^{2}-\kappa_{0}^{2}\right) \\
w & =w_{0}+\frac{z}{\mathcal{H}}
\end{aligned}
$$

where $H$ is the characteristic waveguide thickness, Eq. (2.65) reduces to the Airy equation

$$
\frac{d^{2} Z(w)}{d w^{2}}=w Z(w)
$$


The Airy functions $\mathrm{Ai}(w)$ and $\mathrm{Bi}(w)$ are solutions to $\mathrm{Eq}$. (2.66). Retaining only the $\operatorname{Ai}(w)$ solution for which $\operatorname{Ai}(w) \rightarrow 0$ as $w \rightarrow \infty$, and imposing a pressure release boundary condition $\mathrm{Ai}\left(w_{0}\right)=0$ at the surface, we obtain the normal modes by finding the zeroes of the Airy function Ai at $z=0$, i.e.

$$
w_{0}=-y_{n} \quad n=1,2,3 \ldots
$$

where $y_{1}=2.338, y_{2}=4.088$, etc[45]. The modal eigenvalue is then

$$
\kappa_{n}^{2}=\kappa_{0}^{2}-\frac{y_{n}}{\mathscr{H}^{2}}
$$

To include the effects of boundary roughness, we require that the pressure release boundary condition be met not at $w=w_{0}$, but rather at $w=w_{0}-\frac{\xi}{\not y}$, i.e.

$$
Z_{n}\left(w_{0}-\frac{\xi}{\not H}\right)=0
$$

This requirement leads to the modified eigenvalue equation

$$
\begin{aligned}
\kappa_{n} & =\sqrt{\kappa_{0}^{2}-\frac{y_{n}}{\mathcal{H}^{2}}+\frac{\xi}{\not^{3}}} \\
& =\sqrt{\bar{\kappa}_{n}^{2}+\frac{\xi}{\not^{3}}}
\end{aligned}
$$

where $\bar{\kappa}_{n}$ is, as before, the undisturbed waveguide eigenvalue. For $\frac{\xi}{\not} \ll 1$ (generally a good assumption), we can do our usual expansion of the root and solve for the eigenvalue variation up to second order

$$
\epsilon\left(r_{1}, t_{1}\right)=\frac{a \kappa_{0}^{2} \xi\left(r_{1}, t_{1}\right)}{2 \bar{\kappa}_{n}}-\frac{a^{2} \kappa_{0}^{4} \xi^{2}\left(r_{1}, t_{1}\right)}{8 \bar{\kappa}_{n}^{3}} .
$$

For this case,

$$
c_{1 n}=\frac{a \kappa_{0}^{2}}{2 \vec{\kappa}_{n}}
$$

and

$$
c_{2 n}=-\frac{a^{2} \kappa_{0}^{4}}{8 \bar{\kappa}_{n}^{3}}
$$


are the first power and second power coefficients of $\xi$ used in this thesis. As we did for the ideal shallow water waveguide, we can compute the relative importance of the bias and wander terms with the ratio

$$
\left|\frac{c_{2 n} \xi^{2}}{c_{1 n} \xi}\right|=\frac{\xi}{\kappa_{n}^{2} \nvdash^{3}} .
$$

The larger the surface height, the larger the bias compared to the wander. However, the effect of higher mode number on the ratio is harder to interpret. Since we are using the $n^{2}$-linear waveguide to approximate the Arctic for modes with turning points removed from the bottom, the effect of the surface height change is much less dependent on mode number for these modes. The mode just moves up and down in the waveguide as the surface moves up and down.

The group slowness variation is, to second order

$$
\begin{aligned}
\Delta s_{n}^{g r} & =\frac{\partial \epsilon_{n}}{\partial \omega} \\
& =\frac{a \omega \xi}{c_{0}^{2} \bar{\kappa}_{n}}\left[1-\frac{\omega \bar{s}_{n}^{g r}}{2 \bar{\kappa}_{n}}\right]-\frac{a^{2} \omega^{3} \xi^{2}}{2 c_{0}^{4} \bar{\kappa}_{n}^{3}}\left[1-\frac{3 \omega \bar{s}_{n}^{g r}}{4 \bar{\kappa}_{n}}\right]
\end{aligned}
$$

where

$$
\bar{s}_{n}^{g r}=\frac{1-\frac{2 y_{n}}{3 \kappa_{0}^{2} y^{2}}}{c_{0} \sqrt{1-\frac{y_{n}}{\kappa_{0}^{2} y^{2}}}}
$$

is the unperturbed group slowness. The coefficients of $\xi$ and $\xi^{2}$ are

$$
d_{1 n}=\frac{a \omega}{c_{0}^{2} \bar{\kappa}_{n}}\left[1-\frac{\omega \bar{s}_{n}^{g r}}{2 \bar{\kappa}_{n}}\right]
$$

and

$$
d_{2 n}=-\frac{a^{2} \omega^{3}}{2 c_{0}^{4} \bar{\kappa}_{n}^{3}}\left[1-\frac{3 \omega \bar{s}_{n}^{g r}}{4 \bar{\kappa}_{n}}\right] .
$$

From Eqs. (2.79) and (2.80), the scattered arrivals are seen to be retarded in arrival time compared to modes traveling under a smooth sea surface. For this waveguide, the ratio of the bias and wander terms is

$$
\left|\frac{d_{2 n} \xi^{2}}{d_{1 n} \xi}\right| \simeq \frac{\xi}{\kappa_{n}^{2} \not^{3}}
$$


which is the same as the ratio for the phase. The wander is dominant over bias for realistic frequencies, surface heights, and sound speed profiles.

Again, it is useful to consider two examples here. The rms wander and bias of the phase and travel time can be calculated for the example waveguides using Eqs. (2.37), (2.39), (2.46) and (2.48). For a frequency of $220 \mathrm{~Hz}$, surface correlation length of $100 \mathrm{~m}$, rms surface height of $1 \mathrm{~m}$, and range of $50 \mathrm{~km}$, Tables 2.5 and 2.7 list the wander and bias estimates of the phase and travel time for 5 modes. Table 2.5 pertains to a waveguide with sound speed gradient $b=.1$ and $c_{0}=1440 \mathrm{~m} / \mathrm{s}$, while Table 2.7 is for a waveguide with purely adiabatic sound speed gradient $b=.016$ and $c_{0}=1449.2 \mathrm{~m} / \mathrm{s}$. These values correspond to the gradients in the waveguide measured during the FRAM IV Experiment as shown in Fig. 2.5. Modes trapped in the upper part of the duct see a gradient $b=.1$ while modes that turn in the lower region mostly see the adiabatic gradient $b=.016$. Tables 2.6 and 2.8 list the equivalent ray angle at the surface $\theta_{m 0}$ and number of equivalent ray surface bounces $M$. Tables 2.5 to 2.8 for the $n^{2}$-linear waveguide give strikingly different results than Tables 2.1 to 2.4 for the shallow water waveguide. The phase and travel time bias and wander are pretty much independent of mode number for the $n^{2}$-linear waveguide. The equivalent ray angles increase with mode number for this waveguide, but the number of bounces decrease by about the same factor as the angles increase. Hence, the independence of the fluctuations with mode number. Another way of stating the above is that the phase of the modes are very highly correlated, as will be shown in Section 2.1.5. The other feature that is evident from the tables is the much lower magnitude of the wander and bias compared with the ideal shallow water waveguide. As we mentioned above, the bottom turning points of all modes are in the water. The effect of moving the surface up or down just moves the mode up or down in the waveguide, giving a very small change in the local eigenvalue as evidenced in the tables. A rough sea surface affects modes the 


\begin{tabular}{||c|c|c|c|c||}
\hline $\mathrm{n}$ & $\sigma_{\Delta \varphi_{n}}(\mathrm{rad})$ & $\left\langle\Delta \varphi_{n}\right\rangle(\mathrm{mrad})$ & $\sigma_{\Delta \tau_{n}}(\mathrm{~ms})$ & $\left\langle\Delta \tau_{n}\right\rangle(\mu \mathrm{s})$ \\
\hline 1 & .21 & -1.2 & .15 & -.08 \\
\hline 2 & .21 & -1.2 & .15 & -.08 \\
\hline 3 & .21 & -1.2 & .15 & -.08 \\
\hline 4 & .21 & -1.2 & .15 & -.08 \\
\hline 5 & .21 & -1.2 & .15 & -.08 \\
\hline
\end{tabular}

Table 2.5: Arctic waveguide wander and bias, $b=.1$ and $c_{0}=1440 \mathrm{~m} / \mathrm{s}$.

\begin{tabular}{||c|c|c|}
\hline $\mathrm{n}$ & $\theta_{\mathrm{m} 0}(\mathrm{deg})$ & $M$ \\
\hline 1 & 4.6 & 22 \\
\hline 2 & 6.1 & 16 \\
\hline 3 & 7.1 & 14 \\
\hline 4 & 7.9 & 13 \\
\hline 5 & 8.1 & 12 \\
\hline
\end{tabular}

Table 2.6: Arctic waveguide equivalent surface ray angles and number of bounces, $b=.1$ and $c_{0}=1440 \mathrm{~m} / \mathrm{s}$.

most when the modes are trapped by the large sound speed discontinuity at the bottom. Modes that turn in the water are less affected by the sea surface.

\subsubsection{Cross-Coherence of Modes in the Arctic}

In his Ph.D. thesis, Polcari[46] computed the cross-coherence matrix of the acoustic normal modes measured in the FRAM IV experiment in the central Arctic. In this section, we would like to show a straightforward application of our theoretical Arctic waveguide results from Section 2.1.4 to explain the high crosscoherence results Polcari found for the modes in this strongly surface scattering Arctic waveguide. 


\begin{tabular}{||c|c|c|c|c||}
\hline $\mathrm{n}$ & $\sigma_{\Delta \varphi_{n}}(\mathrm{rad})$ & $\left\langle\Delta \varphi_{n}\right\rangle(\mu \mathrm{rad})$ & $\sigma_{\Delta \tau_{n}}(\mathrm{~ms})$ & $\left\langle\Delta \tau_{n}\right\rangle(\mu \mathrm{s})$ \\
\hline 1 & .033 & -3 & .02 & -.02 \\
\hline 11 & .034 & -3 & .02 & -.02 \\
\hline 21 & .034 & -3 & .02 & -.02 \\
\hline 31 & .034 & -3 & .02 & -.02 \\
\hline 41 & .034 & -3 & .02 & -.02 \\
\hline
\end{tabular}

Table 2.7: Arctic waveguide wander and bias, $b=.016$ and $c_{0}=1449.2 \mathrm{~m} / \mathrm{s}$.

\begin{tabular}{||l|c|r||}
\hline $\mathrm{n}$ & $\theta_{m 0}(\mathrm{deg})$ & $M$ \\
\hline 1 & 2.5 & 6 \\
\hline 11 & 6.1 & 3 \\
\hline 21 & 7.5 & 2 \\
\hline 31 & 8.6 & 2 \\
\hline 41 & 9.5 & 2 \\
\hline
\end{tabular}

Table 2.8: Arctic waveguide equivalent surface ray angles and number of bounces, $b=.016$ and $c_{0}=1449.2 \mathrm{~m} / \mathrm{s}$. 
The elements of the cross-coherence matrix are defined as

$$
\tau_{i j}=\frac{\left\langle A_{i} A_{j}^{*}\right\rangle}{\sqrt{\left\langle\left|A_{i}\right|^{2}\right\rangle\left\langle\left|A_{j}\right|^{2}\right\rangle}}
$$

where the $i^{\text {th }}$ normal mode is represented as $A_{i}=\left|A_{i}\right| e^{i \varphi_{i}}$. Assuming that only the phase $\varphi_{i}$ of the mode is random, Eq. (2.82) becomes

$$
\tau_{i j}=\left\langle e^{i\left(\varphi_{i}-\varphi_{j}\right)}\right\rangle
$$

The assumption that only the phase of a mode is random can be justified by looking at Eq. (2.28). Note that a variation in a waveguide will affect three parts of that equation. First, and least important, is the $\sqrt{\kappa_{m} r}$ term in the denominator. The eigenvalue fluctuations that are caused by a rough sea surface are generally less than $1 \%$, giving a variation in this term, and hence, the amplitude, of less than $.1 \%$. The second effect of the rough surface is in the eigenfunctions $Z_{m}$ evaluated at the source and receiver. For a mode away from cutoff and mode nulls away from source/receiver depths the variation in eigenfunction amplitude is also small for reasonable surface wave heights and ocean depths. The third effect is in the spatial phase term. Since the variation in phase depends on the integral of the eigenvalue variation, even small variations in the surface height can have dramatic effects on the phase.

We now assume as before that $\varphi_{i}=\bar{\varphi}_{i}-\Delta \varphi_{i}$ where $\Delta \varphi_{i}$ is the small random variation due to the rough surface. For $\Delta \varphi_{i} \ll 1$, we obtain that

$$
\left|\tau_{i j}\right| \simeq 1-\frac{1}{2}\left\langle\Delta \varphi_{i}^{2}\right\rangle-\frac{1}{2}\left\langle\Delta \varphi_{j}^{2}\right\rangle+\left\langle\Delta \varphi_{i} \Delta \varphi_{j}\right\rangle
$$

where we have assumed that $\left\langle\Delta \varphi_{i}\right\rangle=0$.

In Section (2.1.4), we described the scattering in a waveguide with a $n^{2}$-linear sound speed profile characteristic of the Arctic. For this type of waveguide, Eq. (2.84) 


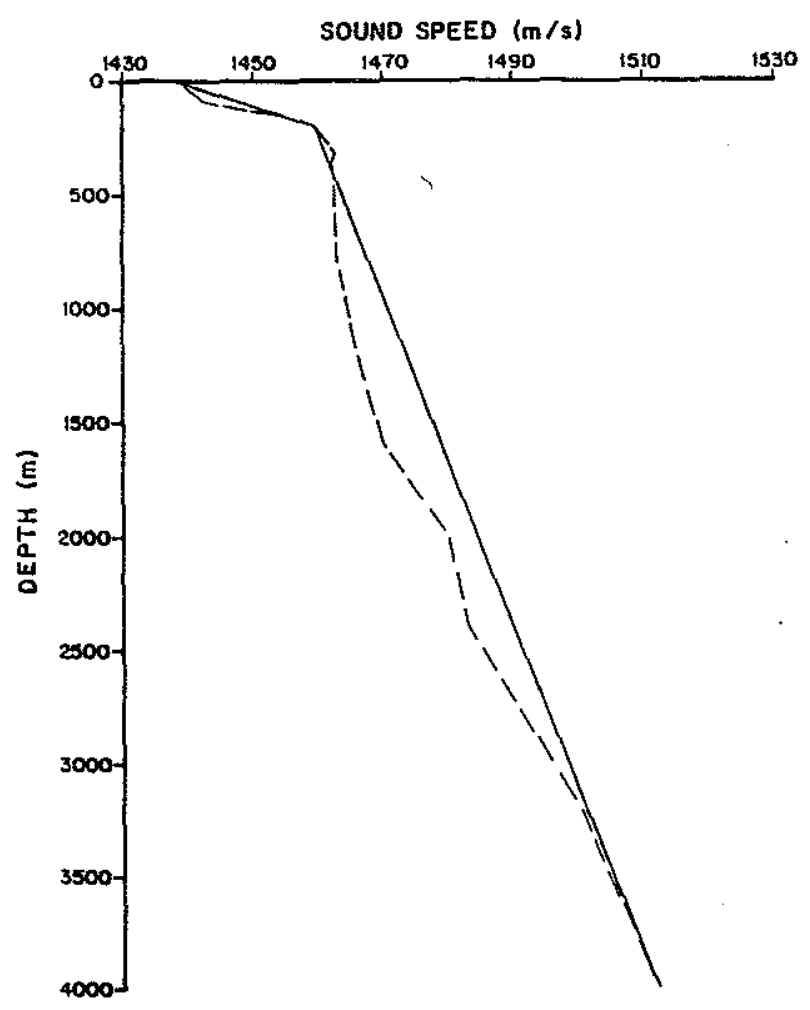

Figure 2.5: FRAM IV sound speed profile and bi-linear approximation

becomes

$$
\left|\tau_{i j}\right| \simeq 1+\frac{a^{2} \omega^{2} \sigma_{\xi}^{2} R_{\zeta}}{2 c_{0}^{4}}\left[\bar{v}_{p i}^{\text {mode }} \bar{v}_{p j}^{\text {mode }}-\frac{1}{2}\left(\bar{v}_{i}^{\text {ph } 2}+\bar{v}_{j}^{\text {ph } 2}\right)\right]
$$

where $\zeta$ is the correlation length of the surface roughness $[47]$ and

$$
\bar{v}_{p i}^{\text {mode }}=\frac{c_{0}}{\sqrt{1-\frac{y_{i}}{\left(\kappa_{0} \not\right)^{2}}}}
$$

is the unperturbed phase velocity of the $i^{\text {th }}$ mode. Eq. (2.85) thus becomes

$$
\left|\tau_{i j}\right| \simeq 1-\frac{a^{2} \sigma_{\xi}^{2} R_{\zeta}\left(y_{i}+y_{j}\right)}{8 \mathcal{H}^{2}} .
$$

The FRAM IV sound speed profile is shown in Fig. (2.5) along with a bilinear approximation. Modes trapped entirely in the upper duct see a linear sound speed profile with gradient of $0.1 \mathrm{~s}^{-1}$ giving $a=1.4 \times 10^{-4}$ in Eq. (2.87). Polcari computed $T_{i j}$ at frequencies from $15-30 \mathrm{~Hz}$ for a transmission range of $300 \mathrm{~km}$ finding typical values for the cross-coherence of low modes of .99 . Assuming a frequency of $30 \mathrm{~Hz}$, 
$\sigma_{\xi}=2 \mathrm{~m}$ and $\zeta=40 \mathrm{~m}$ for the ice roughness and correlation distance, we obtain from our formalism

$$
\left|\tau_{12}\right| \simeq .99987
$$

in very good agreement with Polcari's data analysis. This result implies that the modes turning at the surface wander together as the rough surface changes in time. We note that the theory employed above assumes a pressure-release rough surface, whereas the data was taken under a ice-covered surface - however, this is not expected to significantly change the cross-coherence results calculated here.

There is another implication of the above result: even if modes are not resolvable, (as is often the case at tomographic frequencies in the Arctic) we should be able to see a mode group wander due to the rough surface. The phase perturbations on adjacent modes are very nearly the same, so that the whole arrival pattern is rotated in phase and shifted in time. Using unresolved mode groups might enhance the viability of the proposed technique for estimating sea surface wave spectra with modes.

\subsubsection{Coupled Mode Effects}

If the acoustic waveguide boundaries are range dependent to a great enough degree, the coupling coefficients $A_{m n}$ and $B_{m n}$ given in Eqs. (2.21) and (2.22) may not be small. Since $A_{m n}$ is proportional to the second derivative of the roughness and $B_{m n}$, the first derivative, $B_{m n}$ is assumed to be much greater than $A_{m n}$. We thus drop $A_{m n}$ from here on in this thesis. Most of this section is taken from a paper by Lynch, Miller, Chiu, and Frisk[47].

We now assume that the modal pressure field is made up of two parts: an 
adiabatic part and a coupled part, i.e.

$$
p_{n}=p_{n}^{\text {adiab }}+\sum_{m \neq n} p_{n m}^{\text {coupl }}
$$

where $p_{n}^{a d i a b}$ is given by Eq. (2.25) and our goal in this section is to determine $p_{n m}^{\text {coupl }}$ due to a rough sea surface.

To include coupling effects, we begin by considering two Green's functions, $\tilde{G}$ and $\tilde{G}^{\circ}$. The Green's function $\tilde{G}$ is taken to be the exact solution to Eq. (2.3), i.e. including mode coupling effects. The Green's function $\tilde{G}^{\circ}$, on the other hand, is taken to be the adiabatic mode solution in our paper. (Of course, one can make any choice for $\tilde{G}^{o}$. The choice of the adiabatic $\tilde{G}^{o}$ is attractive for physical reasons.) Mathematically, we can write this in quantum mechanical notation as[48]

$$
\begin{aligned}
\tilde{G}^{o}(\tilde{z}) & =\left(\tilde{z}-\tilde{H}^{o}\right)^{-1} \\
\tilde{G}(\tilde{z}) & =(\tilde{z}-\tilde{H})^{-1}
\end{aligned}
$$

where $\tilde{z}$ is a complex energy (wavenumber squared), $\tilde{H}^{\circ}$ is the Hamiltonian for the adiabatic "background" problem, and

$$
\tilde{H}=\tilde{H}^{\circ}+V
$$

is the Hamiltonian in our "exact" problem. It is obvious from Eq. (2.92) that $V$ will be the mode coupling potential (due to the rough surface) for our modal problem.

The equation relating $\tilde{G}$ and $\tilde{G}^{o}$ is well known, and is called the resolvent equation or Lippmann-Schwinger equation for $\tilde{G}(\tilde{z})$. It is

$$
\tilde{G}(\tilde{z})=\tilde{G}^{o}(\tilde{z})+\tilde{G}^{o}(\tilde{z}) V \tilde{G}(\tilde{z}) .
$$

A common tactic is to solve Eq. (2.93) iteratively, i.e. replace $\tilde{G}(\tilde{z})$ on the right hand side of Eq. (2.93) by the full expression on the right hand side. Doing so, one obtains the infinite series result

$$
\tilde{G}(\tilde{z})=\tilde{G}^{o}(\tilde{z})+\tilde{G}^{o}(\tilde{z}) V \tilde{G}^{o}(\tilde{z})+\tilde{G}^{o}(\tilde{z}) V \tilde{G}^{o}(\tilde{z}) V \tilde{G}^{o}(\tilde{z})+\cdots
$$


This result is called the Born or multiple scattering series. It is an exact series expansion solution to Eq. (2.3) in powers of the coupling potential $V$, but can only be expected to converge rapidly when $V$ is in some sense "weak".

To obtain the coupling potential $V$, we again look at the coupled radial equations, which in reduced form are

$$
\ddot{F}_{n}(r)+\kappa_{n}^{2}(r) F_{n}(r)=-\sum_{m \neq n} 2 B_{n m} \dot{F}_{m}(r)
$$

It is obvious from the form of Eq. (2.95) that, if we had $F_{m}$ instead of $\dot{F}_{m}$ on the right hand side, then $B_{n m}$ would be the coupling potential $V$ for the LippmannSchwinger equation. In order to get this form, one can replace $F_{m}$ by its adiabatic form and take the radial derivative. One obtains, using the WKB solution, that

$$
B_{n m} \dot{F}_{m}=B_{n m} \frac{\partial}{\partial r}\left[\frac{e^{i \int \kappa_{m}(r) d r}}{\sqrt{\kappa_{m}(r)}}\right] .
$$

Using the (generally very good) approximation that $2 \kappa_{n}^{2}(r) \gg \frac{d \kappa_{n}(r)}{d r}$, we write that

$$
B_{n m} \dot{F}_{m} \simeq B_{n m} i \kappa_{m}(r) F_{m}(r)
$$

which leads us to make the identification for the Lippman-Schwinger potential

$$
V_{n m} \longrightarrow B_{n m} i \kappa_{m}
$$

The coupling coefficient $B_{n m}$ for rough surface scattering has been obtained in a convenient form by McDaniel[49,50], and for our $z=0$ surface, we can just paraphrase her results. For the air-water interface at $z=0$, one obtains

$$
B_{m n}=\frac{d \xi}{d r} \frac{2}{\kappa_{n}^{2}-\kappa_{m}^{2}}\left[\frac{\partial Z_{m}}{\partial z} \frac{\partial Z_{n}}{\partial z}\right]_{z=0} .
$$

In Eq. (2.99), $\frac{d \xi}{d r}$ is the local slope of the $z=0$ boundary at a given range $r$, and $Z_{m}$ and $Z_{n}$ are the values of the mode functions at $z=0$ (which are non-zero due to boundary scattering). 
We assume that the sound speed over a small depth near $z=0$ is isovelocity so that we may write the vertical mode functions near $z=0$ as[51]

$$
Z_{n}(z)=a_{n} \sin \gamma_{n} z
$$

and therefore

$$
\left.\frac{\partial Z_{n}}{\partial z}\right|_{z=0}=a_{n} \gamma_{n}
$$

Using the previous results, we can write an explicit form for the single forward scattering term of the series for the $z=0$ scattering surface. It is

$$
\begin{aligned}
p_{n}^{\text {coupl }}(z, R)= & \frac{Z_{n}(z, R)}{\sqrt{\kappa_{m}} \bar{R}} \sum_{m \neq n}\left[\left.\int_{0}^{R} e^{i \int_{r_{1}}^{R} \kappa_{n}\left(r^{\prime \prime}\right) d r^{\prime \prime}} a_{n} \gamma_{n} \frac{d \xi}{d r}\right|_{r_{1}}\right. \\
& \left.\frac{2 i}{\left(\kappa_{n}^{2}-\kappa_{m}^{2}\right)} a_{m} \gamma_{m} e^{i \int_{0}^{r_{1}} \kappa_{m}\left(r^{\prime}\right) d r^{\prime}} d r_{1}\right] Z_{m}\left(z_{o}, 0\right) .
\end{aligned}
$$

The integrals in Eq. (2.102) are fairly easy to interpret physically. As shown in Fig. 2.6, one starts out in mode $m$, propagates adiabatically to the scattering surface, and then couples to mode $n$, after which adiabatic propagation to the receiver in mode $n$ occurs. This process is integrated over the entire scattering surface.

If we also make the approximation that the vertical and horizontal wavenumbers in the Eq. (2.102) integrals can be replaced by their background wavenumbers, i.e. $\kappa_{n}(r) \rightarrow \kappa_{n}$ and $\gamma_{n}(r) \rightarrow \gamma_{n}$, Eq. (2.102) becomes

$$
\begin{aligned}
p_{n}^{\text {coupl }}(z, R)= & \frac{Z_{n}(z, R)}{\sqrt{\kappa_{n} R}} \sum_{m \neq n} \frac{2 i \kappa_{m} a_{n} \gamma_{n} a_{m} \gamma_{m}}{\left(\kappa_{n}^{2}-\kappa_{m}^{2}\right)} e^{i \kappa_{n} R} \\
& {\left[\int_{0}^{R} e^{i\left(\kappa_{m}-\kappa_{n}\right) r_{1}} \frac{d \xi\left(r_{1}\right)}{d r_{1}} d r_{1}\right] Z_{m}\left(z_{o}, 0\right) }
\end{aligned}
$$




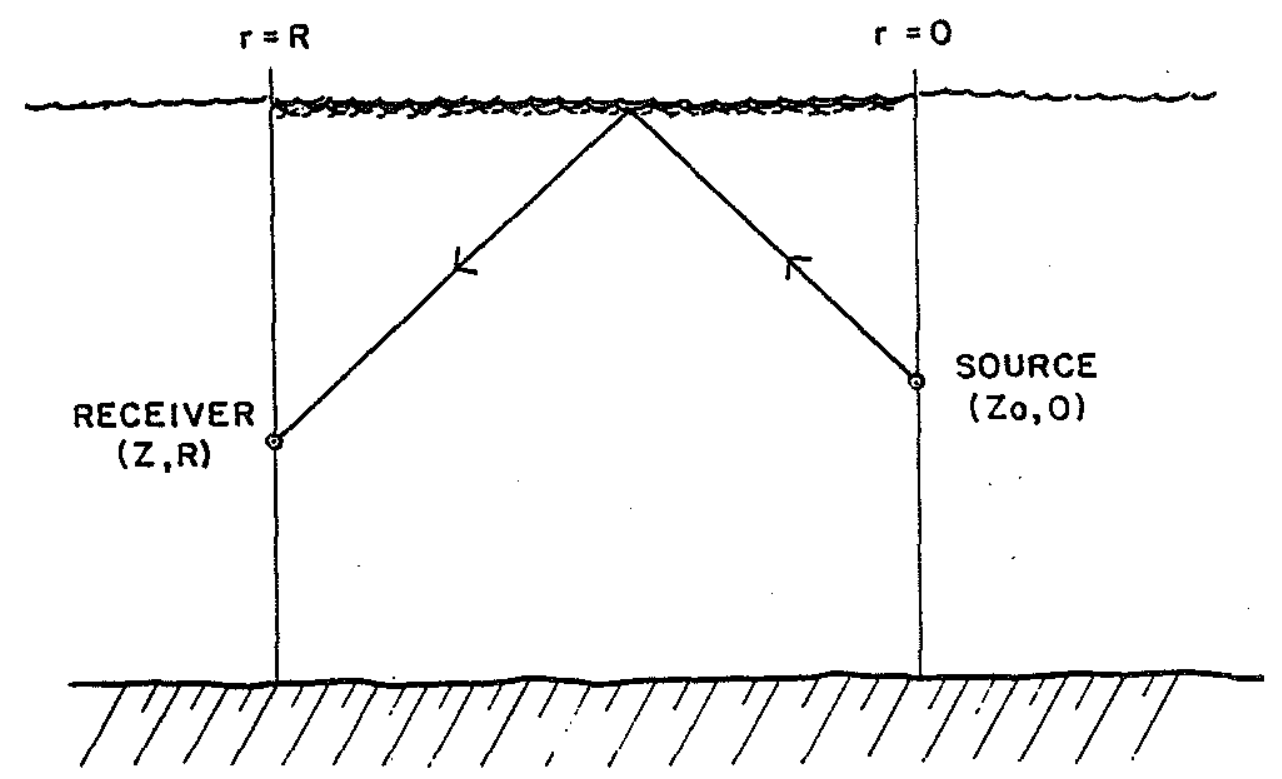

Figure 2.6: Schematic of coupled mode scattering

\subsubsection{Coupled Mode Bragg Scattering}

The single scattered coupling integral in square brackets in Eq. (2.103), which we denoted by $I$, can be written as

$$
I_{n m}=e^{i \kappa_{m} R} \int_{0}^{R} \frac{d \xi\left(r_{1}\right)}{d r_{1}} e^{-i\left(\kappa_{n}-\kappa_{m}\right) r_{1}} d r_{1} .
$$

Equation (2.104) can be interpreted as a Fourier transform (between range 0 and range $R$ ) of $\xi\left(r_{1}\right)$ evaluated at the spatial difference frequency $\left(\kappa_{n}-\kappa_{m}\right)$. As we shall see by examining the case of a sinusoidal rough surface, if the surface contributes a wavenumber to the scattered field which is just equal to the difference between modal wavenumbers, the coupled scattering is resonant, an effect commonly called "Bragg Scattering." [48].

Let us assume first that the surface height is

$$
\xi\left(r_{1}\right)=\xi_{o} \sin \left(K r_{1}\right)
$$

where $K=2 \pi / \lambda^{s}$ is the spatial wavenumber of the sinusoidal rough surface. Sub- 
stituting this into Eq. (2.104) and performing the integrations, one obtains

$$
\begin{aligned}
I_{n m}=\frac{\xi_{0} R K}{2} & \left\{\operatorname{sinc}\left[\frac{\kappa_{n}-\kappa_{m}-K}{2} R\right] e^{i\left(\frac{\kappa_{n}+\kappa_{m}+K}{2}\right) R}\right. \\
& \left.+\operatorname{sinc}\left[\frac{\kappa_{n}-\kappa_{m}+K}{2} R\right] e^{i\left(\frac{\kappa_{n}+\kappa_{m}-K}{2}\right) R}\right\} .
\end{aligned}
$$

Denoting the mode cycle distance for modes $m, n$ by

$$
\Lambda_{n m}=\left|\frac{2 \pi}{\kappa_{n}-\kappa_{m}}\right|
$$

we see that the amplitude of $I$ in Eq. (2.106) is greatest when $\Lambda_{n, m}=\lambda^{s}$ which is the Bragg scattering condition for the rough surface. When this condition is met, $I$ has an amplitude of approximately $K \xi_{0} R / 2$, i.e. the scattered pressure integral increases in direct proportion to range. Away from resonance, the $R$ in the denominator of the $\operatorname{sinc}(x)$ term comes into play giving

$$
I_{n m}=\frac{K \xi_{0}}{2\left(\frac{\kappa_{n}-\kappa_{m} \pm K}{2}\right)} \sin \left[\left(\frac{\kappa_{n}-\kappa_{m}+K}{2}\right) R\right],
$$

i.e. the range dependence is sinusoidal and always lower in amplitude than at resonance.

We can calculate the arrival time of the Bragg scattered coupled energy with a stationary phase analysis of the phase of $I$ given in Eq. (2.106). For a resonant interaction, i.e. $\kappa_{n}-\kappa_{m}= \pm K$, the phase of $I$ is just $\frac{\kappa_{n}+\kappa_{m}}{2} R$. The stationary phase method of computing arrival time involves a derivative of phase with respect to $\omega$. Therefore, the Bragg scattered energy arrives midway between the transmitted mode and the received mode arrival times.

We look at this for an ideal hard bottom waveguide example. The example waveguide is $180 \mathrm{~m}$ deep with $50 \mathrm{~Hz}$ source and receiver located $50 \mathrm{~km}$ apart, both at the bottom. There are 12 propagating modes at this frequency in this waveguide. The eigenvalues for this waveguide are listed in Table 2.9. In Fig. 2.7, 


\begin{tabular}{|c|c||}
\hline MODE NUMBER & EIGENVALUE \\
\hline 1 & 0.209258 \\
2 & 0.207797 \\
3 & 0.204844 \\
4 & 0.200333 \\
5 & 0.194156 \\
6 & 0.186146 \\
7 & 0.176053 \\
8 & 0.163494 \\
9 & 0.147839 \\
10 & 0.127958 \\
11 & 0.101394 \\
12 & 0.059827 \\
\hline
\end{tabular}

Table 2.9: Mode eigenvalues for $180 \mathrm{~m}$ ideal waveguide at $50 \mathrm{~Hz}$.

the coupled pressure magnitudes are plotted for a $.424 \mathrm{~m}$ amplitude surface wave with wavenumber $K=\kappa_{1}-\kappa_{5}$. The wavelength of the surface wave that resonates with modes 1 and 5 is about $416 \mathrm{~m}$, i.e. $\Lambda_{1,5}$, the mode cycle distance for modes 1 and 5 . The pressure amplitude values have been normalized with respect to $p_{1}^{\text {adiab }}$. Note in Fig. 2.7 that the coupled pressure given by Eq. (2.103) shows the greatest coupling into mode 1 from mode 5, where the eigenvalue difference meets the Bragg condition. Also note the amplitude of the coupled pressure is on the order of the mode 1 adiabatic pressure amplitude, calling into question the convergence of the Born series for this case. However, as we show later, a realistic sea surface spectrum scatters much less than the monochromatic sea.

We list the coupled pressures for all 12 modes for the waveguide described above in Tables 2.10 through 2.13. The mode 1-5 resonance is evident in Table 2.10. The arrival times are referenced to the arrival of the adiabatic mode 1 . The arrival time of the Bragg scattered coupled modes $(1,5)$ is midway between the arrival time of the two modes, as we calculated above. In addition, modes 8 and 9 are almost resonant 


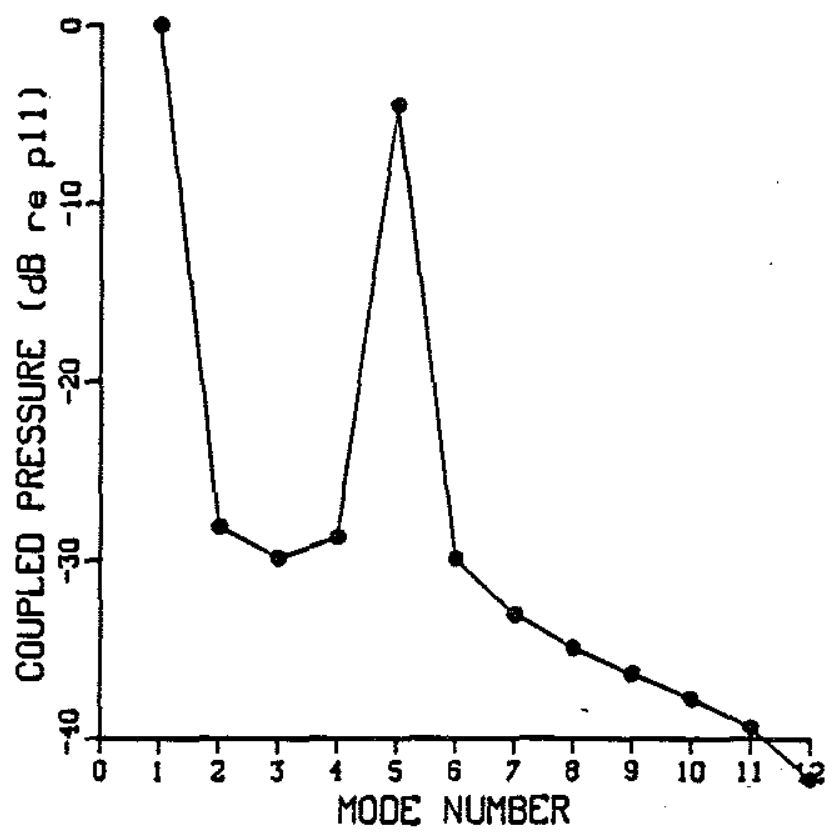

Figure 2.7: Coupled mode pressure amplitude into mode 1 for resonant Bragg scattering, for a $.424 \mathrm{~m}$ amplitude surface wave with wavenumber equal $\kappa_{1}-\kappa_{5}$. The pressure values have been normalized with respect to $p_{1}^{a d i a b}$. The ideal waveguide is $180 \mathrm{~m}$ deep with $50 \mathrm{~Hz}$ source and receiver located $50 \mathrm{~km}$ apart, both at the bottom. There are 12 propagating modes at this frequency in this waveguide. 
with the surface wave as seen in Table 2.12 because $\Lambda_{8, s}=401 \mathrm{~m}$, nearly the surface wavelength of $416 \mathrm{~m}$. The arrival times of the $(8,9)$ pair are almost midway between modes 8 and 9 . For the off resonant arrivals, the arrival time interpretations are more difficult. Moreover, the slightly negative arrival times of $(2,1)$ and $(3,1)$ are, most likely, numerical artifacts of the stationary phase calculation on very low amplitude coupled arrivals. 


\begin{tabular}{|c|c|c|c|c|}
\hline I & $\mathrm{J}$ & MAGNITUDE & PHASE (deg) & TIME (sec) \\
\hline 1 & 1 & 1.000 & 77.7 & $0.000000 \mathrm{E}+00$ \\
\hline 1 & 2 & $0.6569 \mathrm{E}-03$ & -19.1 & 0.332548 \\
\hline 1 & 3 & $0.4398 \mathrm{E}-03$ & 149.6 & 0.834960 \\
\hline 1 & 4 & $0.5778 \mathrm{E}-03$ & -40.4 & 1.19780 \\
\hline 1 & 5 & 0.1496 & 135.7 & 1.29735 \\
\hline 1 & 6 & $0.4368 \mathrm{E}-03$ & 135.9 & 1.22842 \\
\hline 1 & 7 & $0.2115 \mathrm{E}-03$ & -43.0 & 1.07142 \\
\hline 1 & 8 & $0.1372 E-03$ & 137.1 & 0.892193 \\
\hline 1 & 9 & 0.9811E-04 & -44.4 & 0.727745 \\
\hline 1 & 10 & $0.7140 \mathrm{E}-04$ & 131.9 & 0.592124 \\
\hline 1 & 11 & $0.4913 \mathrm{E}-04$ & -54.4 & 0.481649 \\
\hline 1 & 12 & $0.2551 \mathrm{E}-04$ & 113.9 & 0.389068 \\
\hline 2 & 1 & $0.6638 \mathrm{E}-03$ & -50.3 & $-.980118 \mathrm{E}-01$ \\
\hline 2 & 2 & 1.004 & -147.2 & 0.234536 \\
\hline 2 & 3 & $0.1748 \mathrm{E}-02$ & 110.2 & 0.836540 \\
\hline 2 & 4 & $0.1602 \mathrm{E}-02$ & -82.9 & 1.35511 \\
\hline 2 & 5 & $0.7566 \mathrm{E}-02$ & 91.1 & 1.52817 \\
\hline 2 & 6 & $0.1647 \mathrm{E}-02$ & 90.8 & 1.48281 \\
\hline 2 & 7 & $0.6960 \mathrm{E}-03$ & -88.0 & 1.32822 \\
\hline 2 & 8 & $0.4349 \mathrm{E}-03$ & 92.3 & 1.14409 \\
\hline 2 & 9 & $0.3060 \mathrm{E}-03$ & -89.1 & 0.976247 \\
\hline 2 & 10 & $0.2206 \mathrm{E}-03$ & 87.3 & 0.835844 \\
\hline 2 & 11 & $0.1509 \mathrm{E}-03$ & -99.0 & 0.720829 \\
\hline 2 & 12 & $0.7806 \mathrm{E}-04$ & 69.4 & 1.14194 \\
\hline 3 & 1 & $0.4540 \mathrm{E}-03$ & -38.4 & -.116120 \\
\hline 3 & 2 & $0.1786 \mathrm{E}-02$ & 136.2 & 0.116835 \\
\hline 3 & 3 & 1.011 & 33.5 & 0.718840 \\
\hline 3 & 4 & $0.3082 \mathrm{E}-02$ & -75.0 & 1.57026 \\
\hline 3 & 5 & $0.4635 \mathrm{E}-02$ & 93.5 & 1.97194 \\
\hline 3 & 6 & $0.5229 \mathrm{E}-02$ & 91.2 & 1.99872 \\
\hline 3 & 7 & $0.1448 \mathrm{E}-02$ & -87.6 & 1.86019 \\
\hline 3 & 8 & $0.8194 \mathrm{E}-03$ & 93.0 & 1.66812 \\
\hline 3 & 9 & $0.5529 \mathrm{E}-03$ & -88.1 & 1.48720 \\
\hline 3 & 10 & $0.3905 \mathrm{E}-03$ & 88.6 & 1.33894 \\
\hline 3 & 11 & $0.2640 \mathrm{E}-03$ & -97.5 & 1.21576 \\
\hline 3 & 12 & $0.1355 \mathrm{E}-03$ & 71.0 & 1.11609 \\
\hline
\end{tabular}

Table 2.10: Coupled pressure magnitude, phase and arrival time for modes 1-3. Waveguide is $180 \mathrm{~m}$ deep with a $50 \mathrm{~Hz}$ source and receiver located $50 \mathrm{~km}$ apart at the bottom. Surface wave is $.424 \mathrm{~m}$ amplitude with wavenumber equal to $\kappa_{1}-\kappa_{5}$. 


\begin{tabular}{||l|l|c|c|c||}
\hline $\mathrm{I}$ & $\mathrm{J}$ & MAGNITUDE & PHASE (deg) & TIME (sec) \\
\hline 4 & 1 & $0.6168 \mathrm{E}-03$ & -171.1 & 0.288437 \\
4 & 2 & $0.1692 \mathrm{E}-02$ & 6.5 & 0.365668 \\
4 & 3 & $0.3186 \mathrm{E}-02$ & 179.3 & -4999.37 \\
4 & 4 & 1.022 & 70.8 & 1.48624 \\
4 & 5 & $0.4940 \mathrm{E}-02$ & -42.3 & 2.51305 \\
4 & 6 & $0.3344 \mathrm{E}-01$ & 128.9 & 2.78208 \\
4 & 7 & $0.3181 \mathrm{E}-02$ & 129.1 & 2.69828 \\
4 & 8 & $0.1422 \mathrm{E}-02$ & -49.8 & 2.50117 \\
4 & 9 & $0.8858 \mathrm{E}-03$ & 129.7 & 2.29965 \\
4 & 10 & $0.6025 \mathrm{E}-03$ & -53.2 & 2.13135 \\
4 & 11 & $0.3989 \mathrm{E}-03$ & 120.9 & 2.00185 \\
4 & 12 & $0.2021 \mathrm{E}-03$ & -70.4 & 1.88894 \\
5 & 1 & 0.1673 & -44.3 & 1.29767 \\
5 & 2 & $0.8378 \mathrm{E}-02$ & 135.3 & 1.30138 \\
5 & 3 & $0.5023 \mathrm{E}-02$ & -46.4 & 1.34192 \\
5 & 4 & $0.5178 \mathrm{E}-02$ & 126.6 & 1.56821 \\
5 & 5 & 1.038 & 13.6 & 2.59502 \\
5 & 6 & $0.8052 \mathrm{E}-02$ & -103.2 & 3.75160 \\
5 & 7 & $0.1394 \mathrm{E}-01$ & -108.7 & 3.88006 \\
5 & 8 & $0.2669 \mathrm{E}-02$ & 72.6 & 3.70654 \\
5 & 9 & $0.1406 \mathrm{E}-02$ & -107.0 & 3.47967 \\
5 & 10 & $0.8946 \mathrm{E}-03$ & 70.7 & 3.28531 \\
5 & 11 & $0.5723 \mathrm{E}-03$ & -114.8 & 3.13273 \\
5 & 12 & $0.2842 \mathrm{E}-03$ & 54.2 & 3.01115 \\
6 & 1 & $0.5207 \mathrm{E}-03$ & 48.7 & 2.91386 \\
6 & 2 & $0.1943 \mathrm{E}-02$ & -131.1 & 2.89400 \\
6 & 3 & $0.6036 \mathrm{E}-02$ & 49.1 & 2.86239 \\
6 & 4 & $0.3734 \mathrm{E}-01$ & 48.7 & 2.84644 \\
6 & 5 & $0.8577 \mathrm{E}-02$ & -136.3 & 2.98569 \\
6 & 6 & 1.060 & 106.9 & 4.14227 \\
6 & 7 & $0.1399 \mathrm{E}-01$ & -12.6 & 5.37901 \\
6 & 8 & $0.7383 \mathrm{E}-02$ & -15.0 & 5.37768 \\
6 & 9 & $0.2419 \mathrm{E}-02$ & 166.4 & 5.13494 \\
6 & 10 & $0.1350 \mathrm{E}-02$ & -14.8 & 4.90071 \\
6 & 11 & $0.8149 \mathrm{E}-03$ & 160.3 & 4.71537 \\
6 & 12 & $0.3921 \mathrm{E}-03$ & -30.3 & 4.57471 \\
\hline
\end{tabular}

Table 2.11: Coupled pressure magnitude, phase and arrival time for modes 4-6. Waveguide is $180 \mathrm{~m}$ deep with a $50 \mathrm{~Hz}$ source and receiver located $50 \mathrm{~km}$ apart at the bottom. Surface wave is $.424 \mathrm{~m}$ amplitude with wavenumber equal to $\kappa_{1}-\kappa_{5}$. 


\begin{tabular}{||l|l|c|c|c||}
\hline $\mathrm{I}$ & $\mathrm{J}$ & MAGNITUDE & PHASE (deg) & TIME (sec) \\
\hline 7 & 1 & $0.2741 \mathrm{E}-03$ & 115.3 & 5.22080 \\
7 & 2 & $0.8924 \mathrm{E}-03$ & -64.6 & 5.19854 \\
7 & 3 & $0.1818 \mathrm{E}-02$ & 115.7 & 5.15088 \\
7 & 4 & $0.3861 \mathrm{E}-02$ & -63.7 & 5.08019 \\
7 & 5 & $0.1614 \mathrm{E}-01$ & 116.9 & 5.00718 \\
7 & 6 & $0.1521 \mathrm{E}-01$ & 114.0 & 5.05548 \\
7 & 7 & 1.090 & -5.4 & 6.29222 \\
7 & 8 & $0.3411 \mathrm{E}-01$ & -126.7 & 7.57678 \\
7 & 9 & $0.5471 \mathrm{E}-02$ & -126.6 & 7.44195 \\
7 & 10 & $0.2198 \mathrm{E}-02$ & 53.9 & 7.15529 \\
7 & 11 & $0.1192 \mathrm{E}-02$ & -129.8 & 6.92267 \\
7 & 12 & $0.5451 \mathrm{E}-03$ & 40.2 & 6.75378 \\
8 & 1 & $0.1987 \mathrm{E}-03$ & -46.1 & 8.44634 \\
8 & 2 & $0.6232 \mathrm{E}-03$ & 133.9 & 8.42898 \\
8 & 3 & $0.1149 \mathrm{E}-02$ & -46.2 & 8.38925 \\
8 & 4 & $0.1929 \mathrm{E}-02$ & 133.8 & 8.32360 \\
8 & 5 & $0.3454 \mathrm{E}-02$ & -45.7 & 8.22701 \\
8 & 6 & $0.8969 \mathrm{E}-02$ & 135.2 & 8.10313 \\
8 & 7 & $0.3812 \mathrm{E}-01$ & 134.6 & 8.05398 \\
8 & 8 & 1.131 & 13.3 & 9.33853 \\
8 & 9 & 0.1727 & 71.1 & 10.6353 \\
8 & 10 & $0.4442 \mathrm{E}-02$ & -107.3 & 10.3729 \\
8 & 11 & $0.1876 \mathrm{E}-02$ & 71.1 & 10.0603 \\
8 & 12 & $0.7836 \mathrm{E}-03$ & -117.8 & 9.83403 \\
9 & 1 & $0.1652 \mathrm{E}-03$ & -72.7 & 13.1325 \\
9 & 2 & $0.5098 \mathrm{E}-03$ & 107.1 & 13.1185 \\
9 & 3 & $0.9019 \mathrm{E}-03$ & -73.3 & 13.0918 \\
9 & 4 & $0.1397 \mathrm{E}-02$ & 106.2 & 13.0468 \\
9 & 5 & $0.2116 \mathrm{E}-02$ & -74.2 & 12.9756 \\
9 & 6 & $0.3418 \mathrm{E}-02$ & 105.7 & 12.8675 \\
9 & 7 & $0.7110 \mathrm{E}-02$ & -73.6 & 12.7105 \\
9 & 8 & 0.2008 & 107.3 & 12.5634 \\
9 & 9 & 1.190 & 165.2 & 13.8602 \\
9 & 10 & $0.2631 \mathrm{E}-01$ & -137.0 & 15.1291 \\
9 & 11 & $0.3524 \mathrm{E}-02$ & 44.5 & 14.7429 \\
9 & 12 & $0.1210 \mathrm{E}-02$ & -142.1 & 14.4185 \\
\hline & & & & \\
\hline & & & \\
\hline
\end{tabular}

Table 2.12: Coupled pressure magnitude, phase and arrival time for modes 7-9. Waveguide is $180 \mathrm{~m}$ deep with a $50 \mathrm{~Hz}$ source and receiver located $50 \mathrm{~km}$ apart at the bottom. Surface wave is $.424 \mathrm{~m}$ amplitude with wavenumber equal to $\kappa_{1}-\kappa_{5}$. 
The actual ocean surface is much more complicated than a single sinusoidal wave propagating in one direction. A realistic sea surface is made up of a sum of many different frequency sinusoidal waves travelling in different directions, each with a random phase. This sum is called the frequency-direction spectrum $F(\Omega, \alpha)$ where $\Omega$ is the radian frequency of the wave and $\alpha$ is the direction the wave is travelling. The wavenumber of the surface wave $K$ is related to the frequency $\Omega$ by the appropriate dispersion relation. Here, we assume deep water waves with $\Omega^{2}=$ $g K$. The frequency-direction spectrum is discussed in greater detail in Chapter 3.

Here, as another example, we show the effect of coupling on a more complicated surface made up of four sinusoids or

$$
\xi\left(r_{1}\right)=\sum_{i=1}^{4} \xi_{i} \sin \left(K_{i} r_{1}+\phi_{i}\right)
$$

where $\phi_{i}$ are random phases and we will assume that all $\xi_{i}=.15$ so that the rms roughness of the sea surface remains that for the previous example. Now, we assume that

$$
K_{i}=k_{1+i}-k_{5+i}
$$

We list the coupled pressures for all 12 modes for the waveguide under the more complicated sea in Tables 2.14 through 2.17. The mode 1-5, 2-6, and 3-7 resonances are evident in Table 2.14. The mode 4-8 resonance is shown in Table 2.15. Because the sea surface energy (variance) is now spread between four waves, the coupling amplitude is generally lower as we note for the 1-5 resonance in Table 2.14. All the resonant pairs, (1-5), 2-6), (3-7), and (4-8), have high coupled energy and arrive at times about halfway between the sender and reciever mode arrival times. However, the coupling amplitude at higher mode numbers is greater than that at lower mode number. The effect is caused by the higher vertical eigenvalues at higher mode number in Eq. 2.103. A more physical interpretation of this is that the equivalent 


\begin{tabular}{||l|l|c|c|c||}
\hline $\mathrm{I}$ & $\mathrm{J}$ & MAGNITUDE & PHASE (deg) & TIME (sec) \\
\hline 10 & 1 & $0.1493 \mathrm{E}-03$ & 35.8 & 20.6051 \\
10 & 2 & $0.4564 \mathrm{E}-03$ & -144.5 & 20.5959 \\
10 & 3 & $0.7910 \mathrm{E}-03$ & 34.9 & 20.5771 \\
10 & 4 & $0.1180 \mathrm{E}-02$ & -146.0 & 20.5521 \\
10 & 5 & $0.1672 \mathrm{E}-02$ & 32.9 & 20.5070 \\
10 & 6 & $0.2368 \mathrm{E}-02$ & -148.3 & 20.4388 \\
10 & 7 & $0.3547 \mathrm{E}-02$ & 30.7 & 20.3342 \\
10 & 8 & $0.6416 \mathrm{E}-02$ & -149.4 & 20.1628 \\
10 & 9 & $0.3267 \mathrm{E}-01$ & 32.2 & 19.9284 \\
10 & 10 & 1.279 & 90.0 & 21.1972 \\
10 & 11 & $0.1302 \mathrm{E}-01$ & 148.6 & 22.3749 \\
10 & 12 & $0.2180 \mathrm{E}-02$ & -33.2 & 21.8726 \\
11 & 1 & $0.1457 \mathrm{E}-03$ & 83.4 & 35.0091 \\
11 & 2 & $0.4426 \mathrm{E}-03$ & -96.9 & 35.0044 \\
11 & 3 & $0.7580 \mathrm{E}-03$ & 82.3 & 34.9938 \\
11 & 4 & $0.1108 \mathrm{E}-02$ & -98.8 & 34.9751 \\
11 & 5 & $0.1516 \mathrm{E}-02$ & 79.7 & 34.9530 \\
11 & 6 & $0.2027 \mathrm{E}-02$ & -102.2 & 34.9176 \\
11 & 7 & $0.2727 \mathrm{E}-02$ & 75.7 & 34.8603 \\
11 & 8 & $0.3840 \mathrm{E}-02$ & -106.5 & 34.7689 \\
11 & 9 & $0.6204 \mathrm{E}-02$ & 71.9 & 34.6081 \\
11 & 10 & $0.1845 \mathrm{E}-01$ & -107.3 & 34.3131 \\
11 & 11 & 1.437 & -48.7 & 35.4907 \\
11 & 12 & $0.6215 \mathrm{E}-02$ & 10.8 & 36.4366 \\
12 & 1 & $0.1669 \mathrm{E}-03$ & -9.0 & 82.9406 \\
12 & 2 & $0.5053 \mathrm{E}-03$ & 170.6 & 82.4223 \\
12 & 3 & $0.8585 \mathrm{E}-03$ & -10.3 & 82.9324 \\
12 & 4 & $0.1238 \mathrm{E}-02$ & 168.4 & 82.9270 \\
12 & 5 & $0.1661 \mathrm{E}-02$ & -13.4 & 82.9136 \\
12 & 6 & $0.2152 \mathrm{E}-02$ & 164.3 & 82.8973 \\
12 & 7 & $0.2752 \mathrm{E}-02$ & -18.4 & 82.8681 \\
12 & 8 & $0.3540 \mathrm{E}-02$ & 158.3 & 82.8342 \\
12 & 9 & $0.4701 \mathrm{E}-02$ & -25.6 & 82.7713 \\
12 & 10 & $0.6819 \mathrm{E}-02$ & 150.4 & 82.6544 \\
12 & 11 & $0.1371 \mathrm{E}-01$ & -32.4 & 82.3838 \\
12 & 12 & 1.870 & 27.2 & 83.3297 \\
\hline
\end{tabular}

Table 2.13: Coupled pressure magnitude, phase and arrival time for modes 10-12. Waveguide is $180 \mathrm{~m}$ deep with a $50 \mathrm{~Hz}$ source and receiver located $50 \mathrm{~km}$ apart at the bottom. Surface wave is $.424 \mathrm{~m}$ amplitude with wavenumber equal to $\kappa_{1}-\kappa_{5}$. 
rays for these higher modes are bouncing off the rough surface more often per unit range, and therefore, the coupling into other modes is higher. 


\begin{tabular}{||l|l|c|c|c||}
\hline $\mathrm{I}$ & $\mathrm{J}$ & MAGNITUDE & PHASE (deg) & TIME (sec) \\
\hline 1 & 1 & 1.000 & 77.7 & $0.000000 \mathrm{E}+00$ \\
1 & 2 & $0.4170 \mathrm{E}-03$ & 172.3 & $0.481027 \mathrm{E}-01$ \\
1 & 3 & $0.2387 \mathrm{E}-03$ & 1.9 & 0.117424 \\
1 & 4 & $0.2007 \mathrm{E}-03$ & -154.3 & $0.647988 \mathrm{E}-01$ \\
1 & 5 & $0.5264 \mathrm{E}-01$ & 112.7 & 1.30291 \\
1 & 6 & $0.1197 \mathrm{E}-02$ & -12.6 & 1.96671 \\
1 & 7 & $0.7353 \mathrm{E}-03$ & -167.7 & 3.26363 \\
1 & 8 & $0.7704 \mathrm{E}-04$ & -140.1 & 6.31447 \\
1 & 9 & $0.1468 \mathrm{E}-04$ & 66.0 & 10.5392 \\
1 & 10 & $0.5777 \mathrm{E}-05$ & -95.4 & 12.6828 \\
1 & 11 & $0.2772 \mathrm{E}-05$ & 95.7 & 12.6077 \\
1 & 12 & $0.1103 \mathrm{E}-05$ & -79.0 & 11.0153 \\
2 & 1 & $0.4214 \mathrm{E}-03$ & 118.3 & 0.186433 \\
2 & 2 & 1.004 & -147.2 & 0.234536 \\
2 & 3 & $0.1047 \mathrm{E}-02$ & -48.3 & 0.321410 \\
2 & 4 & $0.6578 \mathrm{E}-03$ & 150.7 & 0.346191 \\
2 & 5 & $0.2094 \mathrm{E}-02$ & 45.7 & 0.978093 \\
2 & 6 & 0.1744 & 116.8 & 2.19323 \\
2 & 7 & $0.2284 \mathrm{E}-02$ & 144.0 & 3.30643 \\
2 & 8 & $0.3054 \mathrm{E}-03$ & 172.2 & 6.13228 \\
2 & 9 & $0.4938 \mathrm{E}-04$ & 19.1 & 10.4594 \\
2 & 10 & $0.1841 \mathrm{E}-04$ & -141.3 & 12.8353 \\
2 & 11 & $0.8627 \mathrm{E}-05$ & 50.5 & 12.8781 \\
2 & 12 & $0.3396 \mathrm{E}-05$ & -124.0 & 11.2454 \\
3 & 1 & $0.2465 \mathrm{E}-03$ & 109.4 & 0.601415 \\
3 & 2 & $0.1070 \mathrm{E}-02$ & -65.4 & 0.631965 \\
3 & 3 & 1.011 & 33.5 & 0.718840 \\
3 & 4 & $0.1657 \mathrm{E}-02$ & 138.1 & 0.838206 \\
3 & 5 & $0.1328 \mathrm{E}-02$ & -1.4 & 0.744470 \\
3 & 6 & $0.5788 \mathrm{E}-02$ & 103.2 & 2.58104 \\
3 & 7 & 0.3802 & -43.5 & 3.51100 \\
3 & 8 & $0.1111 \mathrm{E}-02$ & 166.5 & 5.75284 \\
3 & 9 & $0.1052 \mathrm{E}-03$ & 15.6 & 10.2817 \\
3 & 10 & $0.3476 \mathrm{E}-04$ & -142.7 & 13.1819 \\
3 & 11 & $0.1555 \mathrm{E}-04$ & 50.3 & 13.4457 \\
3 & 12 & $0.5979 \mathrm{E}-05$ & -123.4 & 11.9344 \\
\hline
\end{tabular}

Table 2.14: Coupled pressure magnitude, phase and arrival time for modes 1-3. Waveguide is $180 \mathrm{~m}$ deep with a $50 \mathrm{~Hz}$ source and receiver located $50 \mathrm{~km}$ apart at the bottom. Surface has four sinusoidal components. 


\begin{tabular}{||l|l|c|c|c||}
\hline $\mathrm{I}$ & $\mathrm{J}$ & MAGNITUDE & PHASE (deg) & TIME (sec) \\
\hline 4 & 1 & $0.2142 \mathrm{E}-03$ & -57.2 & 1.42144 \\
4 & 2 & $0.6949 \mathrm{E}-03$ & 132.9 & 1.37459 \\
4 & 3 & $0.1713 \mathrm{E}-02$ & -33.9 & 1.36687 \\
4 & 4 & 1.022 & 70.8 & 1.48624 \\
4 & 5 & $0.2304 \mathrm{E}-02$ & -177.8 & 1.61374 \\
4 & 6 & $0.9923 \mathrm{E}-02$ & 92.2 & 2.45061 \\
4 & 7 & $0.4678 \mathrm{E}-02$ & -15.5 & 3.39743 \\
4 & 8 & 0.5928 & -166.2 & 5.41437 \\
4 & 9 & $0.2335 \mathrm{E}-03$ & -134.4 & 9.84298 \\
4 & 10 & $0.6026 \mathrm{E}-04$ & 70.8 & 13.5888 \\
4 & 11 & $0.2470 \mathrm{E}-04$ & -93.9 & 14.2813 \\
4 & 12 & $0.9121 \mathrm{E}-05$ & 93.6 & 12.8461 \\
5 & 1 & $0.5890 \mathrm{E}-01$ & -21.4 & 1.29211 \\
5 & 2 & $0.2319 \mathrm{E}-02$ & -179.2 & 1.85146 \\
5 & 3 & $0.1440 \mathrm{E}-02$ & 48.5 & 2.56939 \\
5 & 4 & $0.2415 \mathrm{E}-02$ & -97.8 & 2.46752 \\
5 & 5 & 1.038 & 13.6 & 2.59502 \\
5 & 6 & $0.3088 \mathrm{E}-02$ & 135.3 & 2.69027 \\
5 & 7 & $0.1201 \mathrm{E}-01$ & -100.8 & 4.41831 \\
5 & 8 & $0.1002 \mathrm{E}-01$ & -58.1 & 5.53758 \\
5 & 9 & $0.7207 \mathrm{E}-03$ & -23.0 & 9.10100 \\
5 & 10 & $0.1093 \mathrm{E}-03$ & -172.4 & 13.9640 \\
5 & 11 & $0.3831 \mathrm{E}-04$ & 26.5 & 15.4455 \\
5 & 12 & $0.1325 \mathrm{E}-04$ & -144.1 & 14.2423 \\
6 & 1 & $0.1427 \mathrm{E}-02$ & -162.8 & 2.17556 \\
6 & 2 & 0.2057 & -157.1 & 2.18358 \\
6 & 3 & $0.6682 \mathrm{E}-02$ & 37.1 & 2.28007 \\
6 & 4 & $0.1108 \mathrm{E}-01$ & 85.5 & 3.17791 \\
6 & 5 & $0.3289 \mathrm{E}-02$ & -14.8 & 4.04703 \\
6 & 6 & 1.060 & 106.9 & 4.14227 \\
6 & 7 & $0.4280 \mathrm{E}-02$ & -115.0 & 4.17085 \\
6 & 8 & $0.3070 \mathrm{E}-01$ & -165.3 & 6.11176 \\
6 & 9 & $0.1204 \mathrm{E}-01$ & -126.7 & 8.40761 \\
6 & 10 & $0.2400 \mathrm{E}-03$ & 90.6 & 13.9814 \\
6 & 11 & $0.6186 \mathrm{E}-04$ & -64.2 & 16.9298 \\
6 & 12 & $0.1919 \mathrm{E}-04$ & 128.3 & 16.1195 \\
\hline & & & \\
\hline
\end{tabular}

Table 2.15: Coupled pressure magnitude, phase and arrival time for modes 4-6. Waveguide is $180 \mathrm{~m}$ deep with a $50 \mathrm{~Hz}$ source and receiver located $50 \mathrm{~km}$ apart at the bottom. Surface has four sinusoidal components. 


\begin{tabular}{||l|l|c|c|c||}
\hline $\mathrm{I}$ & $\mathrm{J}$ & MAGNITUDE & PHASE (deg) & TIME (sec) \\
\hline 7 & 1 & $0.9528 \mathrm{E}-03$ & -120.0 & 3.02860 \\
7 & 2 & $0.2929 \mathrm{E}-02$ & 63.4 & 3.22032 \\
7 & 3 & 0.4772 & 71.6 & 3.50006 \\
7 & 4 & $0.5678 \mathrm{E}-02$ & 80.9 & 4.38104 \\
7 & 5 & $0.1391 \mathrm{E}-01$ & 109.0 & 4.46893 \\
7 & 6 & $0.4653 \mathrm{E}-02$ & -143.5 & 6.26364 \\
7 & 7 & 1.090 & -5.4 & 6.29222 \\
7 & 8 & $0.8817 \mathrm{E}-02$ & 167.6 & 6.59116 \\
7 & 9 & $0.2910 \mathrm{E}-01$ & -84.1 & 9.05431 \\
7 & 10 & $0.8979 \mathrm{E}-03$ & 141.4 & 13.3362 \\
7 & 11 & $0.1121 \mathrm{E}-03$ & -3.4 & 18.5965 \\
7 & 12 & $0.2864 \mathrm{E}-04$ & -165.5 & 18.6037 \\
8 & 1 & $0.1116 \mathrm{E}-03$ & -128.8 & 3.02406 \\
8 & 2 & $0.4376 \mathrm{E}-03$ & 54.0 & 3.44079 \\
8 & 3 & $0.1558 \mathrm{E}-02$ & -119.7 & 4.30454 \\
8 & 4 & 0.8040 & -109.7 & 5.41040 \\
8 & 5 & $0.1297 \mathrm{E}-01$ & 85.0 & 6.39597 \\
8 & 6 & $0.3729 \mathrm{E}-01$ & -74.5 & 7.36904 \\
8 & 7 & $0.9852 \mathrm{E}-02$ & -159.6 & 9.03960 \\
8 & 8 & 1.131 & 13.3 & 9.33853 \\
8 & 9 & $0.7076 \mathrm{E}-01$ & 56.6 & 10.8237 \\
8 & 10 & $0.3278 \mathrm{E}-01$ & 133.4 & 13.0127 \\
8 & 11 & $0.2725 \mathrm{E}-03$ & -177.6 & 20.0029 \\
8 & 12 & $0.4605 \mathrm{E}-04$ & 30.2 & 22.0719 \\
9 & 1 & $0.2473 \mathrm{E}-04$ & 176.9 & 3.32098 \\
9 & 2 & $0.8229 \mathrm{E}-04$ & -1.1 & 3.63531 \\
9 & 3 & $0.1716 \mathrm{E}-03$ & -176.9 & 4.29732 \\
9 & 4 & $0.3683 \mathrm{E}-03$ & 10.4 & 5.50346 \\
9 & 5 & $0.1085 \mathrm{E}-02$ & -158.2 & 7.35423 \\
9 & 6 & $0.1701 \mathrm{E}-01$ & 38.8 & 9.59486 \\
9 & 7 & $0.3782 \mathrm{E}-01$ & -116.1 & 11.0981 \\
9 & 8 & $0.8229 \mathrm{E}-01$ & 121.9 & 12.3751 \\
9 & 9 & 1.190 & 165.2 & 13.8602 \\
9 & 10 & $0.5186 \mathrm{E}-01$ & -118.8 & 15.7719 \\
9 & 11 & $0.1775 \mathrm{E}-02$ & 128.8 & 20.4023 \\
9 & 12 & $0.8697 \mathrm{E}-04$ & -4.2 & 26.7099 \\
\hline & & & & \\
\hline
\end{tabular}

Table 2.16: Coupled pressure magnitude, phase and arrival time for modes 7-9. Waveguide is $180 \mathrm{~m}$ deep with a $50 \mathrm{~Hz}$ source and receiver located $50 \mathrm{~km}$ apart at the bottom. Surface wave has four sinusoidal components. 


\subsubsection{Coupling Strength}

To roughly estimate the importance of coupling (or, in other words, the convergence properties of the Born series for the Bragg scattering by the surface), we compute the ratio of amplitudes of typical coupled pressure terms to the adiabatic pressure

$$
S_{n m}=\frac{\left|p_{n m}^{\text {coupl }}(z, R)\right|}{\left|p_{n}^{\text {diab }}(z, R)\right|}=a_{m} \gamma_{m} a_{n} \gamma_{n} \frac{\kappa_{m}}{\kappa_{n}^{2}-\kappa_{m}^{2}} \xi_{0} K R\left|\frac{Z_{m}\left(z_{0}, 0\right)}{Z_{n}\left(z_{0}, 0\right)}\right|
$$

Of course, this ignores any phase interference effect but the order of magnitude of the ratio is indicative of the coupling strengths. Since $K=\kappa_{n}-\kappa_{m}$ at the Bragg condition, and $a_{n} \sim \sqrt{2 /(\rho H)}$, the ratio becomes

$$
S_{n m}=\frac{\left|p_{n m}^{\text {coupl }}(z, R)\right|}{\left|p_{n}^{\text {adiab }}(z, R)\right|}=\frac{2 \gamma_{m} \gamma_{n}}{\rho H} \frac{\kappa_{m}}{\kappa_{n}+\kappa_{m}} \xi_{0} R\left|\frac{Z_{m}\left(z_{0}, 0\right)}{Z_{n}\left(z_{0}, 0\right)}\right|
$$

The series converges best for low mode numbers and deep waveguides as we would expect. Also, as range $R$ increases, the coupling increases giving a range limit to the adiabatic approximation. One also sees that modes which start with little adiabatic energy, e.g. with a null of the mode near the source depth, are very susceptable to coupling from other modes.

We can calculate $S_{n m}$ for our example wavguide from the previous section, with a depth of $180 \mathrm{~m}, 50 \mathrm{~Hz}$ source with a receiver located $50 \mathrm{~km}$. The ratios depend on the depth of the source and reciever. We assume source and receiver depths are the same. The sea surface amplitude is $\xi_{0}=.424 \mathrm{~m}$. The results are shown in Table 2.18 for the $S_{1 m}$ terms, and agree qualitatively well with the explicitly calculated results from the previous section. 


\begin{tabular}{||l|l|c|c|c||}
\hline $\mathrm{I}$ & $\mathrm{J}$ & MAGNITUDE & PHASE (deg) & TIME (sec) \\
\hline 10 & 1 & $0.1208 \mathrm{E}-04$ & -96.8 & 8.51440 \\
10 & 2 & $0.3809 \mathrm{E}-04$ & 84.2 & 8.59645 \\
10 & 3 & $0.7040 \mathrm{E}-04$ & -93.8 & 8.73416 \\
10 & 4 & $0.1180 \mathrm{E}-03$ & 89.9 & 9.09472 \\
10 & 5 & $0.2043 \mathrm{E}-03$ & -84.0 & 9.82822 \\
10 & 6 & $0.4210 \mathrm{E}-03$ & 106.2 & 11.3581 \\
10 & 7 & $0.1449 \mathrm{E}-02$ & -56.8 & 14.1533 \\
10 & 8 & $0.4734 \mathrm{E}-01$ & -30.1 & 17.5231 \\
10 & 9 & $0.6440 \mathrm{E}-01$ & 14.0 & 19.2856 \\
10 & 10 & 1.279 & 90.0 & 21.1972 \\
10 & 11 & $0.1612 \mathrm{E}-02$ & 31.6 & 1.89469 \\
10 & 12 & $0.2490 \mathrm{E}-03$ & 86.0 & 32.8346 \\
11 & 1 & $0.8220 \mathrm{E}-05$ & -66.7 & 22.8830 \\
11 & 2 & $0.2531 \mathrm{E}-04$ & 113.6 & 22.8472 \\
11 & 3 & $0.4466 \mathrm{E}-04$ & -65.5 & 22.7638 \\
11 & 4 & $0.6859 \mathrm{E}-04$ & 116.0 & 22.6956 \\
11 & 5 & $0.1015 \mathrm{E}-03$ & -61.6 & 22.6403 \\
11 & 6 & $0.1539 \mathrm{E}-03$ & 122.4 & 22.7032 \\
11 & 7 & $0.2566 \mathrm{E}-03$ & -50.7 & 23.1865 \\
11 & 8 & $0.5579 \mathrm{E}-03$ & 142.2 & 24.8264 \\
11 & 9 & $0.3125 \mathrm{E}-02$ & -12.4 & 28.9487 \\
11 & 10 & $0.2286 \mathrm{E}-02$ & 9.7 & 54.7933 \\
11 & 11 & 1.437 & -48.7 & 35.4907 \\
11 & 12 & $0.7874 \mathrm{E}-02$ & 84.8 & 40.5937 \\
12 & 1 & $0.7215 \mathrm{E}-05$ & -176.1 & 72.3144 \\
12 & 2 & $0.2198 \mathrm{E}-04$ & 4.0 & 72.3188 \\
12 & 3 & $0.3788 \mathrm{E}-04$ & -175.9 & 72.1141 \\
12 & 4 & $0.5589 \mathrm{E}-04$ & 4.4 & 71.9698 \\
12 & 5 & $0.7749 \mathrm{E}-04$ & -175.2 & 71.6824 \\
12 & 6 & $0.1053 \mathrm{E}-03$ & 5.7 & 71.3525 \\
12 & 7 & $0.1446 \mathrm{E}-03$ & -172.7 & 71.0182 \\
12 & 8 & $0.2081 \mathrm{E}-03$ & 10.3 & 70.5963 \\
12 & 9 & $0.3378 \mathrm{E}-03$ & -163.5 & 70.4800 \\
12 & 10 & $0.7789 \mathrm{E}-03$ & 31.2 & 71.6923 \\
12 & 11 & $0.1737 \mathrm{E}-01$ & -106.3 & 78.2268 \\
12 & 12 & 1.870 & 27.2 & 83.3297 \\
\hline
\end{tabular}

Table 2.17: Coupled pressure magnitude, phase and arrival time for modes 10-12. Waveguide is $180 \mathrm{~m}$ deep with a $50 \mathrm{~Hz}$ source and receiver located $50 \mathrm{~km}$ apart at the bottom. Surface has four sinusoidal components. 


\begin{tabular}{||c|l|}
\hline $\mathrm{m}$ & $S_{1 \mathrm{n}}$ \\
\hline 2 & 0.026814 \\
3 & 0.044369 \\
4 & 0.061417 \\
5 & 0.077702 \\
6 & 0.092895 \\
7 & 0.106553 \\
8 & 0.118021 \\
9 & 0.126252 \\
10 & 0.129330 \\
11 & 0.122955 \\
12 & 0.091733 \\
\hline
\end{tabular}

Table 2.18: Coupling strengths into mode 1 for $180 \mathrm{~m}$ waveguide, frequency of $50 \mathrm{~Hz}$, and range of $50 \mathrm{~km}$.

\subsubsection{WKB Approximation Breakdown}

An error in the WKB approximation can limit the accuracy of our method. We can write the phase of a mode with coupling as

$$
\varphi_{m}(R)=\varphi_{m}^{(0)}(R)+\varphi_{m}^{(2)}(R)
$$

where

$$
\varphi_{m}^{(0)}=\int_{0}^{R} \kappa_{m}\left(r^{\prime}\right) d r^{\prime}
$$

is the zeroth order perturbation to the phase, what we described as the WKB approximation. There is no first order perturbation. The second order phase perturbation due to coupling is given by $[52,53]$

$$
\varphi_{m}^{(2)}=\frac{1}{2} \int_{0}^{R} \sum_{n \neq m} \frac{B_{n m}^{2}\left(r_{1}\right) \kappa_{m}\left(r_{1}\right)}{\kappa_{m}^{2}-\kappa_{n}^{2}} d r_{1}
$$

and using the same assumptions as above, Eq. (2.115) becomes

$$
\varphi_{m}^{(2)}=2 \int_{0}^{R}\left(\frac{\partial \xi}{\partial r_{1}}\right)^{2} d r_{1} \sum_{n \neq m} \frac{a_{m}^{2} \gamma_{m}^{2} a_{n}^{2} \gamma_{n}^{2} \kappa_{m}}{\left(\kappa_{m}^{2}-\kappa_{n}^{2}\right)^{3}}
$$


For the WKB approximation to be valid, $\varphi_{m}^{(2)} \ll \pi$. We make the approximation that

$$
\int_{0}^{R}\left(\frac{\partial \xi}{\partial r_{1}}\right)^{2} d r_{1} \simeq R \sigma_{\dot{\xi}}^{2}
$$

where $\sigma_{\dot{\xi}}$ is the rms slope of the sea surface. Thus we get that

$$
\varphi_{m}^{(2)} \simeq 2 R \sigma_{\dot{\xi}}^{2} \sum_{n \neq m} \frac{a_{m}^{2} \gamma_{m}^{2} a_{n}^{2} \gamma_{n}^{2} \kappa_{m}}{\left(\kappa_{m}^{2}-\kappa_{n}^{2}\right)^{3}}
$$

Evaluating the above expression for the ideal hard-bottom waveguide shown in Fig. 2.4 for depths greater than $30 \mathrm{~m}$, for a frequency of $220 \mathrm{~Hz}$, and at a range less than $500 \mathrm{~km}, \varphi_{m}^{(2)}<10^{-2}$ for all modes. Therefore, the WKB mode phase approximation is valid for our application. This result is not surprising because this error is second-order in the ratio of sea surface height to depth.

\subsection{Rays}

In Section 2.2.1, we review the equations for ray acoustics for a three dimensionally varying ocean. We review the derivation for the eikonal equation, which governs the path geometry of the rays. We also review the transport equation which determines ray amplitude. These are the standard textbook derivations which may be safely ignored by the reader familiar with them. Next, in Section 2.2.2, we look at the perturbations to the ray travel time and phase fluctuations due to a rough sea surface. The phase and travel time covariances are derived as functions of the sea surface covariance, as we did for the modes. 


\subsubsection{Eikonal and Transport Equations}

Ray theory[54] also begins with the Helmholtz Eq. (2.3), which we rewrite here for sound pressure $p(\vec{r})$ at location $\vec{r}$ as

$$
\nabla^{2} p+k^{2}(\vec{r}) p=0
$$

where

$$
k(\vec{r})=\frac{\omega}{c(\vec{r})}=k_{0} n(\vec{r}),
$$

$k_{0}=\omega / c_{0}, c_{0}$ being the sound speed at some reference point and $n(\vec{r})$ is the index of refraction. We represent the sound pressure as

$$
p(\vec{r})=A(\vec{r}) e^{i k_{0} W(\vec{r})}
$$

where $A$ is the amplitude of the wave, $k_{0} W$ is the phase of the ray, and $W$ is referred to as the eikonal. Substitution of Eq. (2.121) into the Helmholtz Eq. (2.119) yields

$$
\nabla^{2} A+i k_{0}\left(2 \nabla A \cdot \nabla W+A \nabla^{2} W\right)+k_{0}^{2} A\left[n^{2}-(\nabla W)^{2}\right]=0 .
$$

The equations for ray theory are obtained from Eq. (2.122) when $k_{0} \rightarrow \infty$ (the sound wavelength $\lambda=2 \pi / k_{0} \rightarrow 0$ ). Neglecting the first term in Eq. (2.122) and then equating the real and imaginary parts separately to zero, we obtain two equations: the eikonal equation

$$
(\nabla W)^{2}=n^{2}
$$

and the transport equation

$$
2 \nabla A \cdot \nabla W+A \nabla^{2} W=0
$$

The eikonal equation (2.123) defines the geometry of the rays, i.e. the lines orthogonal to the wave fronts, with $W=$ const. The transport equation determines the amplitude. 
We will be mainly interested in the eikonal equation, from which we get the phase and travel time of eigenrays. Eigenrays are defined as those ray paths starting from the source that intersect the receiver location[40]. The travel time of the $\mathrm{m}^{\text {th }}$ eigenray is given by

$$
\tau_{m}^{\mathrm{ray}}=\frac{1}{c_{0}} \int_{S_{m}} n d s
$$

where $S_{m}$ defines the $\mathrm{m}^{\text {th }}$ eigenray path. The phase of the $\mathrm{m}^{\text {th }}$ eigenray is given by

$$
\varphi_{m}^{\mathrm{ray}}=\omega \tau_{m}^{\mathrm{ray}}=k_{0} \int_{S_{m}} n d s
$$

These quantities can be computed using numerical ray trace programs[55].

\subsubsection{Scattering of Rays from a Rough Surface}

As shown in Fig. 2.8, a ray impinging on a rough surface will have two major differences from a ray impinging on a flat surface: 1) the ray will have an phase shift due to the surface elevation change, and 2) its direction will change according to the law of reflection. We can write the $n^{\text {th }}$ ray's phase variation due to a surface displacement $\xi\left(r_{1}, t_{1}\right)$ as

$$
\Delta \varphi_{n}\left(t_{1}\right)=\left(2 k_{0} \sin \theta_{n}\right) \sum_{k} \xi\left(r_{n k}, t_{1}\right)
$$

where $\theta_{n}$ is the ray's angle of incidence, $k_{0}$ is the acoustic wavenumber at the surface, and we again assume a "frozen" surface during the time-of-flight of the ray. The covariance of the phase variation in Eq. (2.127) is

$$
\vartheta_{n}^{\mathrm{ray}}(t)=\left(2 k_{0} \sin \theta_{n}\right)^{2} \sum_{l} \sum_{m} z\left(r_{l m}, t\right)
$$

where now $r_{l m}=r_{n l}-r_{n m}=(l-m) \Delta r, t=t_{1}-t_{2}, Z(r, t)$ is again the space-time covariance function of the sea surface, and $\Delta r$ is the ray skip distance. Since the 


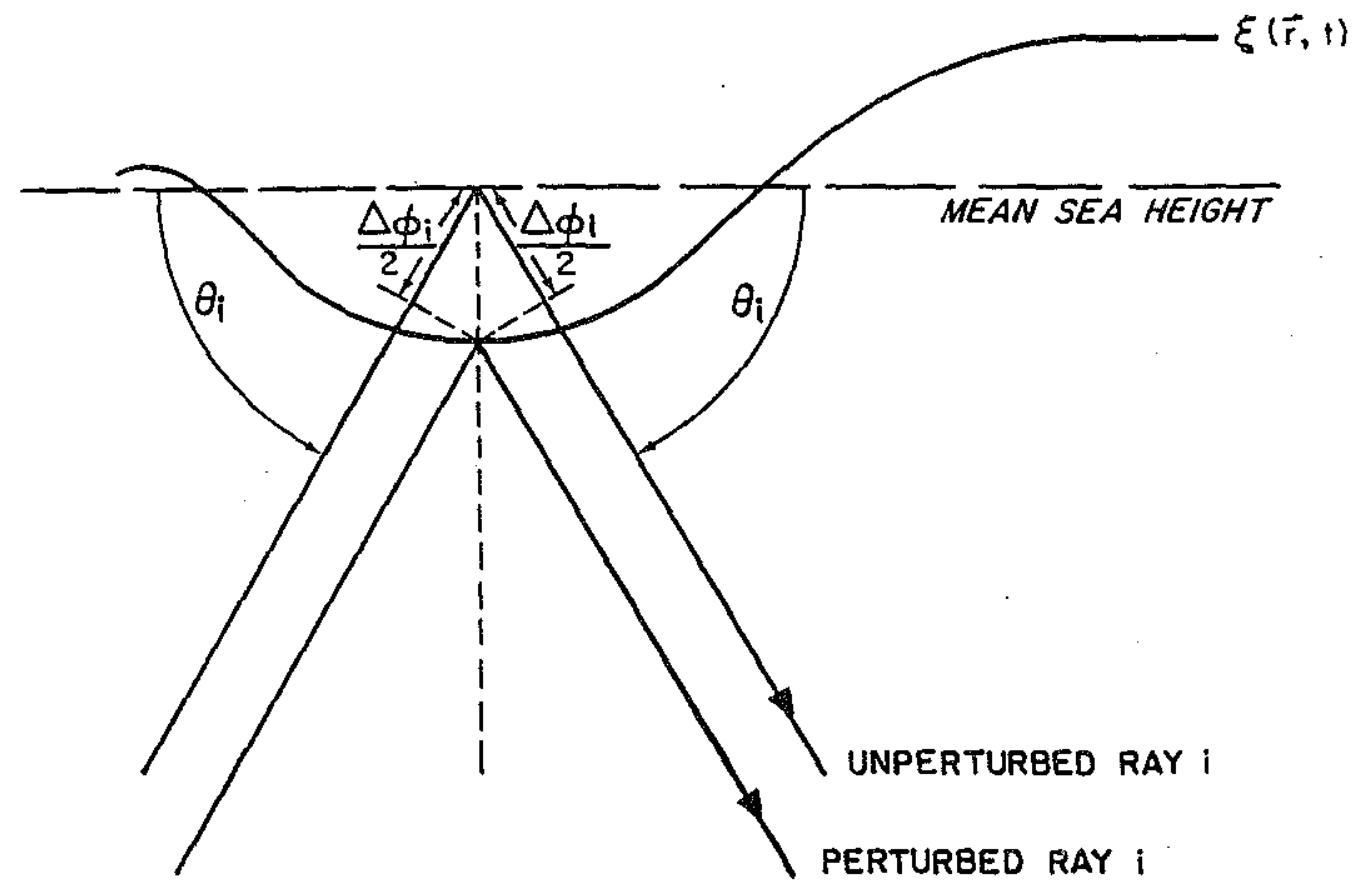

Figure 2.8: Ray reflected from rough surface

double sum in Eq. (2.128) depends only on the difference between $l$ and $m$, we can rewrite Eq. (2.128) as

$$
\vartheta_{n}^{\text {ray }}(t)=\left(2 k_{0} \sin \theta_{n}\right)^{2} \sum_{m=-M}^{+M}(M-|m|) Z\left(r_{m}, t\right)
$$

where $r_{m}=m \Delta r$.

The travel time and phase of a ray were shown above to be related by $\varphi=\omega \tau$ so the ray travel time fluctuation spectra can be written in an analogous manner to the phase, i.e.

$$
v_{n}^{\mathrm{ray}}(t)=\left(2 c_{0}^{-1} \sin \theta_{n}\right)^{2} \sum_{m=-M}^{+M}(M-|m|) Z\left(r_{m}, t\right)
$$

where $c_{0}$ is the sound speed at the sea surface.

\subsection{Summary of Scattering Results}

For both modes and rays, we have related the covariance of the measured arrival phase and travel time to the covariance of the sea surface. The equations below 
summarize our findings.

Mode Phase Covariance

$$
\vartheta_{n}^{\text {mode }}(t)=c_{1 n}^{2} \int_{-R}^{+R}(R-|r|) Z(r, t) d r
$$

Mode Travel Time Covariance

$$
v_{n}^{\text {mode }}(t)=d_{1 n}^{2} \int_{-R}^{+R}(R-|r|) Z(r, t) d r
$$

Ray Phase Covariance

$$
\vartheta_{n}^{\text {ray }}(t)=\left(2 k_{0} \sin \theta_{n}\right)^{2} \sum_{m=-M}^{+M}(M-|m|) Z\left(r_{m}, t\right)
$$

Ray Travel Time Covariance

$$
v_{n}^{\mathrm{ray}}(t)=\left(2 c_{0}^{-1} \sin \theta_{n}\right)^{2} \sum_{m=-M}^{+M}(M-|m|) Z\left(r_{m}, t\right)
$$

Our ultimate goal is to determine the spectra of the sea surface from the measured acoustic phase or travel time spectra. Before we are able to reach that goal, we review, in Chapter 3 , the statistical and spectral description of a two dimensional random surface. One then takes a Fourier transform of the covariances shown above to reach the goal of predicting surface wave frequency-directional spectra. 


\section{Chapter 3}

\section{Surface Wave Spectra - The Forward Problem}

In Chapter 2, we showed that we could determine the acoustic phase or travel time covariance given the sea surface covariance. In this chapter, the frequencydirection spectrum is shown to be related to the cross spectrum of the surface waves, i.e. the time Fourier transform of the sea surface covariance. Therefore, we can relate the spectra of our measurement to the sea surface spectra.

Our measurement is assumed to be the travel time or phase of an acoustic arrival, either in a full wave formalism, i.e. modes, or in the geometrical optics approximation, i.e. rays. The continuous sampling of the surface by the acoustic mode and the discrete sampling of the surface by a ray have consequences in how we solve for the sea surface frequency-direction spectrum. In the mode case, we make an analogy to a continous antenna while for the rays, we make an analogy to a discrete array of wave sensors. In either case, we will show we can solve for

the homogeneous (range-independent) and stationary (time-independent) spectrum directly from the measured acoustic spectra.

In 1984, MIZEX ' 84 (the Marginal Ice Zone Experiment, 1984) was performed in the Greenland Sea off Spitzbergen. One part of that experiment was the transmission of acoustic signals from a tomographic source and measurement of these signals at two different locations.[56] We apply the theory described here to signals 
measured at one of those locations.

In this chapter, Section 3.1 reviews the spectral description of the sea surface. Section 3.2 discusses the frequency-direction spectrum. Section 3.3 deals with mode phase and travel time spectra and their relation to the frequency-direction spectrum. Section 3.4 addresses the ray phase and travel time spectra. Section 3.5 describes the results of MIZEX '84 that deal with surface wave tomography. Finally, Section 3.6 quantifies the error incurred in the tomographic estimate of the frequency-direction spectrum under the frozen approximation assumption.

\subsection{Surface Wave Spectra Review}

In this section, we review the the statistical and spectral description of sea surface waves following Phillips [18]. We will denote the random sea surface displacement away from the mean height by $\xi\left(\vec{x}, t_{0}\right)$, a function of horizontal displacement $\vec{x}=(x, y)$ and time $t_{0}$.

The covariance of the zero-mean sea surface displacement field is

$$
\begin{aligned}
z\left(\vec{x}, \vec{r} ; t_{0}, t\right) & =\int_{-\infty}^{+\infty} \int_{-\infty}^{+\infty} \xi_{1} \xi_{2} P\left(\xi_{1}, \xi_{2}\right) d \xi_{1} d \xi_{2} \\
& =\left\langle\xi\left(\vec{x}, t_{0}\right) \xi\left(\vec{x}+\vec{r}, t_{0}+t\right)\right\rangle
\end{aligned}
$$

where the points 1,2 are taken as $\left(\vec{x}, t_{0}\right),\left(\vec{x}+\vec{r}, t_{0}+t\right)$ respectively and $P\left(\xi_{1}, \xi_{2}\right)$ is the joint probability distribution. This probability distribution

$$
P\left(\xi_{1}, \xi_{2}\right) d \xi_{1} d \xi_{2}
$$

represents the probability that the surface displacements at the points $\left(\vec{x}_{1}, t_{1}\right)$ and $\left(\vec{x}_{2}, t_{2}\right)$ should jointly lie within assigned limits $\left(\xi_{1}, \xi_{1}+d \xi_{1}\right)$ and $\left(\xi_{2}, \xi_{2}+d \xi_{2}\right)$. The mean square surface displacement is

$$
z\left(\vec{x}, 0 ; t_{0}, 0\right)=\int_{-\infty}^{+\infty} \xi_{1}^{2} P\left(\xi_{1}\right) d \xi_{1}=\sigma_{\xi}^{2} .
$$


If the wave field is homogeneous in space and stationary in time, the covariance is independent of $\left(\vec{x}, t_{0}\right)$.

The wave spectrum is the Fourier transform in the two spatial coordinates and in time of the sea surface covariance $Z(\vec{r}, t)$, i.e.

$$
X(\vec{K}, \Omega)=\frac{1}{(2 \pi)^{3}} \iiint Z(\vec{r}, t) e^{-i(\vec{K} \cdot \vec{r}-n t)} d \vec{r} d t
$$

where the integration is over the horizontal $\vec{r}$-plane and over all time $t$. The conjugate transform variables are spatial wavenumber vector $\vec{K}$ and temporal frequency $\Omega$, respectively. Frequency and wavenumber are related by a dispersion relation appropriate to infinitesimal linear gravity waves as (see [57] for a derivation)

$$
\Omega^{2}=g K \tanh K H
$$

where $H$ is the depth of the ocean. If $K H \rightarrow \infty$, Eq. (3.139) reduces to

$$
\Omega^{2}=g K,
$$

the "deep water" approximation. When $K H \rightarrow 0$, Eq. (3.139) becomes

$$
\Omega=\sqrt{g H} K
$$

which is the classic, nondispersive, long-wave approximation.

The inverse relation for Eq. (3.138) is

$$
Z(\vec{r}, t)=\iiint X(\vec{K}, \Omega) e^{+i(\vec{K} \cdot \vec{r}-\Omega t)} d \vec{K} d \Omega
$$

In particular, the mean square surface displacement is also given by

$$
\sigma_{\xi}^{2}=\iiint X(\vec{k}, \Omega) d \vec{k} d \Omega
$$

Of much interest to this thesis is the cross-spectrum, which is the Fourier transform in time of the covariance between sea surface displacements at points separated in space by $\vec{r}$

$$
\hat{\Phi}(\vec{r}, \Omega)=\frac{1}{2 \pi} \int_{-\infty}^{+\infty} Z(\vec{r}, t) e^{i \Omega t} d t .
$$


The two-sided frequency spectrum is a special case of the cross spectrum when $\vec{r}=0$, i.e.

$$
\hat{\Phi}(\Omega)=\frac{1}{2 \pi} \int_{-\infty}^{+\infty} Z(0, t) e^{i \Omega t} d t .
$$

Since the frequency spectrum in Equation (3.145) is even, we can define the traditional one-sided frequency spectrum as.

$$
\Phi(\Omega)= \begin{cases}2 \hat{\Phi}(\Omega) & \text { if } \Omega \geq 0 \\ 0 & \text { otherwise }\end{cases}
$$

The frequency spectrum, defined in Eq. (3.146), has been the object of study by many researchers because it is simple to measure: a point measurement in space and a spectrum of the time series output of a pressure sensor, wave staff, or other instruments fulfill this purpose.

\subsection{The Frequency-Direction Spectrum}

At this point, it is appropriate to review the frequency-direction spectrum $F(\Omega, \alpha)$ which describes the directional and frequency distribution of wave energy. Section 3.2.1 reviews the frequency-direction spectrum and its general properties. In Section 3.2.2, a model is reviewed which has been proposed by Donelan et al.[27] for the frequency-direction spectrum. This model is used later in this thesis to generate synthetic frequency-direction spectra. This spectrum is used to produce synthetic acoustic signal fluctuation spectra. The acoustic signal fluctuation spectra are then tomographically "inverted" to reproduce the orignal underlying surface wave spectra. 


\subsubsection{Spectrum Features}

The frequency-direction spectrum $F(\Omega, \alpha)$ is defined as

$$
F(\Omega, \alpha)=2 \int_{0}^{\infty} X(\vec{K}, \Omega) K d K
$$

where $\vec{K}=(K \cos \alpha, K \sin \alpha), \Omega$ is radial frequency, and $\alpha$ is direction. When we assume the linear deep water dispersion relation from Eq. (3.140), the frequencydirection spectrum is approximately

$$
F(\Omega, \alpha) \propto 2 \int_{0}^{\infty} \delta\left(K-\Omega^{2} / g\right) X(\vec{K}, \Omega) K d K
$$

The frequency-direction spectrum, as the name implies, is a two-dimensional "polar" spectrum. It can be interpreted as the distributions of $\sigma_{\xi}^{2}$ among waves with different frequencies $\Omega$ and directions $\alpha$ of propagation. The variance of the sea surface is therefore related to the frequency-direction spectrum as

$$
\sigma_{\xi}^{2}=\int_{0}^{2 \pi} \int_{0}^{\infty} F(\Omega, \alpha) d \Omega d \alpha
$$

If we assume the deep-water dispersion relation from Eq. (3.140), we can write the cross spectrum in terms of the frequency-direction spectrum as

$$
\hat{\Phi}(\vec{r}, \Omega)=\int_{0}^{2 \pi} F(\Omega, \alpha) e^{i \Omega^{2}(r / g) \sin \alpha} d \alpha
$$

where $\alpha$ is the angle measured from the perpendicular to $\vec{r}$ so that

$$
\vec{K} \cdot \vec{r}_{0}=K r \sin \alpha=\frac{\Omega^{2} r}{g} \sin \alpha .
$$

We can also write the one-sided frequency spectrum $\Phi(\Omega)$ as a special case of Eqs. (3.150) and (3.146) with $\vec{r}=0$, i.e.

$$
\Phi(\Omega)=\int_{0}^{2 \pi} F(\Omega, \alpha) d \alpha
$$




\subsubsection{A Model Spectrum}

In a paper by Donelan, Hamilton, and Hui[27], a useful frequency-direction spectrum model for wind-generated surface waves was deduced from data obtained using a wave staff array in Lake Ontario. The frequency spectrum portion of the model was essentially a correction of the JONSWAP model[24], while a completely new directional dependence was proposed based on the $\operatorname{sech}^{2}$ function. The model is given as

$$
F(\Omega, \alpha)=\frac{1}{2} \Phi(\Omega) \beta \operatorname{sech}^{2}\{\beta[\alpha-\bar{\alpha}(\Omega)]\}
$$

where $\bar{\alpha}$ is the mean wave direction and

$$
\beta=\left\{\begin{array}{lr}
2.61\left(\frac{\Omega}{\Omega_{p}}\right)^{+1.3} & 0.56<\frac{\Omega}{\Omega_{p}}<0.95 \\
2.28\left(\frac{\Omega}{\Omega_{p}}\right)^{-1.3} & 0.95<\frac{\Omega}{\Omega_{p}}<1.6 \\
1.24 & \text { otherwise }
\end{array}\right.
$$

The frequency spectrum is given by

$$
\Phi(\Omega)=\eta g^{2} \Omega^{-5} \frac{\Omega}{\Omega_{p}} e^{-\left(\frac{\Omega_{p}}{\Omega}\right)^{4}} g^{\Gamma}
$$

where $\Omega_{p}$ is the frequency of the spectral peak. The equilibrium range or rear face parameter $\eta$ is given by

$$
\eta=0.006\left(\frac{U_{s}}{c_{p}}\right)^{0.55} \quad 0.83<\frac{U_{c}}{c_{p}}<5
$$

The peak enhancement factor $\varrho$ is given by

$$
\varrho=\left\{\begin{array}{lr}
1.7 & 0.83<\frac{U_{c}}{c_{p}}<1 \\
1.7+6 \log \frac{U_{c}}{c_{p}} & 1<\frac{U_{c}}{c_{p}}<5
\end{array}\right.
$$

while the peak enhancement exponent $\Gamma$ is given by

$$
\Gamma=e^{-\frac{\left(\Omega-\Omega_{p}\right)^{2}}{2 \sigma^{2} \Omega_{p}^{2}}}
$$


The peak width parameter $\sigma$ is

$$
\sigma=0.08\left[1+4\left(\frac{c_{p}}{U_{c}}\right)^{3}\right]
$$

where $U_{c}$ is the component of the average wind velocity $10 \mathrm{~m}$ above the mean surface level in the mean direction of the waves at the peak of $\Phi(\Omega)$, and $c_{p}$ is the phase velocity of the waves at the peak of $\Phi(\Omega)$.

The relationship between phase velocity $c_{p}$ and fetch is given by [27] as

$$
\frac{U_{c}}{c_{p}}=11.6 \tilde{x}^{-0.23}
$$

where the non-dimensional fetch is $\tilde{x}=x g / U_{c}^{2}$. Using the deep water dispersion relation, we can solve for the peak frequency as a function of fetch $x$ as

$$
\Omega_{p}=11.6 \frac{g}{U_{\mathrm{c}}} \tilde{x}^{-0.23}
$$

Although the above model seems complicated, it is easily generated on the computer. Figure 3.1 shows a typical frequency-direction spectrum in a contour plot format for a wind speed of $10 \mathrm{~m} / \mathrm{s}$ and a fetch of $90 \mathrm{~km}$. A realization of this spectrum is shown in Fig. 3.2. Note that the waves are generally heading to the right with the wind and that the correlation distance is much greater left-to-right than top-to-bottom.

\subsection{Mode Phase and Travel Time Spectra}

We derived the equations relating the covariances of mode phase and travel time to the sea surface covariance in Section 2.1.3. In Section 3.1, we reviewed the properties of the sea surface covariance. In particular, we discussed the "crossspectrum": the time Fourier transform of the sea surface space-time covariance. The time Fourier transform of the mode phase and travel time covariances is simply the 


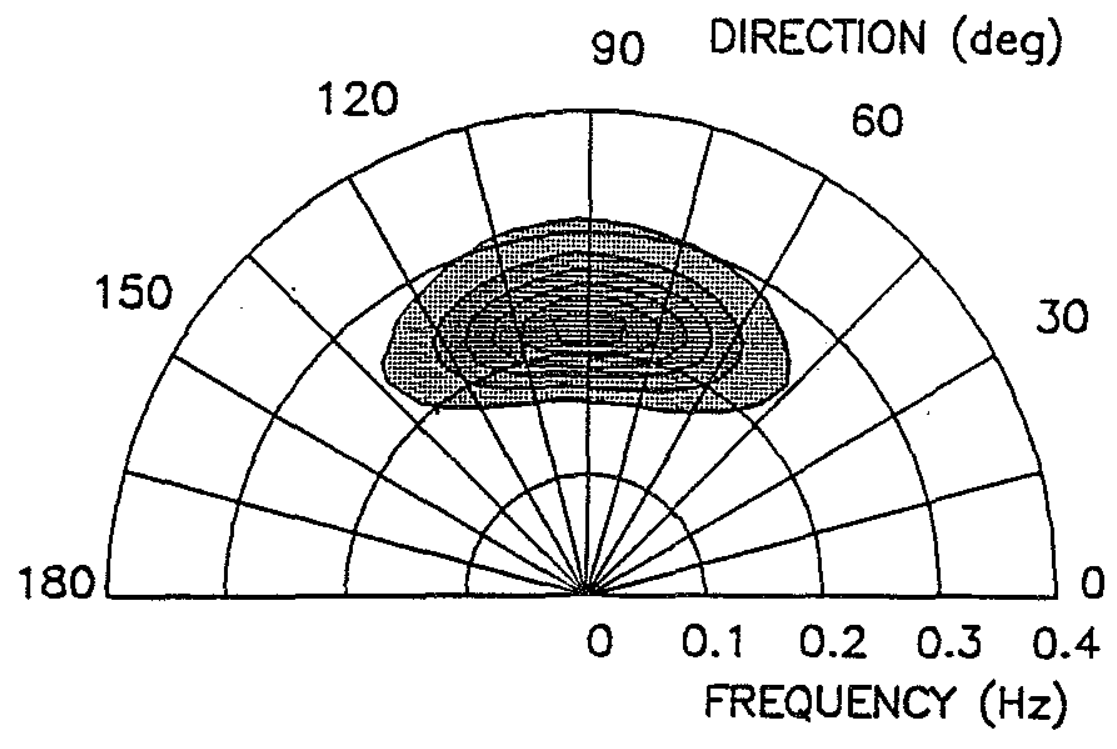

Figure 3.1: Typical frequency-direction spectrum. See Fig. 4.7 for grey scale.

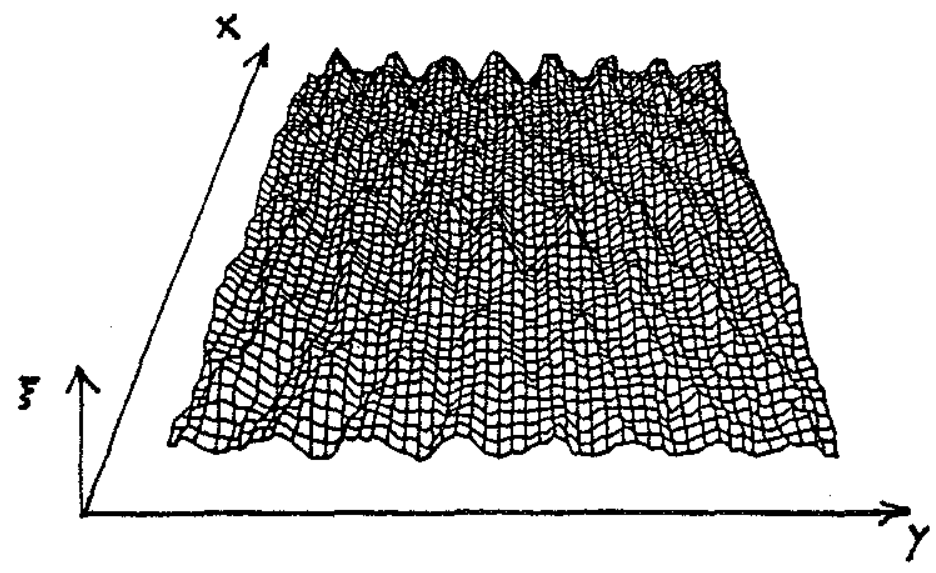

Figure 3.2: A realization of a sea surface from a typical frequency-direction spectrum. 
power spectra of the measurements[58]. This section carries out the time Fourier transform of the covariances, and using the properties of the cross-spectrum from Section 3.1, derives equations relating measured phase and travel time power spectra and sea surface frequency-direction spectra. Throughout this section we assume a stationary and homogeneous frequency-direction spectrum for the sea surface. Chapter 4 solves for the spatially dependent spectral problem.

Phase The time Fourier transform of the $n^{\text {th }}$ mode phase perturbation covariance from Equation (2.36) is

$$
\Theta_{n}^{\text {mode }}(\Omega)=c_{1 n}^{2} \int_{-R}^{+R}(R-|r|) \hat{\Phi}(r, \Omega) d r
$$

where now $\Theta_{n}^{\text {mode }}(\Omega)$ is the power spectrum of the nth mode phase perturbation and $\hat{\Phi}(r, \Omega)$ is the cross-spectrum of the sea surface as defined in Equation (3.144). Here we assume again that the surface waves obey the deep water dispersion relation $\Omega^{2}=g K$ so that using Equation (3.150) in Equation (3.162) we get

$$
\Theta_{n}^{\text {mode }}(\Omega)=c_{1 n}^{2} R^{2} \int_{0}^{2 \pi} \operatorname{sinc}^{2}\left[\frac{\Omega^{2} R \sin \alpha}{2 g}\right] F(\Omega, \alpha) d \alpha
$$

where $\operatorname{sinc}^{2}(x) \equiv \frac{\sin ^{2} x}{x^{2}}$. The mode phase spectrum $\Theta_{n}^{\text {mode }}(\Omega)$ can be interpreted as the output of an antenna with a triangular taper (or a Bartlett window [59]). The signal in this case is the frequency-direction spectrum $F(\Omega, \alpha)$. The main lobe of the antenna pattern is sensitive to waves coming from the direction perpendicular to the mode path, i.e. at the zeroes of the operand of the $\operatorname{sinc}^{2}$. Note that waves coming from $\alpha=0$ and $\alpha=\pi$ both give that same response. This left-right ambiguity is a common feature of line antennae.

We can write the frequency-direction spectrum as

$$
F(\Omega, \alpha)=\Phi(\Omega) h(\alpha ; \Omega)
$$




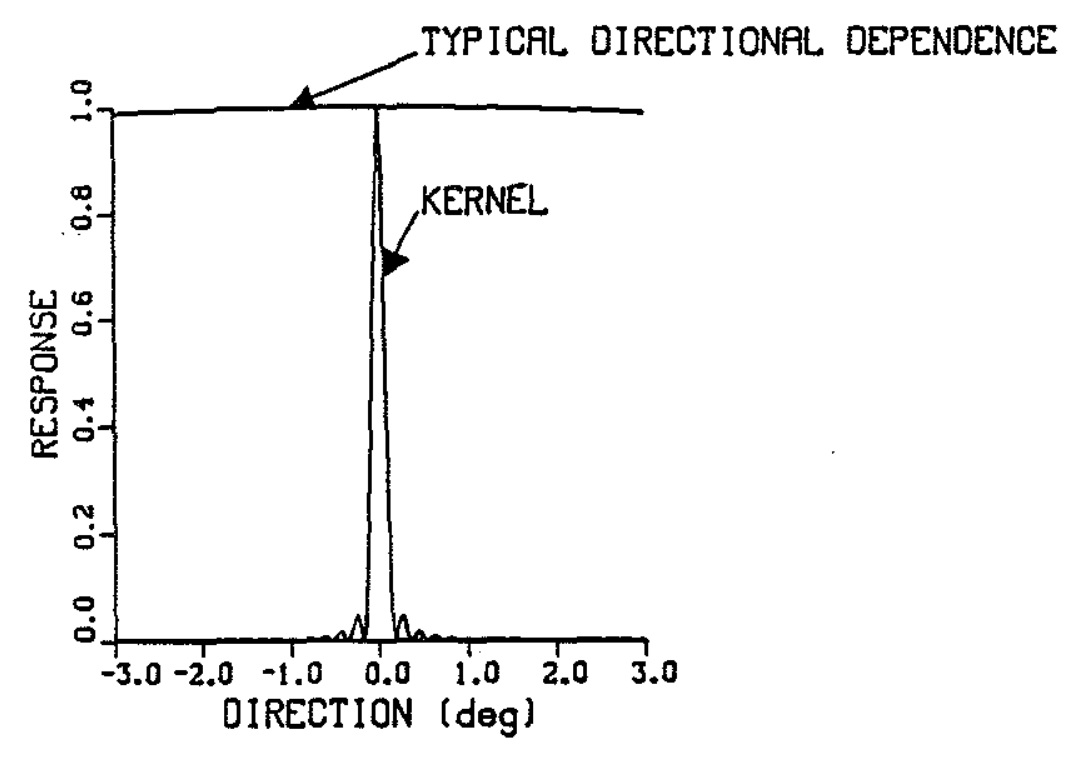

Figure 3.3: Typical variation in directional dependence and kernel; $R=50 \mathrm{~km}$, $\Omega=.1 \mathrm{~Hz}, \alpha_{c}=0$, and $\beta=2$.

where $\Phi(\Omega)$ is defined in Eq. (3.146) and $h(\alpha ; \Omega)$ is the directional dependence of the spectrum with the property that $\int_{0}^{2 \pi} h(\alpha ; \Omega) d \alpha=1$ for all $\Omega$. A reasonable but simple model for $h(\alpha ; \Omega)$ for purely wind-driven seas (i.e. no swell from distant storms) has been found to be [27]

$$
h(\alpha ; \Omega)=\frac{\beta}{2} \operatorname{sech}^{2}\left[\beta\left(\alpha-\alpha_{c}\right)\right]
$$

where $\alpha_{c}$ is the primary direction of the waves relative to the perpendicular to the mode path. $\beta$ is a weak function of frequency $\Omega$, approximately equal to 2 , and given exactly by Eq. (3.154).

Equation (3.163) can be simplified by noting the characteristics of the $\operatorname{sinc}^{2}$ kernel and the typical directional dependence in Eq. (3.165). The sinc ${ }^{2}$ term is very small away from $\alpha=0$. The $\operatorname{sech}^{2}$ term varying on a much slower scale around $\alpha=0$. Figure 3.3 shows the variation in the two terms. The variation in the directional dependence of the spectra is barely noticeable while the kernel's variation is confined to a very small region around $\alpha=0$. The consequence of this 
is that the frequency-direction spectra can be assumed constant within the integral in Eq. (3.163) so we can write

$$
\Theta_{n}^{\text {mode }}(\Omega) \simeq c_{1 n}^{2} R^{2} F(\Omega, 0) \int_{0}^{2 \pi} \operatorname{sinc}^{2}\left[\frac{\Omega^{2} R \sin \alpha}{2 g}\right] d \alpha .
$$

At this point, we note that $\frac{\Omega^{2} R}{g}=K R \gg 1$ for all reasonable wave frequencies and acoustic transmission ranges so that we can approximate $\sin \alpha$ by $\alpha$. Equation (3.166) now becomes

$$
\Theta_{n}^{\text {mode }}(\Omega) \simeq c_{1 n}^{2} R^{2} F(\Omega, 0)\left(\frac{2 g}{\Omega^{2} R}\right)^{2} \int_{-\infty}^{\infty} \alpha^{-2} \sin ^{2}\left(\frac{\Omega^{2} R \alpha}{2 g}\right) d \alpha .
$$

The definite integral in Eq. (3.167) can be evaluated analytically as[60]

$$
\int_{-\infty}^{\infty} \alpha^{-2} \sin ^{2}\left(\frac{\Omega^{2} R \alpha}{2 g}\right) d \alpha=\frac{\Omega^{2} R \pi}{2 g}
$$

Therefore, Eq. (3.167) becomes

$$
\Theta_{n}^{\text {mode }}(\Omega) \simeq \frac{2 \pi c_{1 n}^{2} R g}{\Omega^{2}} F(\Omega, 0) .
$$

We now can solve directly for the frequency-direction spectrum at a direction perpendicular to the source/receiver heading. The left/right ambiguity is still present in the problem but is suppressed for the moment.

Travel Time Taking the Fourier transform of the $n^{\text {th }}$ mode travel time perturbation covariance from Equation (2.45) we get

$$
\Upsilon_{n}^{\text {mode }}(\Omega)=d_{1 n}^{2} \int_{-R}^{+R}(R-|r|) \hat{\Phi}(r, \Omega) d r .
$$

where $\Upsilon_{n}^{\text {mode }}$ is the travel time fluctuation spectrum of mode $n$. We can write the relationship between travel time spectra and surface wave spectra exactly like we did for the phase, i.e.

$$
\Upsilon_{n}^{\text {mode }}(\Omega) \simeq \frac{2 \pi d_{1 n}^{2} R g}{\Omega^{2}} F(\Omega, 0)
$$




\subsection{Ray Perturbation Spectra}

Upward refracted rays sample the sea surface at discrete locations along the path from source to receiver (as contrasted to the continuously sampling modes). Instead of using a continuous antenna as an analogy, we use a discrete array analogy for the ray phase and travel time perturbation.

Phase Taking the Fourier transform of Equation (2.129), we get

$$
\Theta_{n}^{\text {ray }}(\Omega)=\left(2 k_{0} \sin \theta_{n}\right)^{2} \sum_{m=-M}^{M}(M-|m|) \hat{\Phi}\left(r_{m}, \Omega\right) .
$$

With our usual assumption of the deep water dispersion relation $\Omega^{2}=g K$ and using Equation (3.150), Equation (3.172) can be written

$$
\Theta_{n}^{\mathrm{ray}}(\Omega)=\left(2 k_{0} \sin \theta_{n}\right)^{2} \sum_{m=-M}^{M}(M-|m|) \int_{0}^{2 \pi} F(\Omega, \alpha) e^{i\left(\Omega^{2} / g\right) \Delta r m \sin \alpha} d \alpha .
$$

Using Equations (3.150) and (3.165) in Equation (3.173), and making the same assumptions used to derive the mode phase perturbation spectrum in Eq. (3.169), we get

$$
\Theta_{n}^{\text {ray }}(\Omega)=\frac{\left(2 k_{0} \sin \theta_{n}\right)^{2} M 2 \pi g}{\Omega^{2} \Delta r} \sum_{n} F\left(\Omega, \alpha_{n}\right)
$$

where $\alpha_{n}=\sin ^{-1}\left[\frac{2 \pi n g}{\Delta r \Omega^{2}}\right]$. Equation (3.174) can be interpreted as an output of an array of sensors, each located at every ray surface bounce location. The sum in the equation is due to the grating lobes that arise due to the periodicity of the array response. However the grating lobe locations in angle are dependent on frequency $\Omega$ and the exact position of the ray bounces. In the frequency averaging done in the spectrum estimation, only the central lobe is not averaged down, so that we can use Equation (3.174) to solve for the sea surface spectrum in terms of the ray phase perturbation spectrum

$$
\Theta_{n}^{\mathrm{ray}}(\Omega)=\frac{\left(2 k_{0} \sin \theta_{n}\right)^{2} M 2 \pi g}{\Omega^{2} \Delta r} F(\Omega, 0) .
$$


Also, ray bounce locations are seldom perfectly periodic in an actual experiment due to bathymetry and other effects, eliminating the concern over grating lobes.

Travel Time The travel time perturbations spectra of the rays can also be used to determine the frequency-direction spectra at the direction perpendicular to the source/receiver heading.

$$
\Upsilon_{n}^{\text {ray }}(\Omega)=\frac{\left(2 c_{0}^{-1} \sin \theta_{n}\right)^{2} M 2 \pi g}{\Omega^{2} \Delta r} F(\Omega, 0) .
$$

\subsection{MIZEX '84 Tomography Experiment}

During the 1984 summer Marginal Ice Zone Experiment (MIZEX '84), a $224 \mathrm{~Hz}$ acoustic tomography source was deployed off Spitzbergen, Norway, in $1200 \mathrm{~m}$ of water, sending out transmissions over the course of ten days (June 9-19,1984). The material in this section is primarily from a paper by Lynch, Spindel, Chiu, Miller, and Birdsall[56].

The experiment was designed to 1) investigate the general characteristics of acoustic propagation in the highly dynamic MIZ region, and 2) see if conditions were favorable for conducting large scale MIZ or Greenland Sea tomography experiments. During the data analysis, it was noticed that the phase spectrum (or equivalently the travel time fluctuation spectra of surface interacting rays) exhibited a shape similiar to that of the surface wave field. This suggested that surface wave tomography might be possible, a previously unexplored possibility, and one which only could have been seen in the rapidly sampled data set.

The source deployed in the experiment was a quarter-wavelength resonant tube with a center frequency of $224 \mathrm{~Hz}$ and a bandwidth of approximately $12 \mathrm{~Hz}$ (which gives a travel time resolution of $83 \mathrm{~ms}$ ). It was moored at coordinates $78^{\circ} 59.3 \mathrm{~N}$, $6^{\circ} 58.6 \mathrm{E}$ at $181 \mathrm{~m}$ depth in $1200 \mathrm{~m}$ of ocean. The mooring was tracked every 15 
min by three high frequency transponders moored near the bottm, so that any movement of the source could be accounted for when analyzing the data. the signal was a 63 digit phase encoded psuedorandom sequence which was repeated every 3.9375 seconds. This signal was designed to give large processing gain $(\sim 38 \mathrm{~dB})$ to an inherently low power system with bandwidth and peak power limitations, thus making long distance tomography feasible[4]. During the experimental period, the source was activated for a continuous two hour period, daily from 0000 GMT to 0200 GMT. In this thesis, we discuss two of the two hour transmissions, specifically those beginning at 0000 on year day 168 and 169 corresponding to June 16 and June 17, 1984.

The signals were recorded at two different locations, shown in Fig. 3.4. The nearest sensor, deployed by the Naval Underwater Systems Center (NUSC), located at $79^{\circ} 21.7 N, 8^{\circ} 31.42 E$, was $52.93 \mathrm{~km}$ distant along an ice-free path. (We are obliged to Dr. Fred DiNapoli for the data set.) The further sensor, deployed by MIT and Woods Hole (WHOI) and located at $80.405^{\circ} \mathrm{N}, 8.58^{\circ} \mathrm{E}$ (at $0000 \mathrm{Z}$, June 17), was suspended at $60 \mathrm{~m}$ depth from the $M / V$ Kvitbjorn. We will restrict our discussions to the signals received by the NUSC array.

Oceanographically, the MIZ region west of Spitzbergen, through which the tomography transmissions were sent, is dominated by the warm West Spitzbergen current flowing northward[61]. This surface current heats the upper few hundred meters of the water column, producing complicated sound speed profiles, such as those measured at our source and receiver positions (Figs. 3.5 and 3.6). Beneath about $600-800 \mathrm{~m}$, the effects of hydrostatic pressure dominate the sound speed, giving the usual deep ocean adiabatic gradient of $0.016 \mathrm{~s}^{-1}$.

The experiment was conducted on the Nansen Bank of the Yermak Plateau, where the bathymetry governing the propagation between the source and the two receivers was moderately shallow[62]. Figure 3.7 shows the bathymetry and the 


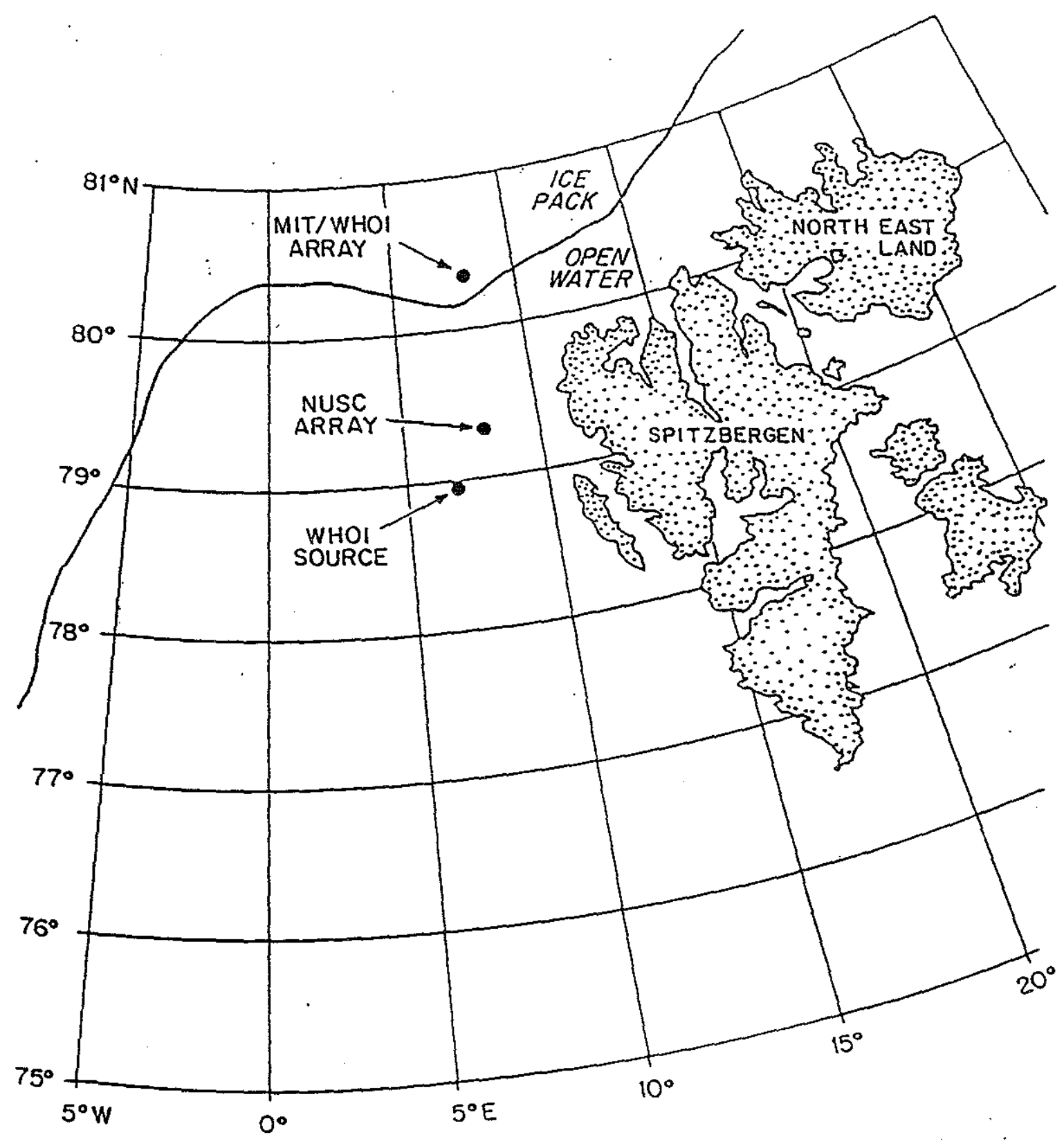

Figure 3.4: Geometry for the MIZEX '84 tomography experiment 


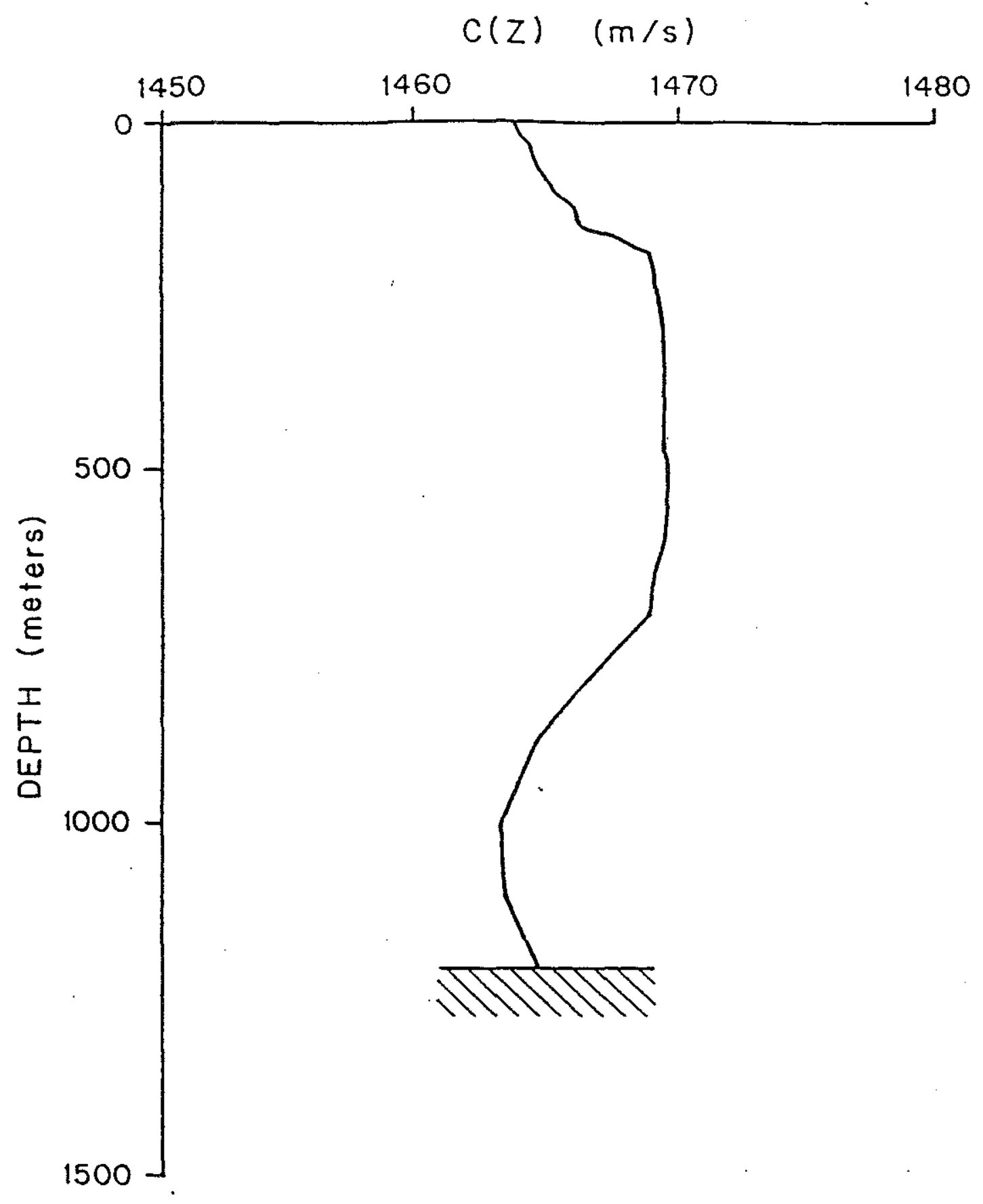

Figure 3.5: Sound velocity profile at the WHOI tomography source. 


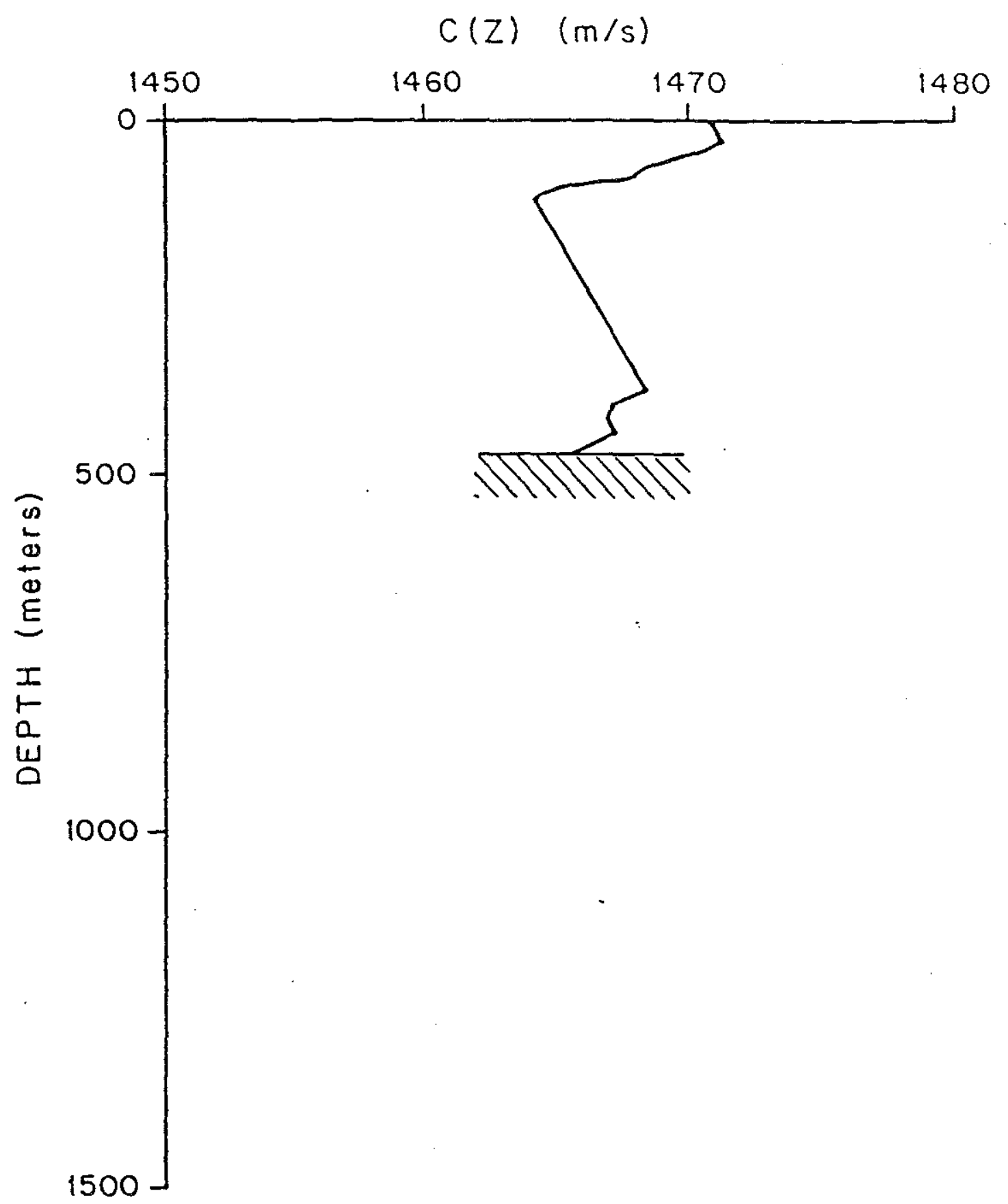

Figure 3.6: Sound velocity profile near the NUSC reciever. 


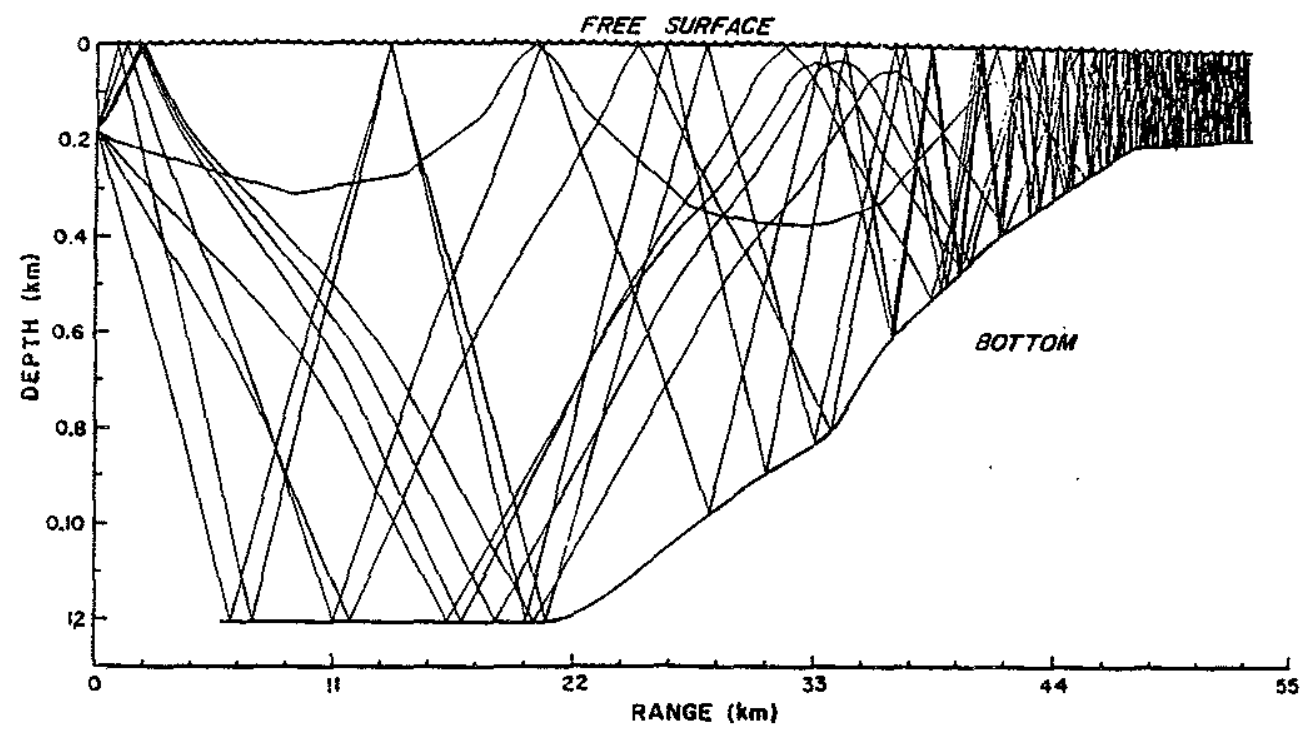

Figure 3.7: Range dependent ray trace between the WHOI source and the NUSC receiver.

results of a ray trace program using sound speed profiles that were interpolated between source and receiver. Between the source and the NUSC array, the slope is more pronounced, going from 1200 to $180 \mathrm{~m}$ over $36 \mathrm{~km}$. All rays received interacted with the bottom and the sea surface a number of times.

The interaction of the rays with the sea surface had an effect on their phase measured at the receiver. The phase for one of the rays is shown versus time from 0000 to 0200 GMT on day 168 in Fig. 3.8. The time series in that figure has two features worth noting. First, a slow sinusoidal-like variation with a period of about $90 \mathrm{~min}$ is evident. This slow variation is believed to be an internal wave signature. Second, on top of the slow variation, there is a very fast oscillation in the phase of the ray arrival. The fast variations are believed to be due to surface waves. Fig. 3.9 shows the power spectrum of the phase fluctuations where the two features have their spectral counterparts: internal wave induced energy at frequencies less than $.01 \mathrm{~Hz}$ and energy due to surface waves between $.08 \mathrm{~Hz}$ and the experimental 


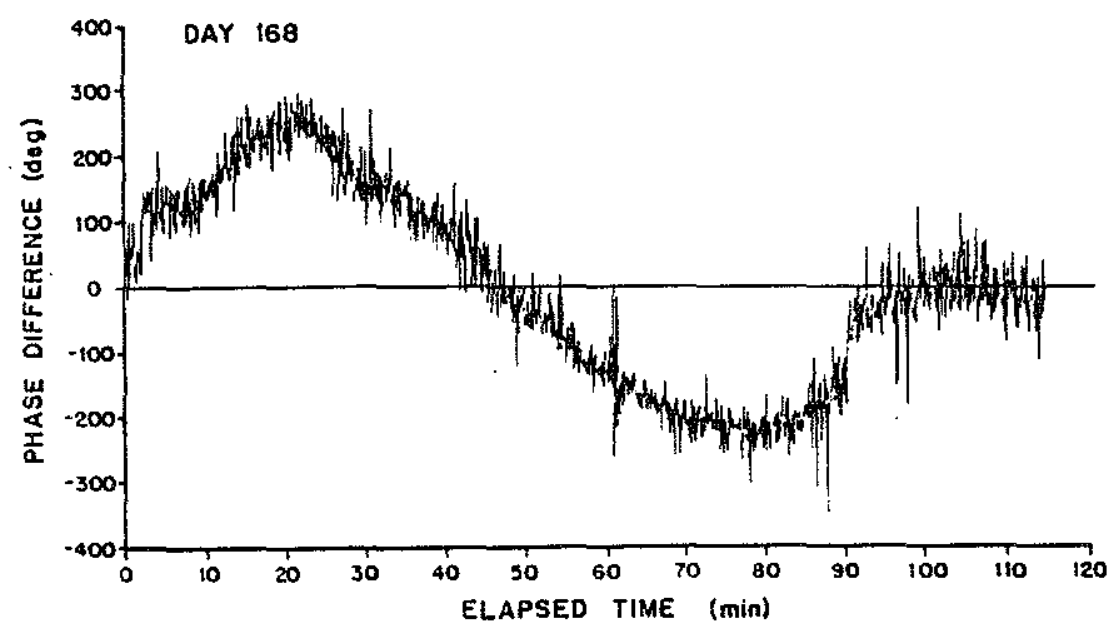

Figure 3.8: Phase of the prominent arrival at NUSC receiver on day 168 with mean subtracted out.

Nyquist frequency of about $.125 \mathrm{~Hz}$. Figures 3.10 and 3.11 show the same data for the next day. The energy located in the .08 to $.125 \mathrm{~Hz}$ band is greatly diminished on day 169 as compared to day 168 . To show this is due to surface waves, we estimate the sea state in the vicinity of the NUSC array from wind force measurements. On day 168, four foot (peak to peak) waves were estimated due to force 3 and 4 winds from the south and west (open fetch). On day 169, force 2 winds were from the north (about $100 \mathrm{~km}$ fetch) followed by force 1 winds from the south, giving less than one foot seas. The location (8-12 sec period) of energy in the phase fluctuation spectra along with the sea state measurements on the two days provides a convincing argument that the fluctuations are due to surface waves.

Applying Eq. (3.175) to the measured ray phase perturbation spectrum on two days (168 and 169) gives the results shown in Figure (3.12). Note that the day 169 spectrum is essentially flat, indicating a. calm day whereas the day 168 spectrum has appreciable energy. The latter spectrum also shows a double peak perhaps due 


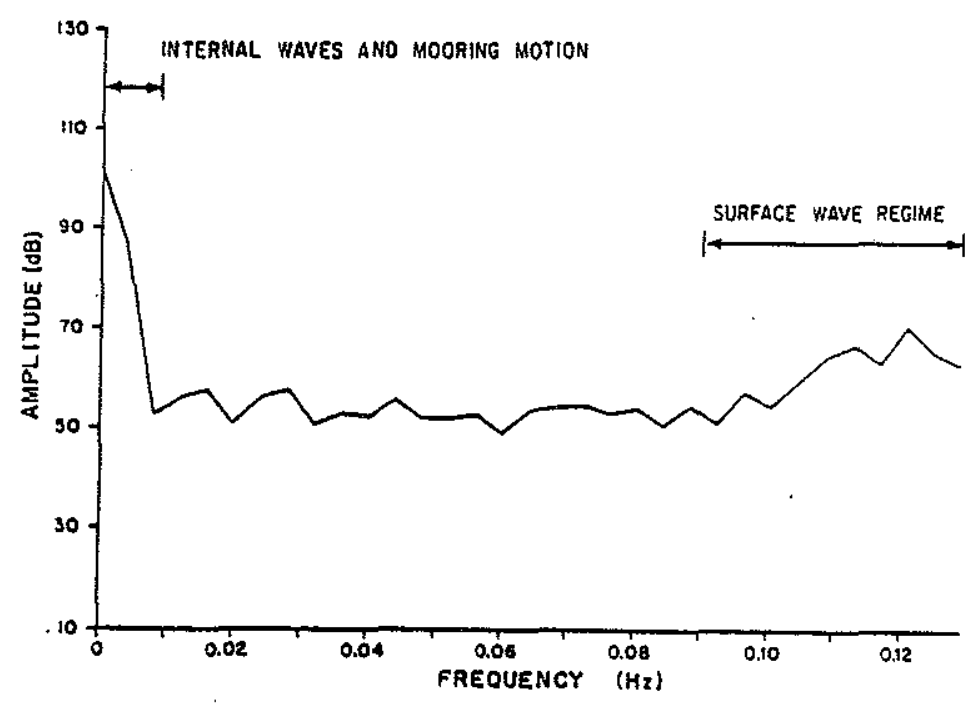

Figure 3.9: Spectrum of the phase of prominent arrival at NUSC receiver on day. 168.

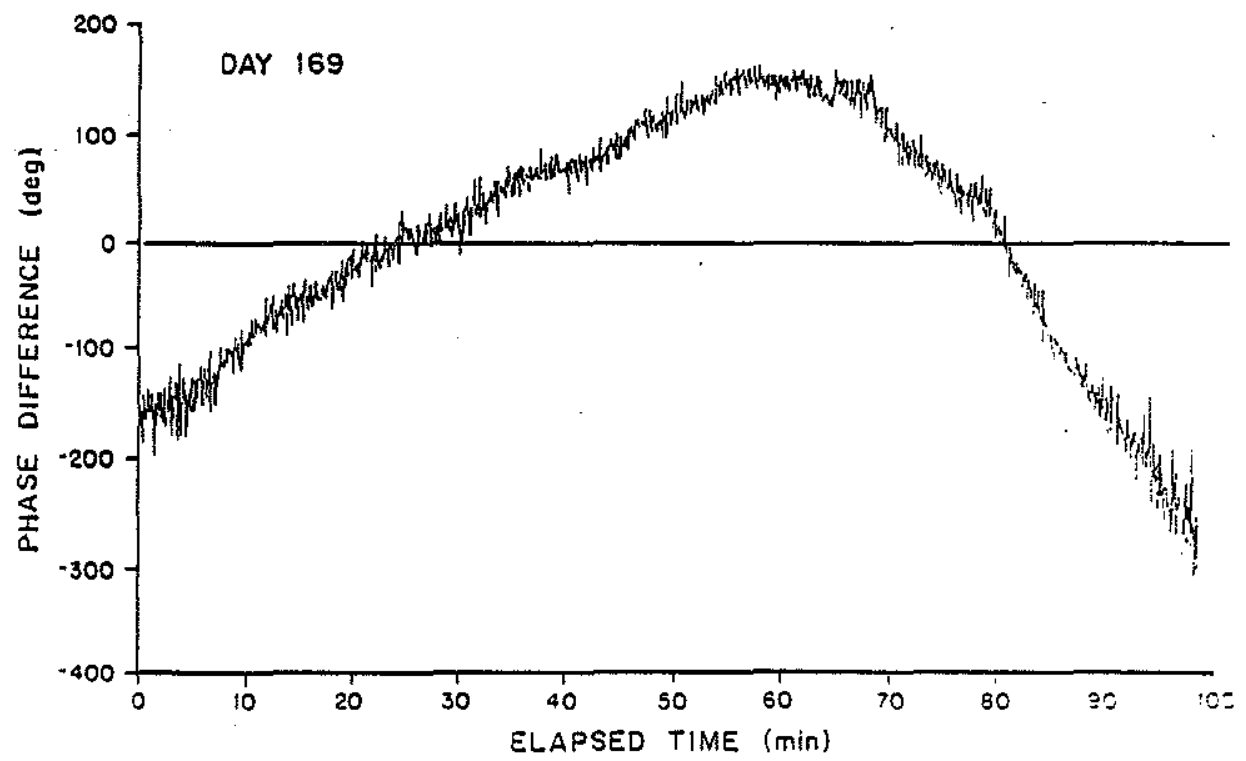

Figure 3.10: Phase of the prominent arrival at NUSC receiver on day 169 with mean subtracted out. 


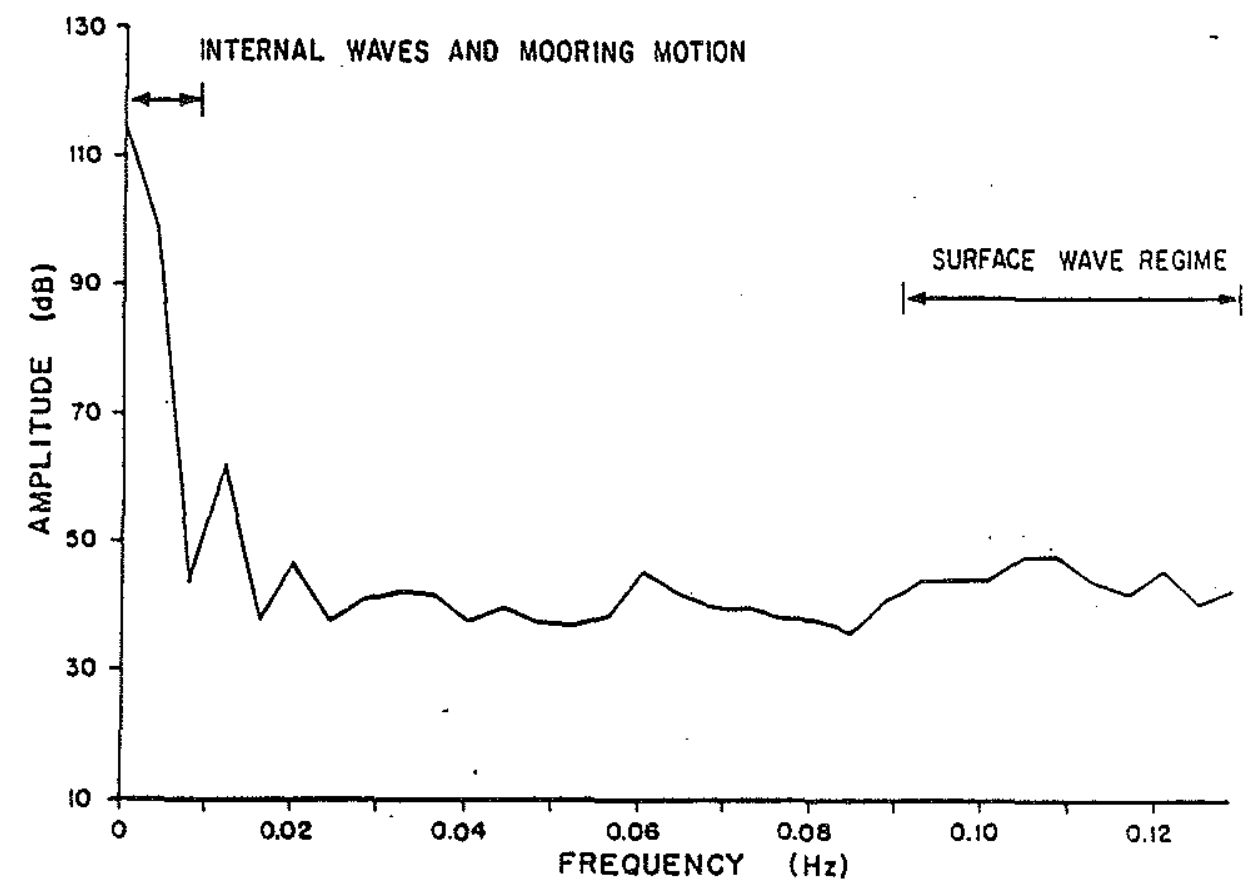

Figure 3.11: Spectrum of the phase of prominent arrival at NUSC receiver on day 169.

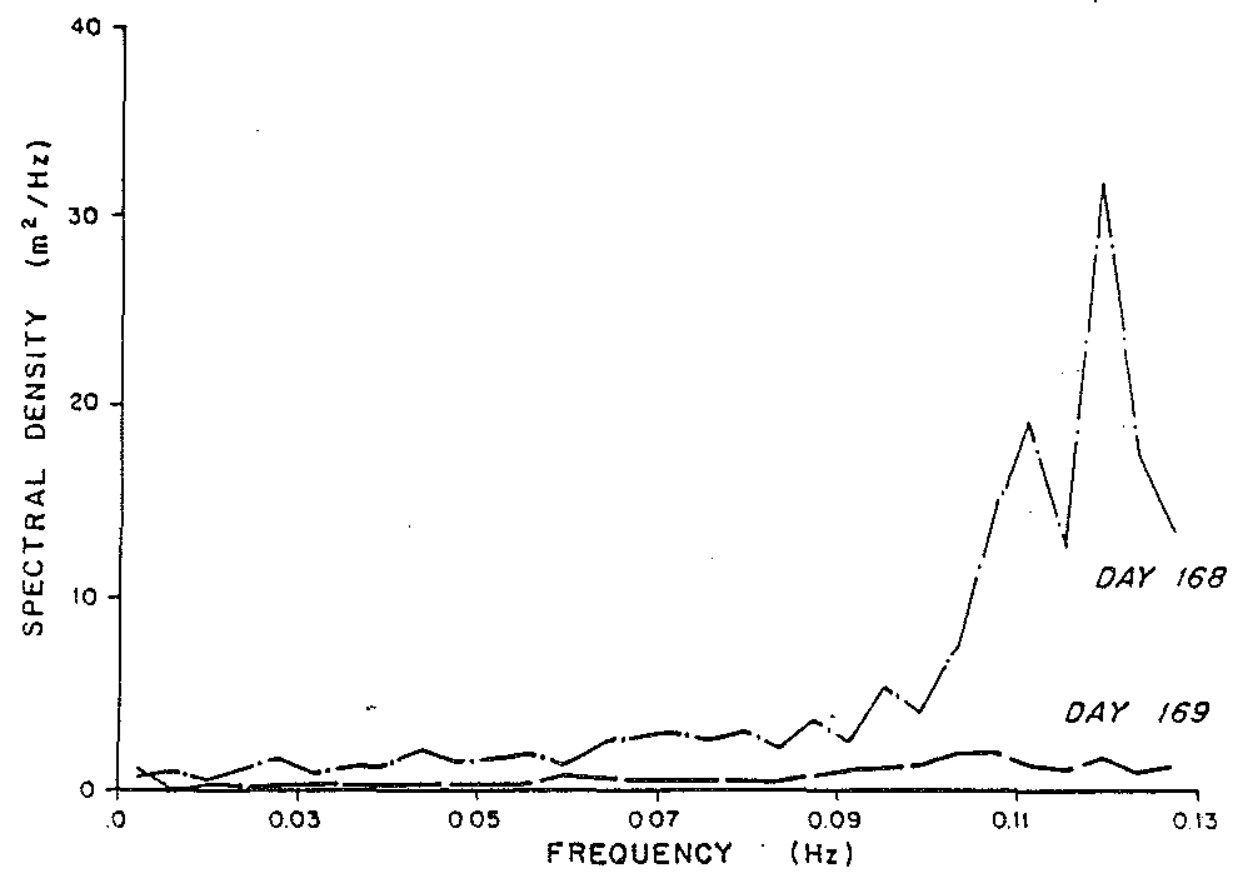

Figure 3.12: Surface wave spectra obtained for days 168 and 169 during MIZEX ' 84. 
to aliasing or to two low frequency wave trains coming from different directions. Since we only have one source/receiver pair, we do not have much confidence in the absolute vertical scale of Figure (3.12). However, the shape and location of the spectral energy resembles the general shape of sea surface wave spectra measured by other workers. $[27,63]$

\subsection{The Frozen Approximation}

The frozen approximation consists of assuming the sea surface is not moving for the time of flight of the acoustic ray or mode. Here, we quantify the error incurred in the tomographic estimate of the frequency-direction spectrum under the frozen approximation assumption.

As we showed earlier in this chapter, the acoustic phase and travel time perturbations are greatest for waves whose crests are parallel to our acoustic path for the frozen surface. Mode $n$ has a finite group velocity given by

$$
v_{g n}^{\text {mode }}=\frac{\partial \omega}{\partial \kappa_{n}}
$$

where $\omega$ is the acoustic frequency and $\kappa_{n}$ is the mode eigenvalue. The group velocity of ray $n$ can be represented by

$$
v_{g n}^{r a y}=c \sin \theta_{n}
$$

where $\theta_{n}$ is the angle of incidence. If the surface is moving, the direction of the one surface wave (out of many in a direction spectrum), that the acoustic mode is sensitive to, is that which satisfies

$$
\sin \alpha=\frac{v_{p}}{v_{g n}^{\text {mode }}}
$$

where $v_{p}$ is the phase velocity of the surface wave and again $\alpha$ is measured from the perpendicular to the source/receiver heading. The frozen approximation consists of 


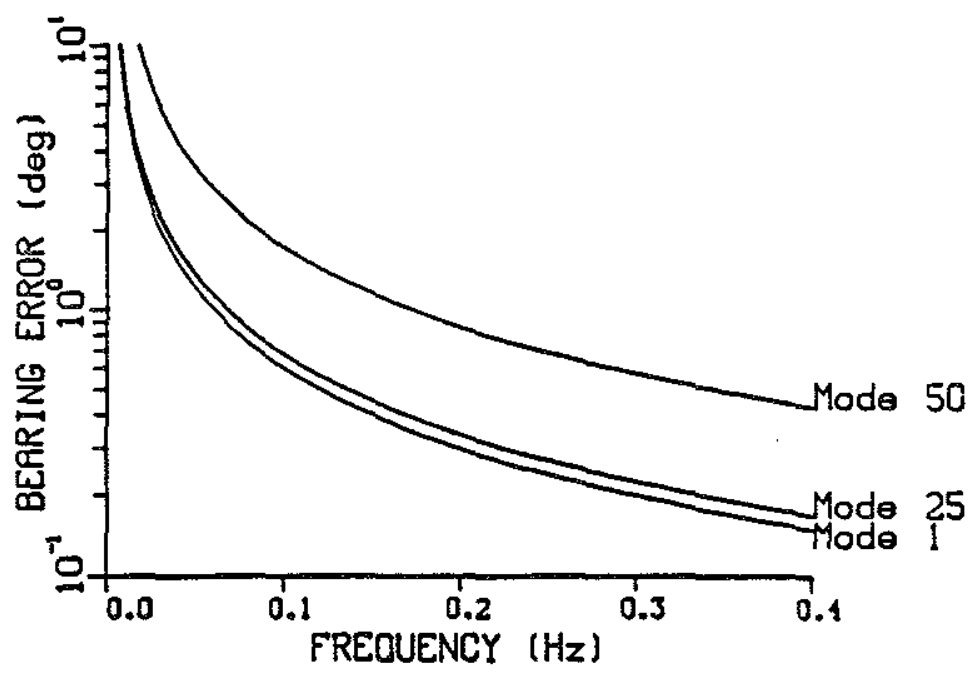

Figure 3.13: Frequency-dependent bearing error due to the frozen approximation. For a $180 \mathrm{~m}$ ideal waveguide, $220 \mathrm{~Hz}$ acoustic source frequency, and modes 1,25 , and 50 out of the total 53 propagating modes.

letting $v_{g n} \rightarrow \infty$, which "freezes" the surface for the time of flight of the mode or ray.

For a finite group velocity $v_{g n}$, the wave direction that perturbs the acoustic phase and travel time the most is that whose wave crests just meet the acoustic energy as it travels between source and receiver. That direction is slightly off the perpendicular, and for deep water waves with dispersion $\Omega^{2}=g K$, Eq. (3.179) is given by

$$
\sin \alpha=\frac{g}{\Omega v_{g n}^{\text {mode }}}
$$

Figure 3.13 shows this frequency-dependent bearing error for a $180 \mathrm{~m}$ ideal hard bottom waveguide, $220 \mathrm{~Hz}$ acoustic source frequency. Modes 1, 25, and 50 out of the total 53 propagating modes. Figure 3.13 tells us that for almost all propagating modes and wave frequencies above $.1 \mathrm{~Hz}$, the error is less than $1 \mathrm{deg}$. The frozen approximation is thus seen to be very adequate for our analyses. 


\section{Chapter 4}

\section{Wave Spectra Estimation with Tomography}

A major objective of this thesis is the estimation of sea surface wave spectra from acoustic tomography measurements. Chapter 2 described the effects of the rough sea surface on the tomographic signal. Chapter 3 showed how to derive the spectrum of the signal fluctuations from the frequency-direction spectrum of the sea surface, i.e. the forward problem. In this chapter, we address the inverse problem. Given the fluctuations in the signals transmitted from a number of acoustic sources to a number of receivers, we solve for the quasi-homogeneous, quasistationary frequency-direction spectrum of the sea surface waves that caused the fluctuations. By quasi-homogeneous, we mean that the characteristic spatial scale of the inhomogeniety is much greater than the longest wavelength of the surface waves. The quasi-stationary assumption means that the spectra may evolve in time on scales much longer than the longest wave period.

If the sea surface spectrum is homogeneous over the range of the transmission, we showed in Section 3.3 that the sea surface frequency-direction spectrum is an algebraic mapping of the mode phase or travel time pertubation spectrum measured; the source-receiver pair measures the components of the frequency-direction spectra in the direction perpendicular to the path in the $(x, y)$ plane. In this chapter, we solve the inverse problem for the spatially-varying frequency-direction spectrum 
given the tomographic signal spectra. The inversion can be repeated over time to retrieve time-evolving spectra.

Inversions are performed on both mode and ray travel time spectra, simulated in noise-free and noisy computer experiments. For a finite acoustic signal-to-noise ratio, error exists in the acoustic travel time estimates. The noise is assumed to be white and uncorrelated between transmissions. One of the features of the algebraic mapping from Section 3.3 was a multiplication of the measured phase spectrum by the $\Omega^{2}$. If the phase and travel time spectrum includes the white noise, this noise is amplified at high frequencies. The severity of this depends on the acoustic signal-to-noise ratio (SNR), and in the ray case, on the number of rays bounces as will be shown.

The organization of this chapter is as follows. Section 4.1 describes the inversions of mode travel time perturbation spectra. Section 4.2 deals with inversions of ray data, and Section 4.3 shows selected resolution and variance results.

\subsection{Inversion of Modes}

This section deals with a number of different topics. Section 4.1.1 introduces the mode inverse problem. Section 4.1.2 describes how we discretized the spatially dependent frequency-direction spectra. The solution technique we use in this thesis is described in Section 4.1.3. The tradeoff between resolution and variance to determine the unknown Lagrange parameters is illustrated in Section 4.1.5. Sections 4.1.2, 4.1.3, and 4.1.5 are applicable to both mode and ray inversions. Section 4.1.6 shows the inversion results using a single mode between each transceiver. 


\subsubsection{Posing the mode inverse problem}

Here, we assume the surface wave frequency-direction spectrum is spatially dependent. The algebraic relations (Eqs. (3.169) and (3.171)) between the phase and travel time spectra and the frequency-direction spectrum do not hold in this case. For a tomographic source located at $\vec{r}_{i}$ and a receiver at $\vec{r}_{j}$, Eq. (3.171) can be generalized to

$$
\Upsilon_{i j}^{\text {mode }}(\Omega)=\frac{d_{1}^{2} 2 \pi g}{\Omega^{2}} \int_{\vec{r}_{i}}^{\vec{r}_{i}} F\left(\Omega, \alpha_{i j}, \vec{r}\right) d \vec{r}+\hat{\varepsilon}
$$

where $\hat{\varepsilon}$ is an error term discussed below and we have left out the explicit dependence on mode number $n$ of $d_{1}$ and $\Upsilon_{i j}^{\text {mode }}$. We have assumed that the frequency-direction spectrum, $F\left(\Omega, \alpha_{i j}, \vec{r}\right)$, is a slow function of range.

A consequence of assuming quasi-homogeneous spectra is that spectral components are not independent as in the homogeneous case. (For a discussion of such nonstationary random processes, see the text by Bendat and Piersol [58].) The error term $\hat{\varepsilon}$ is proportional to the spectral component dependence. However, if we assume that the spatial scale of the spectrum is much greater than the longest wavelength in the spectrum, the dependence is weak and we may neglect $\hat{\varepsilon}$. Of course, as the spectrum approaches homogeniety at all ranges, the dependence disappears, i.e. $\hat{\varepsilon} \rightarrow 0$ and Eq. (4.181) becomes identical to Eq. (3.171). One could write a similiar relation to Eq. (4.181) based on Eq. (3.169) for the phase perturbation spectrum which would be the same except for the replacement of $d_{1}$ by $c_{1}$.

Note that all modes that turn at the sea surface have a similiar dependence on the frequency-direction spectrum. The only difference between spectra of one mode and another at this level of approximation is the coefficients $c_{1 n}$ for phase and $d_{1 n}$ for the travel time. This has important experimental ramifications for we could use any one of the propagating modes. The choice could be based on SNR, resolution, etc. However, for higher mode numbers, the approximation that the mode phase 


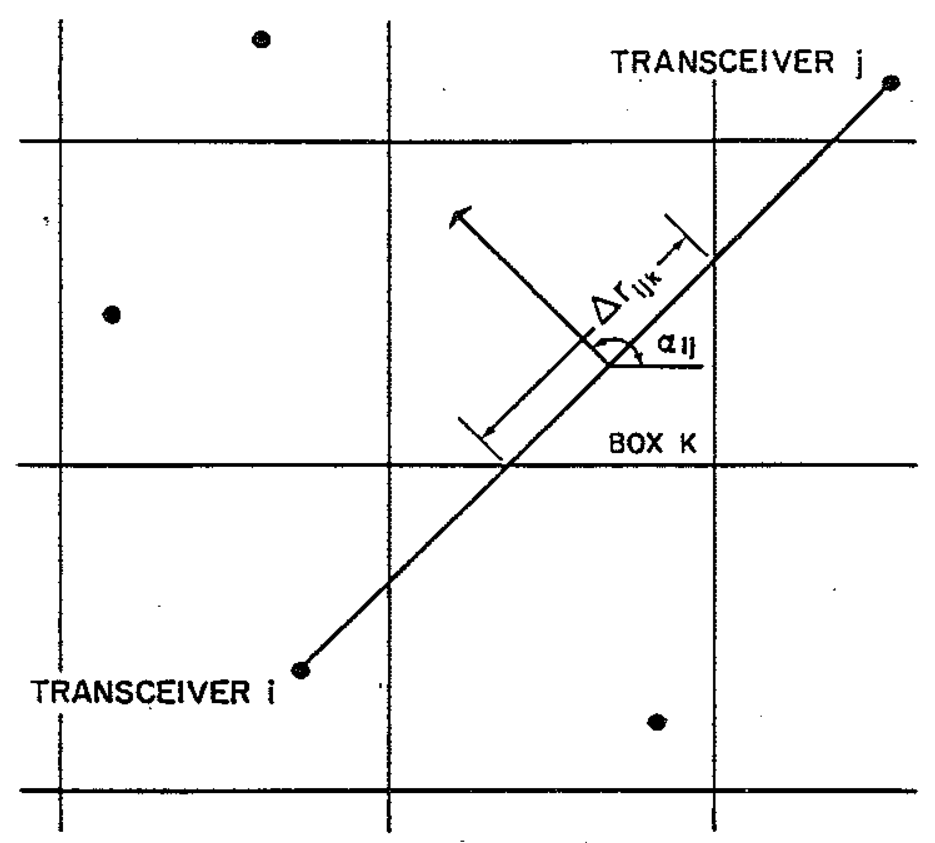

Figure 4.1: Geometry for discretizing the relation between travel time spectra and surface wave frequency-direction spectra. See Eq. (4.182).

and sea surface height are linearly related starts to breakdown, i.e. when $c_{1 n} \sim c_{2 n}$. Those modes would probably not be useful anyway because they would attenuated due to their high vertical wavenumber. There is no reason why we could not use multiple mode spectra in the inversion to improve noise tolerance. For simplicity, however, we restrict ourselves in this thesis to inversion of a single mode spectrum. measured at each receiver from each source.

The first step to solving Eq. (4.181) is to discretize the integral, i.e.

$$
\Upsilon_{i j}^{\text {mode }}(\Omega)=\frac{d_{1}^{2} 2 \pi g}{\Omega^{2}} \sum_{k} F\left(\Omega, \alpha_{i j} ; \vec{\tau}_{k}\right) \Delta r_{i j k}
$$

where $\Delta r_{i j k}$ is the distance the mode travels in the box whose center is at $\vec{r}_{k}$ at angle $\alpha_{i j}$. These quantities are illustrated in Fig. 4.1.

We then form a vector $f$ of the unknown frequency-direction spectra $F$ that is a function of box $k$ with center located $\vec{r}_{k}$ and sector $l$ with center located at angle 
$\hat{\alpha}_{l}$ as

$$
f(\Omega)=\left[\begin{array}{c}
F\left(\Omega, \hat{\alpha}_{1}, \vec{r}_{1}\right) \\
F\left(\Omega, \hat{\alpha}_{2}, \vec{r}_{1}\right) \\
\vdots \\
F\left(\Omega, \hat{\alpha}_{L}, \vec{r}_{1}\right) \\
F\left(\Omega, \hat{\alpha}_{1}, \vec{r}_{2}\right) \\
F\left(\Omega, \hat{\alpha}_{2}, \vec{r}_{2}\right) \\
\vdots \\
F\left(\Omega, \hat{\alpha}_{L-1}, \vec{r}_{K}\right) \\
F\left(\Omega, \hat{\alpha}_{L}, \vec{r}_{K}\right)
\end{array}\right]
$$

We can form a vector $t$ of the measured travel time fluctuation $\Upsilon_{i j}^{\text {mode }}$ measured at the $\mathrm{jth}$ receiver from the ith source.

$$
t(\Omega)=\left[\begin{array}{c}
\Upsilon_{11}^{\text {mode }}(\Omega) \\
\Upsilon_{12}^{\text {mode }}(\Omega) \\
\vdots \\
\Upsilon_{i j}^{\text {mode }}(\Omega) \\
\vdots \\
\Upsilon_{I J}^{\text {mode }}(\Omega)
\end{array}\right]
$$

The $t$ is a vector of length $M$. We assume that $\Upsilon_{i j}^{\text {mode }}=\Upsilon_{j i}^{\text {mode }}$, i.e. so that the vector $\mathrm{t}$ only includes the independent pairs. For example, if we have 8 transceivers, we have $7+6+5 \ldots+1=28$ different paths and therefore $M$ would be 28 .

We can now form the matrix equation

$$
\mathrm{t}=\frac{d_{1}^{2} 2 \pi g}{\Omega^{2}} \mathrm{Gf}+\mathbf{n}
$$

where $t$ is the vector of length $M$ made up of the travel time or phase perturbation spectra measured from each source to each receiver. 
The vector $\mathrm{f}$ is the unknown vector of length $N=L \times K$ of the frequencydirection spectrum at all discrete range boxes and angular sectors. Section 4.1.2 discusses the discretization of the frequency-direction spectrum. The matrix $G$ is the $M \times N$ kernel matrix that has all the geometrical information implicit in Eq. (4.182) and, importantly, is independent of frequency $\Omega$. The vector $n$ is a noise vector to be addressed below. For a realistic number of sources and receivers, and for a realistic range-dependent discrete model of the frequency-direction spectrum, the $N$ is greater than $M$, i.e. the problem is underdetermined.

\subsubsection{Model Discretization}

We have discretized the frequency-direction spectrum into vectors. The discretization used is 9 range boxes, each $20 \times 20 \mathrm{~km}$, and 18 angular sectors, each 10 deg, giving 162 unknown values of our frequency-direction spectra model to be determined at each frequencies $.01 \mathrm{~Hz}$ apart between $0 \mathrm{~Hz}$ and $.5 \mathrm{~Hz}$. Because of the left-right ambiguity described earlier, we will only invert for directions from 0 to 180 deg. Figure 4.2 illustrates the discretization used here. We have simulated a tomographic array of 8 transceivers on a $25 \mathrm{~km}$ radius circle within the 9 boxes. Figure 4.3 shows the locations of the 8 transceivers and all the acoustic mode paths between them. The data vector $t$ consists of the phase or travel time spectrum at each frequency measured at each of the transceiver locations shown in Fig. 4.3. The same generalized inverse is applied at each frequency.

Data was simulated using a frequency-direction spectra model described by Donelan et al.[27] using a modified JONSWAP[24] frequency spectrum and a sech ${ }^{2}$ directional dependence. The wind speed is taken to be $10 \mathrm{~m} / \mathrm{s}$ at a height of $10 \mathrm{~m}$ above the mean surface height. The wind direction is from $90 \mathrm{deg}$, i.e. off shore. The shore is $40 \mathrm{~km}$ from and parallel to the top boxes as shown in Fig. 4.4: 


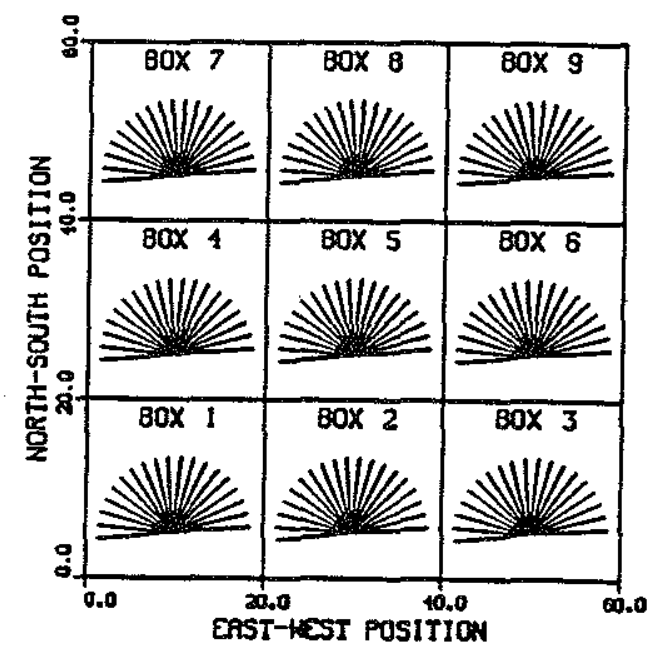

Figure 4.2: Frequency-direction spectrum angular and spatial discretization.

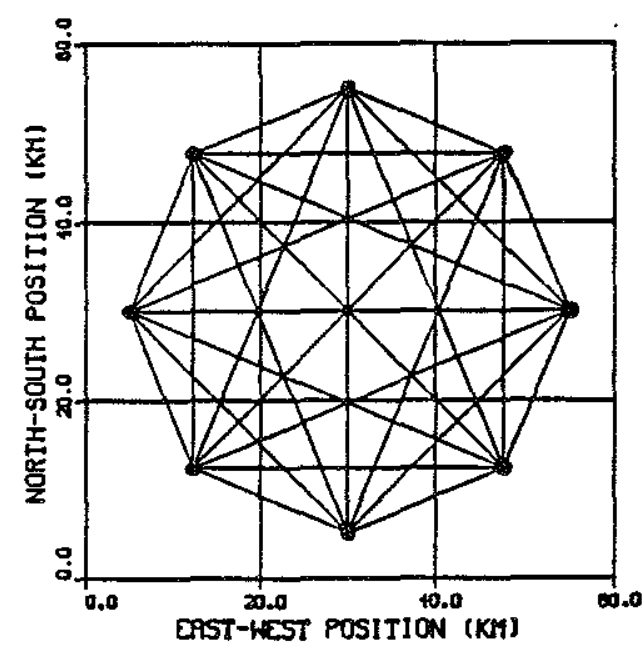

Figure 4.3: Tomographic array and acoustic mode paths. 


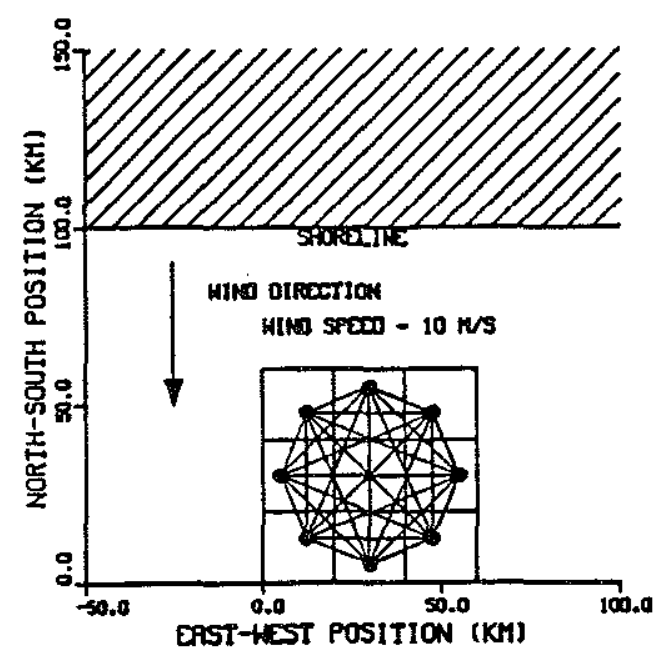

Figure 4.4: Tomographic array location.

The kernel matrix $\mathrm{G}$ can be interpreted in the following manner. The value of the $\mathrm{G}$ matrix entry is the distance traveled by the mode in each box received at the appropriate transciever. Figure 4.5 illustrates the $G$ matrix in a manner similiar to a beam pattern. The direction of each sector is perpendicular to the path of the mode. The radius of the sector is proportional to the distance the mode traveled in the box. The asymmetry of the pattern around $90 \mathrm{deg}$ is due to the fact that we discretized in direction with sectors $10 \mathrm{deg}$ wide while the mode paths are 22.5 deg apart. The corner boxes have smaller sectors because the modes traveled proportionally less distance in those boxes.

\subsubsection{Solving the inverse problem}

We form a quadratic functional that is sensitive to model estimation error and model smoothness given by

$$
L(\mathrm{f})=(\mathrm{Gf}-\mathrm{t})^{T} \mathrm{~W}(\mathrm{Gf}-\mathrm{t})+\lambda_{1} \mathrm{f}^{T} \mathrm{~S}_{1} \mathbf{f}+\lambda_{2} \mathbf{f}^{T} \mathrm{~S}_{2} \mathbf{f}
$$




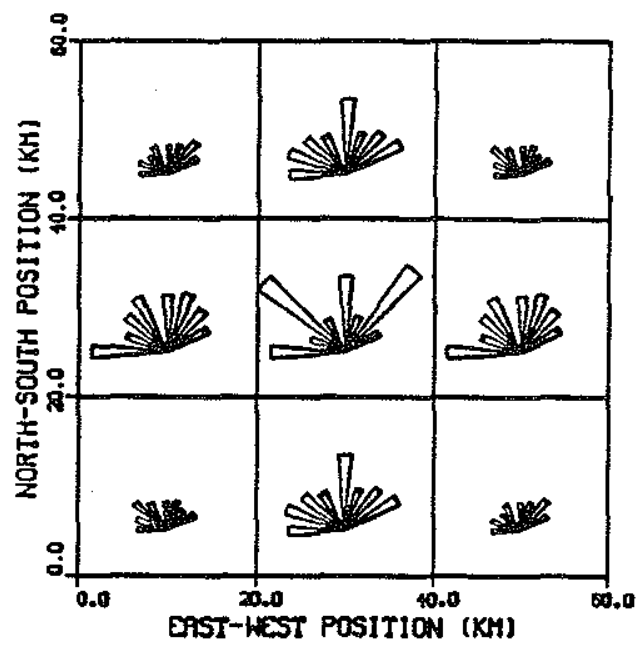

Figure 4.5: Graphical representation of the kernel G.

where $W$ is a weighting matrix which allows us to use different types of measurements with different levels of confidence. The matrices, $S_{1}$ and $S_{2}$, are used to smooth over direction and space, respectively. We describe them in more detail below. The Lagrange multipliers, $\lambda_{1}$ and $\lambda_{2}$, determine how much smoothing is introduced in direction and space. Assuming here that they are known, we will describe the procedure for determining them further on.

We now derive the vector $f$ which minimizes $L$. First, we expand Eq. (4.184) (following Liebelt[63]) as

$$
\begin{gathered}
L(\mathrm{f})=\left(\mathrm{f}^{T} \mathrm{G}^{T}-\mathrm{t}^{T}\right) \mathrm{W}(\mathrm{Gf}-\mathrm{t})+\lambda_{1} \mathrm{f}^{T} \mathrm{~S}_{1} \mathrm{f}+\lambda_{2} \mathrm{f}^{T} \mathrm{~S}_{2} \mathrm{f} \\
L(\mathrm{f})=\mathrm{f}^{T} \mathrm{G}^{T} \mathrm{WG} f-\mathrm{f}^{T} \mathrm{G}^{T} \mathrm{Wt}-\mathrm{t}^{T} \mathrm{WGf}+\mathrm{t}^{T} \mathrm{Wt}+\lambda_{1} \mathrm{f}^{T} \mathrm{~S}_{1} \mathrm{f}+\lambda_{2} \mathrm{f}^{T} \mathrm{~S}_{2} \mathrm{f}
\end{gathered}
$$

Because the third term in the preceding equation is a scalar, we can transpose this term to obtain

$$
L(\mathrm{f})=\mathrm{f}^{T} \mathrm{G}^{T} \mathrm{WGf}-2 \mathrm{f}^{T} \mathrm{G}^{T} \mathrm{Wt}+\mathrm{t}^{T} \mathrm{Wt}+\lambda_{1} \mathrm{f}^{T} \mathrm{~S}_{1} \mathrm{f}+\lambda_{2} \mathrm{f}^{T} \mathrm{~S}_{2} \mathrm{f}
$$


We next differentiate Eq. (4.189) with respect to the components of $f$ yielding

$$
\frac{d L}{d \mathbf{f}}=2 \mathrm{G}^{T} \mathrm{WGf}-2 \mathrm{G}^{T} \mathrm{WT}+2 \lambda_{1} \mathrm{~S}_{1} \mathbf{f}+2 \lambda_{2} \mathrm{~S}_{2} \mathbf{f} .
$$

Setting the expression above to zero and solving for $f$ we get

$$
\mathbf{f}=\frac{\Omega^{2}}{2 \pi d_{1}^{2} g}\left(G^{T} \mathrm{WG}+\lambda_{1} \mathrm{~S}_{1}+\lambda_{2} \mathrm{~S}_{2}\right)^{-1} \mathrm{G}^{T} \mathrm{Wt} .
$$

The solution given in Eq. (4.191) is analogous to the weighted damped least squares solution $[65,66,67,68]$.

We have chosen to break our unknown frequency-direction spectrum model $F(\vec{r}, \Omega, \alpha)$ into a discrete vector $\mathrm{f}$. Since frequency $\Omega$ is only a multiplicative factor in the kernel $\mathrm{G}$, we need only compute one generalized inverse operator $\mathrm{G}_{g}^{-1}$ from Eq. (4.191) i.e.

$$
\mathrm{G}_{g}^{-1}=\frac{\Omega^{2}}{2 \pi d_{1}^{2} g}\left(\mathrm{G}^{T} \mathrm{WG}+\lambda_{1} \mathrm{~S}_{1}+\lambda_{2} \mathrm{~S}_{2}\right)^{-1} \mathrm{G}^{T} \mathrm{~W} .
$$

We thus perform one $\mathrm{NxN}$ inverse operation for all frequencies desired.

\subsubsection{Smoothing Matrices}

Smoothing matrices were introduced into Eq. (4.192) to stabilize the estimate of the unknown spatially dependent frequency-direction spectrum vector $f$. If the matrices were absent, there are an infinite number of solutions, i.e. no unique solution exists for our underdetermined problem.

The smoothing matrix is $S_{1}=D_{1}^{T} D_{1}$ where $D_{1}$ is a matrix which contains the finite difference representation of some norm of our solution. To minimize the second derivative of $\mathrm{f}$ over direction $\alpha$, which gives $D_{i i} \propto-2$ and $D_{i j} \propto 1$ wherever $\mathrm{i}$ and $\mathrm{j}$ represent neighboring directions in $\mathrm{f}$. So then the finite difference representation of our second derivative is

$$
\left.\frac{d^{2} f}{d \alpha^{2}}\right|_{\alpha_{0}} \simeq \frac{f\left(\alpha_{0}-\Delta \alpha\right)-2 f\left(\alpha_{0}\right)+f\left(\alpha_{0}+\Delta \alpha\right)}{\Delta \alpha^{2}}
$$


where $\Delta \alpha$ is the finite difference.

Besides the smoothing done in direction for interpolation, we also must smooth in $(x, y)$ space or, in other words, over boxes. To do this, we introduce a new smoothing matrix $\mathrm{S}_{2}=\mathrm{C}_{2}^{-1}$ where $\mathrm{C}_{2}$ is the correlation matrix for the unknown vector $f$ over the spatial variables $(x, y)$. The values for the correlation matrix entries reflect the fact that physically adjacent boxes should not have very different frequency-direction spectra. For our inversions, we assumed that adjacent boxes had a correlation of .9 between adjacent boxes parallel to shore, and .7 between adjacent boxes perpendicular to the shore. All other boxes had proportionally less correlation based upon distance apart and relation to shore. These correlation values were derived as follows. We calulated a covariance matrix

$$
\mathrm{C}_{2}=E\left[(\mathbf{f}-E(\mathbf{f}))(\mathbf{f}-E(\mathbf{f}))^{T}\right]
$$

The unknowns in the model were the wind speed and direction. The wind speed was assumed to have a Rayleigh distribution with a mean of $10 \mathrm{~m} / \mathrm{s}$. The wind direction was assumed to evenly distributed between 0 and 180 degrees. The correlations were then calculated, giving the results stated above. Moreover, moderate variations in the values of the correlations had very little effect on the inversions.

\subsubsection{Resolution and Variance Tradeoff}

We choose the Lagrange parameters $\lambda_{1}$ and $\lambda_{2}$ used in the generalized inverse in Eq. (4.192) based on the tradeoff between resolution and variance of our unknown frequency-direction vector $f$.

The covariance matrix of $f$ characterizes the degree of error amplification in the inversion process and is given by

$$
[\operatorname{covf}]=\mathrm{G}_{g}^{-1} \mathrm{G}_{g}^{-1 T}
$$


for uncorrelated data [69]. The size of the covariance is often computed as the sum of the diagonal terms of the matrix as

$$
\operatorname{size}([\operatorname{covf}])=\sum_{i=1}^{N}[\operatorname{covf}]_{i i}
$$

The resolution matrix characterizes whether the components in $f$ can be independently resolved, and is given by

$$
\mathbf{R}=\mathbf{G}_{g}^{-1} \mathrm{G} .
$$

The ideal case is that each component of $f$ would be resolved perfectly, i.e. when $\mathrm{G}_{g}=\mathrm{G}$ in which case the resolution matrix would be the identity matrix. A measure of the goodness of the resolution spread is

$$
\operatorname{spread}(\mathbf{R})=\sum_{i=1}^{N} \sum_{j=1}^{N}\left[R_{i j}-I_{i j}\right]^{2} .
$$

The resolution spread and size of the covariance matrix can be plotted as a function of one of the Lagrange multipliers as shown in Fig. 4.6. Varying $\lambda_{1}$ corresponds to moving up and down the curve giving a whole set of solutions. At one extreme, the solution has large variance and the system has high resolution. At the other extreme, the opposite occurs. There is a range of good choices for $\lambda_{1}$, in which the resolution is adequate while the variance is tolerable. For our case, there is another Lagrange multiplier $\lambda_{2}$ we must vary, so the tradeoff curve is actually a tradeoff surface in $\left(\lambda_{1}, \lambda_{2}\right)$ space, but the analysis is the same. For each of the different inversions performed, the parameters were varied until acceptable resolution and variance were obtained

Because the inversions were performed on synthetic data, we have the true frequency-direction spectra model. We are able to compute the total mean squared error between the estimate and the true model. Once acceptable resolution and variance were obtained, variations in $\lambda_{1}$ and $\lambda_{2}$ of an order of magnitude either 


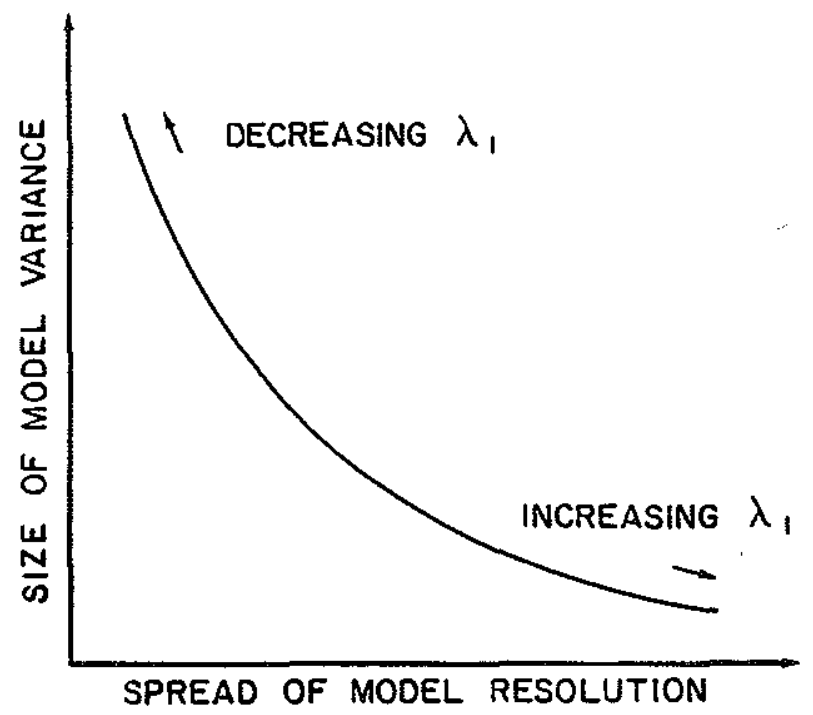

Figure 4.6: Tradeoff curve between resolution and variance.

way in each parameter increased the total squared error by about $20 \%$ in both the noise-free and noisy-ray cases. Therefore, the inverse solution is basically robust to the exact choice of the parameters. Resolution and variance results for specific inversions are presented in Section 4.3.

\subsubsection{Mode Inversion Results: No Noise Case}

A spatially varying frequency-direction spectrum is difficult to present. Therefore, we present results of the inversions in three different formats for both modes and rays. Each format brings out different aspects of the inversions. The formats are:

1. Contour plots with gray level proportional to the spectral amplitude.

2. Graphs of the spectra versus frequency at a constant direction of $90 \mathrm{deg}$.

3. Graphs of the spectra versus direction at a constant frequency of $0.2 \mathrm{~Hz}$. 


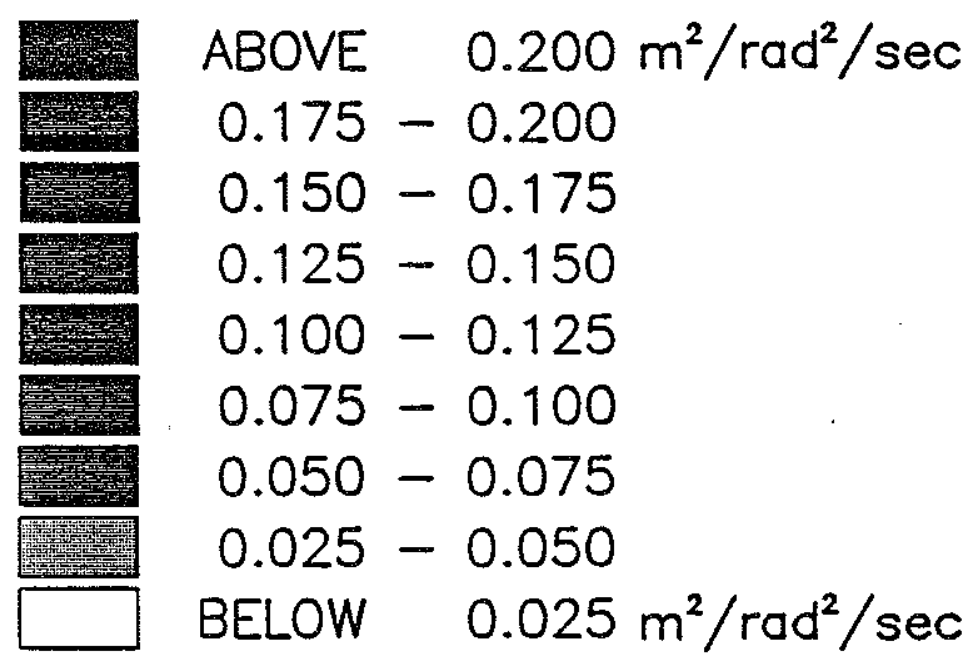

Figure 4.7: Contour plot grey level key.

In this section, we present the results of the inversion of mode travel time perturbation spectra derived from the frequency-direction spectra. That is, we have perfect forward data with no noise. Figure 4.7 shows the grey scale used in all the contour plots in this thesis. Figures $4.8,4.9$, and 4.10 show the results of inversion in the grey level contour plot format. The units of the spectra are $\mathrm{m}^{2} \mathrm{sec} / \mathrm{rad}^{2}$.

Each of the figures present the results for one column of boxes. The rightmost contour plots labelled "ORIGINAL MODEL" show the model (evaluated at the center of each box) used to produce the simulated forward data. The features of the model are evident in the figures. Most of the energy is concentrated in between 60 and $120 \mathrm{deg}$ in direction. The wind is blowing offshore (down the page) with increasing fetch as one goes down the page. The model shows the fetch dependence in amplitude of the spectra, with the furthest fetch spectra rising more than $25 \%$ above the top boxes. Also, the peak frequency goes from $0.25 \mathrm{~Hz}$ in the top boxes to near $0.20 \mathrm{~Hz}$ in the bottom boxes. Because the shore is parallel to the top boxes, there is no change in the model spectra along the rows of boxes, hence the high 


\section{BOX 7}

TOMOGRAPHIC ESTIMATE
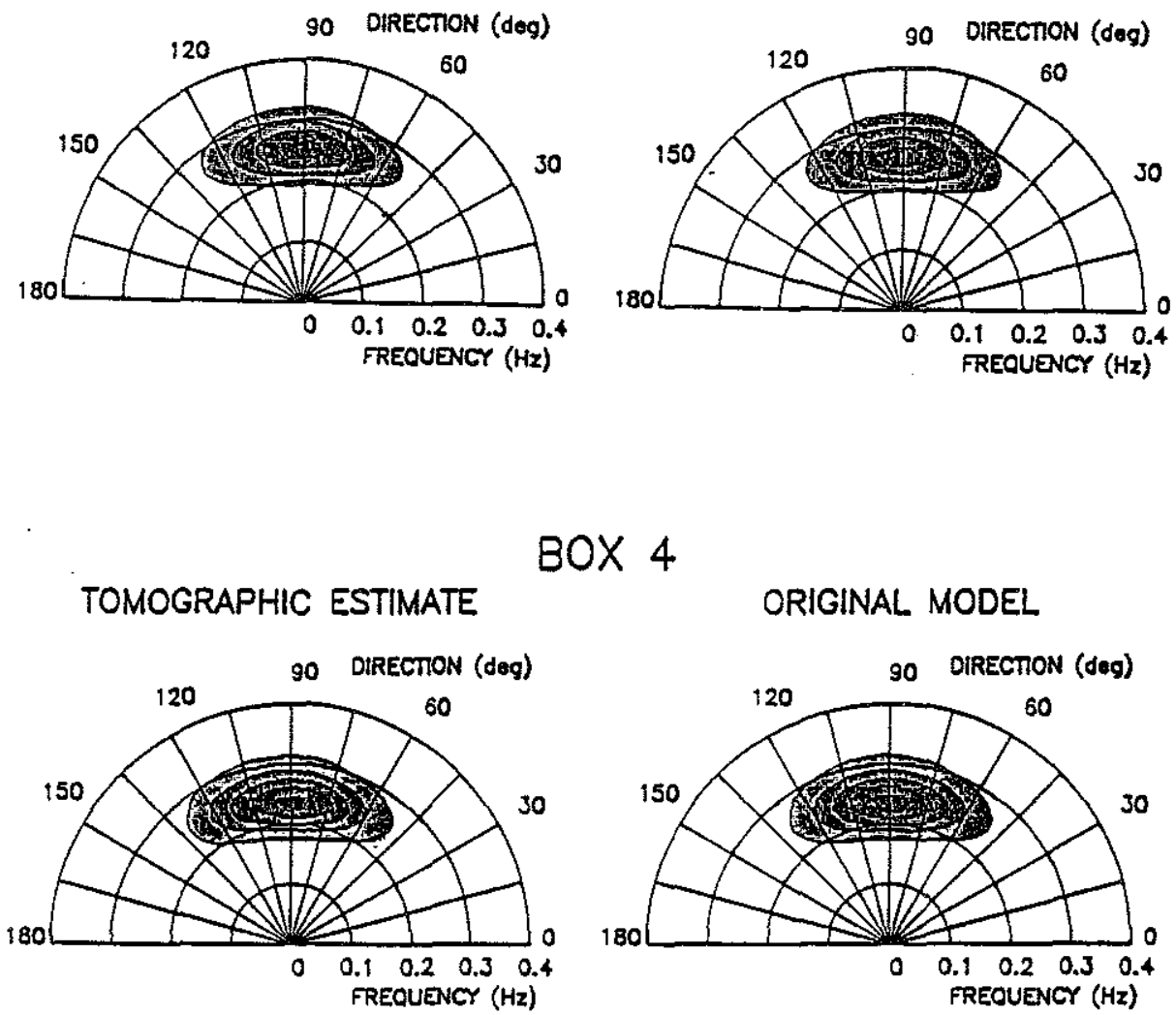

BOX 4
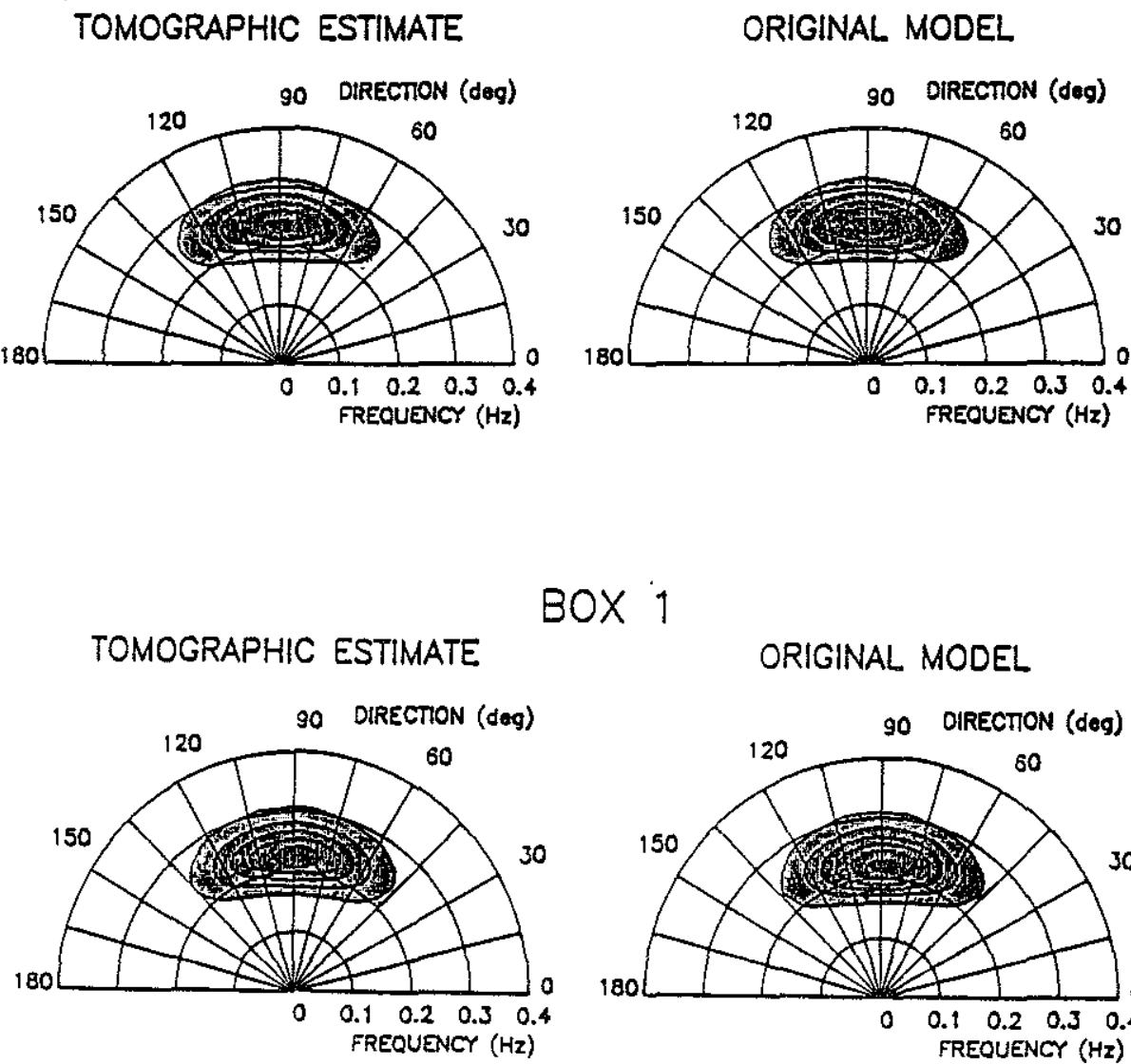

\section{BOX 1}

ORIGINAL MODEL

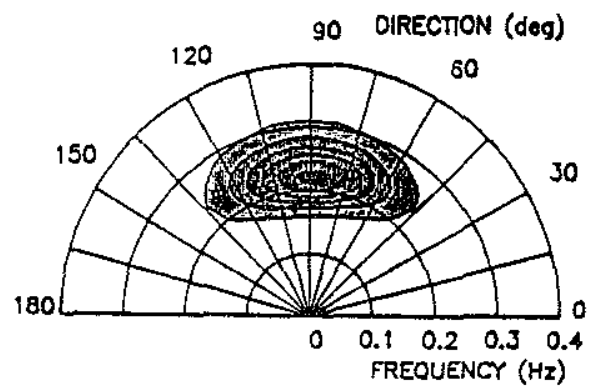

Figure 4.8: Contour plot of inversions of modes with no noise for the frequency-directional spectra in boxes 1,4 , and 7 . See Fig. 4.7 for grey level key. 


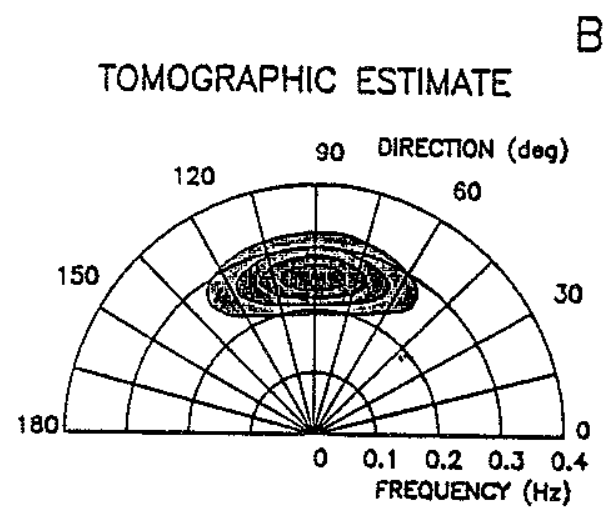

BOX 8
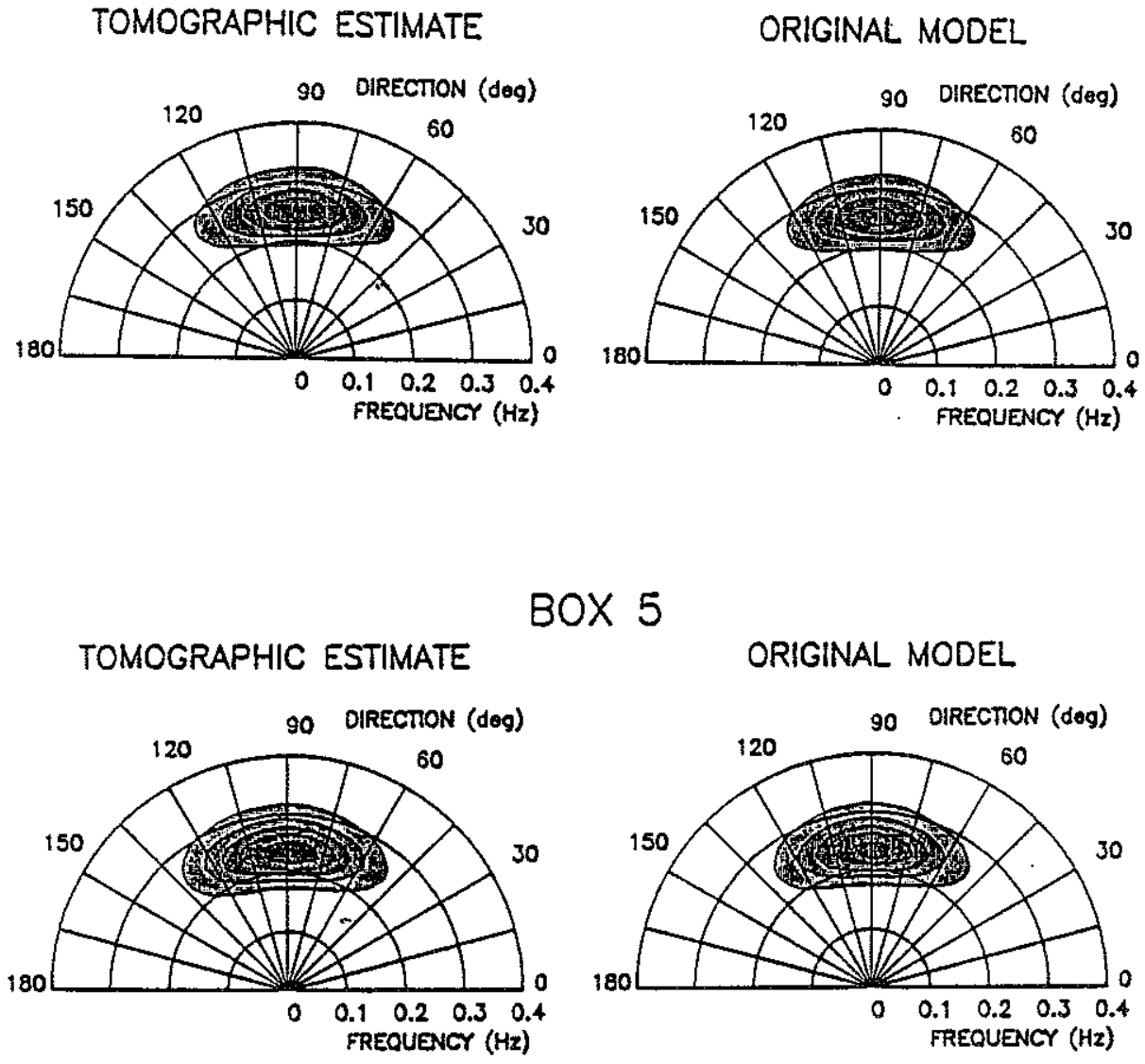

BOX 5
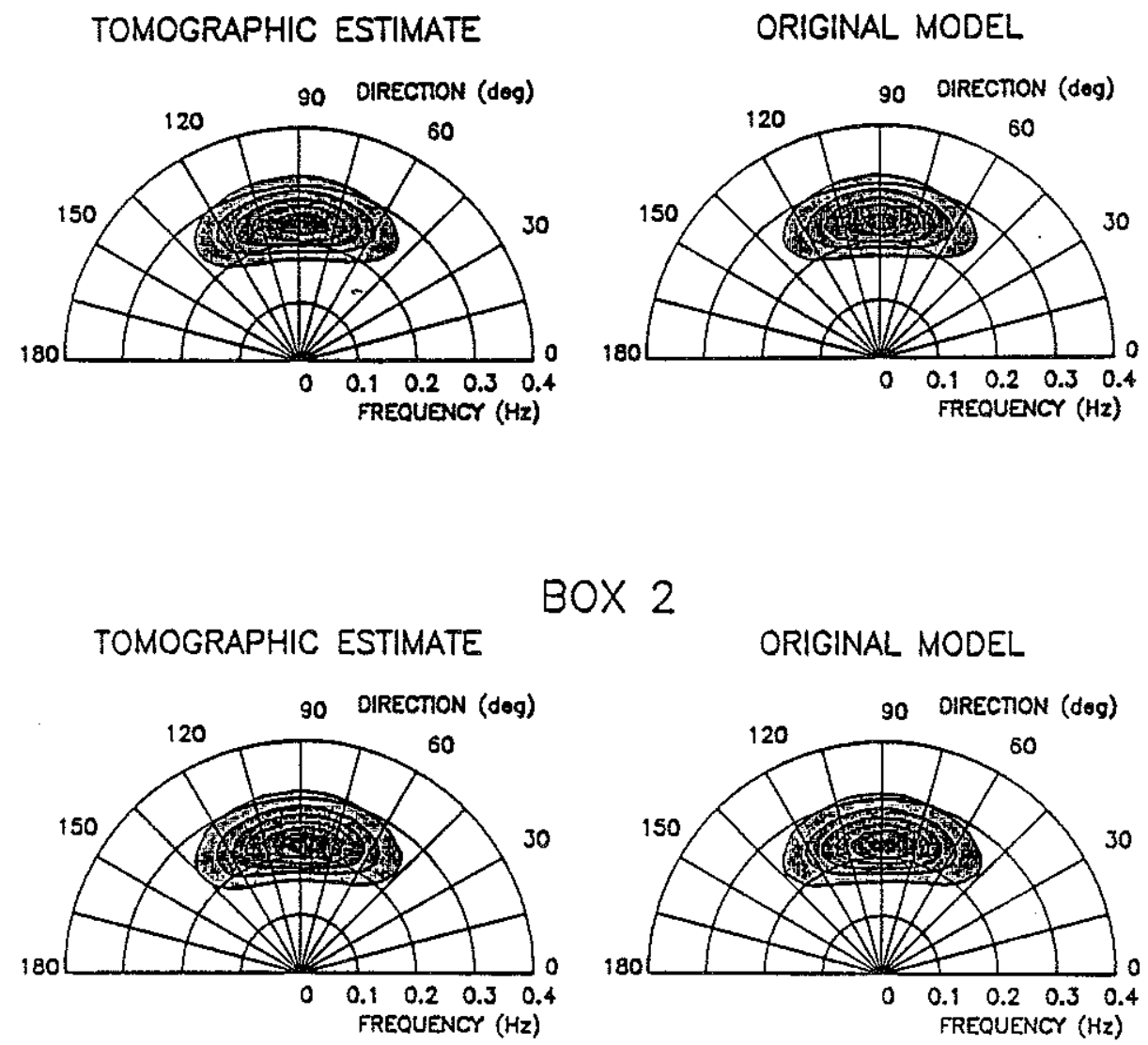

BOX 2

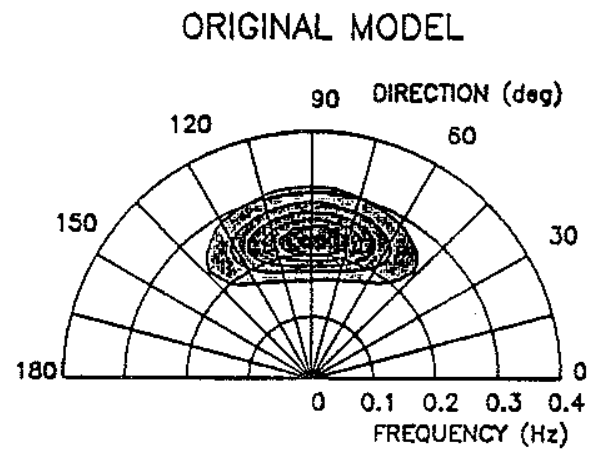

Figure 4.9: Contour plot of inversions of modes with no noise for the frequency-directional spectra in boxes 2,5 , and 8 . See Fig. 4.7 for grey level key. 


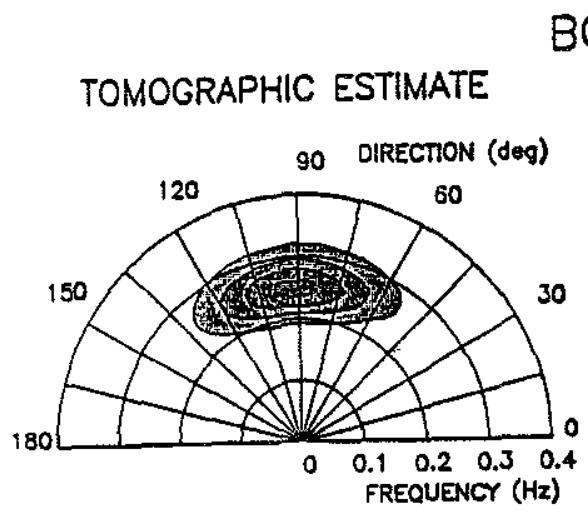

BOX 9

ORIGINAL MODEL

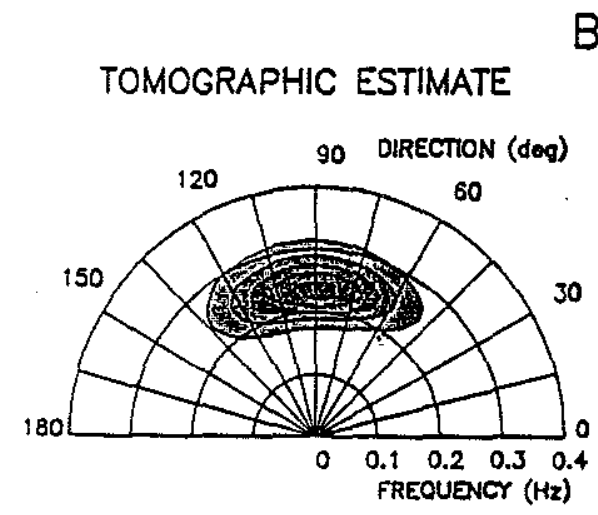

BOX 6
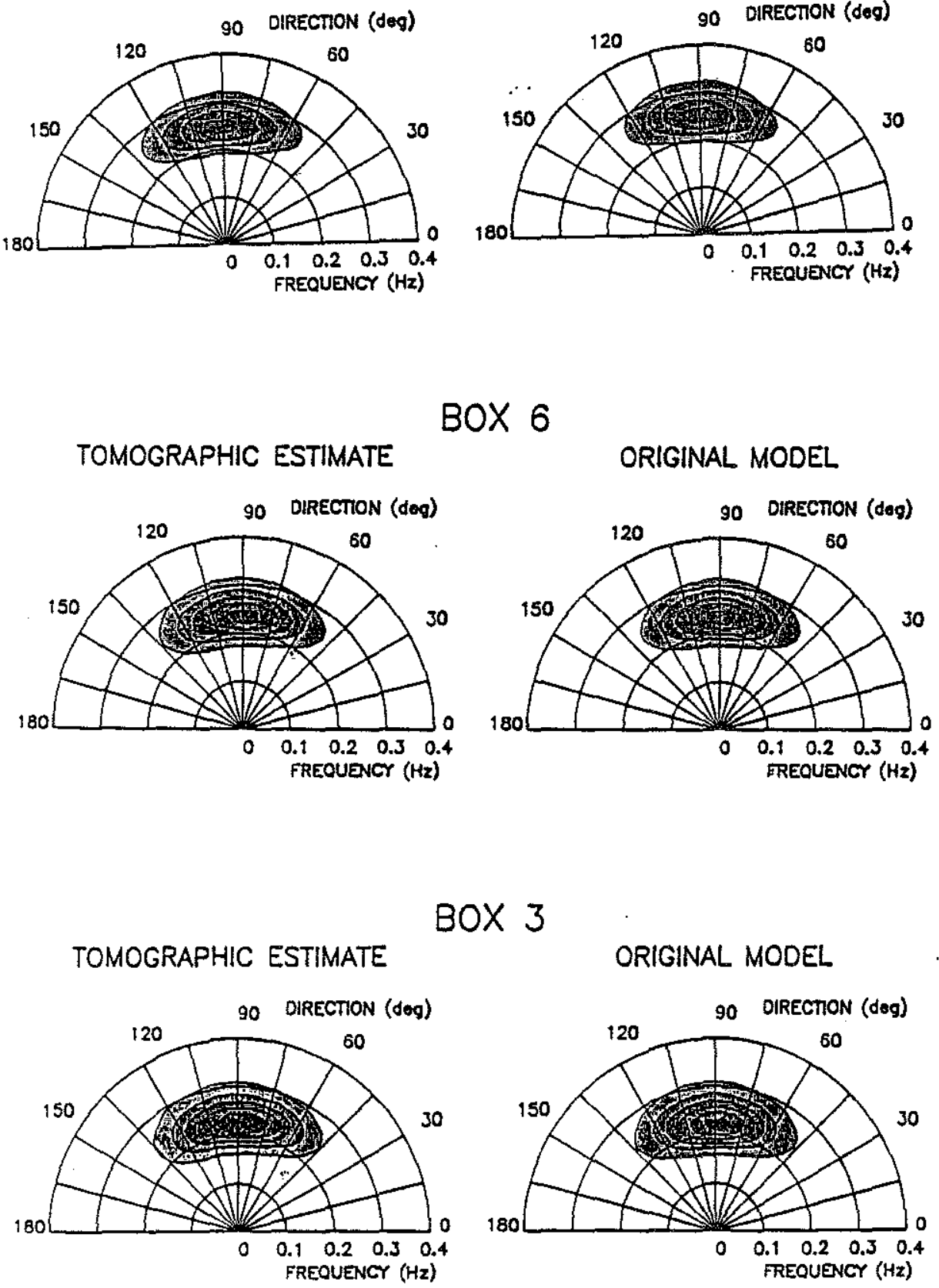

\section{BOX 3}

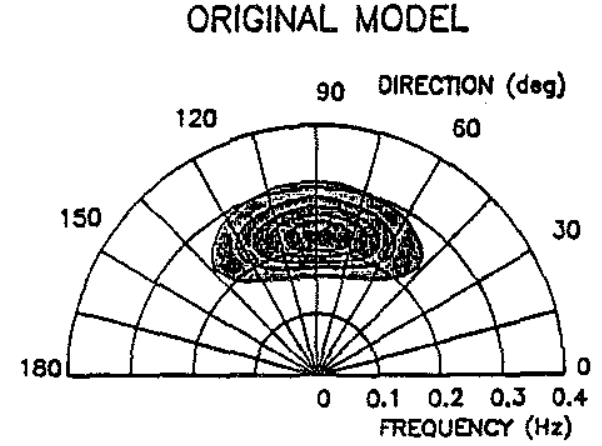

Figure 4.10: Contour plot of inversions of modes with no noise for the frequency-directional spectra in boxes 3,6 , and 9. See Fig. 4.7 for grey level key. 
correlation between shore parallel boxes.

The leftmost contour plots labelled "TOMOGRAPHIC ESTIMATE" in each of the figures show the results of the inversion. The inverted spectra follow the energy of the spectra in direction very well. The location of the peak frequency is tracked between boxes. The amplitudes of the inverted spectra also agree fairly closely with those of the model. The center column of boxes 2,5 , and 8 match the best as shown in Fig. 4.9. We used a smoothing matrix $\mathrm{S}_{2}$ to help in reducing the underdeterminedness of the inversion. This smoothing matrix is least helpful on the corner boxes because the path lengths of the acoustic modes are least in those boxes, and hence, the amplitude of the entries in the kernal matrix G.

The inversion results can also be presented in a more standard y versus $\mathrm{x}$ format. Figure 4.11 shows the inversion-model comparison for the same three columns of boxes as we did for the contour plots. The spectra are plotted versus frequency for a constant direction of $90 \mathrm{deg}$. The smooth line is the original model used to generate the synthetic data. The dots show the estimated spectra from the inversions. The inversions were repeated every $0.01 \mathrm{~Hz}$. The results show that the inversions are very accurate in regions away from the peak frequency, while they are moderately accurate at the peak frequency. The difference may be due to the smoothing matrix $\mathbf{S}_{2}$ which correlates adjoining boxes and prevents abrupt changes in the spectra between boxes.

We also plot the spectra versus direction at a constant frequency of $0.2 \mathrm{~Hz}$ as shown in Fig. 4.12. This type of presentation shows how well the inversions follow the growth of the spectra with fetch. The corner problem is also evident in these plots. The inverted result follows the orignal model in direction very well. 

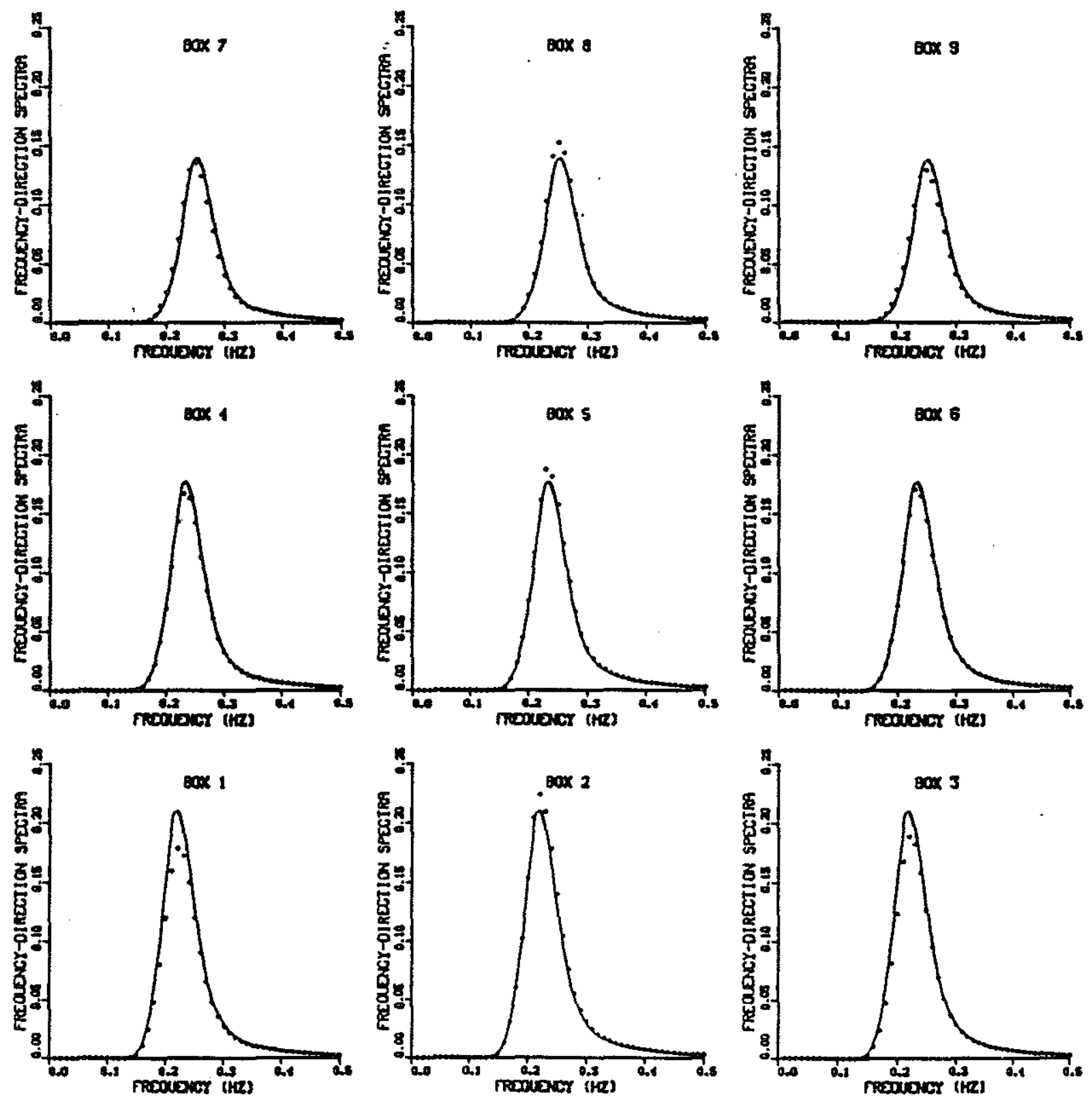

Figure 4.11: Inversion of modes with no noise versus frequency at direction of 90 deg. Inverted data is given by $a \cdot$ while the original model is given by the smooth curve. 

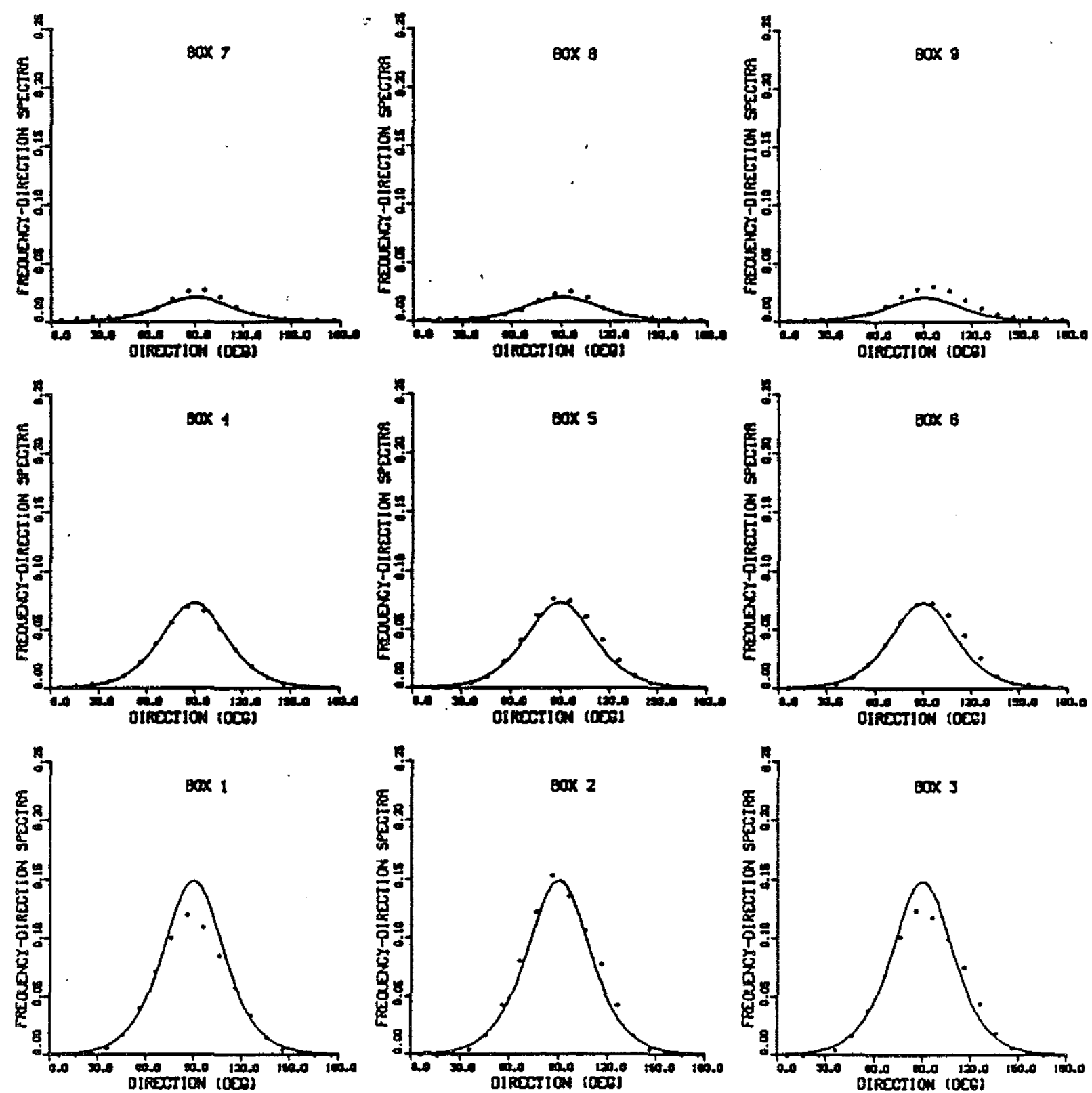

Figure 4.12: Inversion of modes with no noise versus direction at a frequency of 0.2 $\mathrm{Hz}$. Inverted data is given by $\mathrm{a} \bullet$ while the original model is given by the smooth curve. 


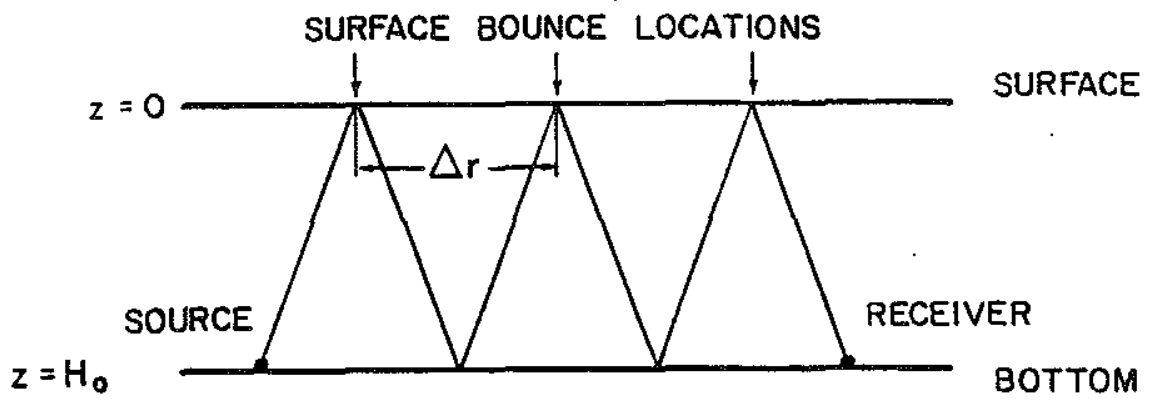

Figure 4.13: Ocean waveguide for inversions.

\subsection{Inversion of Rays}

A ray samples the surface at only discrete points along the path between source and receiver. While we had to discretize the integral relating mode travel time spectra and surface wave spectra, the ray travel time is inherently discrete. For all inversions, we will assume an ocean waveguide as shown in Fig. 4.13. The waveguide depth is $400 \mathrm{~m}$. For convenience in calculations, both the source and receiver are positioned at the bottom of the waveguide. The range between source and receiver depends on the source-receiver pair of interest from Fig. 4.3. In the mode inversions, we only had to use one mode per source-receiver pair because the mode sampled the sea surface continuously. However, now we must use a number of rays per sourcereceiver pair for the inversion to succeed. For most of the inversions with rays, we use six rays per source-receiver pair ranging from rays with 10 surface bounces to 15 surface bounces. This gives us 6 rays multiplied by 28 source-reciever pairs for a total of 168 pieces of data. Remembering that we are estimating 162 unknowns, we 
might assume that we are now overdetermined. But that is not the case. The rays are not completely independent, leaving us with a still underdetermined inversion in $(\mathrm{x}, \mathrm{y})$ space. For comparison, we also performed an inversion on three rays with 13 to 15 surface bounces, giving a total of 84 pieces of data. We studied the sensitivity of the technique to the number of ray bounces by performing an inversion using nine rays with 1 to 9 surface bounces.

In Section 4.2.1, the ray inverse problem is posed in a similiar manner to the mode case. Section 4.2 .2 presents inversion results with noise-free data from six rays. Section 4.2.3 deals with inversions of six rays with the realistic SNR's that are obtained in tomography experiments. Section 4.2 .4 shows the results when the SNR's are $10 \mathrm{~dB}$ less than Section 4.2.3. The effect of only using three rays in the inversion is demonstrated in Section 4.2.6. The inversion results using nine rays with 1 to 9 surface bounces is described in Section 4.2.7.

\subsubsection{Posing the Ray Inverse Problem}

When the surface wave frequency-direction spectrum is spatially inhomogenous, we can generalize Eq. (3.175) in a similar manner to the mode case by

$$
\Theta_{n i j}^{\mathrm{ray}}(\Omega)=\frac{\left(2 k \sin \theta_{n}\right)^{2} 2 \pi g}{\Omega^{2} \Delta \tau} \sum_{m=1}^{M} F\left(\Omega, \alpha_{i j}, r_{m}\right) .
$$

We discretize the frequency-direction spectra $F$ into a vector exactly like Eq. (4.183). However we now have a much longer data vector $t$ made up of the phase perturbation for each source-receiver pair and for ray bounce numbers 10 though 15 for the six ray inversions, 13 through 15 for the three ray inversions, and bounce numbers 1 through 9 for the nine ray inversions. The generalized inverse is found in the exact manner as the mode inverse, i.e.

$$
\mathrm{f}=\frac{\Omega^{2}}{2 \pi\left(2 k \sin \theta_{n}\right)^{2} g}\left(\mathrm{G}^{T} \mathrm{WG}+\lambda_{1} \mathrm{~S}_{1}+\lambda_{2} \mathrm{~S}_{2}\right)^{-1} \mathrm{G}^{T} \mathrm{Wt} .
$$




\subsubsection{Six Ray Inversion Results: No Noise Case}

In this section, we present the results of inversions of ray travel time perturbation spectra synthetically derived from the frequency-direction spectra. That is, we have perfect forward data with no noise. We used six rays between each source and receiver with number of bounces between 10 and 15. Figures 4.14, 4.15, and 4.16 show the results of inversion in the grey level contour plot format. The units of the spectra are again $\mathrm{m}^{2} \mathrm{sec} / \mathrm{rad}^{2}$. The results obtained using six rays are almost indistinguishable from the mode inversions. As the number of rays used in the inversion increases, the quality of results approach that using one mode. As we have described, the mode continuously samples the sea surface while rays sample discretely. For these results, we increased the number of rays in the inversion until they approached that of the modes. The results using three rays per source/receiver pair (bounces 13-15) are shown in Section 4.2.6.

The ray inversion results can also be presented in the standard $\mathrm{y}$ versus $\mathrm{x}$ format. Figure 4.17 shows the inversion-model comparison for the same three columns of boxes as we did for the contour plots. The spectra are plotted versus frequency for a constant direction of $90 \mathrm{deg}$. The smooth line is the original model used to generate the synthetic data. The dots show the estimated spectra from the inversions. The inversions were repeated every $0.01 \mathrm{~Hz}$. These plots show that there is very little difference between the six ray inversions and the mode inversions. We also plot the spectra versus direction at a constant frequency of $0.2 \mathrm{~Hz}$ as shown in Fig. 4.18.

\subsubsection{Six Ray Inversion Results: Noisy Data}

Spectral noise is defined here as the power spectrum of the error in the travel time or phase estimates incurred when we have a finite acoustic SNR. Given the SNR we can compute the standard deviation of the travel time and phase estimates 


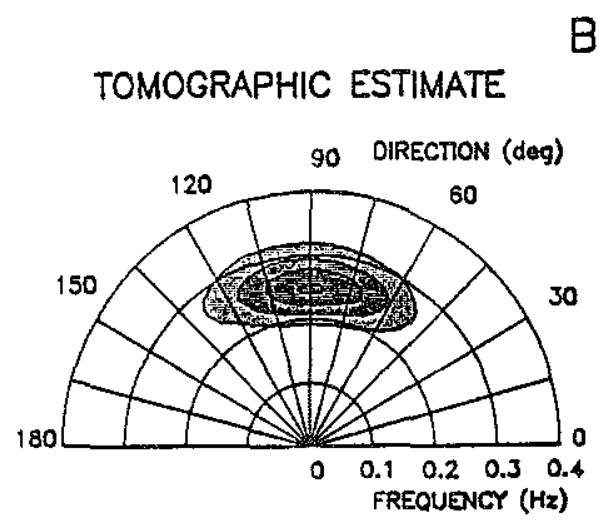

\section{BOX 7}

ORIGINAL MODEL

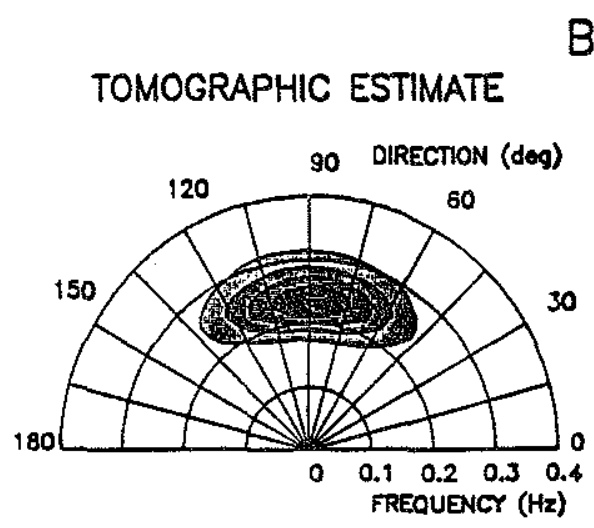

BOX 4
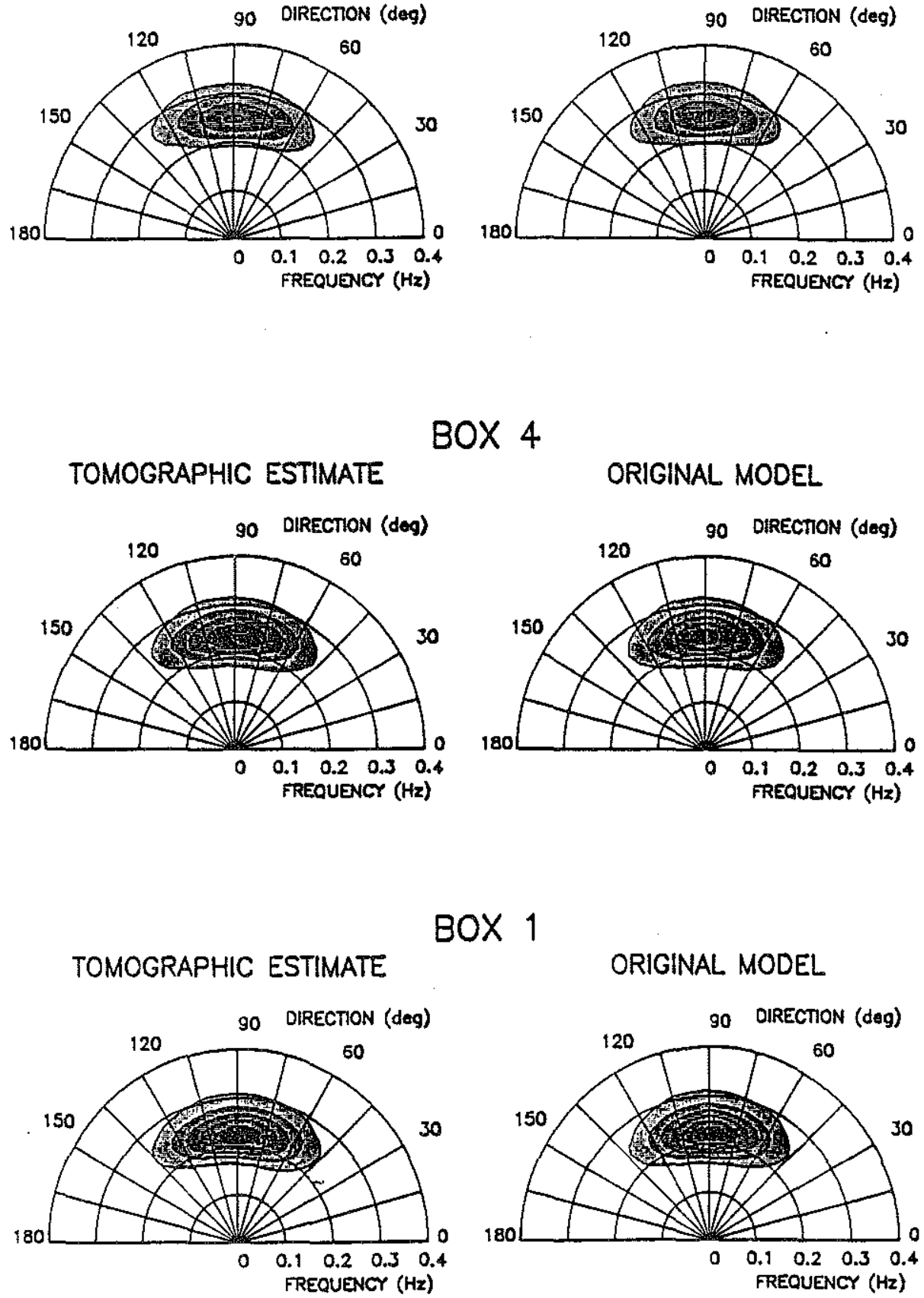

BOX 1
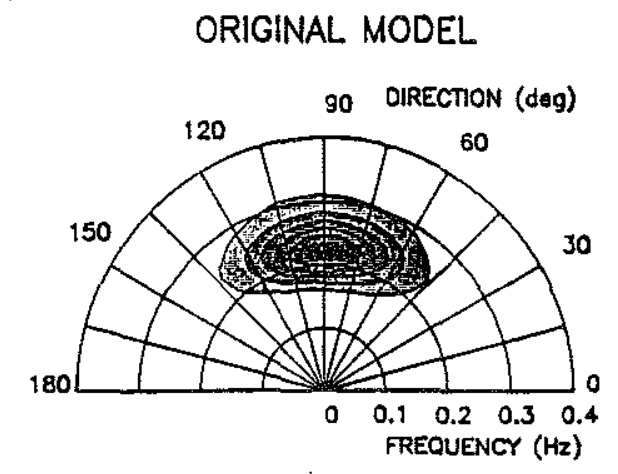

Figure 4.14: Contour plot of inversions of six rays (bounces 10-15) with no noise for the frequency-directional spectra in boxes 1,4 , and 7 . See Fig. 4.7 for grey level key. 


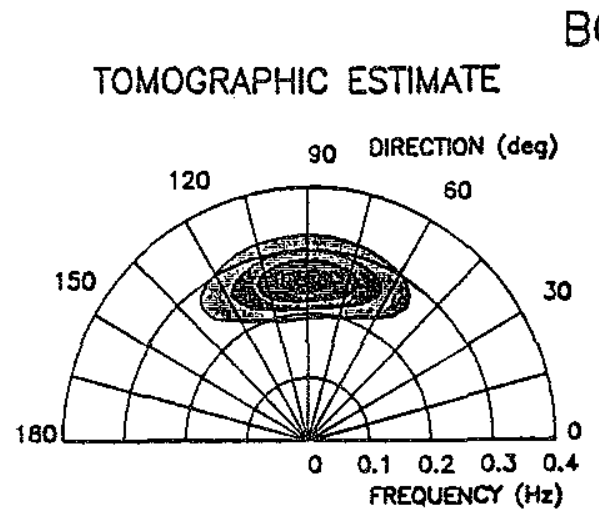

BOX 8
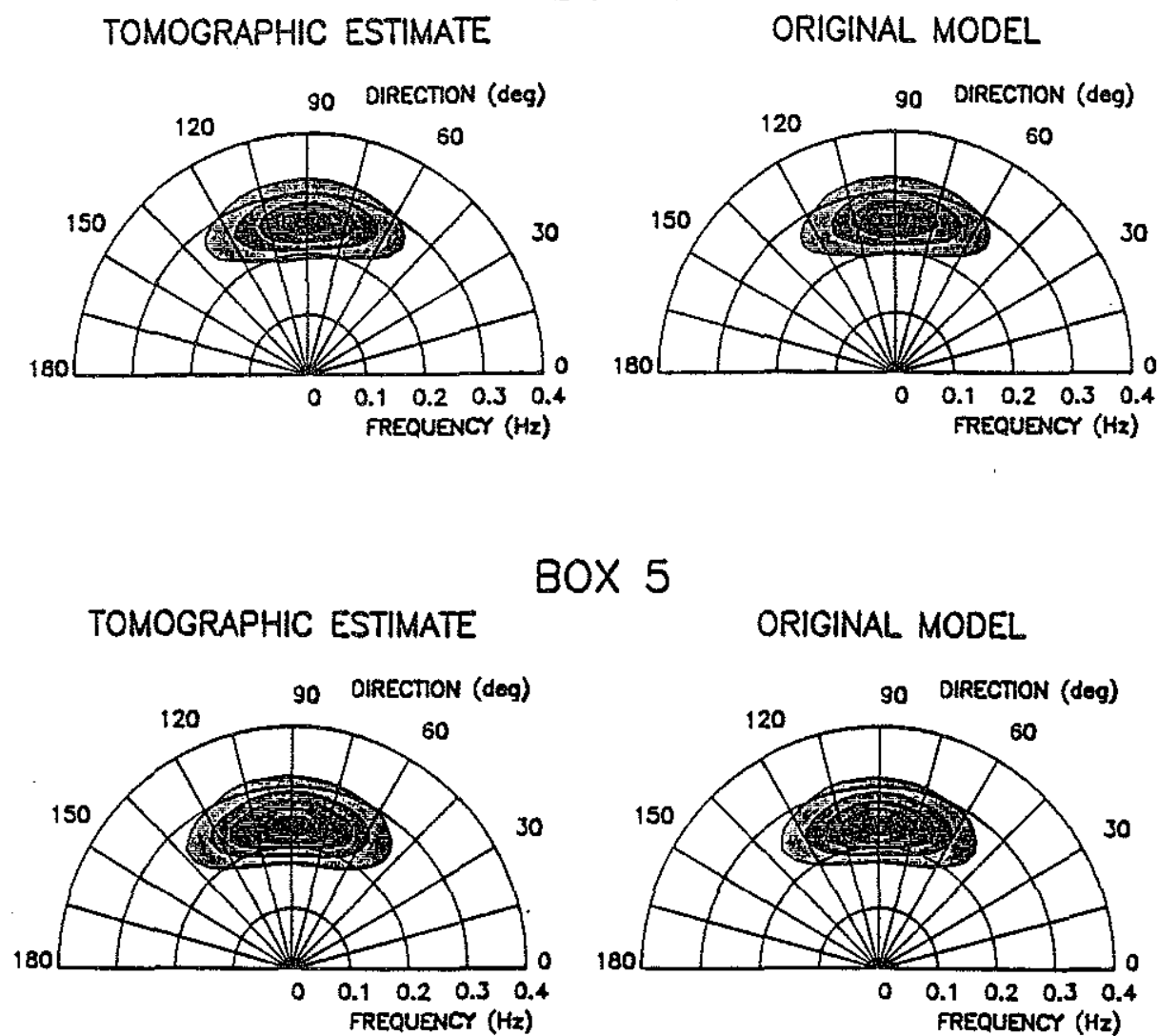

BOX 5
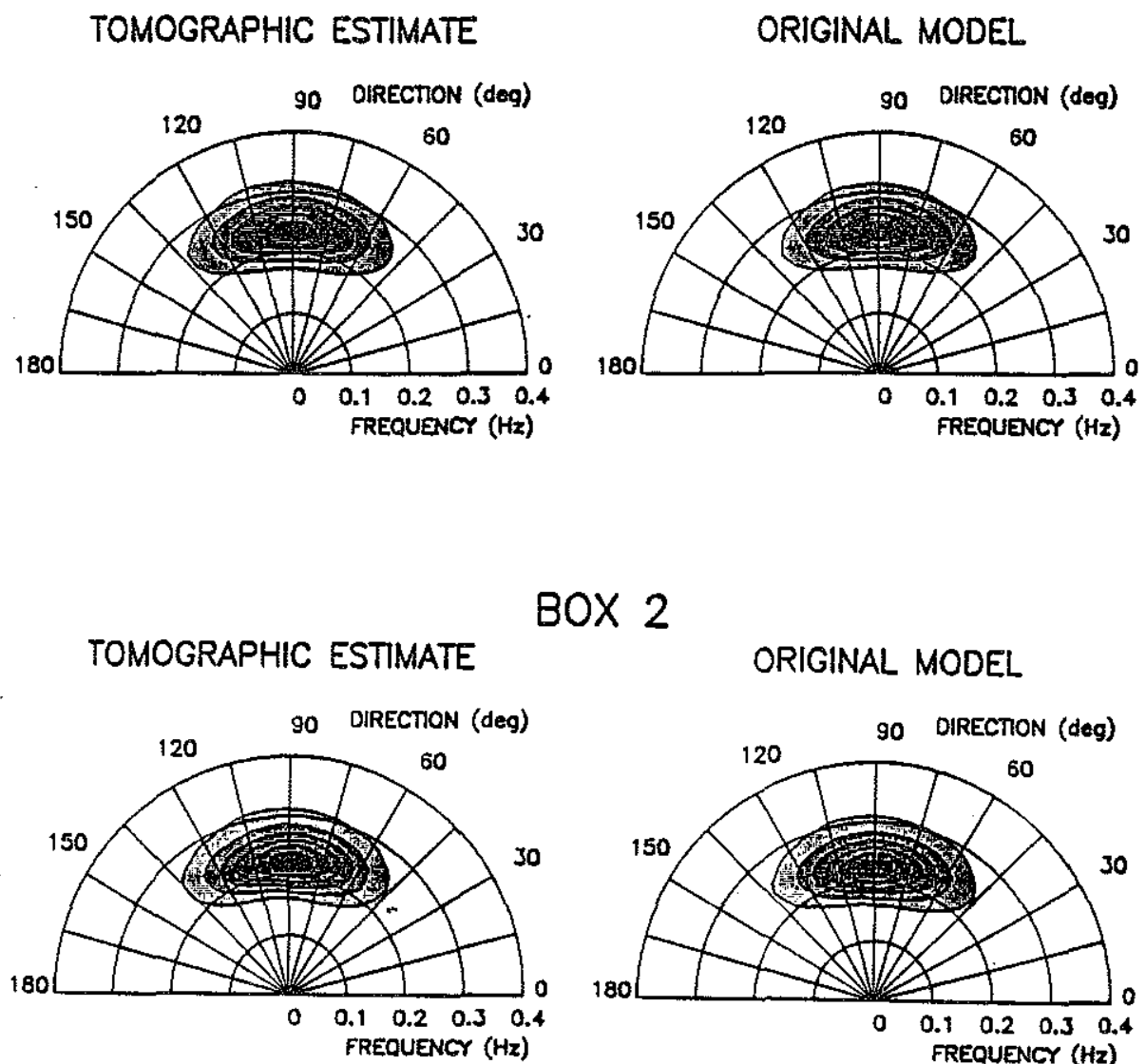

BOX 2

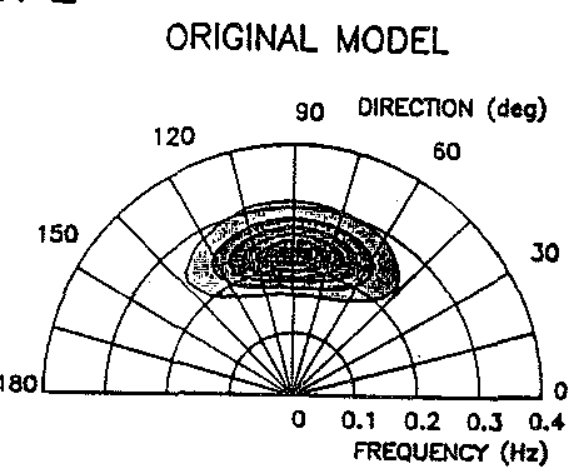

Figure 4.15: Contour plot of inversions of six rays (bounces 10-15) with no noise for the frequency-directional spectra in boxes 2,5 , and 8 . See Fig. 4.7 for grey level key. 


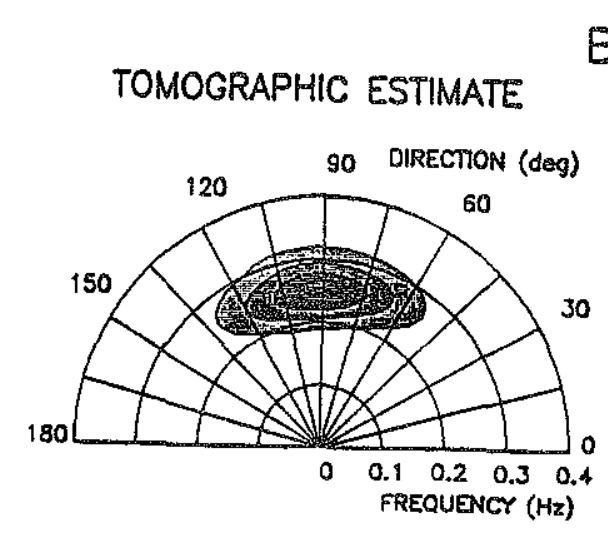

BOX 9
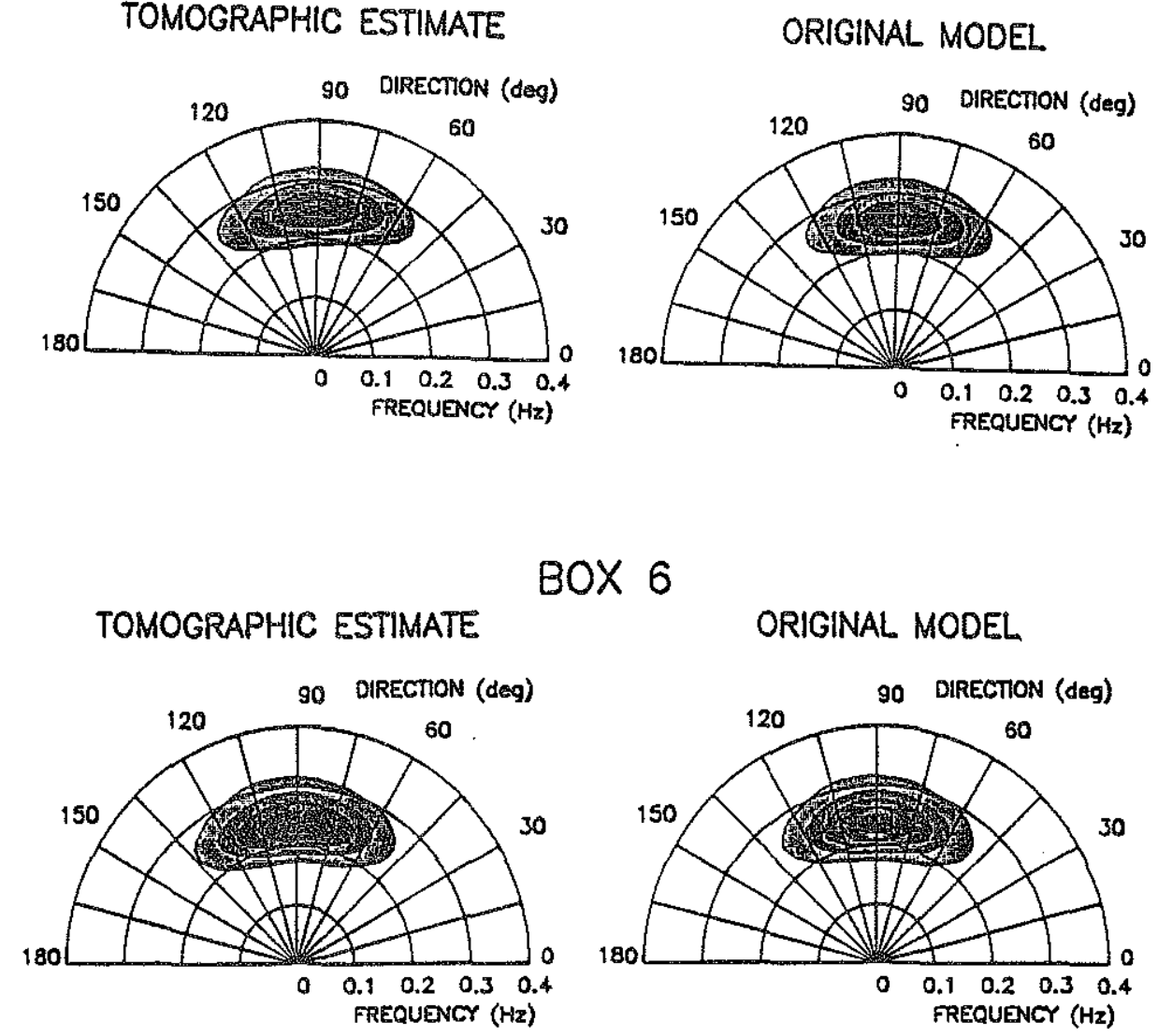

BOX 6
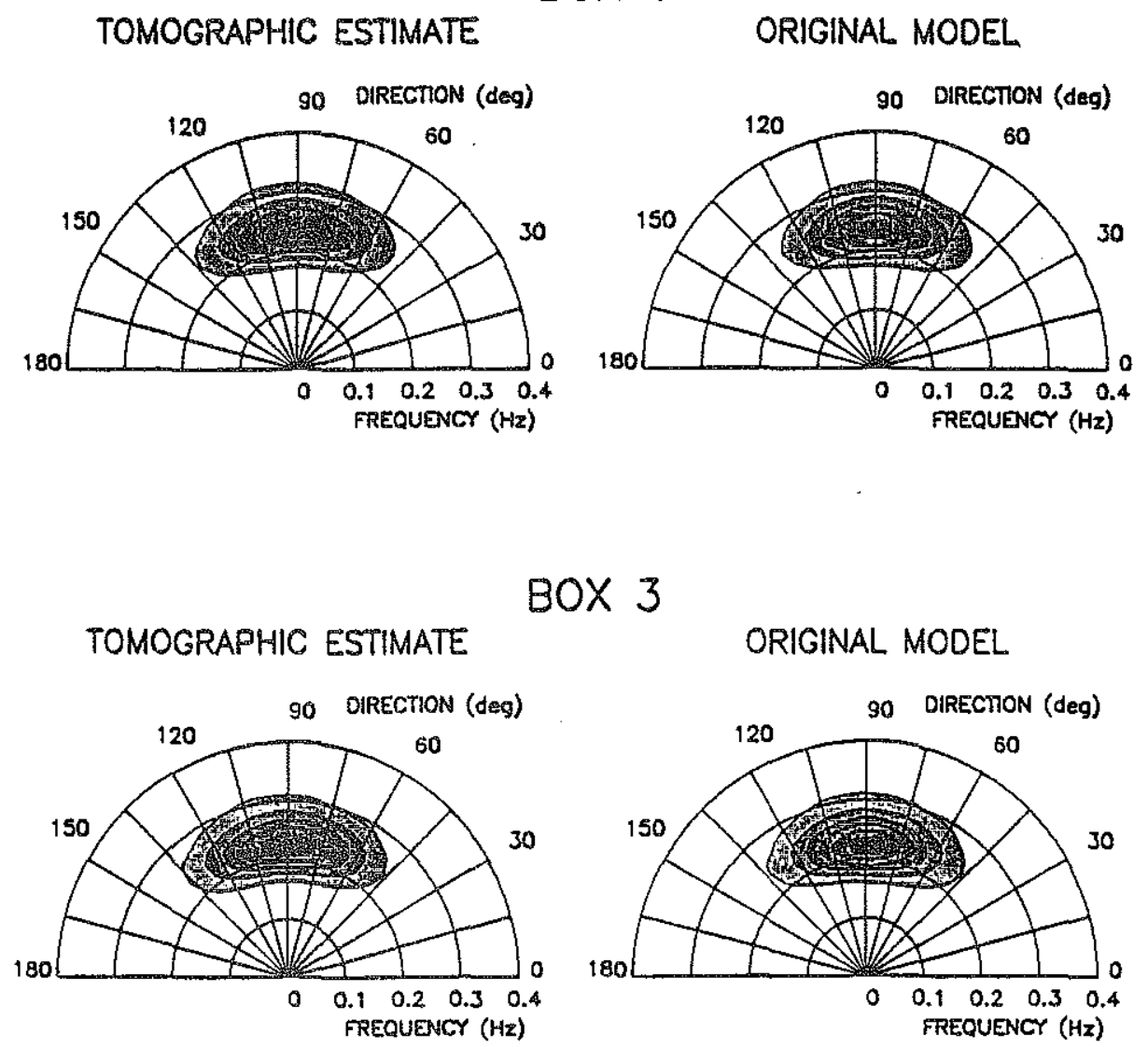

\section{BOX 3}

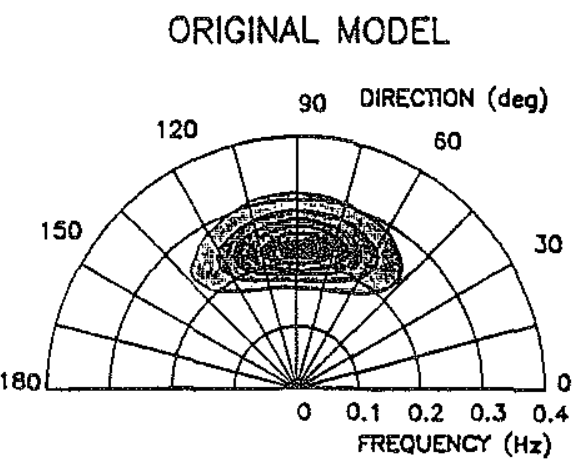

Figure 4.16: Contour plot of inversions of six rays (bounces 10-15) with no noise for the frequency-directional spectra in boxes 3,6 , and 9 . See Fig. 4.7 for grey level key. 

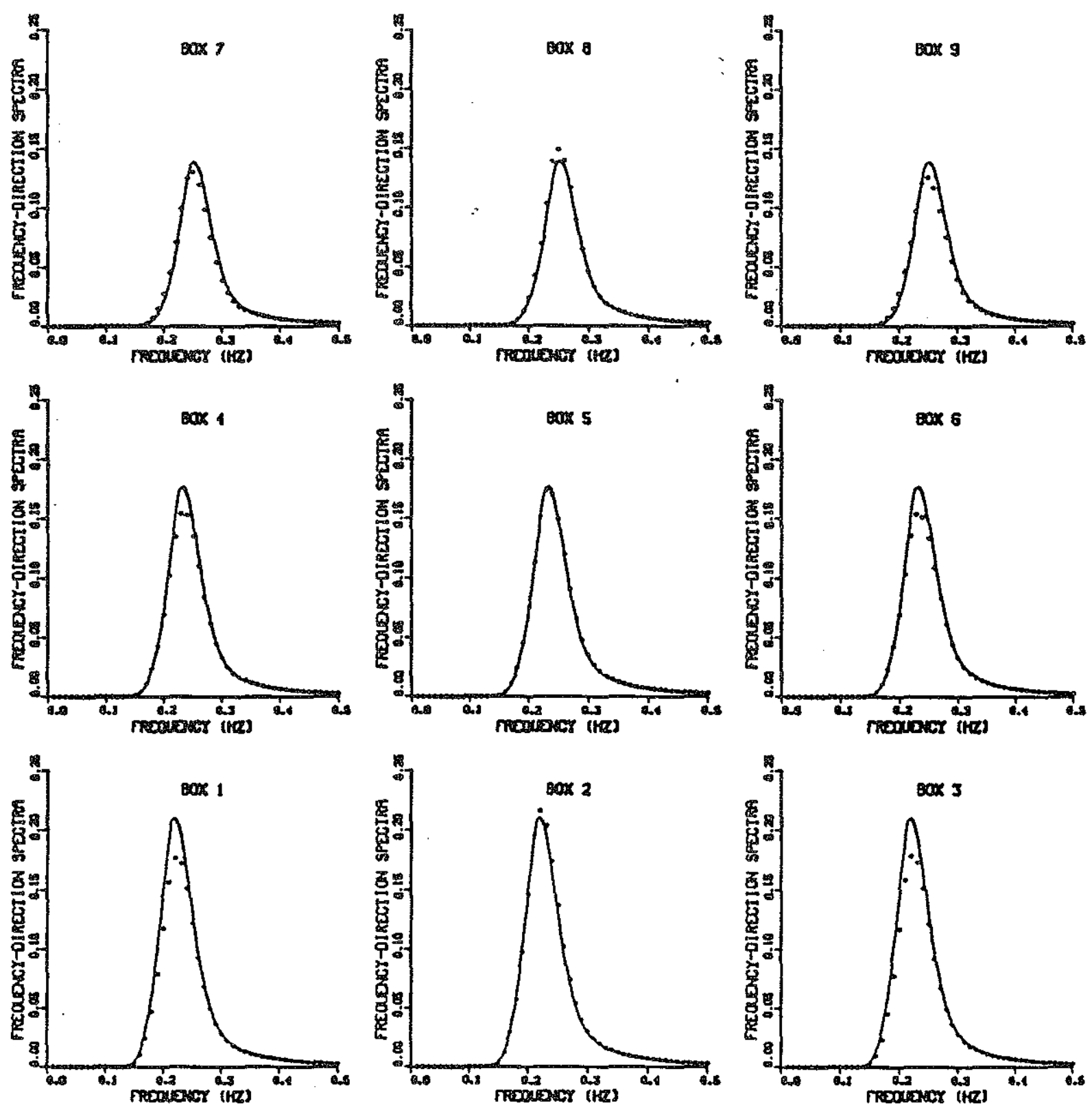

Figure 4.17: Inversion of six rays (bounces 10-15) with no noise versus frequency at direction of $90 \mathrm{deg}$. Inverted data is given by a while the original model is given by the smooth curve. 

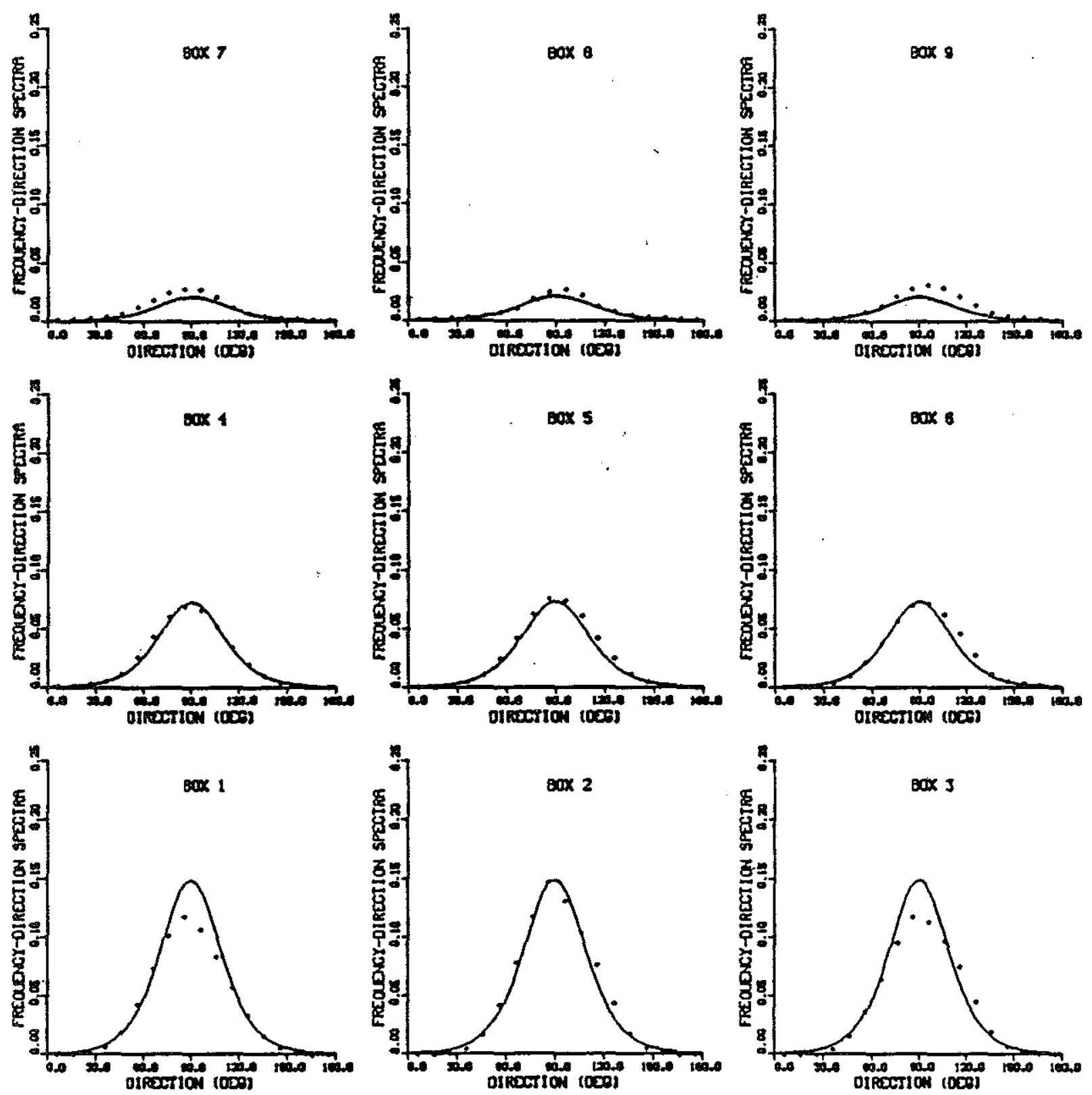

Figure 4.18: Inversion of six rays (bounces 10-15) with no noise versus direction at a frequency of $0.2 \mathrm{~Hz}$. Inverted data is given by a - while the original model is given by the smooth curve. 
by $[4]$

$$
\begin{gathered}
\sigma_{\tau}=\frac{1}{2 \pi \Delta f \sqrt{\mathrm{SNR}}} \\
\sigma_{\varphi}=2 \pi f \sigma_{\tau}=\frac{f}{\Delta f \sqrt{\mathrm{SNR}}}
\end{gathered}
$$

where $f$ and $\Delta f$ are the center frequency and bandwidth of the acoustic signal. We model the spectrum of these errors as white so essentially a.constant in frequency is added onto the phase or travel time spectra.

We now calculate the SNR. The transmission loss of a ray (for our case of bottom-mounted tranceivers) due to spreading is given by Tindle and Bold [70] as

$$
\mathrm{TL}_{1}=\frac{1}{R \sqrt{1+\left(\frac{2 n H}{R}\right)^{2}}}
$$

where $R$ is the range between source and receiver, $H$ is the height of the waveguide, and $n$ is the number of ray bounces. The loss due to the surface scattering is given by $[40]$

$$
\mathrm{TL}_{2}=\left(1-\frac{P^{2}}{2}\right)^{n}
$$

where $P=2 k \sigma \sin \theta_{n}$ is the Rayleigh parameter, $\theta_{n}$ is the incidence angle of the $n^{\text {th }}$ ray and $\sigma$ is the standard deviation of the surface height. For example, for $P=.2$, $n=15, H=400 \mathrm{~m}$, and $R=50 \mathrm{~km}$, we get

$$
\mathrm{TL}=\mathrm{TL}_{1}+\mathrm{TL}_{2}=-48.4 \mathrm{~dB}
$$

The source level SL assumed here is $185 \mathrm{~dB}$, which is typical of tomographic sources[4]. We will assume an acoustic noise loss of $-81 \mathrm{~dB}$ and attenuation due to other sources such as volume effects, bottom effects, etc. of $-5 \mathrm{~dB}$. The attenuation loss used here is very rough, but for the ranges discussed in this thesis (10 $-100 \mathrm{~km}),-5 \mathrm{~dB}$ is probably ballpark for totally internally reflected rays. The resulting calculation becomes 


\begin{tabular}{||l|l||}
\hline SL & $185 \mathrm{~dB}$ \\
$\mathrm{TL}$ & -48 \\
Atten & -5 \\
Noise & -81 \\
\hline SNR & $50 \mathrm{~dB}$ \\
\hline
\end{tabular}

For a transmitting frequency of $220 \mathrm{~Hz}$ and bandwidth of $12 \mathrm{~Hz}$ typical of Webb Research Corp. organ pipe sources [4], the phase variance can be calculated using Eq. (4.202) to be $11 \mathrm{deg}^{2}$.

For each ray in our simulation, a different SNR is calculated and the appropriate white noise is added to the phase spectrum due to the surface waves. Note that Eq. (4.200) contains a multiplication by $\Omega^{2}$. Because of this factor, any flat phase spectrum (like white noise) will be affected at higher frequencies much more than low frequencies. The phase estimation error will be transformed into a steadily increasing noise floor in the surface wave frequency-direction spectrum. Using the values assumed above, we performed an inversion on this "noisy" data. The results are shown in Figs. 4.19, 4.20, and 4.21 in the contour plot format, in Fig. 4.22 as plots versus frequency, and in Fig. 4.23 as plots versus direction. Comparing these plots to their no noise counterparts, we can see small differences at high frequencies as we expected. Note that this is just where we may be limited by the Nyquist criteria anyway. We cannot send signals too often due to the multipath arrival structure spread. But noise can also limit surface wave tomography at high frequencies, making this the most difficult part of the spectrum for our technique to work in. 


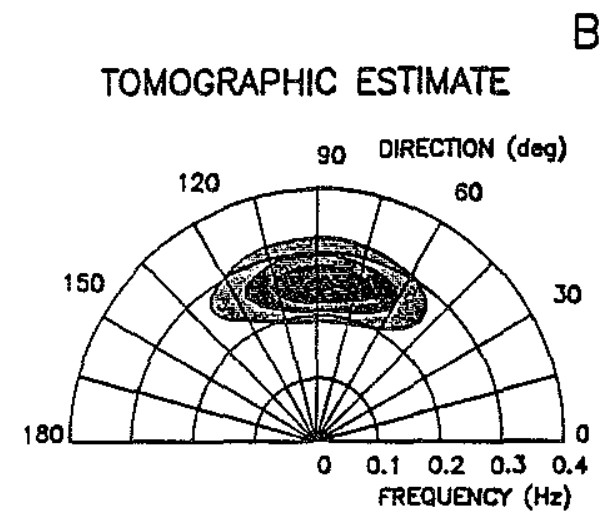

BOX 7
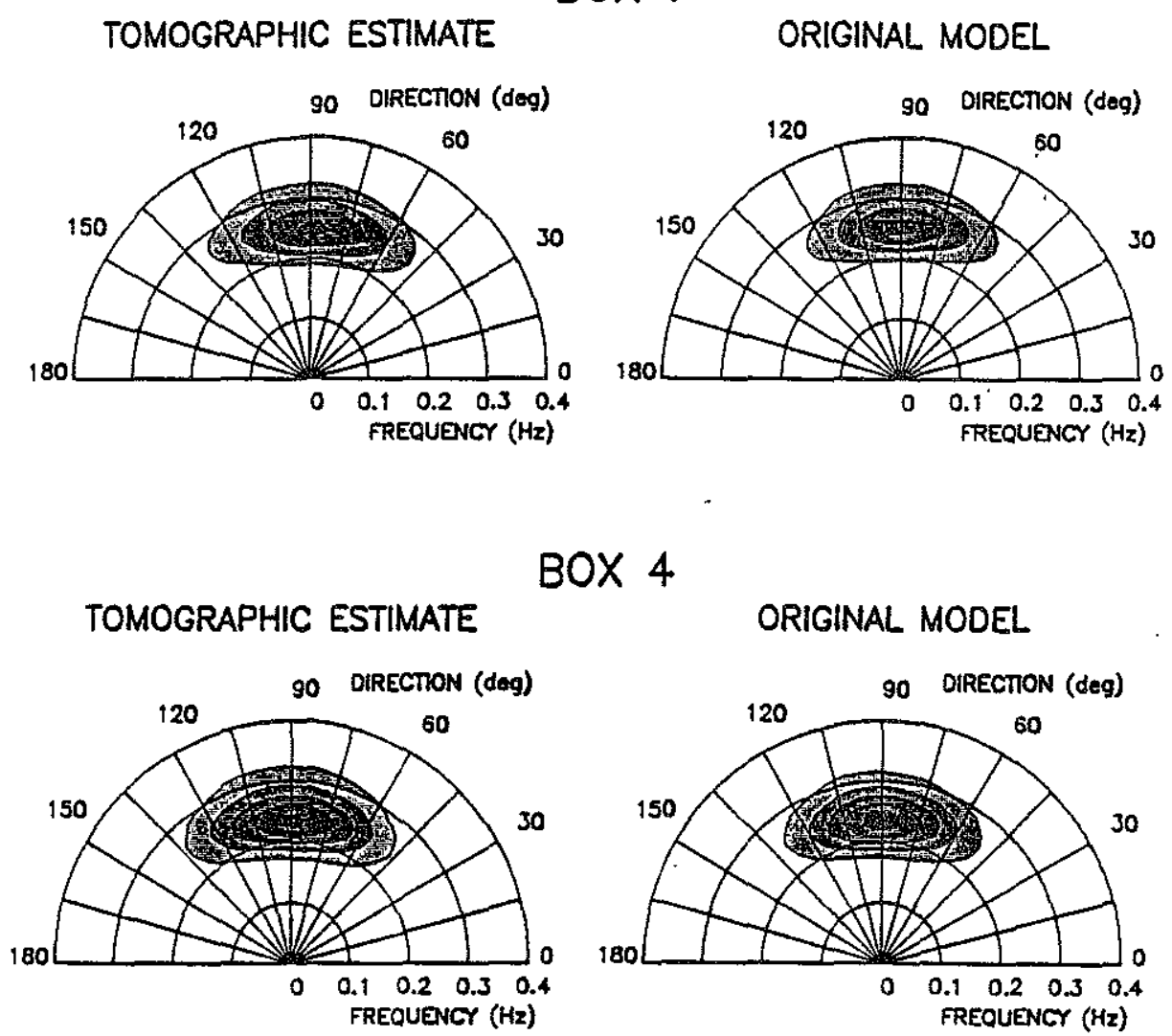

BOX 4
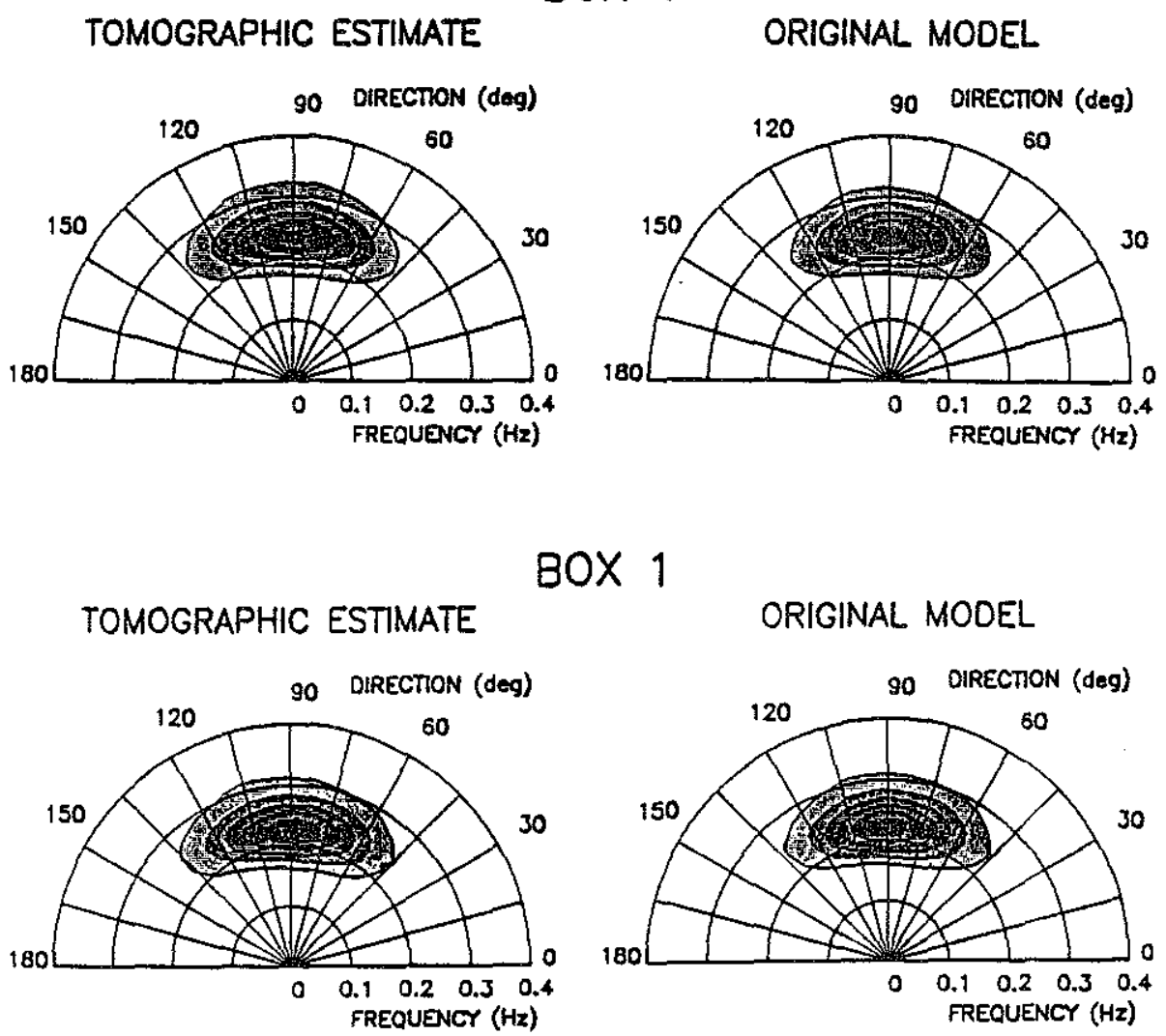

\section{BOX 1}

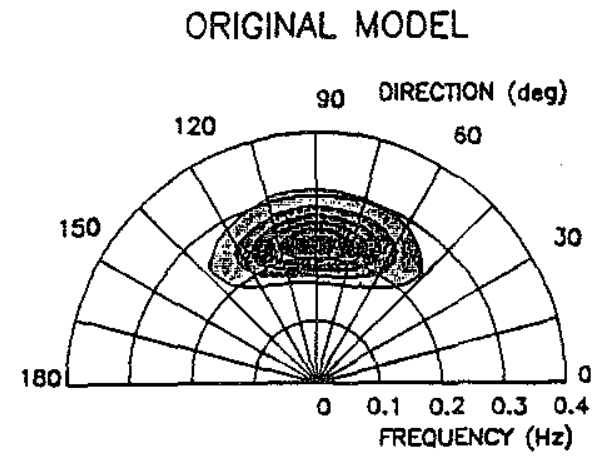

Figure 4.19: Contour plot of inversions of six rays (bounces 10-15) with noise for the frequency-directional spectra in boxes 1,4 , and 7 . See Fig. 4.7 for grey level key. 
BOX 8

TOMOGRAPHIC ESTIMATE
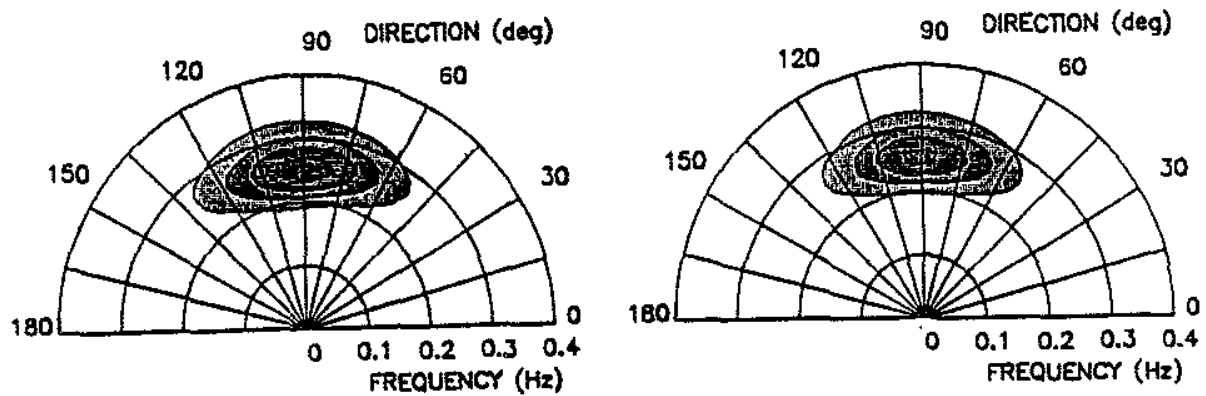

BOX 5

TOMOGRAPHIC ESTIMATE

90 DIRECTION (dog)
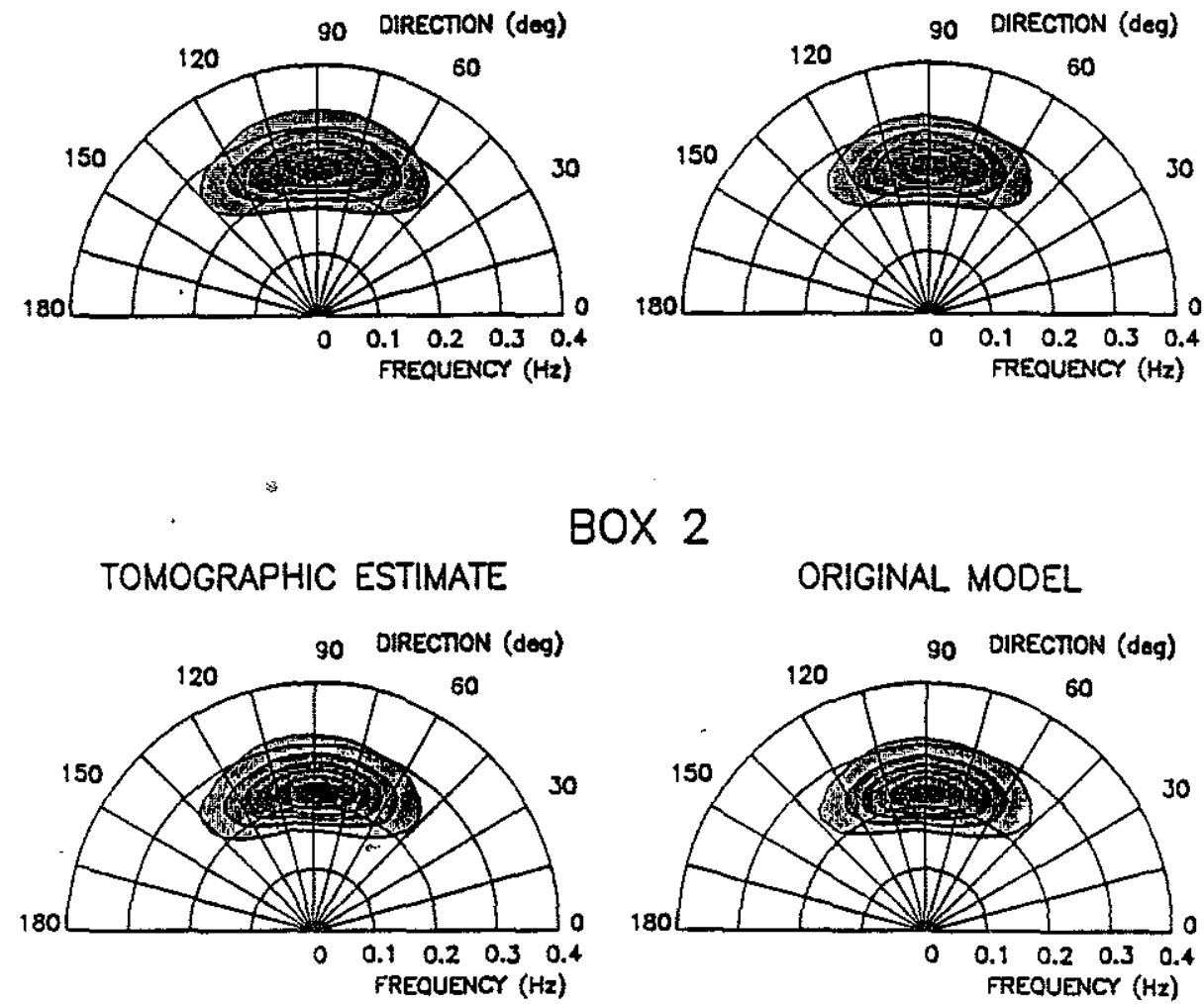

$\operatorname{BOX} 2$

ORIGINAL MODEL

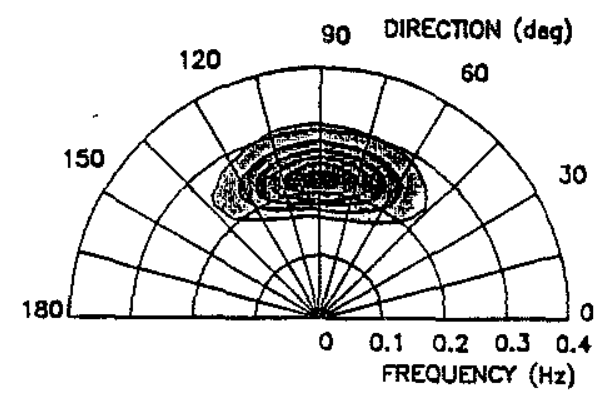

Figure 4.20: Contour plot of inversions of six rays (bounces 10-15) with noise for the frequency-directional spectra in boxes 2,5 , and 8 . See Fig. 4.7 for grey level key. 


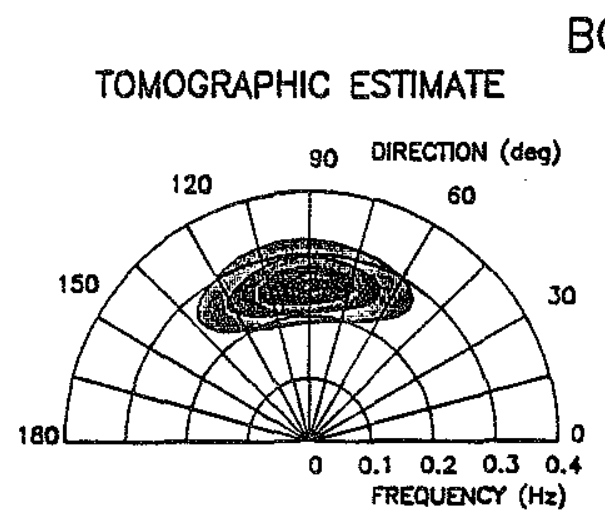

BOX 9
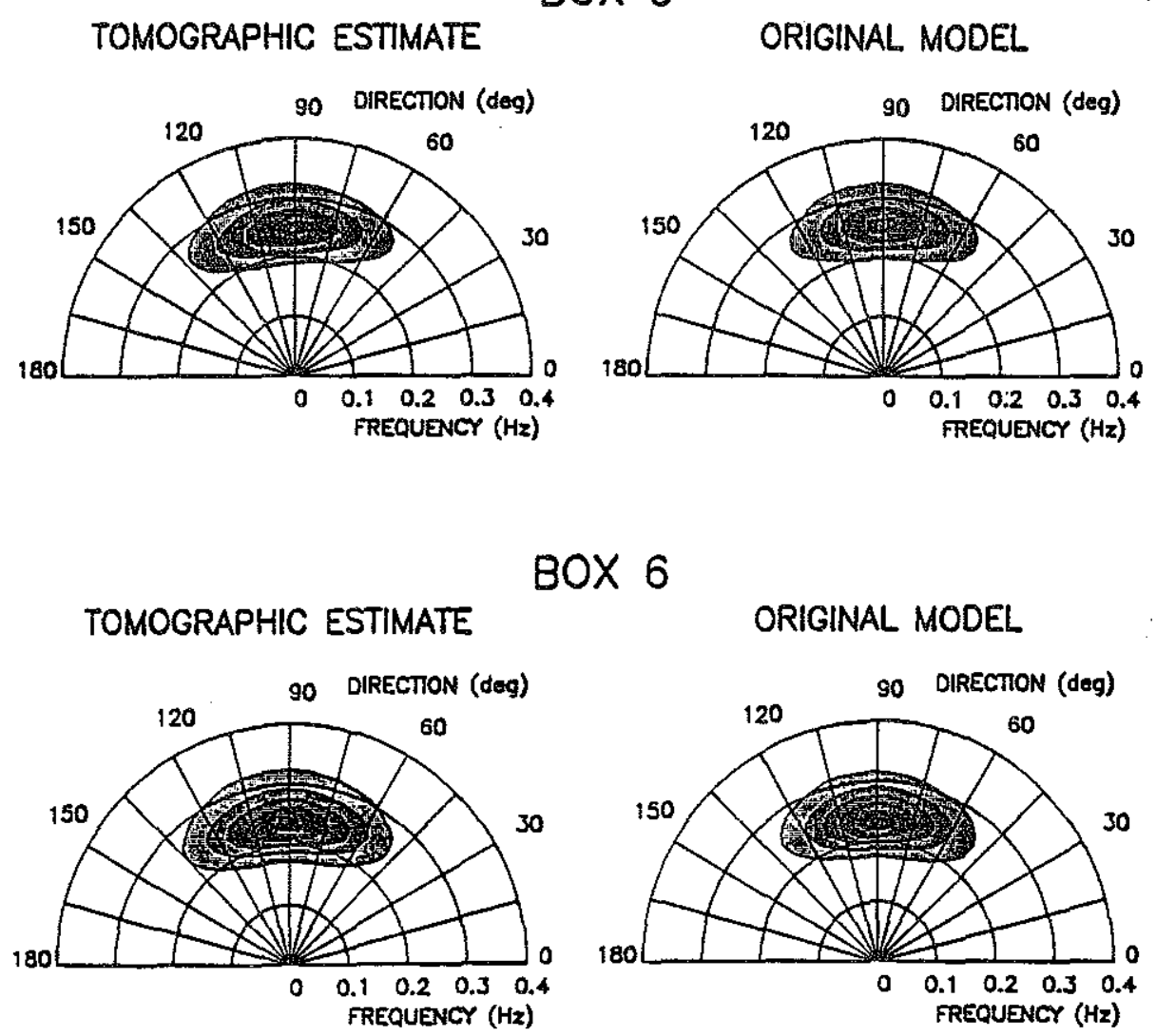

Box 6
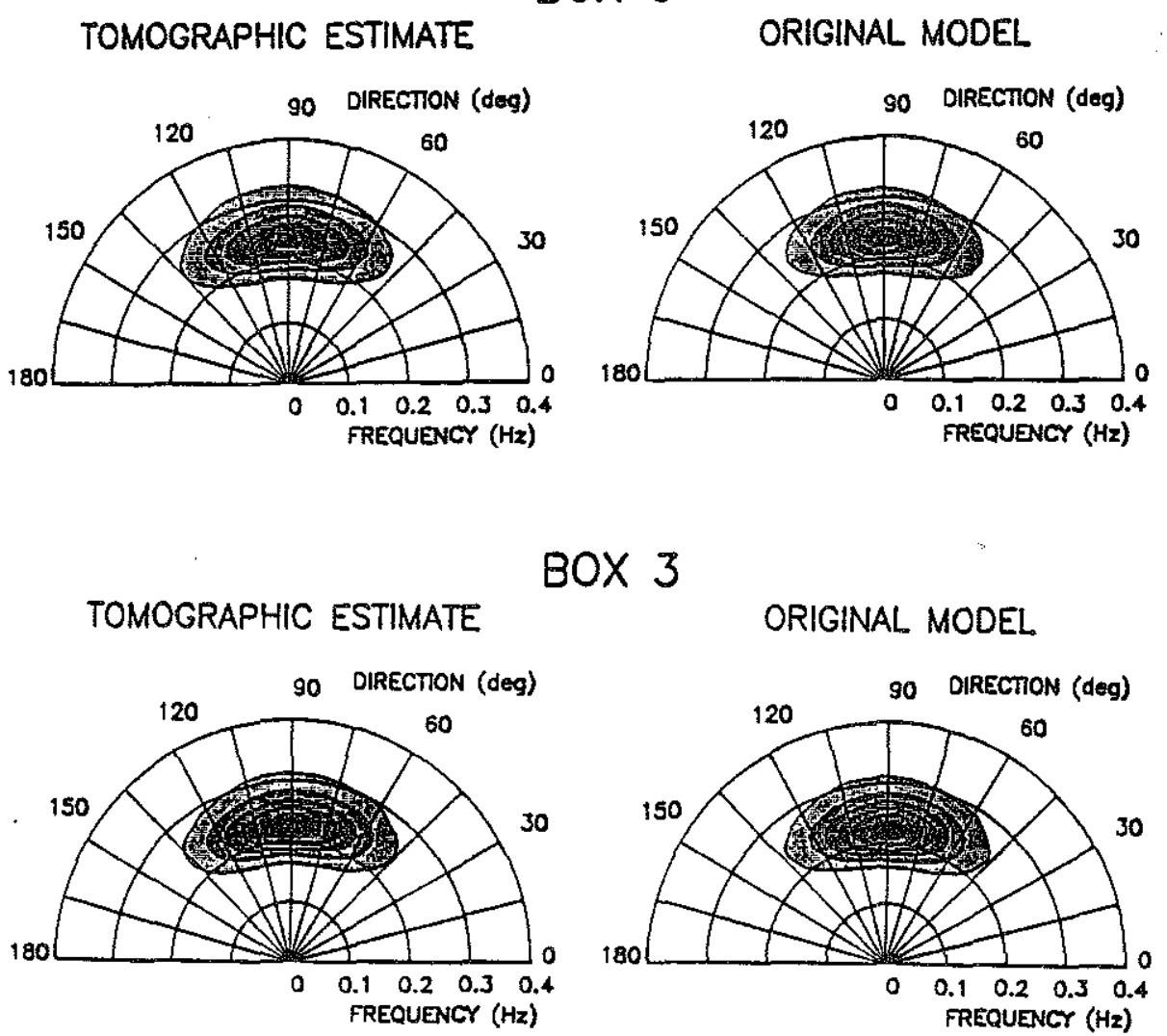

\section{BOX 3}

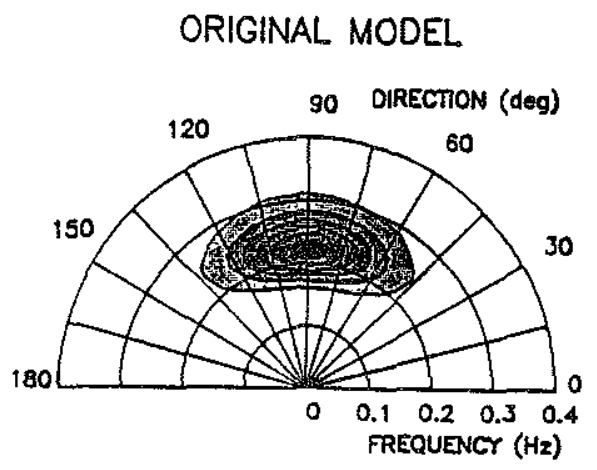

Figure 4.21: Contour plot of inversions of six rays (bounces 10-15) with noise for the frequency-directional spectra in boxes 3,6 , and 9. See Fig. 4.7 for grey level key. 

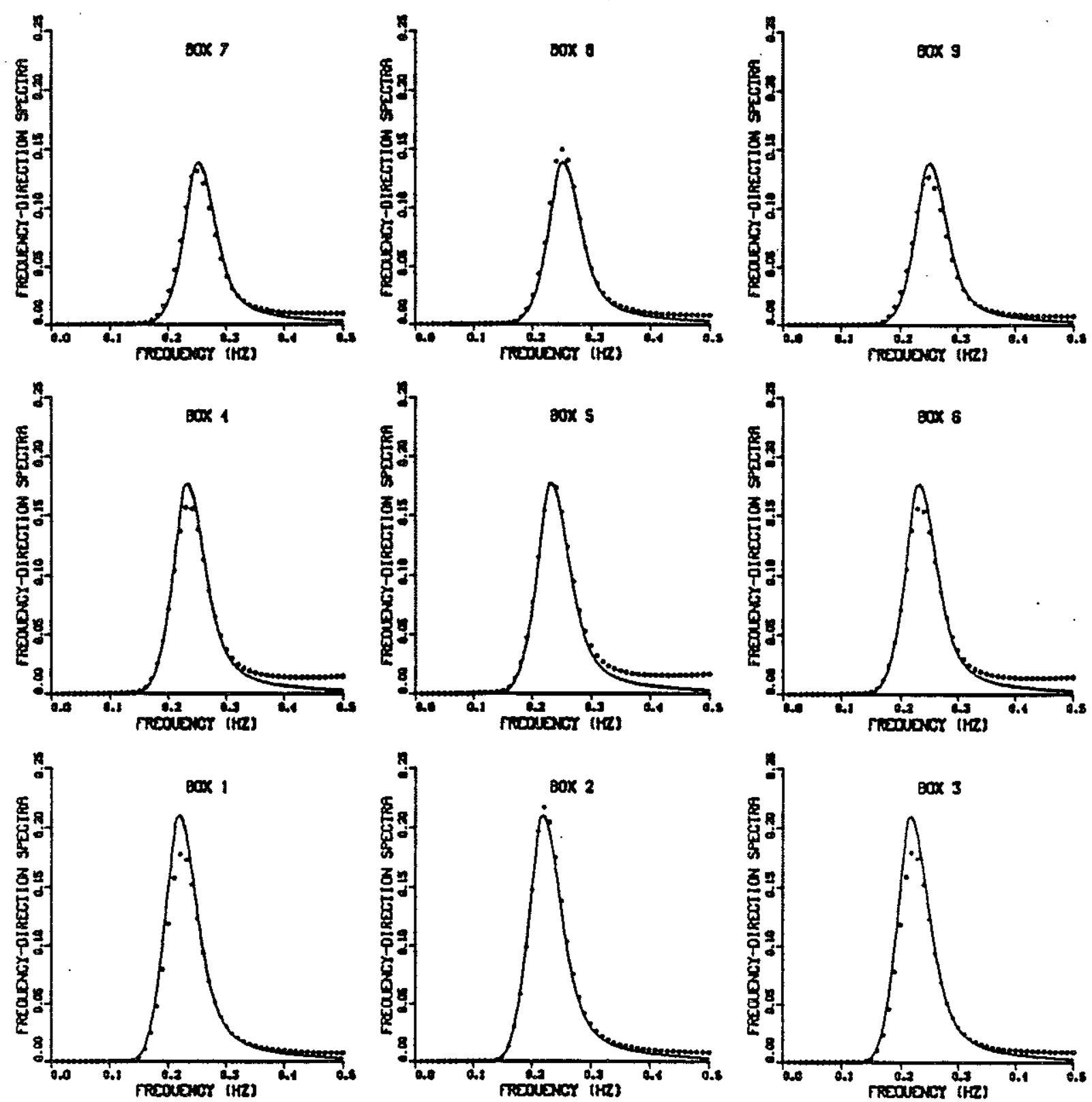

Figure 4.22: Inversion of six (bounces 10-15) rays with noise versus frequency at direction of 90 deg. Inverted data is given by a - while the original model is given by the smooth curve. 

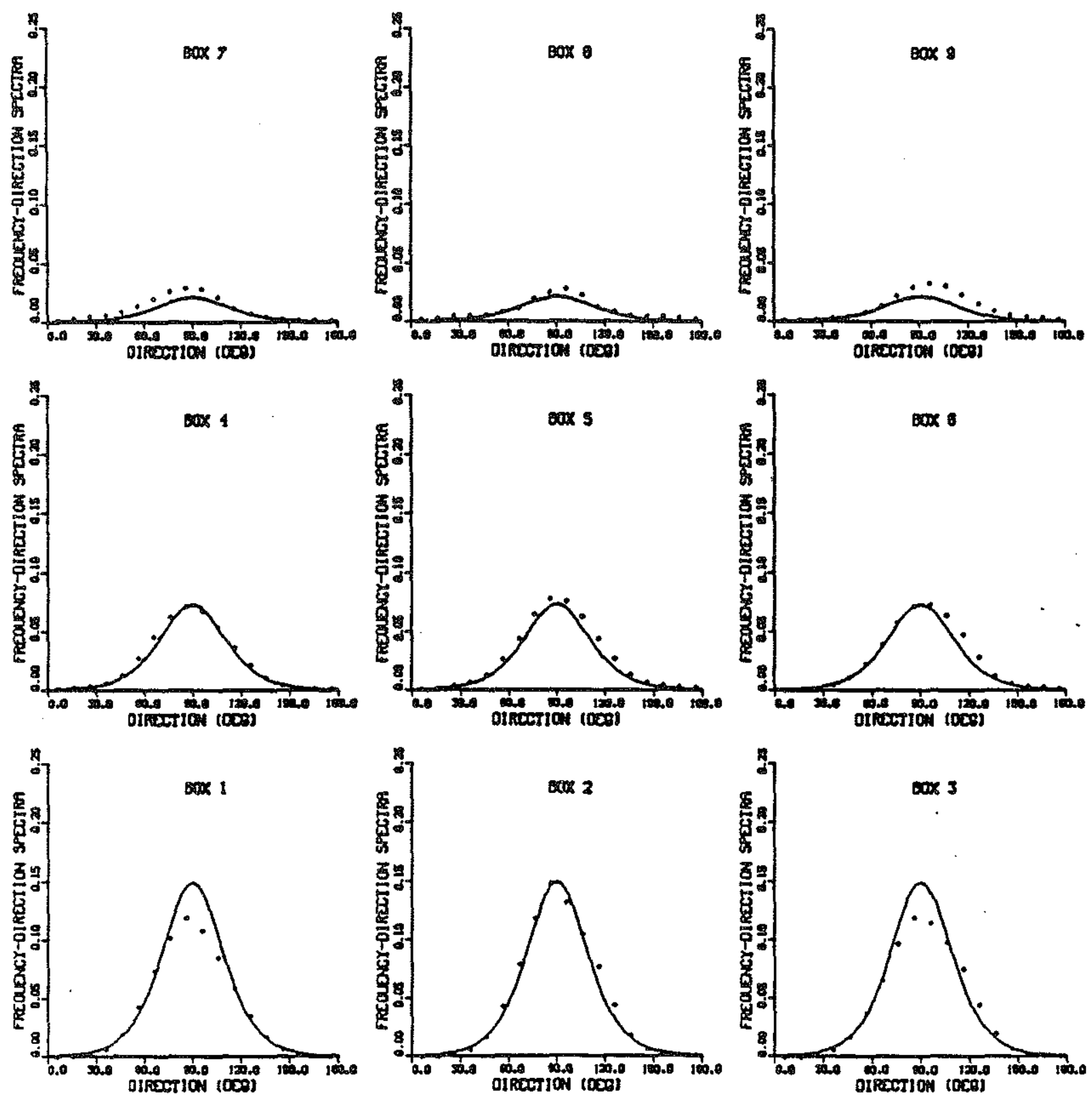

Figure 4.23: Inversion of six rays (bounces 10-15) with noise versus direction at a frequency of $0.2 \mathrm{~Hz}$. Inverted data is given by a - while the original model is given by the smooth curve. 


\subsubsection{Six Ray Inversion Results: Very Noisy Data}

The sensitivity of the inversions to more noisy data was assessed by using an acoustic noise level of $-91 \mathrm{~dB}$ instead of $-81 \mathrm{~dB}$. The results are shown in Figs. 4.24, 4.25 , and 4.26 in the contour plot format, in 4.27 in the plots versus frequency, and in 4.28 in the plots versus direction. Comparing these plots to their no noise and lesser noise counterparts, we can see dramatic differences at high frequencies. The directional dependence of the noise level in the contour plots is directly due to longer ranges the rays have to travel in some directions, thus losing SNR due to spreading, scattering losses, etc.

\subsubsection{Integration with Directional Wave Buoy}

Due to the generality of the inversion process, the integration of tomography with other oceanographic measuring systems such as satellite and temperature sensors for eddy-scale tomography is fairly straightforward $[6,71,5]$. We can also augment surface wave tomography with a more traditional wave measurement device such as a directional wave rider buoy. This is done as follows. The unknown frequency-direction vector $f$ given by Eq. (4.183) remains the same. We have to modify the other matrices as follows. The measurement vector $t$ now becomes

$$
t=\left[\begin{array}{c}
t^{\text {tomography }} \\
\cdots \\
t^{\text {wavebuoy }}
\end{array}\right]
$$

The matrix $\mathrm{W}$ must also be augmented to normalize the errors between the two sensor systems. For an example, we assume that a directional wave buoy is located at coordinates $(10 \mathrm{~km}, 10 \mathrm{~km})$ in the map given in Fig. 4.4. The kernel matrix must be augmented to include a measurement of $f$ in box 1 . Note that the inversion is now frequency-dependent however, and so must be performed at every frequency 

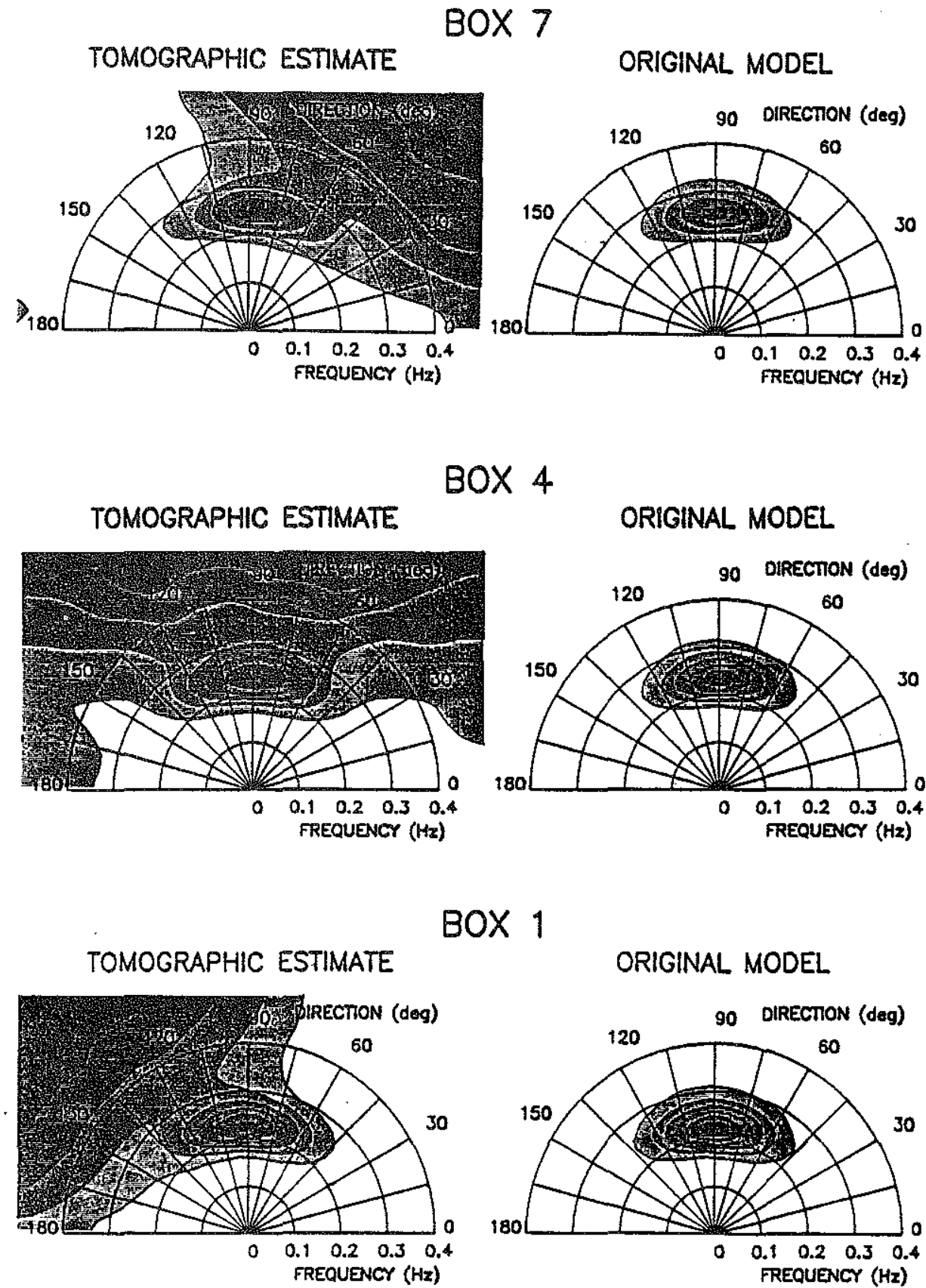

Figure 4.24: Contour plot of inversions of six rays (bounces 10-15) with $10 \mathrm{~dB}$ more noise for the frequency-directional spectra in boxes 1 , 4 , and 7 . See Fig. 4.7 for grey level key. 

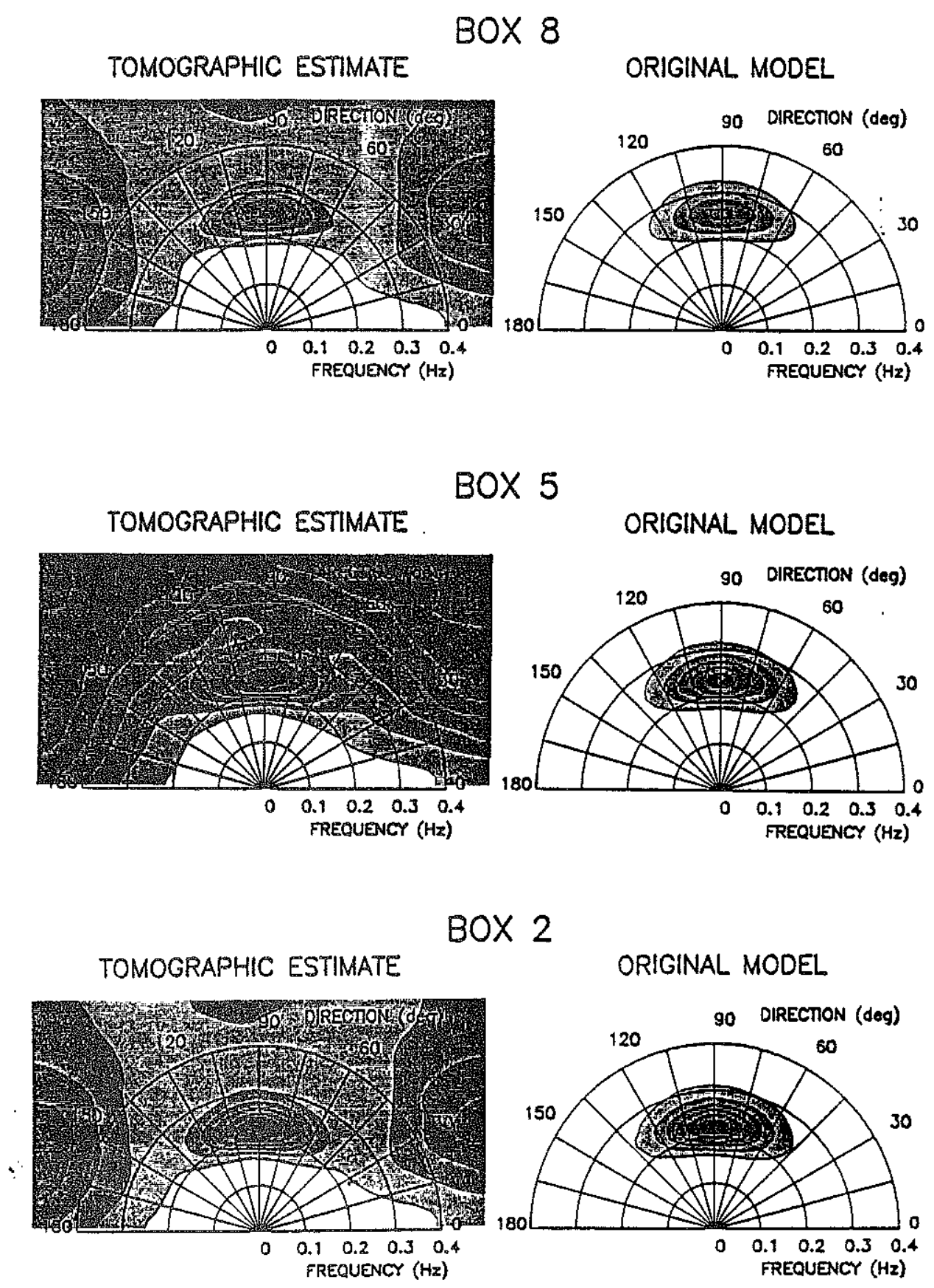

Figure 4.25: Contour plot of inversions of six rays (bounces 10-15) with $10 \mathrm{~dB}$ more noise for the frequency-directional spectra in boxes 2,5 , and 8 . See Fig. 4.7 for grey level key. 
$30 \times 9$

TOMOGRAPHIC ESTIMATE

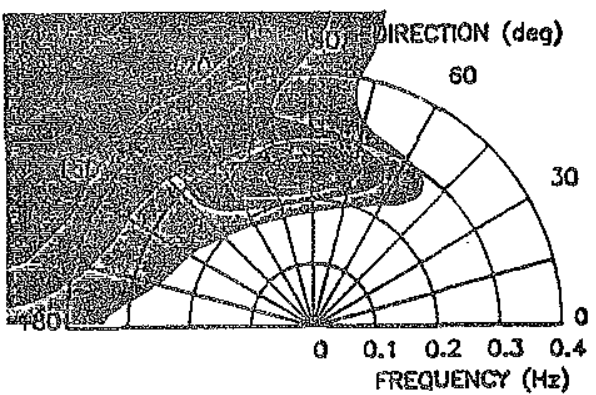

ORIGINAL MODEL

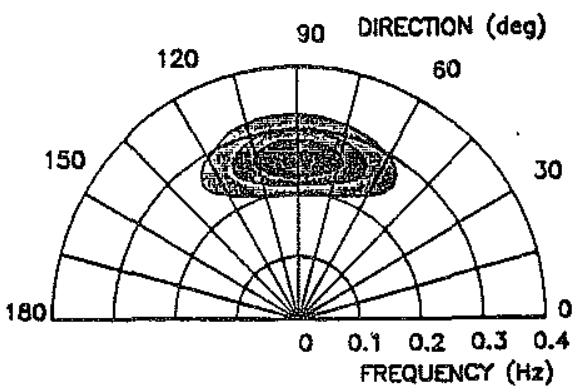

BOX 6

TOMOGRAPHIC ESTMATE

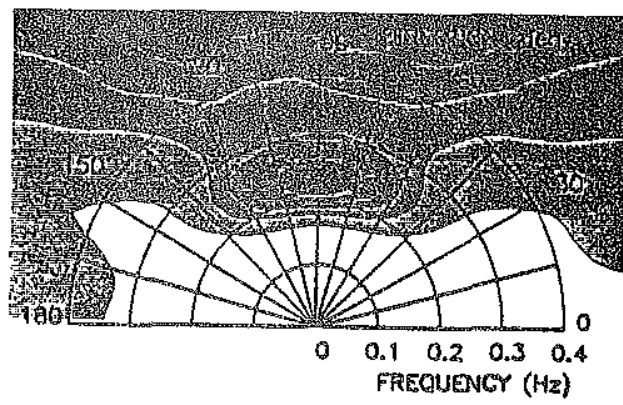

ORIGINAL MODEL
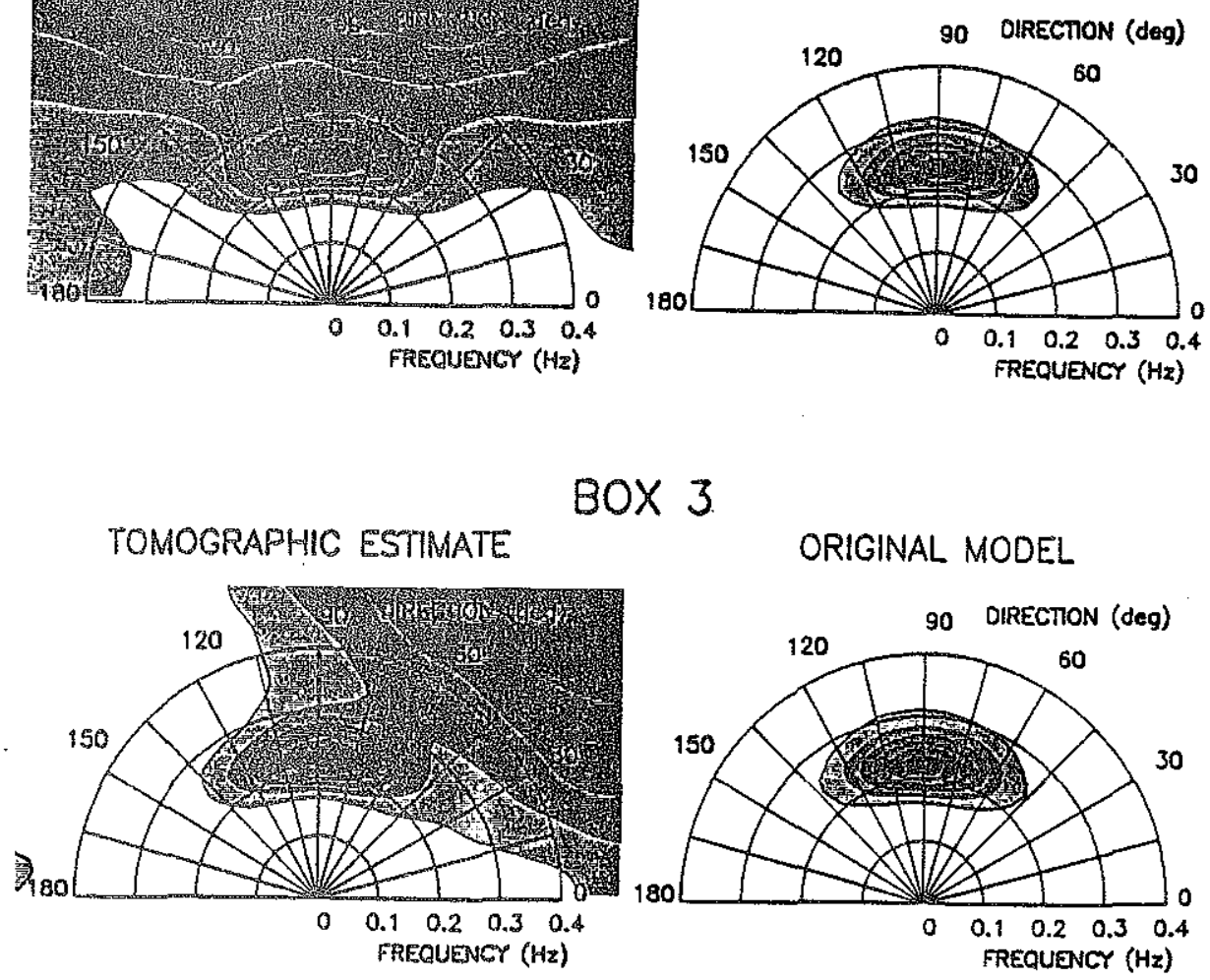

Figure 4.26: Contous plot of inversions of six (bounces 10-15) rays with $10 \mathrm{~dB}$ more noise for the frequency-directional spectra in boxes 3, 6, and 9. See Fig. 4.7 for grey level key. 

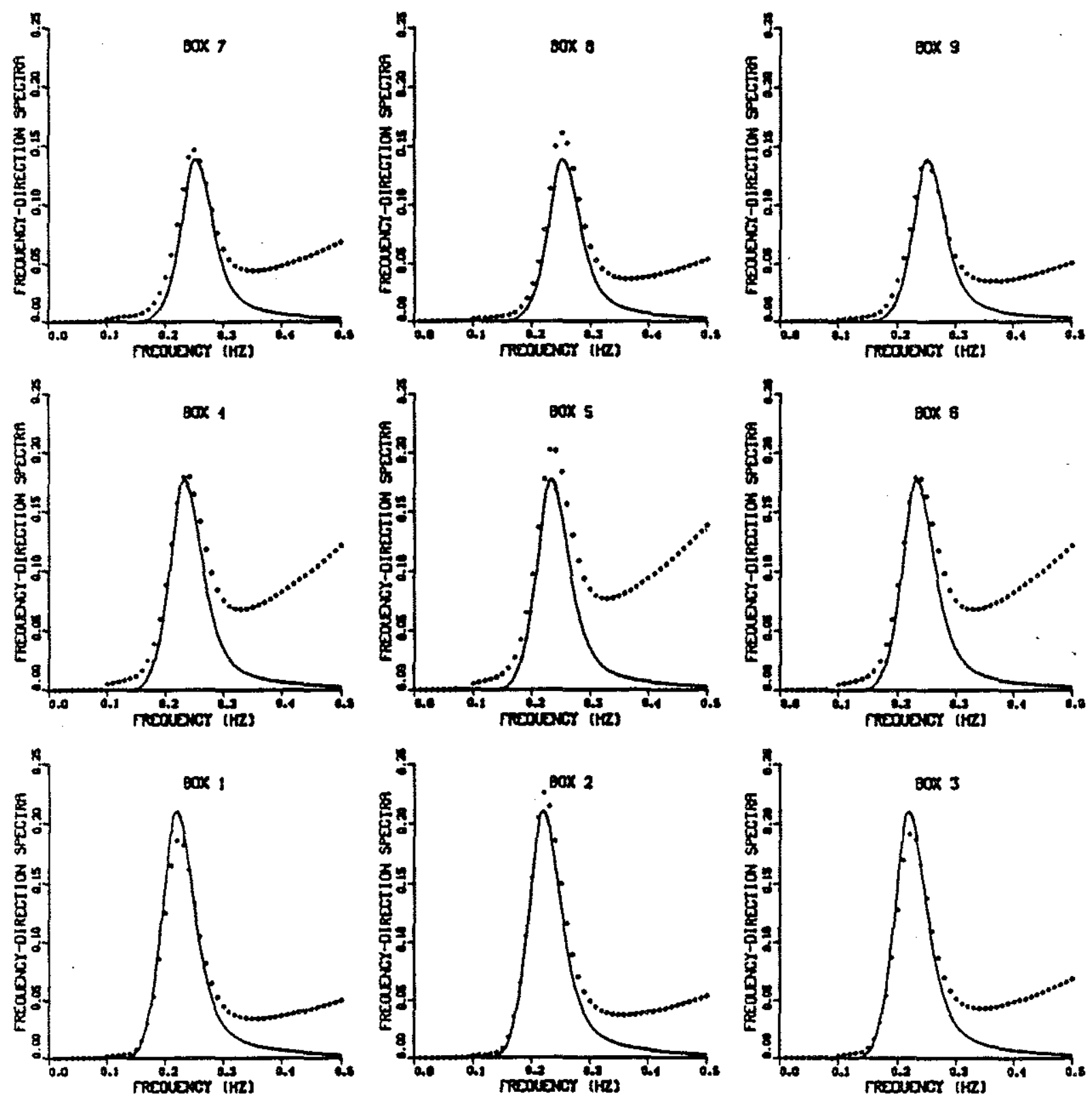

Figure 4.27: Inversion of six rays (bounces 10-15) with $10 \mathrm{~dB}$ more noise versus frequency at direction of $90 \mathrm{deg}$. Inverted data is given by a - while the original model is given by the smooth curve. 

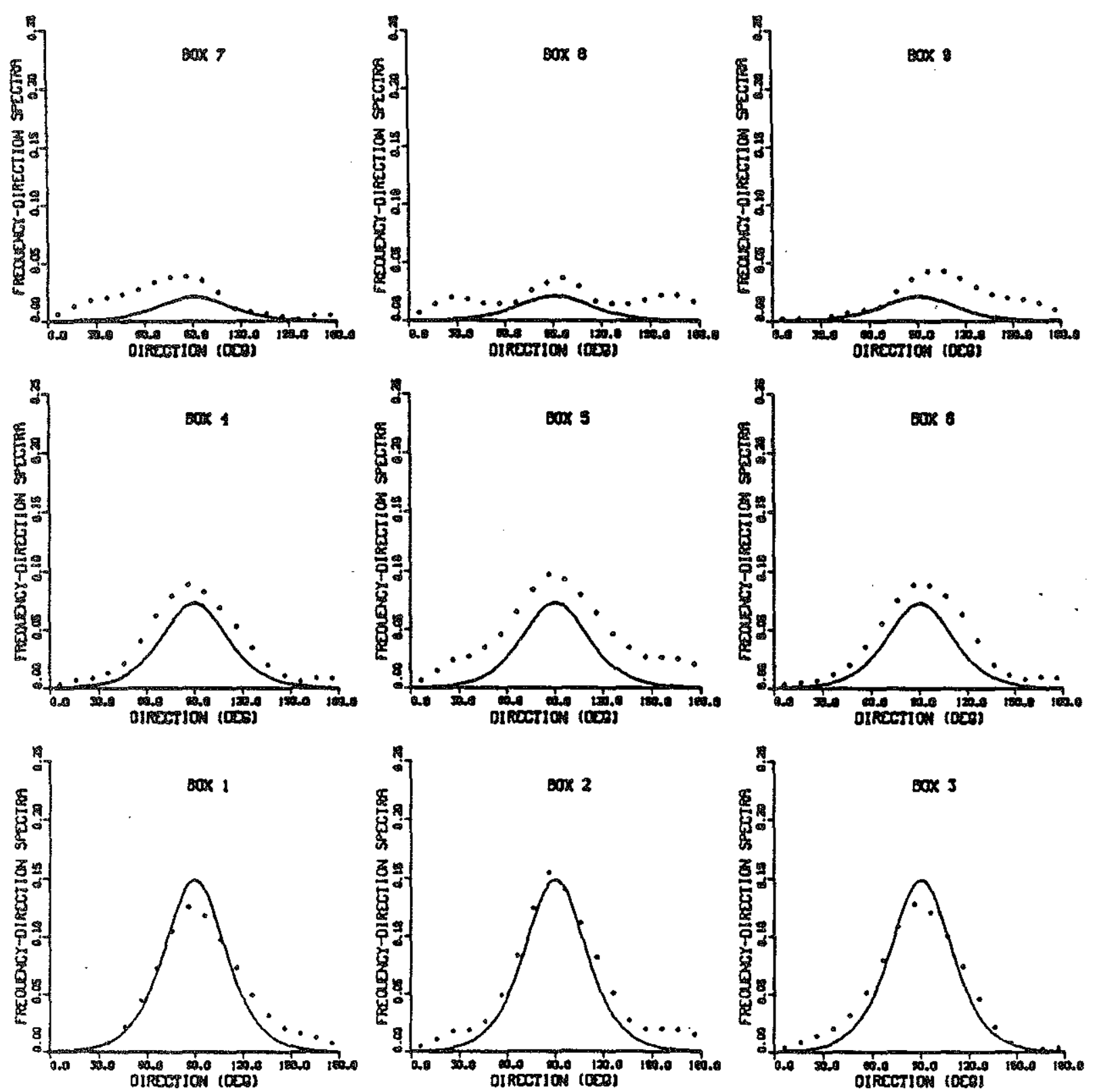

Figure 4.28: Inversion of six rays (bounces 10-15) with $10 \mathrm{~dB}$ more noise versus direction at a frequency of $0.2 \mathrm{~Hz}$. Inverted data is given by a while the original model is given by the smooth curve. 
desired. This may multiply computations by a factor of 25 or so, a large but not impossible increase.

Figures 4.29 and 4.30 show the results of an integrated measurement using modes and the directional wave buoy. All measurements were noise free. As expected, in box 1 , where the buoy is located, the inversion results are improved over those in Figs. 4.11 and 4.12. The results in box 1 are just about perfect but the results in the adjoining boxes are also improved. The least improved box is number 9 diagonally opposite box 1 with the buoy. The correlation between these boxes is the least and the results reflect that fact.

\subsubsection{Three Ray Inversion: Noisy Data}

For comparison, we also performed an inversion on three rays with 13 to 15 surface bounces. The rays were the same used in Section 4.2.3, with error introduced by the typical SNR used in tomography experiments. The results are shown in Figs. 4.31 and 4.32. As compared with the six ray inversions in Figs. 4.22 and 4.23, the three ray inversion results are poorer in estimating the spectrum near the peak frequencies. The poor results are due to the less dense sampling of the sea surface by the three rays as compared to the six rays.

\subsubsection{Nine Ray Inversion: Noisy Data}

We also performed an inversion on nine rays with 1 to 9 surface bounces, with error introduced by the same SNR as described in in Section 4.2.3. The results are shown in Figs. 4.33 and 4.34. As compared with the six ray inversions in Figs. 4.22 and 4.23 , the nine ray inversion results are poorer in estimating the spectrum near the peak frequencies. But also note the performance at higher frequencies in Fig. 4.33. The noise effect is more prominent, even though we are using more 

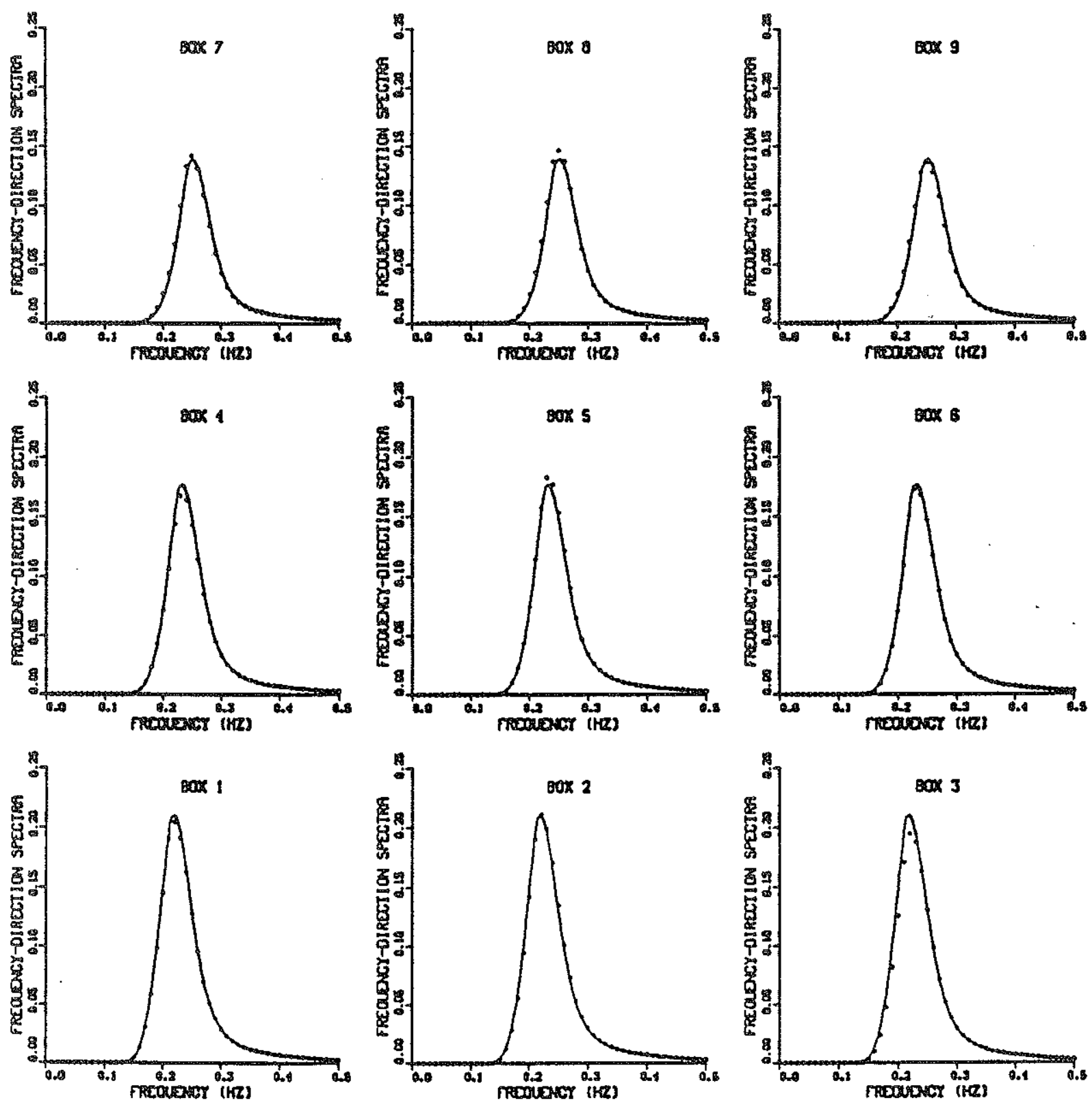

Figure 4.29: Inversion of six rays (bounces 10-15) and directional wave buoy versus frequency at direction of 90 deg. Inverted data is given by $a$ - while the original model is given by the smooth curve. 

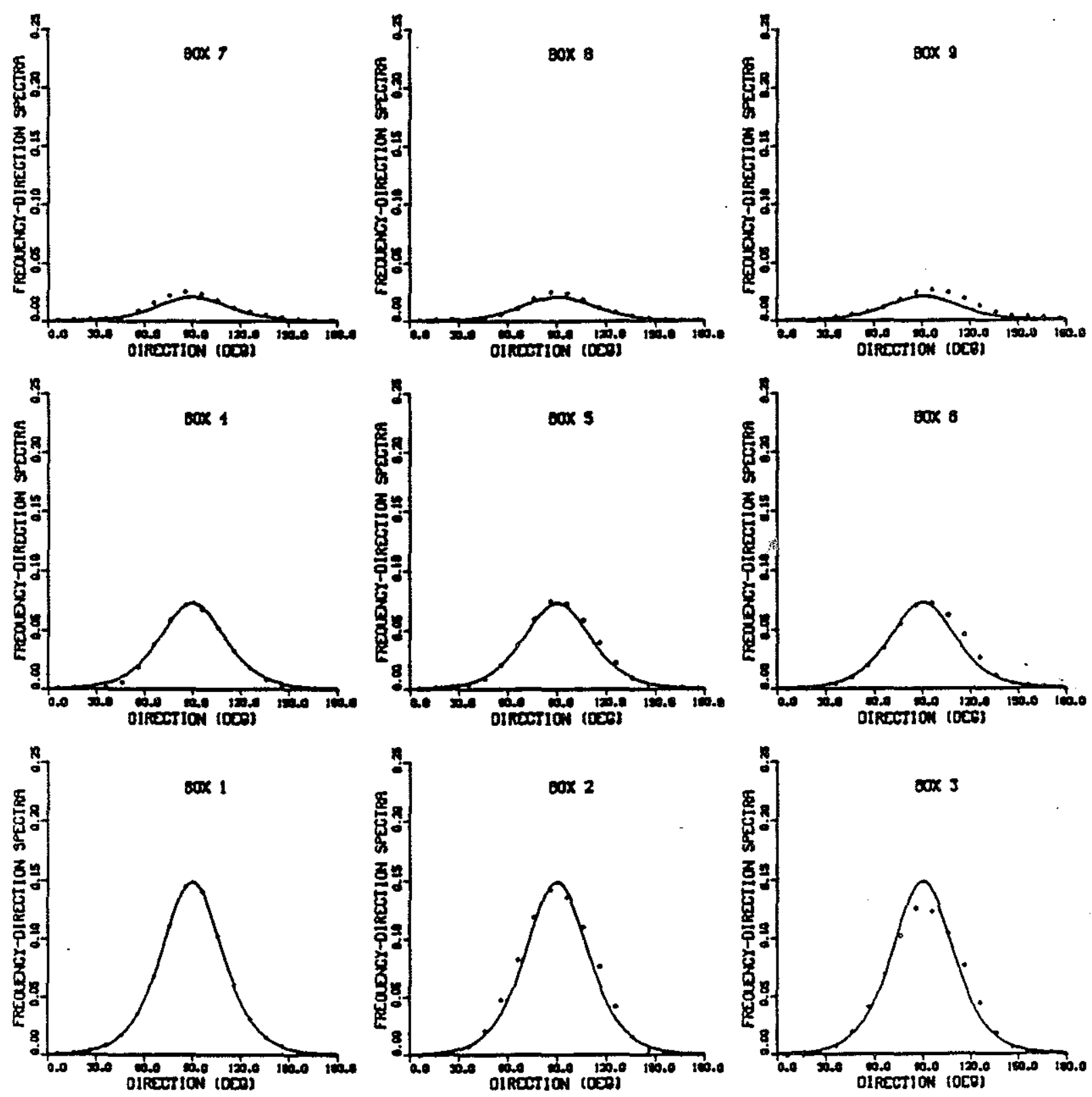

Figure 4.30: Inversion of six rays (bounces 10-15) and directional wave buoy versus direction at a frequency of $0.2 \mathrm{~Hz}$. Inverted data is given by a $\bullet$ while the original model is given by the smooth curve. 

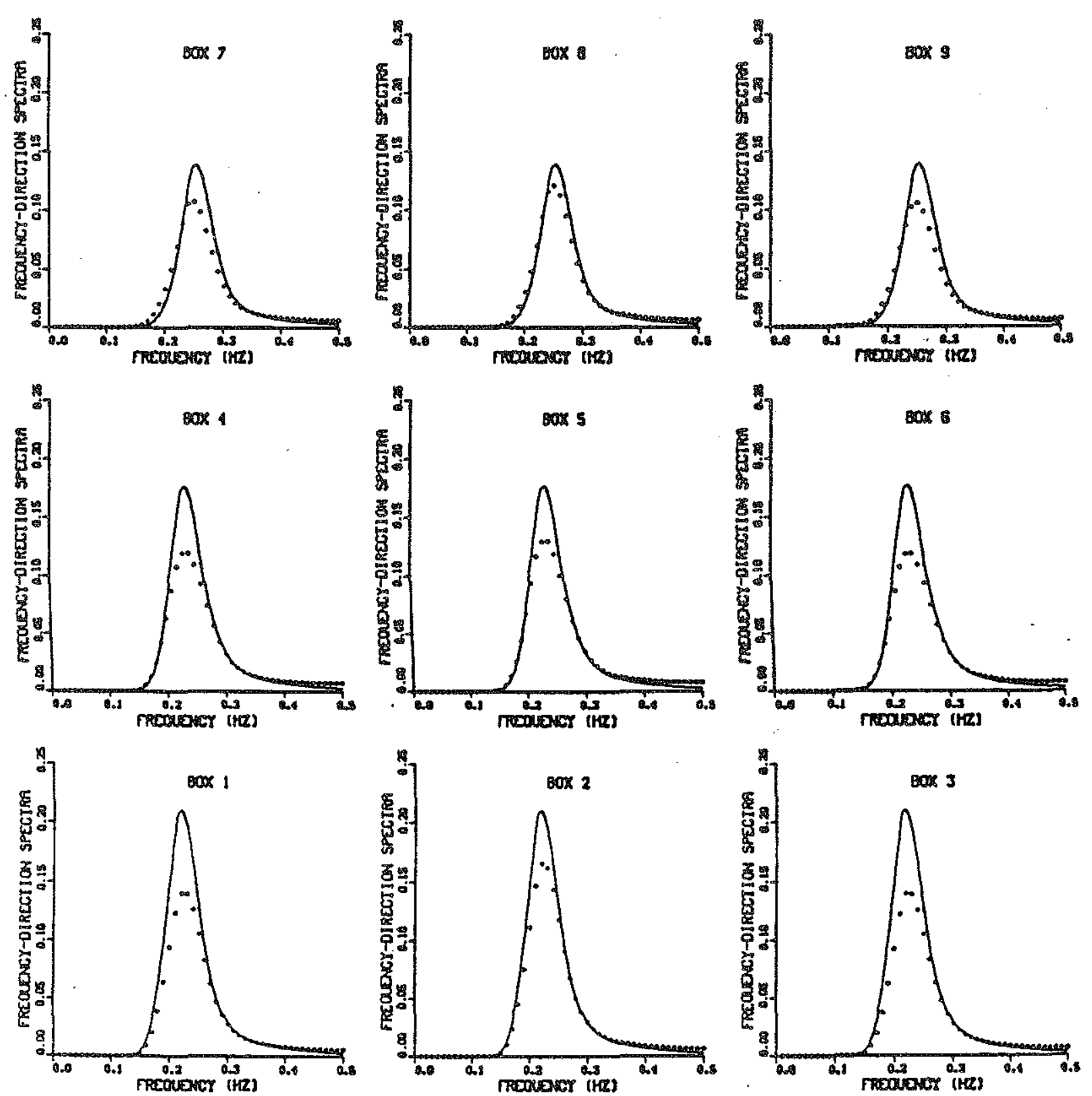

Figure 4.31: Inversion of three rays (bounces 13-15) with noise versus frequency at direction of $90 \mathrm{deg}$. Inverted data is given by $a \cdot$ while the original model is given by the smooth curve. 

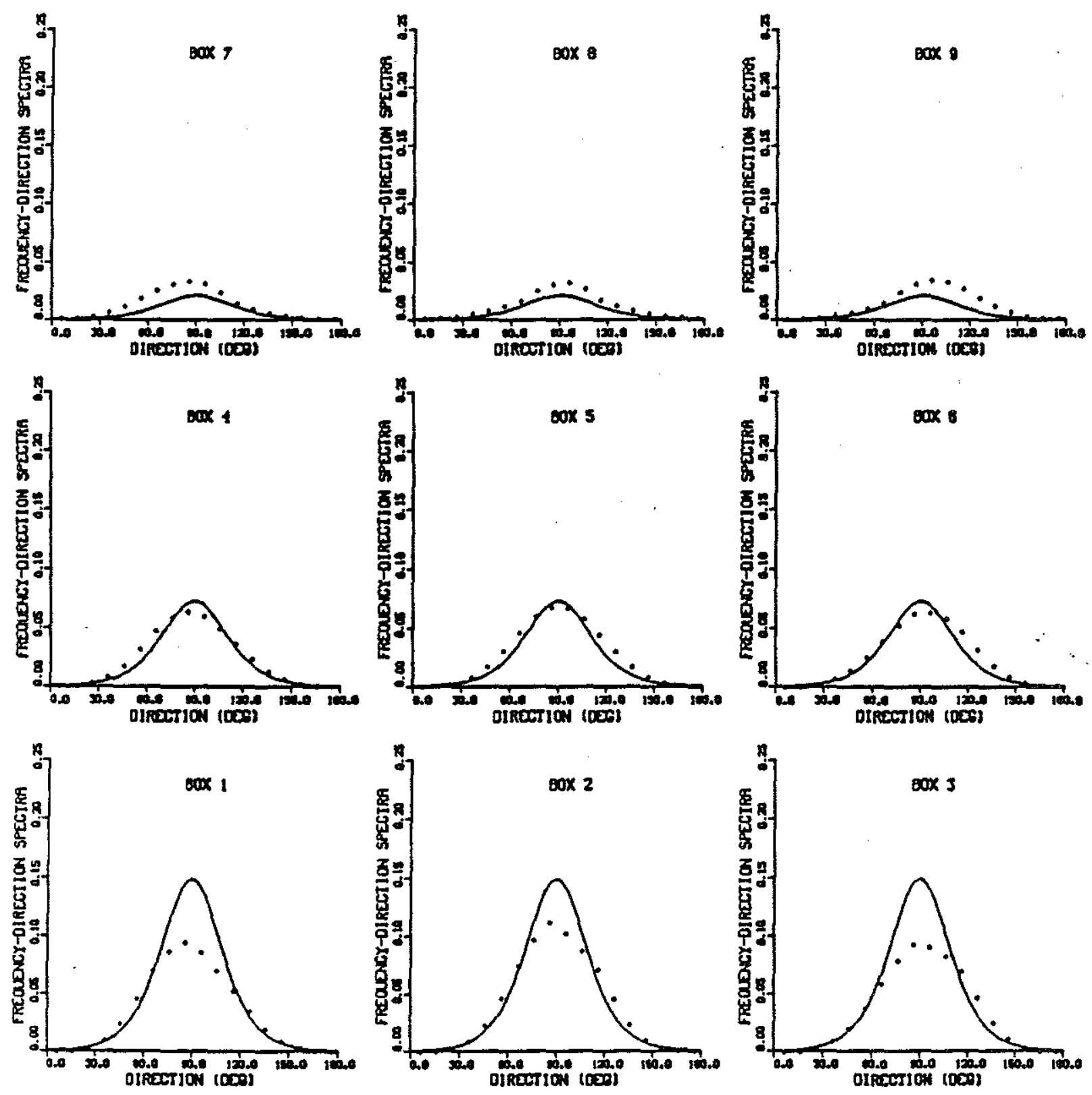

Figure 4.32: Inversion of three rays (bounces 13-15) with noise versus direction at a frequency of $0.2 \mathrm{~Hz}$. Inverted data is given by a $\bullet$ while the original model is given by the smooth curve. 
rays than in Section 4.2.3. The cause of this effect is the fact that the rays with bounces between 1 and 9 have lower rms travel time perturbation because of the fewer bounces. With the same spectral noise level and lower signal level, the noise is amplified as compared to the case where we use rays with bounces between 10 and 15.

\subsection{Resolution and Variance}

We have plotted the variance as a function of box and direction in Fig. 4.35 for the matrices used in the 6 ray inversions. Note the large variances at directions above 90 deg in boxes 1 and 9. The errors depicted in Figs. 4.24 and 4.26 are large where the variance is large in Fig. 4.35, as we would expect. The lack of angular dependence of the variance in box 5 in Fig. 4.35 can be easily seen in Fig. 4.25.

In Section 4.2.5, we added a directional wave buoy in box 1 that measured the frequency-direction spectrum with no noise. The variance for that integrated measurement is shown in Fig. 4.36. We see that, in box 1, the variance has gone to zero. In the adjacent boxes, the variance is lowered compared to the acoustics-only case of Fig. 4.35. In the boxes furthest away from box 1, the improvement in the solution variance is the least as we would have expected.

Figure 4.37 shows the resolution that we can obtain in box 2 at 90 deg and leakage into the other boxes. Because of the smoothing introduced to enable the inversion to work, we tend to smooth through boxes 1 and 3 to the extent shown in the plots. We do better across the other boxes that are perpendicular to the shore. This is because the correlations between boxes parallel to the shore (e.g. boxes 1 , 2, and 3) are higher than those perpendicular to the shore (e.g. boxes 1, 4, and 7). Figure 4.38 show a similiar plot for the normalized resolution we obtain in box 5 at $90 \mathrm{deg}$. The high smoothing between boxes 4,5 , and 6 is evident in the high side 

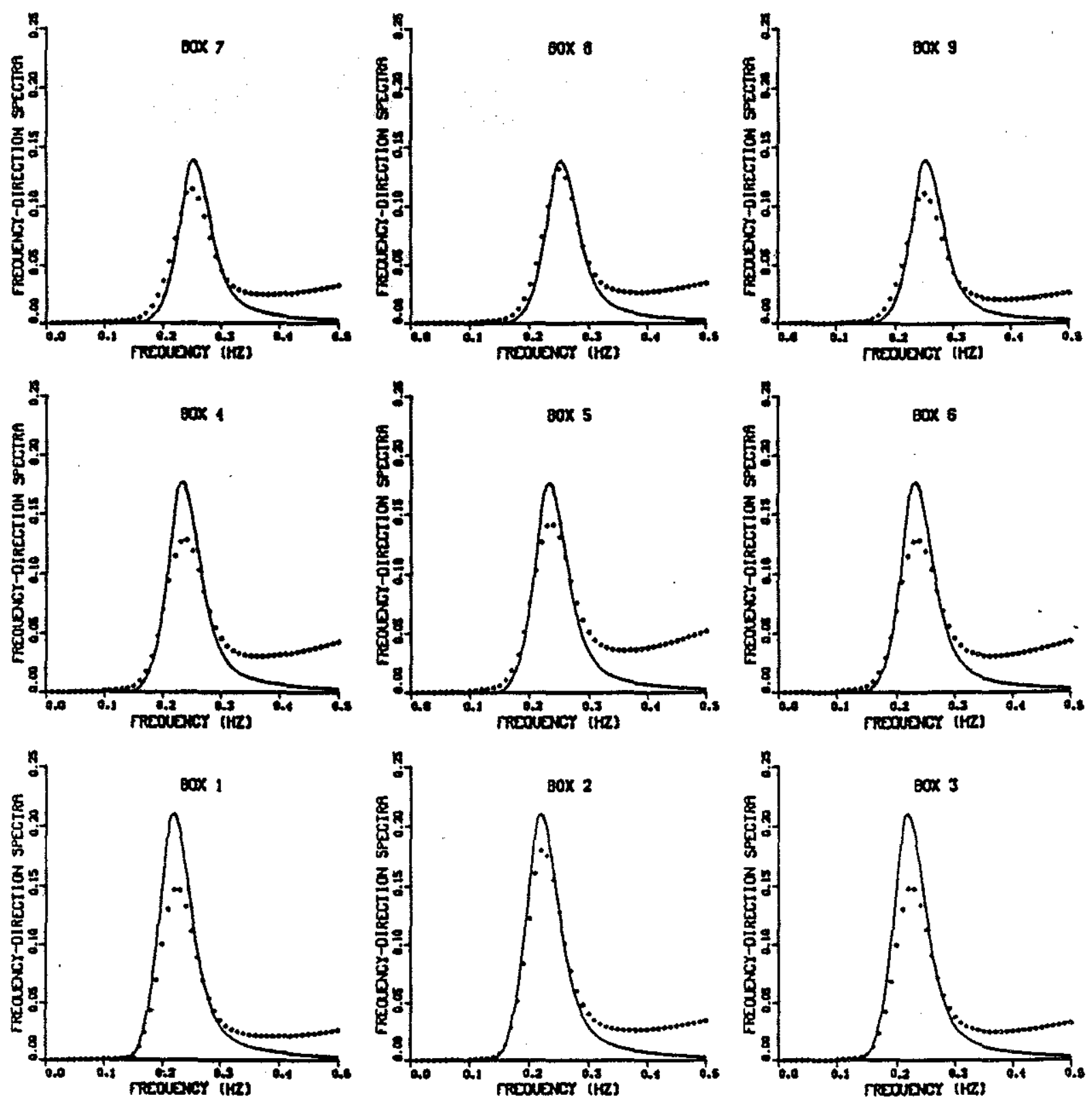

Figure 4.33: Inversion of nine rays (bounces 1-9) with noise versus frequency at direction of $90 \mathrm{deg}$. Inverted data is given by $a$ - while the original model is given by the smooth curve. 

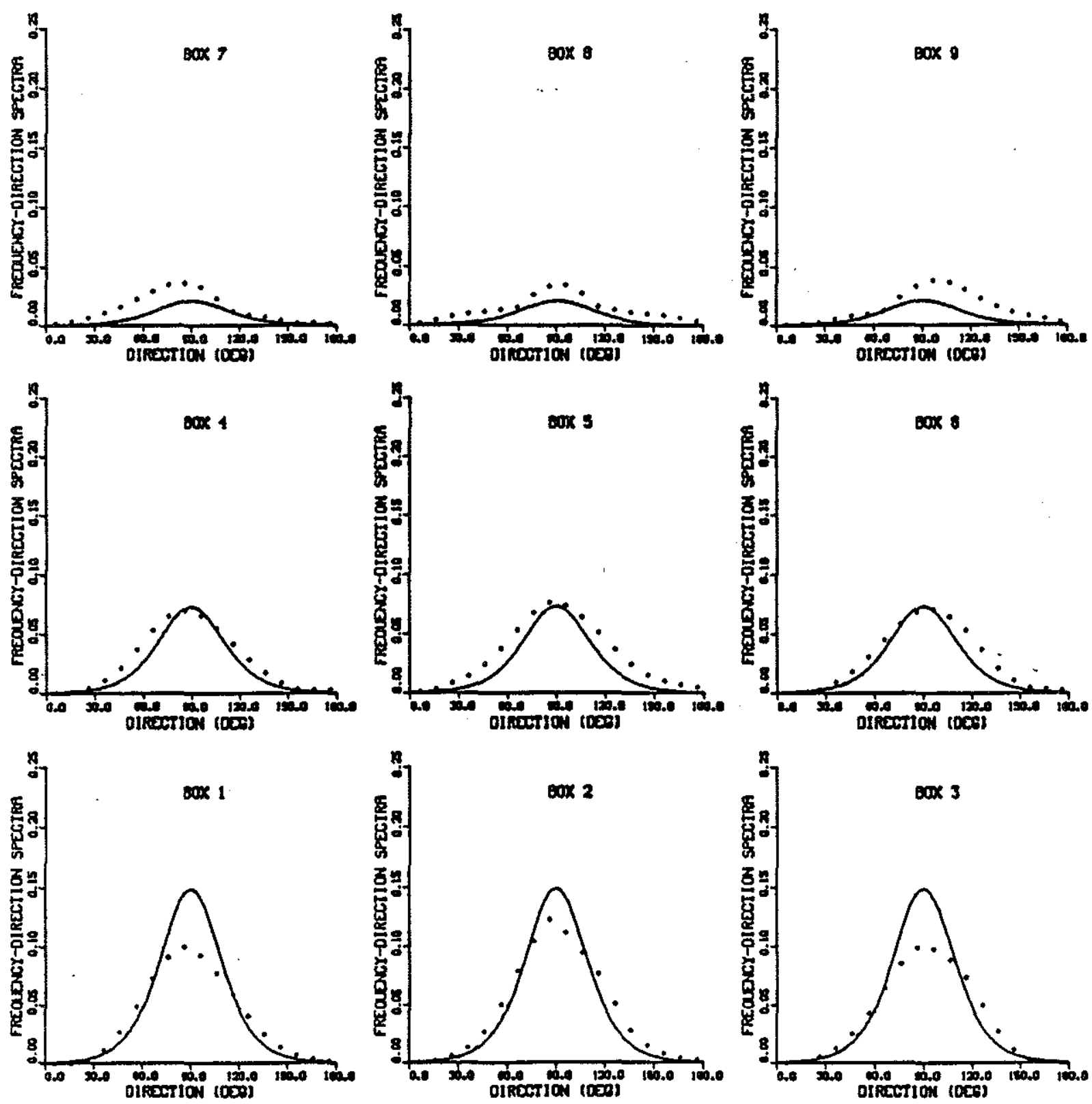

Figure 4.34: Inversion of nine rays (bounces 1-9) with noise versus direction at a frequency of $0.2 \mathrm{~Hz}$. Inverted data is given by a $\bullet$ while the original model is given by the smooth curve. 

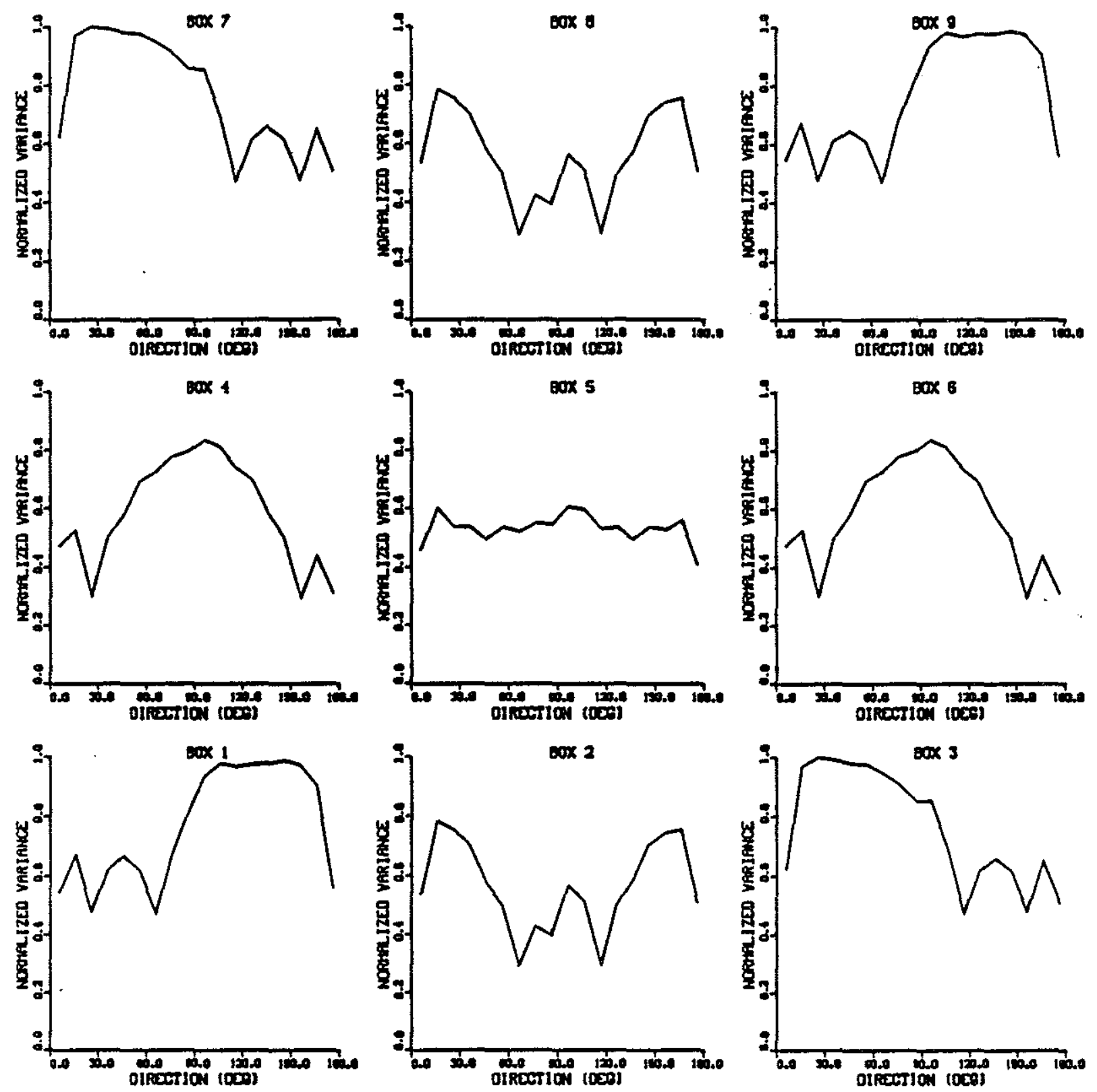

Figure 4.35: Variance of the ray inversion solution. 

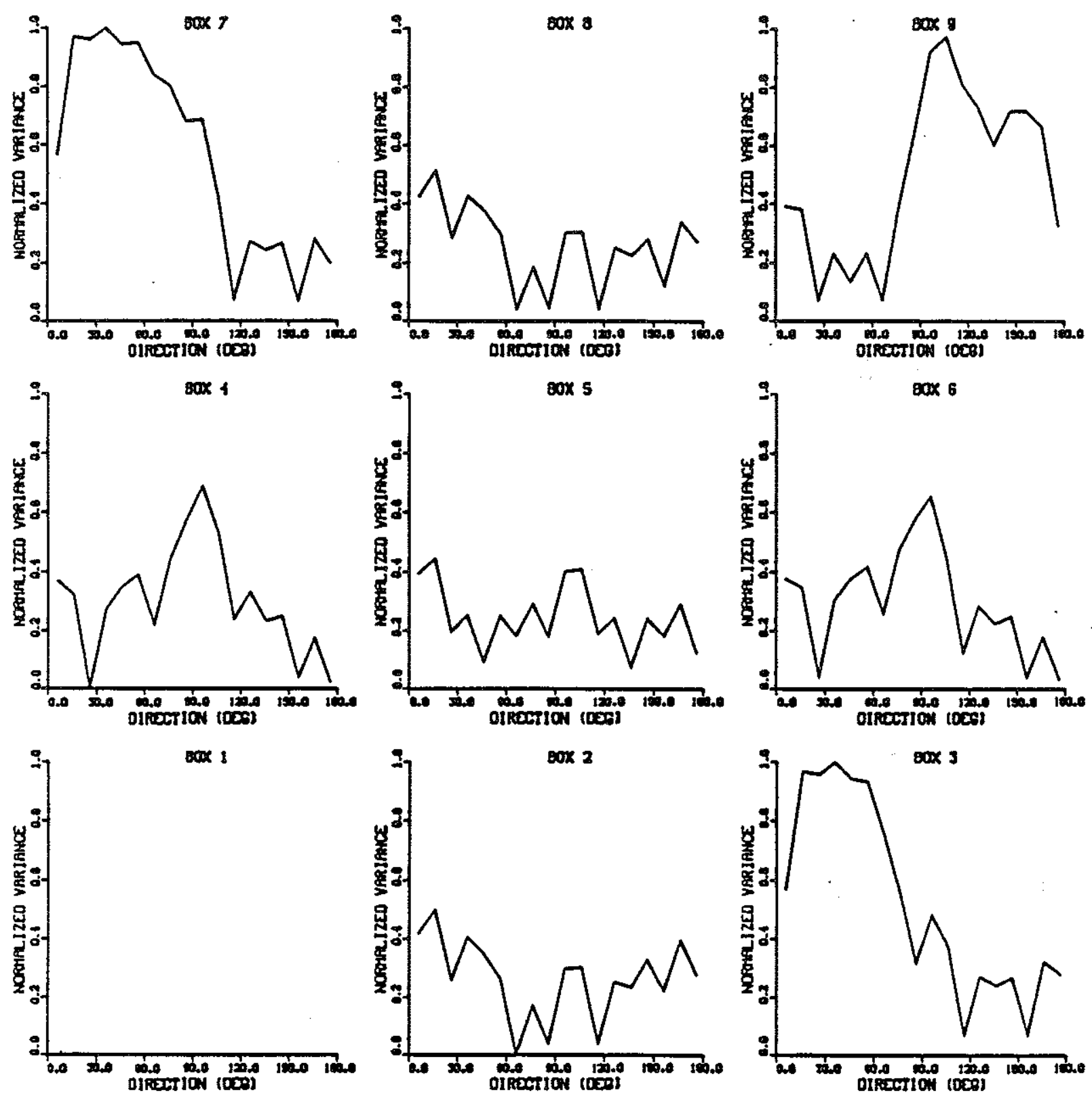

Figure 4.36: Variance of the ray inversion solution using a surface wave directional buoy in box 1 . 
lobes in boxes 4 and 6. Our inversion (with the current correlation structure) will not admit solutions that change drastically across the shore-parallel boxes.

In direction, we smoothed with a method similiar to a cubic spline. We did this because we had no data on the spectrum in between the directions perpendicular to the acoustic mode path as we illustrated in Fig. 4.1. The cost we pay for this, as Figs. 4.37 and 4.38 show, by not being able to resolve features in the spectrum that are less than 20 deg wide. Thus, any compact swell spectrum could slip in between the sensing directions and we would miss it all together. We could have predicted this without a calculation by noting that the acoustic paths are 22.5 deg apart, and spectral features less than this might slip between the sensitive directions. 

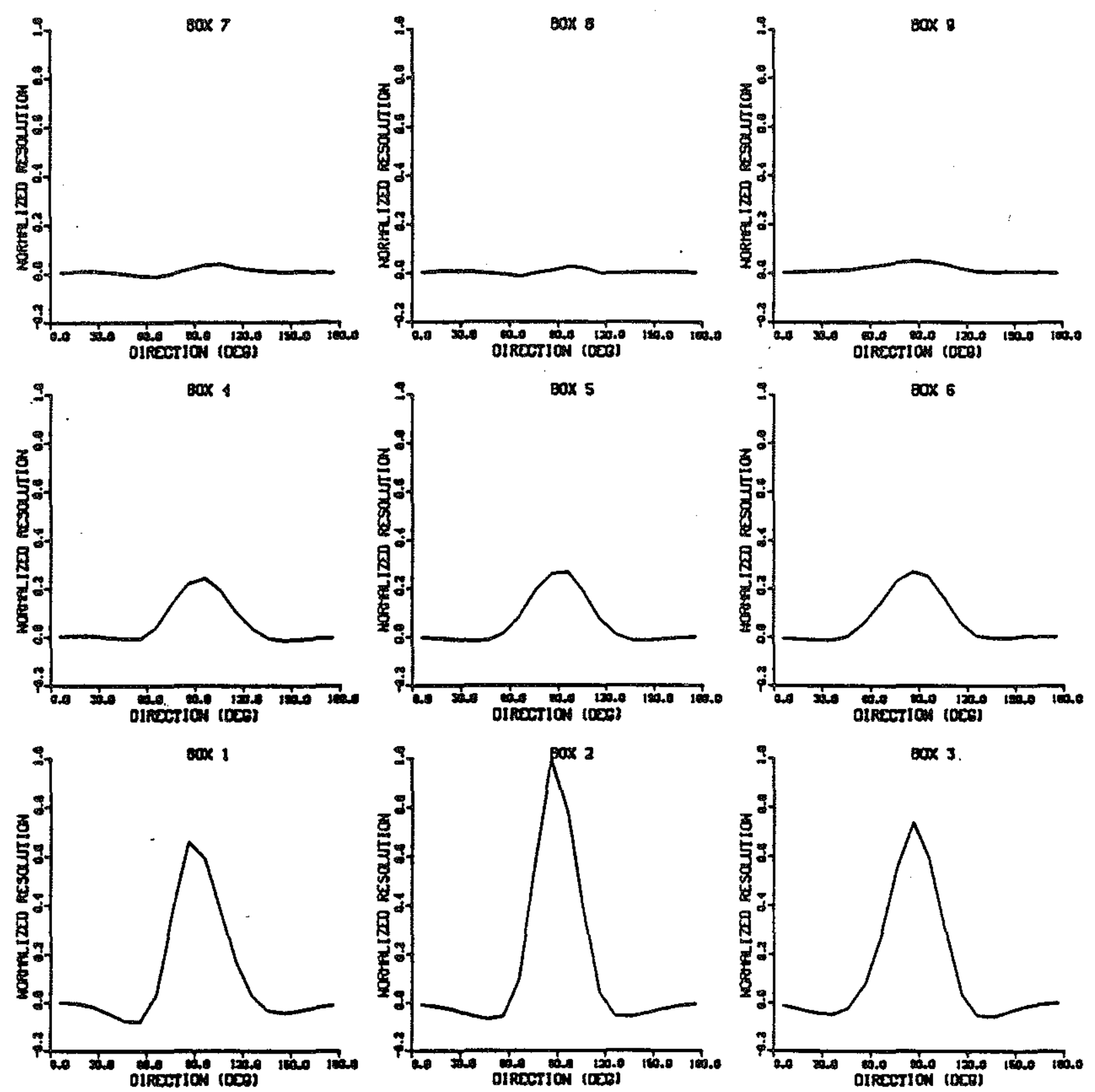

Figure 4.37: Normalized resolution of the frequency-direction spectrum in box 2 at $90 \mathrm{deg}$. 

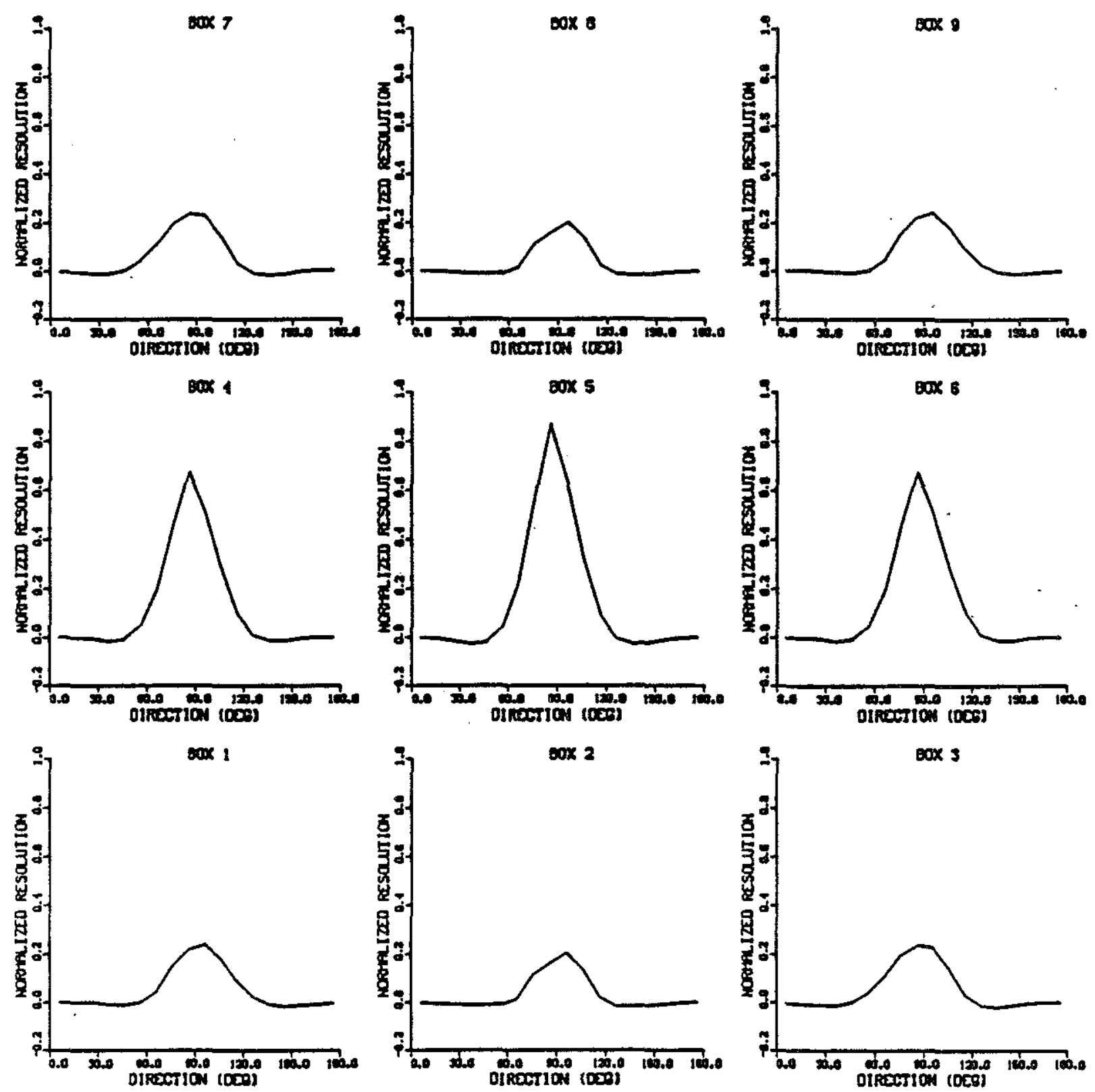

Figure 4.38: Normalized resolution of the frequency-direction spectrum in box 5 at 90 deg. 


\section{Chapter 5}

\section{Conclusions}

\subsection{Summary}

This thesis has developed a new method for estimating quasi-homogeneous, quasi-stationary surface wave frequency-direction spectra using acoustic tomography. The basis of the method is the tomographic inversion of travel time fluctuation spectra of acoustic signals transmitted from a number of sources and measured at a number of receivers.

We showed in Chapter 2 how the phases and travel times of acoustic modes and rays are affected by the height of the sea surface. Acoustic mode eigenvalues can be expressed as a linear function of sea height. Since mode phase, under the WKB approximation, is the integral of the eigenvalue, we showed that the mode phase and travel time are functions of the integral of sea surface height between source and receiver. We showed that the spreading of an acoustic pulse was due to mode coupling and quantified the effect. Adiabatic mode theory and the WKB approximation were shown to generally be adequate for representing the effects of the rough sea on the modes for realistic frequencies, waveguides, and ranges used in tomography. Acoustic ray phase and travel time fluctuations were related to the summed 
sea heights at the ray bounce locations. We derived the relationship between the sea surface time-space covariance and the covariance of the phase and travel time fluctuations for both modes and rays measured at a number of transceivers.

In Chapter 3, we showed that examining the temporal Fourier transform of the sea surface covariance led to the idea that both ray and mode phase/travel time spectra were dependent on the waves which traveled perpendicular to the source/receiver heading, with a 180 degree ambiguity. We showed the application of the theory to data measured in the MIZEX '84 Preliminary Tomography Experiment in the Greenland Sea. In that experiment, only one source and one receiver were deployed so that we could not resolve directional spectra. But the shape of the frequency spectrum and the daily dependence on wind speed strongly suggested that the method is valid.

In Chapter 4, we showed how to estimate the quasi-homogeneous frequency spectrum from the tomographic data. We used a variant of the damped least squares inverse techinque in which the damping by Lagrange multipliers determine the where the solution lies on the resolution/variance tradeoff diagram. The multipliers were varied until reasonable results were obtained. Because we used a known model to generate synthetic data, we were able to calculate the total squared error of our estimate. Variations of the Lagrange multipliers by an order of magnitude one way or the other did not significantly change the total error of the estimate.

We solved a synthetic inverse problem for a fetch-dependent spectrum as a function of location, direction, and frequency. Our inverse technique for the spectra needed only one inverse operation (of size equal to number of directions times number of spatial boxes) independent of frequency and then applied the generalized inverse to the acoustic spectra to estimate the frequency-direction spectra at any frequency desired. The effects of error in the travel time estimation are shown to degrade the frequency-direction spectra estimate at high surface wave frequencies. 
A theoretical variance estimate from linear systems theory is shown to explain these errors. The resolution of the generalized inverse was investigated and used to explain the leakage of energy between spatial boxes.

\subsection{Comparison with Other Methods}

Surface wave tomography has the potential to equal or better the surface wave frequency-direction measurement systems used currently. We have shown that surface wave tomography suffers from a 180 degree ambiguity like some radar methods which also integrate along one spatial dimension, in particular the FM ROWS system.[32] Other radar methods which image the surface directly, such as the Surface Contour Radar (SCR), can eliminate the 180 degree ambiguity.[33] There is a possibility of resolving the ambiguity in surface wave tomography by using the Doppler information of the waves in the direction parallel to the acoustic path, but this is as yet unexplored.

The resolution, in direction, of surface wave tomography depends on the the ratio of acoustic path length and surface wavelength, with typical resolution less than 1 degree. To compare, the SCR has resolution on the order of 10 degrees. However, to sample in direction every 12.5 degrees, we showed that surface wave tomography needed 8 transceivers. The resolution of the pitch and roll buoys is usually not adequate for measuring the angular spread of the spectra.[72] Wave staff arrays have demonstrated directional resolution of 15 degrees.[27]

The spatial resolution of surface wave tomography described in this thesis was $20 \mathrm{~km}$. However, this number could be reduced to $6 \mathrm{~km}$ by changing the spatial box geometry from $320 \times 20 \mathrm{~km}$ boxes to $106 \mathrm{~km}$ strips. The SCR has demonstrated a spatial resolution of $5 \mathrm{~km}$.[33] The frequency resolution of surface wave tomography is determined by the resolution of the spectral estimation method applied to the 
travel time fluctuations.

One limitation of surface wave tomography is its dependence on a linear dispersion relation. It cannot directly measure the wavenumber-frequency spectrum like wave staff arrays can.

\subsection{Original Contributions}

This thesis fulfills one of the requirements for a doctorate from the MIT/WHOI Joint Program in Oceanographic Engineering. As such, it documents an original contribution to the field. However, it is sometimes difficult to separate the original contributions of a thesis between student and advisor. My advisor, Dr. James F. Lynch, contributed some original material to this thesis and in the those sections where I was the original contributor, he was the inspiration and guide. Here, I want to list those sections which are my own original contributions, which were my advisor's, and which are tutorial in nature. Chapter 1 is introductory and historical material.

In Chapter 2, Sections 2.1.1 on range independent normal mode theory, 2.1.2 on range dependent mode theory, and 2.2 .1 on ray eikonal and transport equations, are tutorial in nature. Section 2.1 .6 on mode coupling effects is contributed by Dr. Lynch. Section 2.1.3 on phase and travel time perturbations due to a rough sea surface was essentially a joint effort between Dr. Lynch and myself. Sections 2.1.4 on scattering in canonical waveguides, 2.1.5 on the interpretation of Polcari's mode coherence in the Arctic, 2.1.7 on coupled mode Bragg scattering, 2.1.9 on the application of Desaubies WKB approximation breakdown to mode coupling by the surface, and 2.2.2 on the ray phase and travel time covariance due to a rough surface, are my own original contributions.

In Chapter 3, Sections 3.1 was a review of surface wave spectra. 3.2 on the frequency-direction spectrum, Sections 3.3 on mode phase and travel time spectra, 
3.4 on ray phase and travel time spectra, and 3.6 on the effects of the frozen approximation, are my own original contributions. Section 3.5 is taken predominately from a paper to be published by Lynch, et al.[56] of which I was a co-author. Chapter 4 is mainly my own contribution except for that material explicitly referenced.

\subsection{Significance of the Thesis}

The most significant result of this thesis is the development of a new experimental technique to study sea surface waves, i.e. surface wave tomography. If such an experiment is appropriately designed, estimates of quasi-homogeneous, quasistationary surface wave frequency-directional spectra can be made in addition to the other oceanographic measurements. We feel that an important contribution can be made to the understanding of surface wave/ internal wave interactions by an acoustic tomography experiment using the results of this thesis.

Another significant result is the analysis of mode travel time and phase fluctuations due to surface waves over an acoustic waveguide. In particular, the series expansion of the mode eigenvalue in terms of surface height is quite useful in modelling the effect of sea surface height changes on modal propagation. The first term in the series was shown to represent a linear dependence of surface height and eigenvalue, the basis for surface wave tomography using modes. The second term in the series was shown to be a bias term, i.e., the expected value of the arrival time of the modes was different than that for the mode traveling under a smooth surface. This may be a significant effect in shallow water.

While this thesis developed no new linear inverse theory, the frequency-independent generalized inverse would seem to be unique and useful. Also, the effect of travel time estimation error being magnified at high surface wave frequencies is interesting. 


\subsection{Future Directions}

The natural extension of this thesis is to verify and implement surface wave tomography with an experiment. The simultaneous measurement of surface wave frequency-direction spectra and internal wave directional spectra might contribute to the understanding of the interaction between them. We plan to locate this experiment in the relatively shallow continental shelf region. There, internal waves may have significant directionality, as opposed to the isotropic internal waves in the deeper ocean. This directionality, or equivalently, high correlation between sound speed perturbations measured far apart, would have similiar effects on the acoustic travel times as we have described in this thesis. The acoustic travel time spectra would be most sensitive to internal waves travelling perpendicular to the source/receiver heading, yielding estimates of the internal wave directional spectrum. The shallow depths would enable the acoustic equipment to be moored fixed at the ocean bottom, eliminating the need for position estimation transponders, and may enable less expensive pressure casings to be used. 


\section{References}

[1] W. Munk and C. Wunsch. Ocean acoustic tomography: a scheme for large scale monitoring. Deep-Sea Research, 26A:123-161, 1979.

[2] B. Cornuelle. Acoustic tomography. IEEE Trans. Geosci. Remote Sensing, GE-20(3):326-332, 1982.

[3] The Ocean Tomography Group. A demonstration of ocean acoustic tomography. Nature, 299(5879):121-125, 1982.

[4] Robert C. Spindel. Signal processing in ocean tomography. Reidel, 1985. in Adaptive Methods in Underwater Acoustics.

[5] B. Cornuelle, C. Wunsch, D. Behringer, T. Birdsall, M. Brown, R. Heinmiller, R. Knox, K. Metzger, W. Munk, J. Spiesberger, R. Spindel, D. Webb, and P. Worcester. Tomographic maps of the ocean mesoscale. Part I: Pure acoustics. J. Phys. Oceanogr., 15(2), 1985.

[6] C.-S. Chiu, J. F. Lynch, and O. M. Johannessen. Tomographic resolution of mesoscale eddies in the Marginal Ice Zone - A preliminary study. J. Geophys. Res., 92(C7):6886-6902, 1987.

[7] H. A. DeFerrari and H. B. Nguyen. Acoustic reciprocal transmission experiment, Florida Straits. J. Acoust. Soc. Am., 79(2):299-315, 1986.

[8] S. M. Flatte and R. B. Stoughton. Theory of acoustic measurements of internal wave strength as a function of depth, horizontal position, and time. J. Geophys. Res., 91(C6):1709-1732, 1986.

[9] P. J. Bushong. Tomographic Measurements of Barotopic Motions. Master's thesis, MIT/WHOI Joint Program, Cambridge and Woods Hole, September 
1987.

[10] G. Neumann and W. J. Pierson. Known and unknown properties of the frequency spectrum of a wind-generated sea. In Ocean Wave Spectra, pages 9-25, Prentice-Hall, Englewood Cliffs, 1963.

[11] C. C. Tung and N. E. Huang. Covariances and spectra of the kinematics and dynamics of nonlinear waves. J. Geophys. Res., 90(C6):11911-11916, 1985.

[12] M. S. Longuet-Higgins. On the statistical distribution of the heights of sea waves. J. Mar. Res., 11:245-266, 1952.

[13] M. S. Longuet-Higgins. Resonant interactions between two trains of gravity waves. J. Fluid Mech., 12:321-322, 1962.

[14] W. J. Pierson, G. Neumann, and R. W. James. Practical methods for observing and forecasting ocean waves by means of wave spectra and statistics. Technical Report 603, U.S. Navy Hydrographic Office, 1955.

[15] K. Hasselmann. On the non-linear energy transfer in a gravity wave spectrum. J. Fluid Mech., 12:481-500, 1962.

[16] A. Papoulis. Probability, Random Variables, and Stochastic Processes. McGraw-Hill, New York, 1965.

[17] T. P. Barnett and K. E. Kenyon. Recent advances in the study of wind waves. Rep. Prog. Phys., (38):667-729, 1975.

[18] O. M. Phillips. The Dynamics of the Upper Ocean. Cambridge University Press, New York, 1966. 
[19] L. F. McGoldrick. A sensitive linear capacitance-to-voltage converter, with applications to surface wave measurements. Rev. Sci. Instrum., (42):359-361, 1971.

[20] F. E. Snodgrass, G. W. Groves, K. F. Hasselmann, G. R. Miller, W. F. Munk, and W. H. Powers. Propagation of ocean swell across the Pacific. Phil. Trans. R. Soc. Lond., (A259):431-497, 1966.

[21] L. J. Cote, J. O. Davis, W. Marks, R. J. McGough, E. Mehr, W. J. Pierson, J. F. Ropek, G. Stephenson, and R. C. Vetter. The directional spectrum of wind-generated sea as determined from data obtained by the stereo wave observation project. Technical Report, Meteor. Pap., New York University, School of Engineering, 1960. vol. 2, no. 6, (88 pages).

[22] D. E. Cartwright and N. D. Smith. Buoy techniques for obtaining directional wave spectra. In Buoy Technology, pages 112-121, Marine Technology Society, Washington, D.C., 1964.

[23] H. Mitsuyasu, F. Tasai, T. Suhara, S. Mizuno, M. Ohkuso, T. Honda, and K. Rikiishi. Observations of the directional spectrum of ocean waves using a cloverleaf buoy. J. Phys. Oceanogr., 5:750-760, 1975.

[24] D. E. Hasselmann, M. Dunckel, and J. A. Ewing. Directional spectra observed during JONSWAP 1973. J. Phys. Oceanogr., 10:569-573, 1980.

[25] N. F. Barber. The directional resolving power of an array of wave recorders. In Ocean Wave Spectra, pages 137-150, Prentice-Hall, Englewood Cliffs, 1963.

[26] W. M. Munk, F. E. Snodgrass, and N. F. Barber. Directional recording of swell from distant storms. Phil. Trans. R. Soc. Lond., A255:505-584, 1963. 
[27] M. A. Donelan, J. Hamilton, and W. H. Hui. The directional spectra of windgenerated waves. Phil. Trans. R. Soc. Lond., A-315:509-582, 1985.

[28] S. H. Hill. Acoustic remote sensing of waveheight directional spectra of surface gravity waves. In Proc. 15th Symp. Acoust. Imag. and 12 ICA Assoc. Symp. Under. Acoust., Plenum, July 1986.

[29] L. C. Huff and R. G. Williams. Acoustic remote sensing of waveheight. Eos Trans. $A G U, 67(44): 1010,1986$. Abstract from AGU Fall Meeting.

[30] J. A. Smith and R. Pinkel. Surface wave measurements by Doppler sonar. Eos Trans. AGU, 67(44):1060, 1986. Abstract from AGU Fall Meeting.

[31] F. M. Labianca. Estimation of ocean-surface directional-frequency spectra: The inverse scattering problem. J. Acoust. Soc. Am., 67(5):1567-1577, 1980.

[32] W. J. Plant. The microwave measurement of ocean-wave directional spectra. In Measuring Ocean Waves from Space, pages 55-59, The Johns Hopkins University Applied Physics Laboratory, April 1986.

[33] E. J. Walsh, D. W. Hancock III, D. E. Hines, R. N. Swift, and J. F. Scott. Directional wave spectra measured with the Surface Contour Radar. J. Phys. Oceanogr., 15:566-592, 1985.

[34] R. C. Beal, T.W. Gerling, D.E. Irvine, F.M. Monaldo, and D. G. Tilley. Spatial variations of ocean wave directional spectra from the SEASAT synthetic aperture radar. J. Geophys. Res., 91(C2):2433-2449, 1986.

[35] D. R. Lyzenga. The physical basis for estimating wave energy spectra from SAR imagery. In Measuring Ocean Waves from Space, pages 65-69, The Johns Hopkins University Applied Physics Laboratory, April 1986. 
[36] F. M. Monaldo. A practical methodology for estimating waves spectra from the SIR-B. In Measuring Ocean Waves from Space, pages 82-85, The Johns Hopkins University Applied Physics Laboratory, April 1986.

[37] K. M. Watson, B. J. West, and B. I. Cohen. Coupling of surface and internal gravity waves: a mode coupling model. J. Fluid Mech., 77(1):185-208, 1976.

[38] D. J. Olbers and K. Herterich. The spectral energy transfer from surface waves to internal waves. J. Fluid Mech., 92(2):349-379, 1979.

[39] S. R. Rutherford. An Examination of Coupled Mode Theory as Applied to Underwater Sound Propagation. Technical Report 79-44, Appl. Res. Lab., University of Texas at Austin, 1979.

[40] L. M. Brekovskikh. Waves in Layered Media. Academic Press, New York, 1960.

[41] C. S. Clay and H. Medwin. Acoustical Oceanography. Wiley, New York, 1977.

[42] A. D. Pierce. Extension of the method of normal modes to sound propagation in an almost-stratified medium. J. Acoust. Soc. Am., 37:19-27, 1965.

[43] D. M. Milder. Ray and wave invariants for sofar channel propagation. $J$. Acoust. Soc. Am., 46:1259-1263, 1969.

[44] C. T. Tindle and K. M. Guthrie. Ray effects in the normal mode approach to underwater acoustics. J. Sound Vib., 34(2):291-295, 1974.

[45] M. Abramowitz and I. A. Stegun. Handbook of Mathematical Functions. Dover Publications, New York, ninth edition, 1970.

[46] J. Polcari. Acoustic Mode Coherence in the Arctic Ocean. PhD thesis, MIT/WHOI Joint Program, Cambridge and Woods Hole, April 1986. 
[47] J. F. Lynch, J. H. Miller, C. S. Chiu, and G. V. Frisk. Multiple scattering of normal modes from rough surfaces with applications to shallow water acoustics,' Arctic acoustics, and ocean acoustic tomography. J. Acoust. Soc. Am., 1987. submitted for publication.

[48] J. R. Taylor. Scattering Theory. Wiley, New York, 1972.

[49] S. T. McDaniel. Mode conversion in shallow water sound propagation. $J$. Acoust. Soc. Am., 62(2):320-325, 1977.

[50] S. T. McDaniel. Mode coupling due to interaction with the seabed. J. Acoust. Soc. Am., 73(3):916-923, 1982.

[51] F. Press. Handbook of Physical Constants - revised edition. Geol. Soc. Am. Mem., 97:207, 1966.

[52] Y. J. F. Desaubies. On the scattering of sound by internal waves in the ocean. J. Acoust. Soc. Am., 54:1460-1469, 1978.

[53] Y. Desaubies, C. S. Chiu, and J. H. Miller. Acoustic mode propagation in a range dependent ocean. J. Acoust. Soc. Am., 80(4):1148-1160, 1986.

[54] L. M. Brekovskikh and Y. Lysanov. Fundamentals of Ocean Acoustics. Springer-Verlag, New York, 1982.

[55] R. Michael Jones, J. P. Riley, and T. M. Georges. HARPO, A Versatile Three-Dimensional Hamiltonian Ray-Tracing Program for Acoustic Waves in an Ocean with Irregular Bottom. Technical Report, Wave Propagation Laboratory, NOAA, U. S. Dept. of Commerce, Boulder, Colorado, October 1986.

[56] J. F. Lynch, R. C. Spindel, C. S. Chiu, J. H. Miller, and T. G. Birdsall. Results from the MIZEX '84 preliminary tomography experiment: implications for the 
MIZ, Arctic, and surface wave tomography. J. Geophys. Res., 92(C7):6869$6885,1987$.

[57] I. Tolstoy. Wave Propagation. McGraw-Hill, 1973.

[58] J. S. Bendat and A. G. Piersol. Random Data: Analysis and Measurement Procedures. Wiley-Interscience, New York; 1971.

[59] A. V. Oppenheim and R. W. Schafer. Digital Signal Processing. Prentice Hall, Inc., Englewood Cliffs, New Jersey, 1975.

[60] I. S. Gradshteyn and I. M. Ryzhik. Table of Integrals, Series, and Products. Academic Press, New York, corrected and enlarged edition, 1980. page 446.

[61] G. L. Pickard and W. J. Emery. Descriptive Physical Oceanography. Pergamon, New York, 1983.

[62] E. Sundvor, A. M. Myhre, A. Austegard, K. Hauglaud, O. Eldholm, and A. Gidskehang. Marine geophysical survey on the Yermak Plateau. Technical Report 7, U. Bergen Seismol. Observ. Sci. Rep., Bergen, 1982.

[63] K. Hasselmann, D. B. Ross, P. Muller, and W. Sell. A parametric wave prediction model. J. Phys. Oceanogr., 6(2):200-228, 1976.

[64] P. B. Liebelt. An Introduction to Optimal Estimation. Addison-Wesley, Reading, 1967.

[65] J. N. Franklin. Well-posed stochastic extensions of ill-posed linear problems. J. Math. Anal. Appl., 31:682-716, 1970.

[66] D. D. Jackson. Interpretation of inaccurate, insufficient and inconsistent data. Geophys. J. R. Astr. Soc., 28:97-109, 1972. 
[67] T. H. Jordan and J. N. Franklin. Optimal solutions to a linear inverse problem in geophysics. Proc. Nat. Acad. Sci., 68:291-293, 1971.

[68] C. Provost. A Variational Method for Estimating the General Circulation in the Ocean. PhD thesis, University of California, San Diego, 1983.

[69] W. Menke. Geophysical Data Analysis: Discrete Inverse Theory. Academic Press, Orlando, 1984.

[70] C. T. Tindle and G. E. J. Bold. Improved ray calculations in shallow water. J. Acoust. Soc. Am., 70(3):813-819, 1981.

[71] C.-S. Chiu and Y. Desaubies. A planetary wave analysis using the acoustic and conventional arrays in the 1981 Ocean Tomography Experiment. J. Phys. Oceanogr., 1987. in press.

[72] W. J. Pierson. Some approaches for comparing remote and in-situ estimates of directional spectra. In Measuring Ocean Waves from Space, pages 48-54, The Johns Hopkins University Applied Physics Laboratory, April 1986. 


\section{Biography}

Since 1983, James H. Miller has been a doctoral student in the Massachusetts Institute of Technology - Woods Hole Oceanographic Institution Joint Program in Oceanographic Engineering. Mr. Miller is affiliated with the Department of Ocean Engineering at MIT and the Department of Ocean Engineering at WHOI. From 1981 to 1983, he was a Member of the Professional Staff at The Analytic Sciences Corporation in Reading, Massachusetts. He received a MS in Electrical Engineering from Stanford University in 1981 and a BS in Electrical Engineering from Worcester Polytechnic Institute in 1979. In the summer of 1979, he was a Member of the Technical Staff at Sanders Associates in Nashua, New Hampshire.

Mr. Miller is a member of Sigma Xi, Tau Beta Pi, Eta Kappa Nu, the Institute of Electrical and Electronics Engineers (IEEE), the Acoustical Society of America (ASA), and the American Geophysical Union (AGU). 